\title{
A Calorimetric Measurement of the Strong Coupling Constant in Electron-Positron Annihilation at a Center-of-Mass Energy of \\ 91.6 GeV
}

by

Saúl González Martirena

B.S., Georgia Institute of Technology, (1986)

Submitted to the Department of Physics

in partial fulfillment of the requirements for the degree of

$$
\begin{aligned}
& \text { Doctor of Philosophy } \\
& \text { at the }
\end{aligned}
$$

\section{MASSACHUSETTS INSTITUTE OF TECHNOLOGY}

$$
\text { May } 1994
$$

(C) Massachusetts Institute of Technology 1994. All rights reserved.

Author

Department of Physics

March 14, 1994

Certified by .

\section{Jerome I. Friedman Institute Professor Thesis Supervisor}

Accepted by

George F. Koster Chairman, Departmental Committee on Theses MAY 251994

IBRARIES

Science 



\title{
A Calorimetric Measurement of the Strong Coupling Constant in Electron-Positron Annihilation at a Center-of-Mass Energy of $91.6 \mathrm{GeV}$
}

by Saúl González Martirena

Submitted to the Department of Physics on March 14, 1994, in partial fulfillment of the requirements for the degree of

Doctor of Philosophy

\begin{abstract}
In this work, a measurement of the strong coupling constant $\alpha_{s}$ in $e^{+} e^{-}$annihilation at a center-of-mass energy of $91.6 \mathrm{GeV}$ is presented. The measurement was performed with the SLD at the Stanford Linear Collider facility located at the Stanford Linear Accelerator Center in California.

The procedure used consisted of measuring the rate of hard gluon radiation from the primary quarks in a sample of 9,878 hadronic events. After defining the asymptotic manifestation of partons as 'jets', various phenomenological models were used to correct for the hadronization process. A value for the QCD scale parameter $\Lambda_{\overline{M S}}$, defined in the $\overline{M S}$ renormalization convention with 5 active quark flavors, was then obtained by a direct fit to $\mathcal{O}\left(\alpha_{s}^{2}\right)$ calculations. The value of $\alpha_{s}$ obtained was $\alpha_{s}\left(M_{Z^{0}}\right)=0.122 \pm 0.004+0.008$ where the uncertainties are experimental (combined statistical and sytematic) and theoretical (systematic) respectively. Equivalently, $\Lambda_{\overline{M S}}=0.28_{-0.10}^{0.16} \mathrm{GeV}$ where the experimental and theoretical uncertainties have been combined.
\end{abstract}

Thesis Supervisor: Jerome I. Friedman

Title: Institute Professor 



\section{Acknowledgments}

I have had the privilege of learning from the best. My advisor, Jerry Friedman, has been an indescribable source of encouragement and support throughout my years in graduate school. I am very grateful for his guidance and for his heroic efforts on behalf of Science. His friendship will always be a source of strength and direction for me.

I have been very fortunate to be a member of the Counter Spark Chamber group, a great bunch of physicists and friends. I will miss and always remember the lunch hours in room 24-515 and the many eclectic conversation topics.

Wit Busza has been a good teacher. His advice and leadership, especially during the SLD years, were essential to my education. Larry Rosenson has always been willing to help with any problem; his help and advice have been essential for the completion of this thesis. I wish to thank Henry Kendall for his advice and for awakening in me an awareness of the humanistic side of physics. His support and encouragement have meant a great deal to me. I also wish to thank Henry for the opportunity to help him in the freshman lab. Through the years I have benefitted from many conversations, not just about physics, with Louis Osborne. Frank Taylor has always been very supportive and eager to listen and help with any problem. I have learned from Frank that good physics leadership means staying intimately in touch with the physics. Robin Verdier has been a great source of everything. He has taught me a lot about physics, statistics, computers, and jazz. His help was essential in my transition from SLAC back to MIT.

During my last year at MIT, I had the opportunity to cross paths with many new faces. I wish to thank Leslie Rosenberg and Bolek Wyslouch for their friendship and their support. Leslie helped me keep my sense of humor alive and Bolek helped me keep my Spanish alive. Another nice thing about coming back to MIT was getting to know John Ryan and Mark Baker. John and Mark were always willing to help me with anything. With their help, I have saved many months of work and gained a fine appreciation for Guiness Stout. Ed Daw has been a great friend that frequently rescued me from the office to "go to the Pub". I have enjoyed and benefitted from the many conversations with Tushar Shah and Ken Halpern.

I made many friends during my years at SLAC. John Yamartino has been an officemate, a roommate, and a physicsmate. Andrea Higashi stole my roommate and my apartment but she paid me back with her friendship. Dave Williams was always willing to listen to my computer problems and immediately solve them. Suzanne Williams provided countless cookies that kept all of us going. Amit Lath has been a great friend who was always willing to help and to listen. I have shared many beers with Philip Burrows while at SLAC. I will always remember our many trips to Point Reyes and my unexpected dip into the mighty Pacific. I also wish to thank Richard Dubois, Kevin Pitts, Hwanbae Park, Ram Ben-David, and Sarah Hedges. I learned a great deal of hardware during my early years at SLAC from the Italian contingent, especially from Livio Piemontese and Alberto Benvenuti. In addition, I wish to thank all of the people in SLD that gave me the opportunity to work with them. I wish them the best of success in the coming years. 
I want to thank Phillip Burrows, Henry Kendall, and Ken Halpern for reading sections of the manuscript and offering valuable suggestions. I wish to thank Peggy Berkovitz for her help with my academic and administrative matters. I also wish to thank Sandy Fowler for her invaluable help while at SLAC and after my return to MIT. I am also very grateful to Margaret Helton at SLAC.

I wish to thank the MIT Graduate School for providing me with a Fellowship during my early years at MIT and the U. S. Department of Energy for subsequent support.

Finally, I wish to thank my family in Puerto Rico and in Atlanta. Their love and total support have been more than essential for me. My sister, my nephews, and my brother-in-law have been a great source of joy and support. I have no words to express how grateful I am to my parents. It is to them that I dedicate this effort. I thank them for having had the courage and the vision many years ago to leave behind what was dear and familiar to them in order to seek a better future for my sister and myself. Mom and Dad: it paid off. This PhD is all yours. 


\section{Contents}

1 Intraduction 15

1.1 Motivation . . . . . . . . . . . . . . . . . 15

1.2 Thesis Outline . . . . . . . . . . . . . . . . 17

1.3 Electroweak Interactions $\ldots \ldots \ldots \ldots \ldots$

2 Quantum Chromodynamics 21

2.1 Development of QCD . . . . . . . . . . . . . . . . . . 21

2.2 The Theory . . . . . . . . . . . . . . . . . . . . 22

2.2 .1 Renormalization. . . . . . . . . . . . . . 24

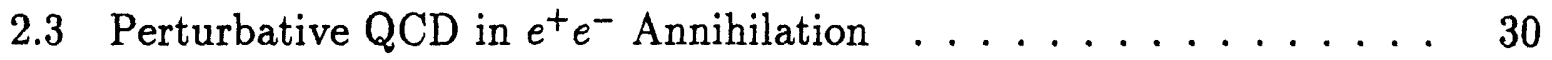

2.3.1 Experimental Developments . . . . . . . . . . . . 30

2.3.2 QCD Perturbative Predictions . . . . . . . . . . . . 31

2.4 Non-Perturbative QCD . . . . . . . . . . . . . . . . . . . . 37

2.4 Pre-fragmentation . . . . . . . . . . . . . . 37

2.4 .2 Hadronization . . . . . . . . . . . . . . . . . 39

3 Experimental Apparatus 43

3.1 Introduction . . . . . . . . . . . . . . . . . . 43

3.2 The Stanford Linear Collider . . . . . . . . . . . . . . . . . . . . 43

3.2 .1 The Polarized SLC . . . . . . . . . . . . . . . . . 43

3.2.2 Beam Energy Measurement . . . . . . . . . . . . . . . 46

3.3 The Stanford Large Detector . . . . . . . . . . . . . . . . . . . 46

3.3.1 The Vertex Detector . . . . . . . . . . . . . . . . . . . 47

3.3.2 The Luminosity Monitor . . . . . . . . . . . . . . . . 49

3.3.3 The Drift Chamber System . . . . . . . . . . . . . 50

3.3.4 The Cherenkov Ring Imaging Device . . . . . . . . . . 51

3.3.5 The Calorimeter System . . . . . . . . . . . . . . . 51

3.4 The SLD Monte Carlo . . . . . . . . . . . . . . . . . . . 58

3.5 The SLD Event Reconstruction . . . . . . . . . . . . . . . 59

4 Calibration of the SLD Calorimeter 63

4.1 Introduction . . . . . . . . . . . . . . . 63

$4.2 \quad$ Scales . . . . . . . . . . . . . . . . . . . . . 64

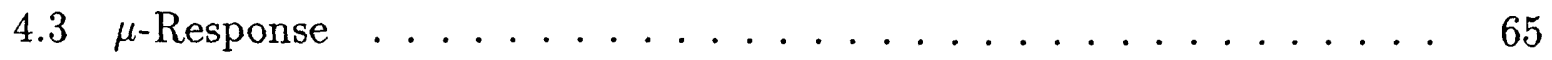

4.3 .1 Cosmic Rays . . . . . . . . . . . . . . . . 65 
4.3.2 $\mu$-Response from single clusters $\ldots \ldots \ldots \ldots$. . . . . 67

4.4 Electromagnetic Response . . . . . . . . . . . . . . . 70

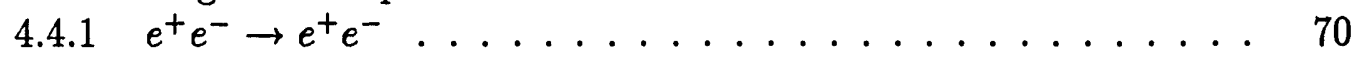

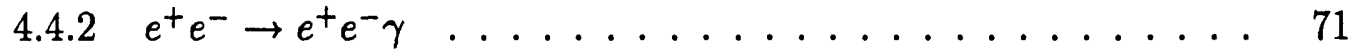

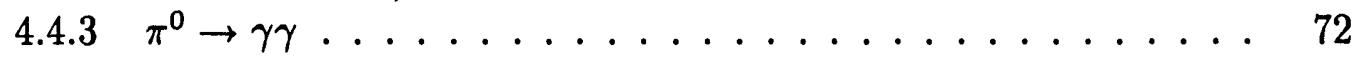

4.5 Hadronic Response . . . . . . . . . . . . . . . . 73

$4.5 .1 \quad$ Energy Flow . . . . . . . . . . . . . . . 75

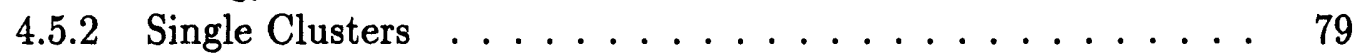

4.6 Tuning of the SLD Monte Carlo . . . . . . . . . . . . . . 82

4.7 A Brief Comment on $\frac{e}{\pi} \ldots \ldots \ldots \ldots \ldots \ldots \ldots$

4.8 Summary . . . . . . . . . . . . . . . 85

5 Triggering and Event Selection $\quad \mathbf{8 7}$

5.1 Properties of the 1992 Run . . . . . . . . . . . . . . . 88

5.1 .1 Luminosity in the SLD . . . . . . . . . . . . . 88

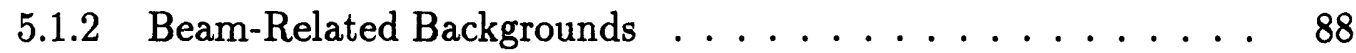

5.2 Physics Backgrounds . . . . . . . . . . . . . . . . 92

5.3 The Hadronic Event Trigger . . . . . . . . . . . . . . . . . 94

5.4 Event Filtering $\ldots \ldots \ldots \ldots \ldots \ldots \ldots \ldots \ldots$

5.5 Hadronic Event Selection . . . . . . . . . . . . . . . . . . . 97

5.6 Results . . . . . . . . . . . . . . . . . . . . . . 102

6 The Measurement 105

6.1 Introduction . . . . . . . . . . . . . . . . . . . . 105

6.2 Jets in the SLD Calorimeter . . . . . . . . . . . . . . . 105

6.2.1 Definition of Jets and Jet Algorithms . . . . . . . . . . . . 109

6.2.2 A Question of Resolution and Bias . . . . . . . . . . . 113

6.3 Corrections to the Jet-Fractions . . . . . . . . . . . . . . . 117

6.3.1 Initial State Radiation . . . . . . . . . . . . . . . . . 118

6.3 .2 Hadronization Effects . . . . . . . . . . . . . . . 118

6.3 .3 Particle Decays . . . . . . . . . . . . . . . . . . . . . 121

6.3.4 Detector Effects . . . . . . . . . . . . . . . . . . . . 121

6.3.5 Putting It All Together . . . . . . . . . . . . . . . . . . 122

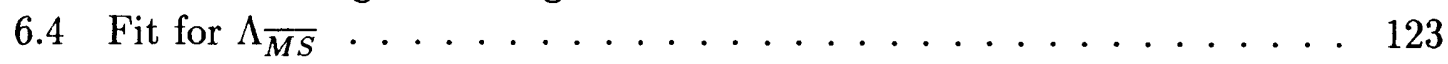

6.5 Systematic Effects . . . . . . . . . . . . . . . . . . . . . 129

6.5.1 Experimental Systematics . . . . . . . . . . . . . 130

6.5 .2 Theoretical Systematics . . . . . . . . . . . . . 132

6.6 Results . . . . . . . . . . . . . . . . . 136

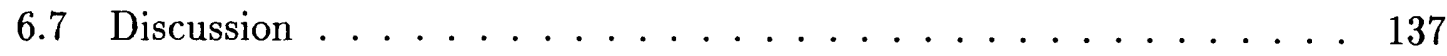

6.7 .1 Running of $\alpha_{s} \ldots \ldots \ldots \ldots \ldots \ldots \ldots$

6.7.2 Other Measurements of $\alpha_{s}$ and Final Thoughts . . . . . 141

$\begin{array}{ll}\text { A Observables } & 145\end{array}$

A.1 Introduction . . . . . . . . . . . . . . . . 145

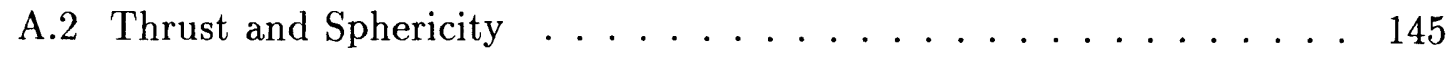




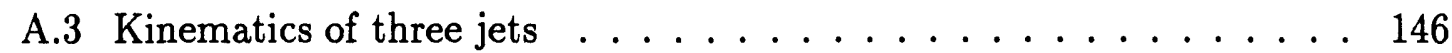

A.4 Differential Jet Rates . . . . . . . . . . . . . . . . 147

B Detector Correction Procedure $\quad 149$

B.1 Introduction . . . . . . . . . . . . . . . . . . 149

B.2 The Inversion Method . . . . . . . . . . . . . . . . . 149

B.3 The Factor Method . . . . . . . . . . . . . . . . . 152

B.4 Validating the Factor Method . . . . . . . . . . 153 


\section{List of Figures}

1-1 Tree Level Feynman diagrams for $q \bar{q}$ Production . . . . . . . . . . . 18

1-2 Cross section for $e^{+} e^{-} \rightarrow \mu^{+} \mu^{-} \ldots \ldots \ldots \ldots \ldots$

2-1 Some QCD Vertex Factors . . . . . . . . . . . . . . . . . . . 24

2-2 Leading Order Corrections . . . . . . . . . . . . . . . . . . . 29

2-3 $\mathcal{O}\left(\alpha_{s}\right)$ diagrams for parton production. . . . . . . . . . . 33

2-4 Phase Space Region for 3-Parton Final States . . . . . . . . . 33

$2-5\left\langle N_{\text {parton }}\right\rangle$ vs $Q_{0} \ldots \ldots \ldots \ldots \ldots \ldots \ldots \ldots \ldots \ldots$

2-6 Schematic of Shower and Matrix Element Models . . . . . . . . . . . 39

3-1 The Polarized SLC . . . . . . . . . . . . . . . . . . . . . . . 44

3-2 The Energy Spectrometer . . . . . . . . . . . . . . . . . 47

3-3 A quadrant view of SLD . . . . . . . . . . . . . . . . 48

$3-4$ View of the SLD Vertex Detector . . . . . . . . . . . . . . 49

3-5 Side view of LMSAT/MASiC Assembly . . . . . . . . . . . . 50

3-6 Exploded View of the LAC Barrel . . . . . . . . . . . . . . 52

3-7 A LAC Barrel Module . . . . . . . . . . . . . . . . . . . . . . 53

3-8 Exploded View of the LAC EndCap . . . . . . . . . . . . . 53

3-9 A LAC EndCap Module . . . . . . . . . . . . . . . . . . . . . 54

3-10 Detail of the LAC Cell Arrangement . . . . . . . . . . . . . . . 54

$3-11 X_{0}$ and $\lambda_{\text {int }}$ as a Function of $\theta \ldots \ldots \ldots \ldots$

3-12 Plot of $G_{c}$ for the Barrel Region . . . . . . . . . . . . . . 61

3-13 Global observables for two cluster selection methods . . . . . . . . . . 61

4-1 Muon Penetration as a Function of $p_{i n c} \ldots \ldots \ldots 66$

4-2 Cosmic ray layer-by-layer mip signals . . . . . . . . . . . . 67

4-3 mip Clusters in Hadronic Events . . . . . . . . . . . . . . . . . . 68

4-4 Endcap mip from Single Clusters. . . . . . . . . . . . . . . . . 69

4-5 Muon Energy as a Function of $\cos \theta \ldots \ldots \ldots \ldots$

$4-6$ Muon Energy as a Function of $\phi \ldots \ldots \ldots \ldots$. . . . . . . 70

4-7 Visible Energy in Bhabha Events . . . . . . . . . . . . . . 71

4-8 Expected Energy for Radiative Bhabhas . . . . . . . . . . . . 72

4-9 Angle Definition for Radiative Bhabhas . . . . . . . . . . . . . . 72

4-10 Plot of the Two-Photon Invariant Mass . . . . . . . . . . . . . 74

4-11 Schematic of the Energy Flow Calibration Regions . . . . . . . . 76

4-12 Energy Flow Calibration for the LAC . . . . . . . . . . . . 77 
4-13 Energy Flow Calibration for the LAC and WIC . . . . . . . . . 77

4-14 Plot of the Hadronic Response as a Function of $p \ldots \ldots$

4-15 Ratio of Energy Response to Incident Momentum . . . . . . . . . . 81

4-16 The Single Cluster Energy Resolution . . . . . . . . . . . . 81

4-17 Energy Distribution of Single Clusters . . . . . . . . . . . 82

4-18 Comparison of Observables in Monte Carlo and Data . . . . . . . . 83

5-1 A Beam Background Event . . . . . . . . . . . . . . 90

5-2 SLC-induced Muons During the 1992 Run . . . . . . . . . . . . . . . 91

5-3 Backgrounds to the Event Selection . . . . . . . . . . . . . 93

5-4 Hit Spectra of Muon Backgrounds . . . . . . . . . . . . . . . 94

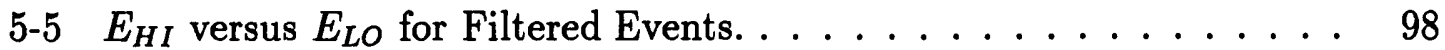

5-6 Plot of $N_{H I}^{e m}$ for Filtered Events. . . . . . . . . . . . . . . . 98

5-7 $E_{H I}$ for Filtered Data and Monte Carlo with Backgrounds . . . . 98

5-8 $E_{L O}$ for Filtered Data and Monte Carlo with Backgrounds . . . . . . 99

$5-9$ Energy Imbalance for Filtered Events. . . . . . . . . . . . . . 100

5-10 Number of Clusters for Filtered Events . . . . . . . . . . . . . . 100

5-11 Visible Energy for Filtered Events . . . . . . . . . . . . . . . 101

5-12 EndCap/Barrel $E_{v i s}$ for Pre-selected Events . . . . . . . . . . . 101

$5-13 E_{v i s}$ vs $\cos \theta_{t h r u s t}$ for Filtered Events . . . . . . . . . . . 103

5-14 A 3-Jet Event . . . . . . . . . . . . . . . 104

6-1 Total Energy Distribution for Tracking and Calorimetry . . . . . . 106

6-2 Some QCD Observables in the SLD Calorimeter . . . . . . . . . . . . 107

6-3 Thrust Distribution for Observed Data. . . . . . . . . . . . . 108

$6-4 y_{3}$ Distribution. . . . . . . . . . . . . . . 108

6-5. Raw Jet-Fractions . . . . . . . . . . . . . . . . 111

6-6 $y_{3}$ detector-hadron correlation. . . . . . . . . . . . . 114

6-7 Detector-hadron resolution for the JADE algorithm. . . . . . . . . . 114

6-8 Algorithm resolution: hadron-parton . . . . . . . . . . . . . 115

6-9 Jet resolution with Different calorimeter layers . . . . . . . . . . . 116

6-10 The Hadronization Smearing Matrix $S(y) \ldots \ldots$. . . . . . . . 119

6-11 Parton/Hadron Jet-Fractions . . . . . . . . . . . . 120

6-12 The Detector Correction Factors and Matrix. . . . . . . . . . . . 122

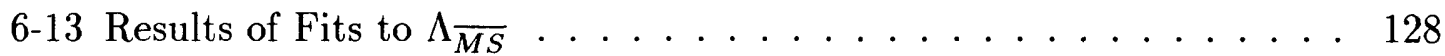

6-14 Average Differential Jet Rates as a Function of Time . . . . . . . . . 131

6-15 Differential Jet Rates for EndCap/Barrel Clusters . . . . . . . . . . 131

6-16 Monte Carlo Test of Algorithm Bias. . . . . . . . . . . . . 133

$6-17 \chi^{2}$ Sensitivity to Missing Terms. . . . . . . . . . . . . . 133

$6-18$ The fixed and optimized scale fits. . . . . . . . . . . . 138

6-19 Running of $R_{3}$ with $E_{c m} \ldots \ldots \ldots \ldots 141$

B-1 The Detector Correction Factors and Matrix. . . . . . . . . . . 151

B-2 $\Lambda_{\overline{M S}}$ (thrown) vs $\Lambda_{\overline{M S}}$ (measured) for Test Experiment $\ldots \ldots \ldots 154$ 


\section{List of Tables}

1.1 Electroweak Quantum Numbers . . . . . . . . . . . . 18

2.1 LUND fragmentation Parameters . . . . . . . . . . . 41

3.1 LAC Longitudinal Segmentation . . . . . . . . . . . . . 56

4.1 Summary of Minimum Ionizing Fits . . . . . . . . . . . . . 68

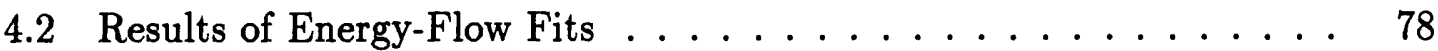

4.3 Longitudinal Energy Fractions . . . . . . . . . . . . . . 78

4.4 Summary of Energy Scales . . . . . . . . . . . . . . . 85

5.1 Summary of Trigger Quantities $\ldots \ldots \ldots \ldots \ldots$

5.2 Event Selection Yields . . . . . . . . . . . . . . . . . 103

5.3 Summary of Backgrounds . . . . . . . . . . . . . . . . . . 104

6.1 Multi-jet Cross Section: JADE Algorithm . . . . . . . . . . 124

6.2 Multi-jet Cross Section: P-scheme . . . . . . . . . . . . . . . . 124

6.3 Multi-jet Cross Section: E-scheme . . . . . . . . . . . . . . 125

6.4 Multi-jet Cross Section: Durham . . . . . . . . . . . . 125

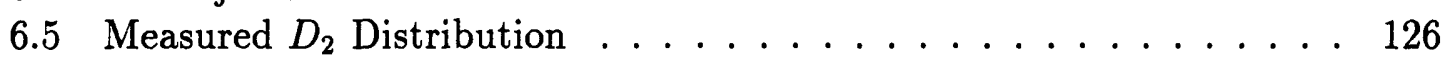

6.6 Summary of Fit Results: $\Lambda_{\overline{M S}} \ldots \ldots \ldots \ldots \ldots \ldots \ldots$

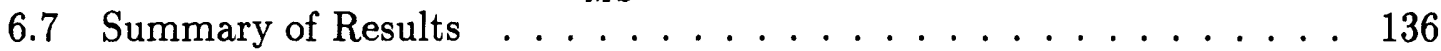

6.8 Fit Results for $\mu^{2}=y_{3} E_{c m}^{2} \quad \ldots \ldots \ldots \ldots \ldots \ldots \ldots \ldots$

6.9 Other Experiments . . . . . . . . . . . . . . . . . . 142 


\section{Chapter 1}

\section{Introduction}

\subsection{Motivation}

The testing of Quantum Chromodynamics, the theory of strong interactions, at the perturbative scale is by no means a closed book. The nature of the strong force is such that perturbative calculations are only reliable in the high energy limit. This limit, however, does not include hadronization, the QCD mechanism for particle production. One then resorts to phenomenological models in order to complete the picture. This fact, and the fact that the large coupling makes any calculation very sensitive to a truncation in the perturbative series, introduces sizeable uncertainties in any measurement. The understanding of these uncertainties then becomes the central issue in any perturbative QCD (PQCD) measurement.

With these issues in mind, we proceeded to determine the QCD strong coupling constant in $e^{+} e^{-}$annihilation. In this chapter we present the motivation for this measurement, a short description of the Standard Model of Electroweak Interactions, and a brief outline of the rest of the thesis.

\section{Theoretical Motivation}

There is no doubt that the most important QCD measurement in the perturbative regime $\left(Q^{2} \gg \Lambda_{Q C D}^{2}\right)$ is the measurement of the strong coupling $\alpha_{s}\left(Q^{2}\right)$. Paradoxically this measurement, on its own, does not tell us much about QCD or anything else. The importance of this measurement, as far as QCD is concerned, lies solely in a demonstration of the expected running of $\alpha_{s}\left(Q^{2}\right)$ with the interaction scale $Q^{2}$. Even the $Q^{2}$-dependence is not that surprising; after all it is in general expected for any quantum field theory (e.g., QED) where virtual quanta renormalize the strength of the probe. What is interesting about QCD is the role that gluon self-interactions play in the behavior of the running coupling. With the boundary conditions of bound states on one side (hadrons, $Q^{2} \approx \Lambda_{Q C D}^{2}$ ) and of observable constituents on the other side (quarks, $Q^{2} \rightarrow \infty$ ), the stage is set for an asymptotically free non-Abelian gauge 
field theory.

The fact that the value of the strong coupling constant depends on the size of the probe used to measure it has many interesting implications and complications. Among these is the applicability of the perturbative calculations used to measure $\alpha_{s}$. Care must be exercised in insuring that the calculations used are valid in the regime of interest. Another complication is the fact that, due to the QCD ansatz of color-singlet states, the inherently strong hadronization process takes place. This process is a long-distance process, and thus, non-calculable in perturbative QCD. A qualitative understanding exists nevertheless, and allows us to model these low- $Q^{2}$ regimes. An optimist would say that this 'fuzzy' picture that QCD presents to the experimenter is just a symptom of the richness of the theory. This is to be contrasted with measurements in the Electroweak theory, where the precise determination of the coupling constants yields experimentally achievable sensitivities to new physics ${ }^{1}$.

In this investigation, we measured the value of $\alpha_{s}$ by determining the amount of high energy gluon radiation emitted by the primary quarks (anti-quarks) created in the decay of the $Z^{0}$. These primary quarks and gluons, in their race to 'dress-up' their color degrees of freedom, form cones or 'jets' of particles which eventually decay and interact with a detector. We studied these interactions to reconstruct the initial parton configurations, and together with perturbative calculations of jet-fractions, measured the value of $\alpha_{s}$. In this context, then, the study of jets provided an ideal place to investigate the quasi-free regime of parton de-confinement (high- $Q^{2}$ limit).

\section{Experimental Motivation}

Although the strong coupling constant has been measured extensively in $e^{+} e^{-}$annihilation in the energy range $\sqrt{s}=14-92 \mathrm{GeV}$, most of these measurements have been performed by using charged particles only. We performed a complementary measurement by using a calorimeter only; thus, both charged and neutral particles were used.

Why, then, another measurement of $\alpha_{s}$ ?

We will see that, even though the SLD calorimeter was not fully understood during the 1992 run, calorimeters provide very robust measurements of global event observables. In our case we used jet-fractions. In addition, the way in which calorimeters work mimic the requirements for an observable to be perturbatively calculable. One can think of calorimeters as being both infrared and collinear safe ${ }^{2}$.

\footnotetext{
${ }^{1}$ This is more a comment on the calculational techniques than on the theory itself.

${ }^{2}$ In the next chapter we will discuss these issues for QCD. In a calorimeter, the infrared cutoff is naturally provided by the finite number of cells while the collinear 'safety' is a direct result of linearity.
} 


\subsection{Thesis Outline}

This thesis presents a measurement of the strong coupling constant $\alpha_{s}$ in $e^{+} e^{-}$annihilation at $E_{c m}=91.55 \mathrm{GeV}$. The thesis is divided into the following sections:

- Chapter 1 In the rest of this chapter, we will summarize the Standard Model.

- Chapter 2 We will briefly review QCD, especially in $e^{+} e^{-}$annihilation. We will also discuss the theory and the phenomenology behind the measurement.

- Chapter 3 The experimental apparatus used in this measurement will be described with special emphasis on the calorimeter system.

- Chapter $4 \mathrm{~A}$ review of the performance and the calibration of the calorimeter system will be presented.

- Chapter 5 The triggering and the selection of hadronic events will be discussed. A discussion of backgrounds and efficiencies will also be presented.

- Chapter 6 Having gathered the necessary tools from chapters 2 to 6 , we present the actual measurement in this chapter. A discussion of the results and the systematic errors is also included.

\subsection{Electroweak Interactions}

In $e^{+} e^{-}$colliders with energies $\lesssim 100 \mathrm{GeV}$, the main $q \bar{q}$ production mechanism is through electroweak interactions ${ }^{3}$. We therefore present a brief summary of the features of the Electroweak theory which are relevant to our measurement.

The theory of Electroweak Interactions $[1,2,3]$ is a field theoretic description of the unification of Quantum Electrodynamics (QED) and an extension of Fermi's theory of weak decays. The prediction and discovery by direct production [4] of the charged and neutral carriers of the weak force in 1983 has so far been one of its greatest achievements.

In the Electroweak theory the charged carriers, the $W^{ \pm}$bosons, are maximally parity violating with a vertex factor proportional to $\gamma_{\mu}\left(1-\gamma_{5}\right)$. The $Z^{0}$ boson is the neutral carrier of the weak force. It has the same quantum numbers of the photon (and hence interferes with it) but has a hybrid chirality and a vertex term proportional to $\gamma_{\mu}\left(C_{V}^{f}-\gamma_{5} C_{A}^{f}\right)$, where the $C_{V}^{f}$ and $C_{A}^{f}$ are predicted by the Standard Model for each fermion type $f$. The theory is based on the $\mathrm{SU}(2)_{L} \times \mathrm{U}(1)_{Y}$ symmetry of an isotriplet of vector fields that couple to a weak isospin current (left-handed) and an additional vector field that couples to a weak hypercharge current. In the symmetry

\footnotetext{
${ }^{3}$ In hadron colliders, for example, the dominant production mechanism is QCD itself.
} 


\begin{tabular}{||l|r|r|r|r|r|r||}
\hline fermion & $Q_{f}$ & $T$ & $T_{3}^{f}$ & $Y$ & $C_{V}$ & $C_{A}$ \\
\hline$\nu_{l}$ & 0 & $\frac{1}{2}$ & $\frac{1}{2}$ & -1 & 0 & $\frac{1}{2}$ \\
$l^{-}$ & -1 & $\frac{1}{2}$ & $-\frac{1}{2}$ & -1 & $-\frac{1}{2}$ & $-\frac{1}{2}+2 \sin ^{2} \theta_{W}$ \\
$u$ & $\frac{2}{3}$ & $\frac{1}{2}$ & $\frac{1}{2}$ & $\frac{1}{3}$ & $\frac{1}{2}$ & $\frac{1}{2}-\frac{4}{3} \sin ^{2} \theta_{W}$ \\
$d$ & $-\frac{1}{3}$ & $\frac{1}{2}$ & $-\frac{1}{2}$ & $\frac{1}{3}$ & $-\frac{1}{2}$ & $-\frac{1}{2}+\frac{2}{3} \sin ^{2} \theta_{W}$ \\
\hline
\end{tabular}

Table 1.1: Electroweak quantum numbers and couplings to the $Z^{0}$.

breaking mechanism for mass generation these bosons mix into physical states with the parameter $\sin \theta_{w}$. The couplings to the $Z^{0}$ are given by:

$$
\begin{aligned}
& C_{V}^{f}=T_{f}^{3}-2 \sin ^{2} \theta_{W} Q_{f} \\
& C_{A}^{f}=T_{f}^{3}
\end{aligned}
$$

where the $T_{f}^{3}$ is the third component of weak isospin and $Q_{f}$ is the fermion charge in units of $e$. Table 1.1 summarizes these quantities.

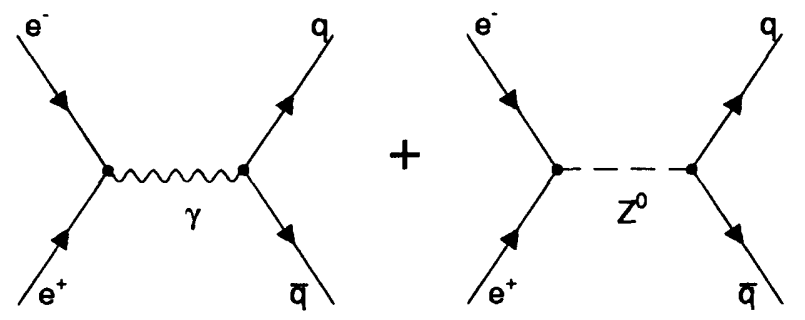

Figure 1-1: Tree level Feynman diagrams for quark $(q)$ and antiquark $(\bar{q})$ production.

In $e^{+} e^{-}$annihilation, and in the vicinity of $\sqrt{s} \approx M_{Z^{0}}$, the production cross section for fermion pairs $(f \bar{f})$ is enhanced due to $Z^{0}$ resonance production. As Figure 1-2 shows, the total cross section is just the overlap of a continuum term (QED) and a Breit-Wigner shaped resonance ( $Z^{0}$ production). The Feynman diagrams for this process are shown in Figure 1-1. Interference effects are also included. The production mechanism for $q \bar{q}$ in $e^{+} e^{-}$annihilation is then the same mechanism as that for leptons but with a modified couplings to the $Z^{0}$. 
The cross section for hadronic production at the $Z^{0}$ is given (to $\left.\mathcal{O}\left(\alpha_{s}^{3}\right)\right)$ by $[5,6]$ :

$$
\sigma_{\text {tot }}^{h}=\sigma_{q}\left[1+1.05 \frac{\alpha_{s}\left(M_{Z}\right)}{\pi}+(0.9 \pm 0.1)\left(\frac{\alpha_{s}\left(M_{Z}\right)}{\pi}\right)^{2}-13\left(\frac{\alpha_{s}\left(M_{Z}\right)}{\pi}\right)^{3}+\cdots\right] \text {, }
$$

where $\sigma_{q}$ is the tree level hadronic cross section,

$$
\sigma_{q}=\frac{4 \pi \alpha_{e m}^{2}}{3 s}\left(\frac{11}{3}\right) R_{E W}
$$

and where $R_{E W}$ is the ratio of hadronic to leptonic widths calculated with $\alpha_{s}=0$ at a center-of-mass energy of $\sqrt{s}=M_{Z}$.

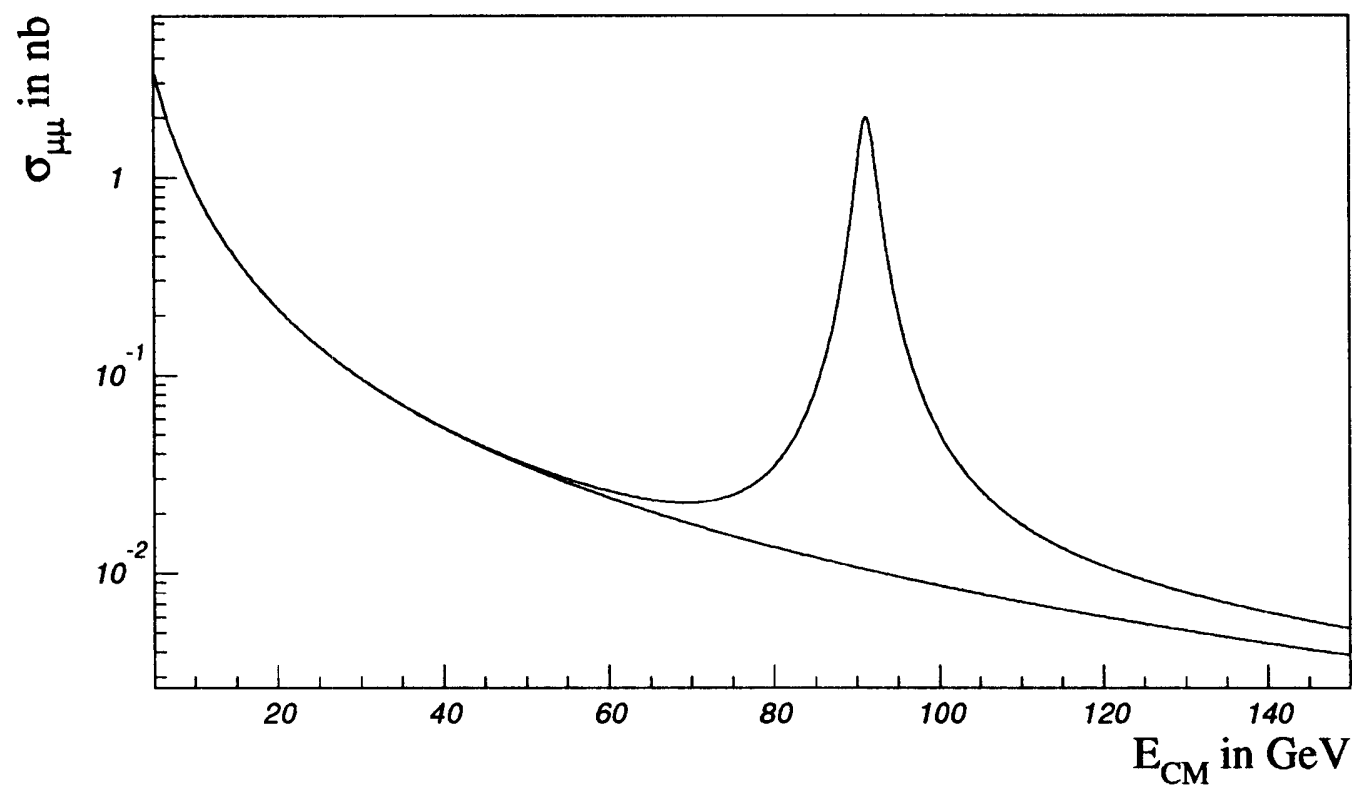

Figure 1-2: Cross section for $e^{+} e^{-} \rightarrow \mu^{+} \mu^{-}$, showing both the continuum contribution (bottom line) and the resonance contribution. 


\section{Chapter 2}

\section{Quantum Chromodynamics}

\subsection{Development of QCD}

The basic building blocks of the modern interpretation of strong interaction phenomena were established in 1964 when Gell-Mann and Zweig $[7,8]$ independently developed the quark model of hadrons. This model, based on a $S U(3)$ flavor symmetry of spin- $\frac{1}{2}$ and fractionally charged particles, postulated that hadrons had a sub-structure. The quark model was an attempt to order the "table of hadrons" of the time. At their inception, the quarks were just a mathematical tool to keep track of the various hadron species - a sort of "bookkeeper of symmetries". This model, however, was not without problems.

The totally symmetric same-quark states $\left(\Delta^{++}, \Delta^{-}, \Omega^{-}\right.$baryons) were forbidden by the Pauli exclusion principle in the context of the quark model alone. This problem was solved [9] by postulating yet another degree of freedom for the quarks (in addition to spin and flavor) which was called color. As it turned out, the idea of color was later to become, from a field theoretic point of view, the central idea of "full-blown" QCD.

The discovery of the scaling of structure functions in 1968 in the SLAC-MIT experiments [10] of deep inelastic electron-nucleon scattering provided the first indication of point-like structure inside the proton. In these experiments, a high- $Q^{2}$ (i.e., high spatial resolution) electron probe was used to examine the proton. It was found that as the resolution of the probe increased, the structure functions associated with the proton changed from being those of a disc of finite extent to those of a composite structure of point-particles. This property of Bjorken scaling immediately led to the development of Feynman's parton model of hadrons. This model was what today we would consider as zeroth-order QCD even though, initially, the connection to Gell-Mann's quarks had not been made.

Later studies of the spin and charge content of the proton led to identifying these partons as the quarks. The observation that quarks behaved more and more like point-particles as the $Q^{2}$ of the probe increased was crucial to the later development 
of asymptotic freedom in QCD. The quark-parton model was then extended to consist of 3 quark constituents and a "sea" of quark-antiquark pairs bound together by neutral "gluons". The observation that one-half of the nucleon momentum was carried by the quarks and the (later) observation of scaling violations (higher-order deviations of the parton model) confirmed this view.

During the 60's and early 70's many models $[11,12]$ of the nucleon were put forth to explain Bjorken scaling. One by one, with exception of the modified version of Feynman's quark-parton model, they were all discarded by experiment. The one last hurdle to the parton picture was then the fact that quarks had never been observed in a free state. In addition, Feynman's model provided no explanation for the strong interaction phenomena which led to the production of hadrons.

The breakthrough came when Gross, Wilczek, and Politzer [13, 14] re-examined the non-Abelian gauge field theories originally proposed in the 50's by Yang and Mills [15]. Using renormalization group methods to calculate charge renormalization to one loop, they showed that the Yang-Mills formulation had the desired property of asymptotic freedom. In one stroke they solved the puzzle by explaining a strong interaction at large distance with quasi-free behavior at small distances. At that time the renormalizability of non-Abelian gauge field theories had already been proven and their quantization achieved [16]. A coherent picture for the dynamics of the theory was finally achieved when the symmetries of the noncommutative gauge groups was identified with an exact color symmetry.

Quantum Chromodynamics was thus born. It was a great achievement that the constituent and dynamical aspects of the quark picture had been reconciled. The color degrees of freedom had a dual role: it solved the counting problem in the constituent picture of quarks and, in the context of a gauge theory, its invariance provided the dynamical mechanism for the strong force. In the 60's, many people argued that the strong interaction would never be described by the methods of perturbative field theory. For the most part, that argument still holds. Perturbative QCD (PQCD) is inapplicable in the $Q^{2}$ regimes of large coupling strengths. However, as new, higher energy accelerators were built to probe deeper into the nucleon, the asymptotically free regime of QCD was unleashed. Once the quasi-free approximation for quarks could be reached, then PQCD became applicable and physical observables calculable. This limit is essential for our measurement.

\subsection{The Theory}

QCD is rich, both theoretically and experimentally. The complicated structure of its vacuum, the nature and scale of the strong coupling $g_{s}$, and the fact that it is a young theory only underscore the importance of testing it experimentally. The program of calculations and experiments to test QCD is vast and vigorous; they include tests of collective phenomena and the vacuum at heavy ion colliders, axion searches (strongCP problem), lattice-gauge calculations, studies of hadron production, and studies of 
the asymptotic limit (measurement of perturbatively calculable observables).

In the following we will concentrate on the aspects of QCD applicable to our measurement. These include perturbative QCD in $e^{+} e^{-}$annihilation and the related low- $Q^{2}$ phenomenology.

\section{The Lagrangian}

Quantum Chromodynamics [17] is the local non-Abelian gauge field theory of colored quarks and gluons. The quarks are point-like spin- $\frac{1}{2}$ particles, which in addition to the Electroweak quantum numbers of Table 1.1, carry the color charge. The strong color charge arises from the exact and local $S U(3)$ gauge symmetry and is thus conserved. Just like in QED, where the requirement of local gauge invariance gives rise to the photon field, in QCD the gluon field arises of such invariance to mediate the strong force. Unlike QED however, where the symmetry group is the Abelian group $U(1)$, the non-commutative properties of $S U(3)_{c}$ imply that the gluon force mediators are themselves carriers of the color charge. Gluon-gluon couplings are thus allowed.

Following the notation of [16], the classical $^{1}$ QCD Lagrangian is given by:

$$
\mathcal{L}_{Q C D}=-\frac{1}{4} F_{\alpha \beta}^{a} F_{a}^{\alpha \beta}+\sum_{\text {flavors }} \bar{q}_{k}\left(i \gamma^{\mu} D_{\mu}-m_{k}\right) q_{k}
$$

where,

$$
F_{\mu \nu}^{a}=\partial_{\mu} A_{\nu}^{a}-\partial_{\nu} A_{\mu}^{a}+g_{s} f^{a b c} A_{\mu}^{b} A_{\nu}^{c}
$$

is the field strength tensor from the gluon field $A_{\mu}^{a}$ and where,

$$
D_{\mu}=\partial_{\mu}-i g_{s} T^{a} A_{\mu}^{a}
$$

is the generalized covariant derivative. In equations $2.1,2.2$, and 2.3 , ' $a$ ' is the color index $a=1,2, \ldots, 8, f^{a b c}$ are the $S U(3)_{c}$ group structure constants, $T^{a}$ are the $S U(3)_{c}$ generators, and the $q_{k}\left(\bar{q}_{k}\right)$ are the quark (anti-quark) spinor fields of flavor $k$.

We can see from Equations 2.1-2.3 that the only free parameters in QCD are the dimensionless coupling $g_{s}$ and the quark masses $m_{k}$. However, to a good approximation [18], QCD displays a chiral symmetry $S U(3) \times S U(3)$ in the limit that the quark masses $m_{u}=m_{d}=m_{s}=0$. So, at sufficiently high energies, we see that there is no explicit parameter setting a mass scale ( $g_{s}$ is dimensionless). It is the renormalization scale parameter $\mu$ that sets a mass scale for the theory by specifying at which point $g_{s}$ is renormalized.

\footnotetext{
${ }^{1}$ In order to quantize QCD (or any gauge theory) the gauge freedom must be fixed. This can be done by explicitly including gauge-fixing terms in Equation 2.1. In addition, unphysical degrees of freedom are removed by including a Faddeev-Popov "ghost" term.
} 


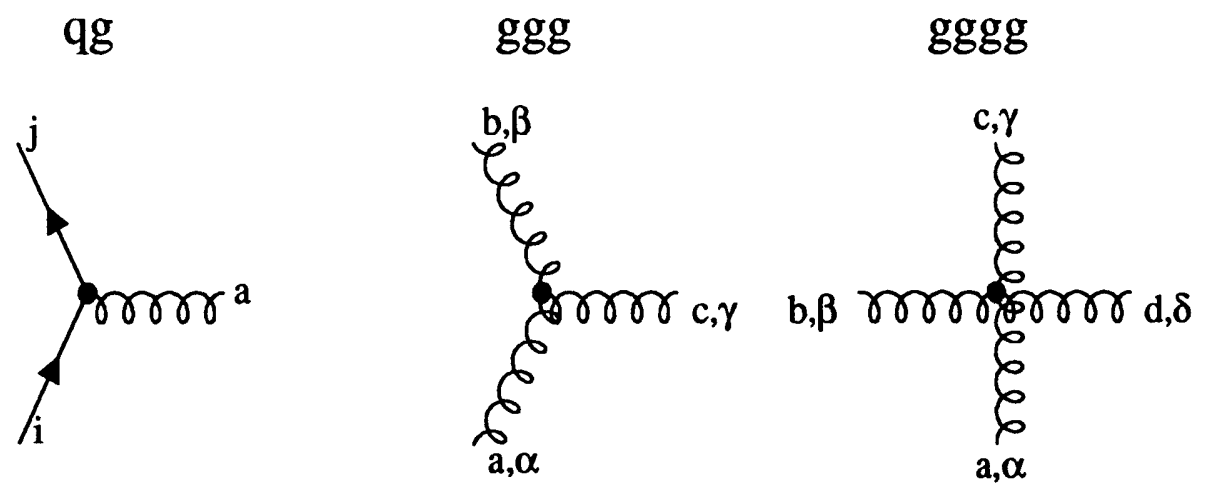

Figure 2-1: $\quad$ Some QCD interactions. The labels ' $i$ ' and ' $j$ ' are the quark color indices $(i, j=1,2,3)$, while $\mathrm{a}, \mathrm{b}, \mathrm{c}$, and $\mathrm{d}$ are the gluon color labels $(=1,2, \ldots, 8)$.

\section{Interactions in QCD}

We can almost read-off the vertex factors from Equations 2.1-2.3. One thing to note, when comparing QCD with QED, is that the non-trivial Lie algebra of $S U(3)_{c}$ introduces an extra term proportional to $f^{a b c}$ in Equation 2.2. This term is the one that gives rise to the gluon-gluon interactions and is a direct result of the non-Abelian nature of QCD. In terms of the fundamental constant $g_{s}$, we have for the physical (excluding the ghost terms) vertex factors:

$$
\begin{aligned}
q g & -i g_{s} \gamma^{\mu} T_{i j}^{a} \\
g g g & -g_{s} f^{a b c} V_{\alpha \beta \gamma} \\
g g g g & -i g_{s}^{2} W_{\alpha \beta \gamma \delta}^{a b c d}
\end{aligned}
$$

where the $V$ and $W$ are functions of the leg momenta and can be found in reference [19]. These interactions are diagramed in Figure 2-1. In our study we will encounter the first two of these interactions; the gggg coupling is out of our reach since it is of higher order than the present $\mathcal{O}\left(\alpha^{2}\right)$ calculations because at tree level,

$$
\mathcal{O}\left(e^{+} e^{-} \rightarrow 5 \text {-jets }\right)=\left(g_{s} g_{s}^{2}\right)^{2}=\mathcal{O}\left(\alpha_{s}^{3}\right) .
$$

In the above the 5 jets originate from the $g g g g$ coupling with one of the legs attached to the $q \bar{q}$ pair. More on that later.

\subsubsection{Renormalization}

In a Quantum Field Theory (QFT) like QCD, loop integrals are in general divergent. These divergencies are rooted in the 'locality' assumption of QFT: that interactions between two objects occur at the same space-time point (point-like interactions). However, a renormalizable theory is one that allows the divergencies to be absorbed 
into the physical parameters of the Lagrangian via a finite renormalization program. As mentioned previously, QCD has been shown to be renormalizable.

In order to visualize the above, it is useful to consider the case of mass renormalization in QED [20]. If we divide the QED equivalent of Equation 2.1 into a free and interacting part,

$$
\mathcal{L}=\mathcal{L}_{0}+\mathcal{L}_{\text {int }},
$$

and consider that electrons are stable and observable, then we would expect that $S_{e \rightarrow e}$ (the matrix element for electron $\rightarrow$ electron transition) should be 1. In fact, since electrons undergo self-interactions (emission and absorption of virtual photons), $S_{e \rightarrow e} \neq 1$. But we know from experience that if we "watch" an electron at the characteristic distance $d \approx \sqrt{1 / \mu^{2}}$ (e.g, Thompson scattering with $\mu^{2} \rightarrow 0$ ) we can measure a physical mass $m_{e}$. So in order to recover $S_{e \rightarrow e}=1$ for the free Lagrangian, we have to modify the decomposition in Equation 2.4 by adding a $\delta m$ term to the bare electron mass and subtract it from $\mathcal{L}_{\text {int }}$. Since we have "observed" the electron at a particular distance, this counter-term subtraction has been explicitly performed ${ }^{2}$ at the scale $Q^{2}=\mu^{2}$.

This re-shuffling of terms, the renormalization procedure, is just a response to the fact that the quantities postulated in the "bare" (unrenormalized) Lagrangian do not correspond to physical observables when interactions are present. This procedure is not unique.

\section{Dimensional Regularization}

Before carrying out the renormalization procedure, the infinities of the theory must be identified and regularized. This is usually accomplished by re-writing the Lagrangian (or any quantity being calculated) with an explicit cut-off; in the limit that this cutoff vanishes the original expression is then recovered. Of the various regularization schemes, the most convenient one for QCD is dimensional regularization [21]. This scheme is especially suited for QCD as it respects gauge invariance and makes it unnecessary to introduce additional invariance-restoring counter terms. In this procedure, the infinities are regulated by continuing the dimensionful expressions in the Lagrangian (and subsequent integrals) to $n=4-2 \epsilon$. In order to keep the coupling $g_{s}$ dimensionless, the replacement $g_{s} \rightarrow \mu^{\epsilon} g_{s}$ is made throughout. The arbitrary parameter $\mu$ is not specified with the exception that it has the units of mass; thus an explicit dependence on $\mu$ is introduced into the rescaled $g_{s}: g_{s}=g_{s}(\mu)$. The infinities are then explicitly re-expressed as poles in $(1 / \epsilon)^{n}$. Of course, at this stage nothing has changed: the original divergent expression is still obtained in the limit $\epsilon \rightarrow 0$.

\footnotetext{
${ }^{2}$ This is one difference between QED and QCD: since electrons are observable, an unambiguous renormalization scale can be chosen whereas QCD offers no such free states.
} 


\section{Renormalization Schemes and Conventions}

In this section, we follow the treatment and conventions of Duke and Roberts [22] and Muta [16].

One can always write any QCD perturbatively calculated observable as,

$$
R(g)=r_{0}+r_{1}\left(\frac{g^{2}}{4 \pi}\right)+r_{2}\left(\frac{g^{2}}{4 \pi}\right)^{2}+\cdots
$$

where $g$ is the strong coupling $\alpha_{s}=g^{2} / 4 \pi$, and where the $r_{i}$ are calculable $i$-th order coefficients. These coefficients (as we will see in the specific example of jet rates later) are in general ultraviolet divergent and are controlled by a specific regularization and renormalization program. We have said that renormalization amounts to a reshuffling of terms in the Lagrangian; this reshuffling can be done in a infinite number of ways. If we attach the label ' $a$ ' to both the $r_{i}$ and $g$ in Equation 2.5 denoting one of the particular renormalization conventions, we write,

$$
R^{a}\left(g_{a}\right)=r_{0}^{a}+r_{1}^{a}\left(\frac{g_{a}^{2}}{4 \pi}\right)+r_{2}^{a}\left(\frac{g_{a}^{2}}{4 \pi}\right)^{2}+\cdots+r_{n}^{a}\left(\frac{g_{a}^{2}}{4 \pi}\right)^{n}
$$

where we have explicitly truncated the series expansion at the $n$-th order. But we know that in real life observables yield definite results that do not depend on any renormalization convention. We would then expect that for a different renormalization convention $R^{a}\left(g_{a}\right) \approx R^{b}\left(g_{b}\right)$-at least to the maximum order of the calculation. We can rephrase this by saying that assuming we have an $n$-th order calculation of Equation 2.5 available, this last requirement can be written ${ }^{3}$ as [22],

$$
\left[R\left(g^{2} / 4 \pi\right)\right]_{n}^{a}-\left[R\left(g^{2} / 4 \pi\right)\right]_{n}^{b}=\mathcal{O}\left(\left[g^{2} / 4 \pi\right]^{n}\right)
$$

where $n$ denotes the order at which the series 2.5 is truncated and $a$ and $b$ denote different renormalization schemes. We see then that as $n \rightarrow \infty$, the results of the perturbative expansions in two different renormalization conventions given by Equations 2.5 and 2.6 agree. We will encounter the effects of the truncation in Equation 2.6 in our measurement in the form of renormalization scale uncertainties.

Examples of various renormalization conventions are given in reference [22]. In our work we choose to use the modified minimal subtraction scheme, which together with the dimensional regularization procedure, completes the renormalization program. In this scheme the $1 / \epsilon$ poles in the perturbative expansion are subtracted via Lagrangian counter terms. The additional terms ' $\ln 4 \pi-\gamma_{E}$ ' ( $\gamma_{E}$ is Euler's constant), relics of the dimensional regularization, are also subtracted.

We should mention that some authors (e.g., Stevenson [23], Brodsky et al. [24],

\footnotetext{
${ }^{3}$ We also use the result [16] that the two couplings can be related through a finite renormalization transformation. This in turn implies that $r_{0}$ and $r_{1}$ are scheme independent and that the higher order $r_{i}$ can be converted from one scheme to another.
} 
among others) advocate specific schemes to reduce the renormalization scheme ambiguity that arises due to the uncalculated terms in Equation 2.7. The use of these schemes is generally called 'optimized perturbation theory'. The PMS scheme, for example, advocates evaluating an observable at the scale $Q^{\star}$ such that $\partial R\left(g, Q^{\star}\right) / \partial Q=$ 0 . Such a scale would presumably minimize the effects of missing higher orders by artificially reducing the sensitivity to them. Other people [14], however, have argued strongly against some of these schemes.

\section{The $\beta$-function and the Running of $\alpha_{s}$}

In this section we will investigate the consequences of renormalizing the strong coupling $g_{s}$. We will denote the bare (unrenormalized) coupling by $g_{b}$ and the renormalized coupling by $g_{s}$. Good references for this section are Gross and Wilczeck [25] and Field [19].

The leading order corrections to the $q \bar{q} g$ vertex are shown in Figure 2-2. The rightmost column in the figure shows the amplitude for the sum of amplitudes in each row. The ' $Z_{i}$ ' factors are the renormalization factors [19] absorbing the ultraviolet divergencies of the corresponding amplitudes ${ }^{4}$. In order to extract the renormalized strong coupling, the corrections in Figure 2-2 are added to yield [19],

$$
\begin{aligned}
-i g_{s} \gamma_{\mu} T_{a} & =\frac{1}{Z_{2} \sqrt{Z_{3}}}\left[1+\left(Z_{1}^{-1}-1\right)-2\left(Z_{2}^{-1}-1\right)+\left(Z_{3}-1\right)\right]\left(-i g_{b} \gamma_{\mu} T_{a}\right) \\
& =\frac{Z_{2} \sqrt{Z_{3}}}{Z_{1}}\left(-i g_{b} \gamma_{\mu} T_{a}\right),
\end{aligned}
$$

where we can now read off the the renormalized coupling in terms of the bare coupling,

$$
g_{s}=\frac{Z_{2} \sqrt{Z_{3}}}{Z_{1}} g_{b}
$$

The $Z_{i}$ are written in terms of the coupling $g_{s}$, the dimensional regularization mass $\mu$, the explicit divergent parts $1 / \epsilon$, and some QCD factors. Combining these into Equation 2.9 to order $g_{s}^{2}$ gives [26],

$$
g_{s}=\left[1+\frac{g_{s}^{2}}{16 \pi^{2}} \beta_{0} \frac{1}{2}\left(\frac{1}{\epsilon}+\ln (4 \pi)-\gamma\right)\right] \mu^{-\epsilon} g_{b}
$$

where the above is given in the $\overline{M S}$ scheme and where,

$$
\beta_{0} \equiv \frac{11}{3} N_{c}-\frac{2}{3} n_{f}
$$

In the above, $N_{\mathrm{c}}=3$ is the number of colors and $n_{f}$ is the number of quark flavors.

\footnotetext{
${ }^{4}$ By convention, $i=1$ corresponds to the vertex correction, $i=2$ corresponds to fermion selfenergy corrections, and $i=3$ corresponds to the gluon propagator corrections.
} 
We now impose the requirement that, since $\mu$ is an arbitrary parameter, $g_{b}$ must be independent of $\mu$, or,

$$
\mu \frac{d g_{b}}{d \mu}=\mu\left[\frac{\partial}{\partial \mu}+\frac{\partial g_{s}}{\partial \mu} \frac{\partial}{\partial g_{s}}\right] g_{b}=0
$$

Applying this last result to Equation 2.10 and performing the renormalization subtraction gives,

$$
\mu \frac{\partial g_{s}}{\partial \mu}=-\frac{\beta_{0}}{16 \pi^{2}} g_{s}^{3}
$$

and the renormalization group (RGE) result [27] is obtained. In this last equation, the renormalization procedure has been carried out using the $\overline{M S}$ prescription. A very important result is already evident from Equation 2.11. Notice that for $\beta_{0}>0$ (equivalently, $n_{f} \leq 16$ ), Equation 2.11 implies that the coupling $g_{s}$ decreases with an increase in the energy scale. This very important property is called asymptotic freedom and allows us to use perturbation theory at high energies in QCD. It is important to note that this behavior ('negative $\beta$-function') is solely due to the non-Abelian nature of QCD (cf., QED has a 'positive $\beta$-function').

Writing Equation 2.11 to higher orders [28] and using $\alpha_{s}=g_{s}^{2} / 4 \pi$, we quote,

$$
\mu \frac{\partial \alpha_{s}}{\partial \mu}=-\frac{\beta_{0}}{2 \pi} \alpha_{s}^{2}-\frac{\beta_{1}}{8 \pi^{2}} \alpha_{s}^{3}-\cdots,
$$

where $\beta_{1}=102-\frac{38}{3} n_{f}$ and where the right side of the above equation is generally called the $\beta$-function. The coefficients $\beta_{0}$ and $\beta_{1}$ are renormalization scheme independent; in general, however, higher order coefficients do depend on the renormalization scheme used.

Perturbative QCD does not tell us the 'absolute value' of $\alpha_{s}$, it just tells us how it behaves through Equation 2.12. In fact, what is missing from the differential equation, and is not given by the theory, is a boundary condition to completely specify the behavior of $\alpha_{s}$. In QED the Thompson limit $\left(Q^{2} \rightarrow 0\right)$ provides a natural boundary condition defined in terms of an observable object-an electron. In QCD the convention [6] is to introduce a renormalization scheme dependent mass parameter $\Lambda$,

$$
\ln \frac{Q^{2}}{\Lambda^{2}}=\int_{\infty}^{\alpha_{s}(Q)} \frac{d x}{\beta(x)},
$$

where $\beta$ is the function defined by Equation 2.12. In the following, and for simplicity, the one-loop approximation (Equation 2.11) will be used. One can see from Equation 2.13 that the chosen boundary condition is $\alpha_{s}(\Lambda)=\infty$.

We can proceed with Equation 2.13 to obtain a closed form for $\alpha_{s}$,

$$
\alpha_{s}(Q)=\frac{4 \pi}{\beta_{0} \ln \left(Q^{2} / \Lambda^{2}\right)} .
$$

Now asymptotic freedom is fully evident in Equation 2.14: as $Q^{2} \rightarrow \infty, \alpha_{s}(Q) \rightarrow 0$. 


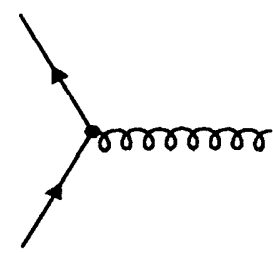

$$
-\lg _{b} \gamma_{\mu} T_{a}
$$
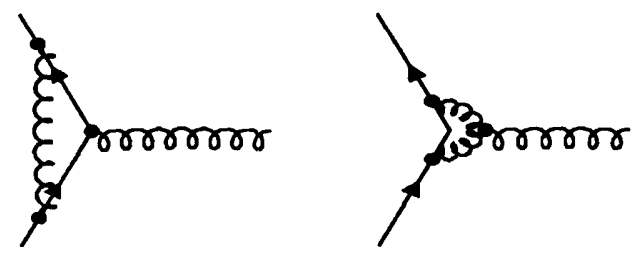

$$
-\mathrm{ig}_{b} \gamma_{\mu} \top_{a}\left(Z_{1}^{-1}-1\right)
$$
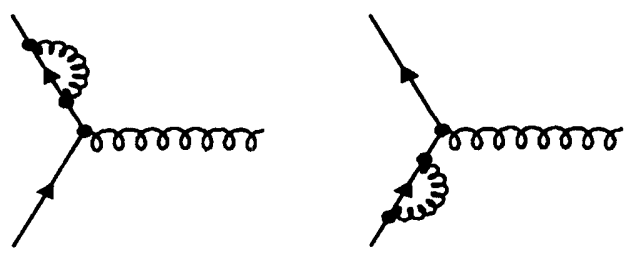

$$
2 \mathrm{ig}_{b} \gamma_{\mu} T_{a}\left(Z_{2}^{-1}-1\right)
$$
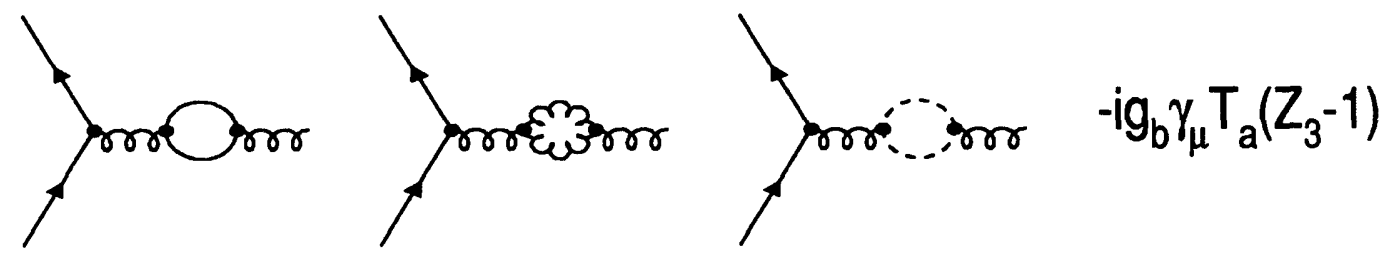

Figure 2-2: Leading order corrections. Top to bottom: the bare vertex, vertex correction, quark self-energy correction, and gluon propagator corrections. Lines are quarks, wavy lines are gluons, and dashed lines are ghosts. $g_{b}$ stands for the bare coupling and $T_{a}$ is the $S U(3)$ generator.

We now see the role of the QCD parameter $\Lambda$ : it acts as a vague limit at which $\alpha_{s}$ becomes strong, and it sets the scale at which $\alpha_{s}$ 'runs' (i.e., 'how fast' it runs). It is this parameter which we will determine in this analysis.

It is instructive to paint a physical picture of asymptotic freedom. In QED, the vacuum polarization (virtual $e^{+} e^{-}$pairs) shields the bare charge $e_{b}$ into a renormalized charge $e\left(Q^{2}=0\right)$, defined at large distances. As one probes this polarization cloud $\left(Q^{2} \rightarrow \infty\right)$, this screening effect becomes less pronounced and we see a larger effective charge. In QCD the reverse is true. We still have color charge screening due to $q \bar{q}$ vacuum polarization, however, now the gluons also carry charge. This means that our test particle (the 'source' of color charge) can now radiate its charge away. As we get closer to it, this radiation becomes more prominent and we have less of a chance of localizing the original test charge. Thus, the effective color charge in QCD become weaker as $Q^{2} \rightarrow \infty$. We may be tempted to extend this picture to the confinement limit but in that limit our picture of single gluons and charges breaks down (non-perturbative regime). 


\section{Some Results We'll Need}

Before leaving this section, we will quote some results which will be used later in the analysis. The second order solution (next-to-leading order) to the $\beta$-function is [28],

$$
\alpha_{s}(Q)=\frac{12 \pi}{\left(33-2 n_{f}\right) \ln \left(Q^{2} / \Lambda^{2}\right)}\left[1-\frac{6\left(153-19 n_{f}\right)}{\left(33-2 n_{f}\right)^{2}} \frac{\ln \left[\ln \left(Q^{2} / \Lambda^{2}\right)\right]}{\ln \left(Q^{2} / \Lambda^{2}\right)}\right]
$$

where, again, we will use $\Lambda=\Lambda_{\overline{M S}}$. An alternative solution to Equation 2.12 with the $\beta$-function truncated at second order is [28],

$$
\frac{\beta_{0}}{2 \pi} \ln \frac{\mu}{\Lambda}=\frac{1}{\alpha_{s}(\mu)}+\frac{\beta_{1}}{4 \pi \beta_{0}} \ln \left(\frac{\alpha_{s}(\mu)}{\alpha_{s}(\mu)+\frac{4 \pi \beta_{0}}{\beta_{1}}}\right)
$$

which enables us to easily present the results in terms of $\Lambda_{\overline{M S}}$. Equations 2.15 and 2.16 are equivalent to $\mathcal{O}\left(\alpha_{s}^{2}\right)$ and may thus be used interchangeably; however, one must be careful in being consistent in their usage. The conversion between $\Lambda_{\overline{M S}}$ from Equations 2.15 (labelled ' $A$ ') and 2.16 (labelled ' $\mathrm{B}$ ') is,

$$
\Lambda_{A}=1.076 \Lambda_{B}
$$

where $\Lambda=\Lambda_{\overline{M S}}$ is for five active flavors.

One may freely convert the $\Lambda$ parameters between different renormalization schemes and different number of flavors. Two renormalization schemes are related by a 1-loop calculation [6]. By imposing continuity at the boundary conditions of the flavor thresholds in Equation 2.15, the $\Lambda_{\overline{M S}}$ for different $n_{f}$ can also be calculated [29].

It is important to note that in order to have a meaningful determination of $\Lambda$, at least a next-to-leading order calculation must be used [30]. The reason is that to leading order (Equation 2.14) a scale change in $\Lambda$ of $\mathcal{O}(1)$ implies a change in $\alpha_{s}$ of $\mathcal{O}\left(\alpha_{s}^{2}\right)$. Thus to leading order, a determination of $\Lambda$ yields an effective $\Lambda_{\text {eff }}$ not related to the parameter of the theory $\Lambda_{\overline{M S}}$.

\subsection{Perturbative QCD in $e^{+} e^{-}$Annihilation}

\subsubsection{Experimental Developments}

The annihilation of $e^{+} e^{-}$provides a very clean environment for QCD studies. A nice feature of such colliders is that the center of mass system, except for initial state radiation, coincides with the laboratory reference system, making the job of untangling final states much easier.

The most direct manifestation of quarks or gluons in a quasi-free state is jets. In 1970, while considering various models for hadron distributions in $e^{+} e^{-}$annihilation, 
Bjorken and Brodsky [31] suggested the idea of "jets" as a possible manifestation of the parton structure of a heavy virtual photon. It was five years later, at the Mark II detector at SPEAR [32], that the first evidence for jets was obtained by observing an excess of low sphericity events at $\sqrt{s} \approx 7 \mathrm{GeV} .{ }^{5}$ In addition, from the $1+\cos ^{2} \theta$ distribution of the sphericity axis of the hadronic events, it was inferred that the produced quarks were spin- $\frac{1}{2}$ objects. This was a great triumph for QCD; in a completely different environment from the DIS experiments, it had been shown that spin- $\frac{1}{2}$ quarks were observable in the asymptotic limit.

The observation of gluon emission came later in 1979 [33]. Three jet events were observed in the experiments at the PETRA ring in DESY at an energy $\sqrt{s} \approx 30$ $\mathrm{GeV}$. This time, the separation of three-jet events from phase-space distributed events proved more difficult than with two-jet events. The problem was that it was no longer sufficient to separate the events into hemispheres - there was an ambiguity in defining the third jet. The fragmentation process was smearing the initial parton direction and thus made it impossible, on an event by event basis, to differentiate a true gluon jet from a fluctuation in the hadronization. This could only be shown statistically.

We still suffer from these hadronization effects. The smearing introduced by such effects and our lack of knowledge of these low- $Q^{2}$ phenomena will introduce a systematic uncertainty in our measurement of jet-rates. These effects will be discussed in more detail later.

\subsubsection{QCD Perturbative Predictions}

In this section we will briefly motivate and review the $\mathcal{O}\left(\alpha_{s}^{2}\right)$ matrix element calculations with the purpose of setting the stage for the actual measurement. Very good references for perturbative QCD are Kramer [26] (PQCD in $e^{+} e^{-}$) and Muta [16] (PQCD in general). We will closely follow Kramer's treatment.

\section{Some $e^{+} e^{-}$QCD Quantities}

In $e^{+} e^{-}$annihilation one can vaguely classify, in an experimental sense, ' $\alpha_{s}$-dependent' quantities as either being inclusive or kinematically distributed quantities. Example of inclusive observables are [5]:

- $R_{e^{+} e^{-}}$: hadronic fraction of the total cross section

- $\Gamma_{h} / \Gamma_{l}$ : the hadronic/leptonic fraction

- $R_{\tau}$ : hadronic fraction for $\tau$ leptons

By being inclusive, the measurement of the above quantities is fairly insensitive to the final state. For example, hadronization effects are especially suppressed. In addition,

\footnotetext{
${ }^{5}$ Sphericity, $S$, is defined in Appendix A.
} 
the available calculational techniques allow calculations of up to $\mathcal{O}\left(\alpha_{s}^{3}\right)$, making the above attractive candidates for the determination ${ }^{6}$ of $\alpha_{s}$. However, the dependence on $\alpha_{s}$ (in the above variables) enters as a QCD correction, and although known to third order, tends to be dominated by statistical errors.

Some examples of kinematically distributed quantities [34] are:

- Event shapes: thrust, oblateness, heavy jet mass

- Particle-inclusive quantities: energy-energy correlations (EEC), asymmetry of the EEC (AEEC), single particle spectra

- Jet quantities: jet rates, differential jet rates

The above observables are defined either in terms of single particles or in terms of clusters of particles. This implies, of course, that fragmentation uncertainties dilute the measurement. In addition, none of these quantities have been fully calculated to higher than $\mathcal{O}\left(\alpha_{s}^{2}\right)$ so far and thus the maximum achievable accuracy is less than for the inclusive quantities. Therefore, in general, inclusive quantities have smaller theoretical uncertainties than kinematically distributed quantities. There is one important advantage over the inclusive variables, though; the above quantities can in general be written as direct proportionalities with $\alpha_{s}$. Thus the experimental sensitivity is much higher.

From now on we will concentrate on the jet related quantities. We will briefly review the calculation of gluon radiation in $e^{+} e^{-}$annihilation and use it to predict jet rates. The fact that this prediction is a function of $\alpha_{s}$ will enable us to use it later on to extract a value of $\Lambda_{\overline{M S}}$ from the data.

\section{The Parton Final States to $\mathcal{O}\left(\alpha_{s}\right)$}

It is instructive to outline the issues involved in the calculation of $q \bar{q} g$ final states in $e^{+} e^{-}$annihilation to $\mathcal{O}\left(\alpha_{s}\right)$. These issues are representative of the ones encountered in the higher order calculations but are less encumbered by the algebra.

Up to $\mathcal{O}\left(\alpha_{s}\right)$, we can have at most three partons in the final state. The complete set of Feynman diagrams that contribute to this order are shown in Figures 1-1 and 23a ( $q \bar{q}$ tree level and 1-loop) and in Figure 2-3b ( $q \bar{q} g$ tree level). Each diagram in 2-3a carries an ultraviolet $(k \rightarrow \infty)$ divergence that cancels when the three diagrams are added. An additional divergence, this time infrared $(k \rightarrow 0)$, appears due to the masslessness of the gluons. However, the diagrams of Figure 2-3b display the same divergence with the opposite sign and thus cancel it in their sum. There is still one more related divergence to discuss but we first turn our attention to the nature of the cross section.

\footnotetext{
${ }^{6} \mathrm{~A}$ summary of $\alpha_{s}$ determinations is presented in Chapter 6; see Table 6.9
} 

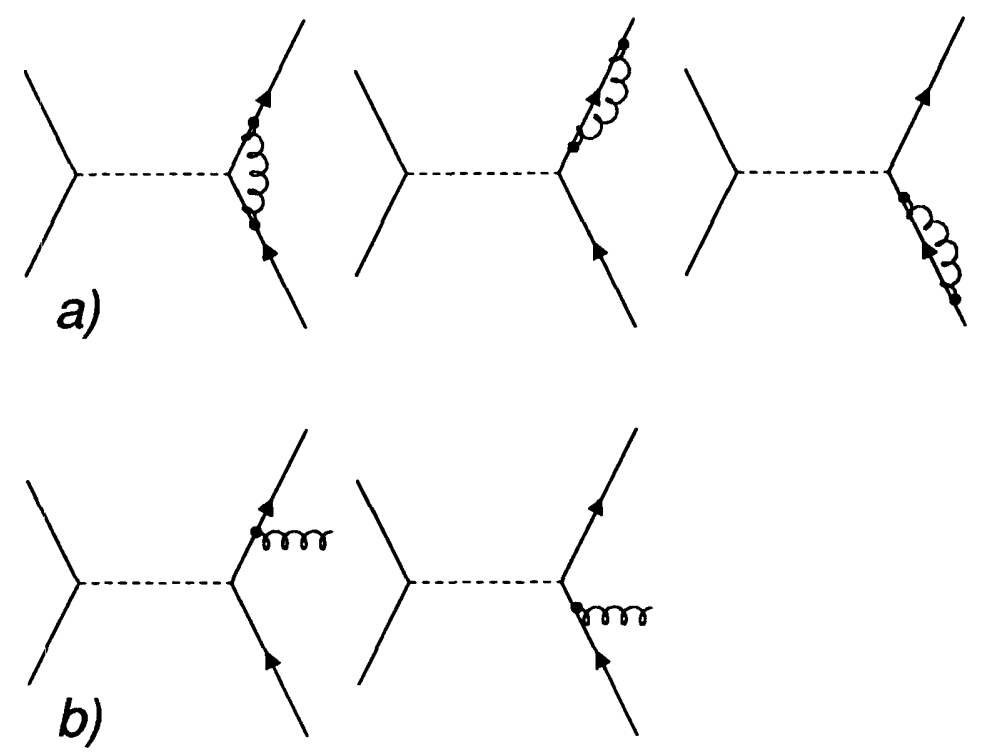

Figure 2-3: Diagrams contributing to $\mathcal{O}\left(\alpha_{s}\right)$ parton production. a) shows the 2-parton final state virtual corrections and $\mathbf{b}$ ) shows the 3-parton final state at tree level.

An essential reference for the following is Appendix A, where the kinematic conventions are established. We define $x_{i}$ as the scaled energy of each parton, $x_{i}=2 E_{i} / E_{c m}$, with $x_{1} \geq x_{2} \geq x_{3}$ and $x_{1}+x_{2}+x_{3}=2$. Since we only have three partons in the final state, the kinematics leaves us with only two independent variables which we take to be $x_{1}$ and $x_{2}$.
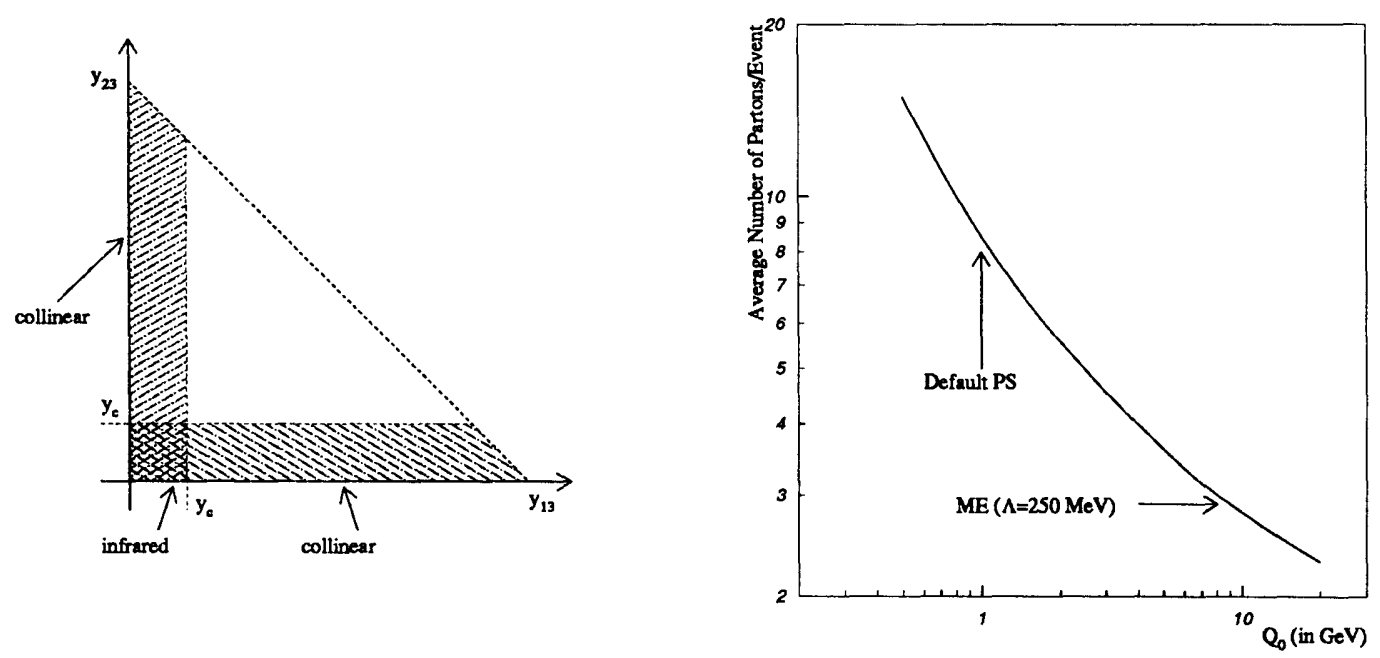

Figure 2-4: The phase space region for 3 parton final states, including the infrared and collinear regions.

Figure 2-5: Average number of partons as a function of the parton shower virtuality cutoff $Q_{0}$.

The cross section for gluon brehmsstrahlung (Figure 2-3b) is given by the well 
known result [35],

$$
\frac{d^{2} \sigma}{d x_{1} d x_{2}}=\sigma_{q} C_{F} \frac{\alpha_{s}}{2 \pi} \frac{x_{1}^{2}+x_{2}^{2}}{\left(1-x_{1}\right)\left(1-x_{2}\right)}
$$

where $\sigma_{q}$ is the lowest order $q \bar{q}$ cross section at the $Z^{0}$, given by Equation 1.2, and $C_{F}=4 / 3$ is the appropriate color factor for this color configuration. Notice that the above exhibits the explicit gluon mass singularity (for $x_{1}$ and $x_{2} \rightarrow 1$ ) since it does not have the virtual corrections added.

Eventually we will see that what allows us to make inferences about the rate of hard gluon radiation is the good (asymptotic-free justified) approximation that hadron jets correlate to the initial 'color-full' partons. With this in mind, we now shift our focus and treat Equation 2.18 as the 3-jet cross section formula (again, just to $\mathcal{O}\left(\alpha_{s}\right)$ ). But we immediately notice that for very soft gluons (say, $x_{3} \rightarrow 0$ ), we go into the 2 -jet limit $\left(x_{1}=x_{2}=1\right)$ and Equation 2.18 diverges. This is no surprise; we already noticed this gluon infrared divergence and remarked that it was cancelled with the virtual corrections of Figure 2-3a. However there is still a collinear divergence associated with the assumption of massless quarks ${ }^{7}$. Also, since we are not just interested in $\sigma_{t o t}$, we ought to have a consistent procedure to separate and define the 3 -jet events.

This last task is accomplished by dimensionally regularizing the virtual and real parts of the cross section and by partitioning the phase space of the $q \bar{q} g$ events into 'distinguishable 3-jets' and 3-jets 'indistinguishable from 2-jets'. This last category is then absorbed into the 2-jets for a particular cut-off of the 3-jet phase space. Following the convention of Appendix A, we define in terms of the invariant $y_{i j}$ the 3to-2 jet boundaries in Figure 2-4. We label the boundary ' $y_{c}$ ' or ' $y_{c u t}$ '. Remembering that $y_{23}=1-x_{1}$, we see that the shaded rectangular regions correspond to the collinear divergent parts of Equation 2.18 and that the heavily shaded square region corresponds to the infrared divergent part.

The shaded areas of the $q \bar{q} g$ phase space in Figure 2-4 correspond to the areas where two of the partons are unresolvable. In this region the 3 -jet events are indistinguishable from the 2-jet events. Rewriting Equation 2.18 in terms of the invariant measure $y$, dimensionally regularizing it, and integrating over the 2 -jet bands [26], one obtains:

$$
\begin{aligned}
\sigma_{3}^{2-j e t}\left(y \leq y_{c}\right)= & \sigma_{q}\left(\frac{4 \pi \mu^{2}}{s}\right)^{\epsilon} \frac{\alpha_{s}\left(\mu^{2}\right)}{2 \pi} C_{F} \frac{\Gamma(1-\epsilon)}{\Gamma(1-2 \epsilon)} \\
& \times\left(\frac{2}{\epsilon^{2}}+\frac{3}{\epsilon}+2 \ln ^{2} y_{c}+4 y_{c} \ln y_{c}-\frac{\pi^{2}}{3}+7\right) .
\end{aligned}
$$

In the above, $\sigma_{q}$ is given by Equation 1.2 and $\mu$ is the arbitrary mass scale introduced to keep the coupling constant dimensionless in generalized $n=4-2 \epsilon$ dimensions.

\footnotetext{
${ }^{7}$ This comes about through $\cos \theta_{q g} \rightarrow 1$ in $m_{q g}^{2}=2 E_{q} E_{g}\left(1-\cos \theta_{q g}\right)$ terms in the quark propagator of the gluon loop
} 
The subscript and superscript on the left-hand side denote that Equation 2.19 is the cross section for resolving 2-jets from a 3-parton configuration.

Doing the virtual and real integration for the 2-parton final state to $\mathcal{O}\left(\alpha_{s}\right)$ using the diagrams of Figure 2-3a and 1-1 yields,

$$
\sigma_{2}^{2-j e t}=\sigma_{q}\left(\frac{4 \pi \mu^{2}}{s}\right)^{\epsilon} \frac{\alpha_{s}\left(\mu^{2}\right)}{2 \pi} C_{F} \frac{\Gamma(1-\epsilon)}{\Gamma(1-2 \epsilon)}\left(-\frac{2}{\epsilon^{2}}-\frac{3}{\epsilon}+\frac{2 \pi^{2}}{3}-8\right)
$$

where the subscript ' 2 ' in the left hand side stresses the fact that the cross section derives from the two parton final states. Notice that Equation 2.20 has no $y_{c}$ dependence since initial two-parton states will always be resolved as 2 -jets. Now it is also clear what we meant by saying that the divergencies would cancel when all diagrams were taken into account: when adding Equations 2.19 and 2.20 to obtain the total 2 -jet cross section, the infrared and collinear pole terms $\left(1 / \epsilon^{2}\right.$ and $1 / \epsilon$ respectively) cancel in the sum. After this summation, we take the $\epsilon \rightarrow 0$ limit to recover four dimensions, and we write for the total 2-jet cross section,

$$
\begin{aligned}
\sigma_{2+3}^{2-j e t}\left(y \leq y_{c}\right) & =\sigma_{3}^{2-j e t}\left(y \leq y_{c}\right)+\sigma_{2}^{2-j e t} \\
& =\sigma_{q}\left[1+\frac{\alpha_{s}\left(\mu^{2}\right)}{2 \pi} C_{F}\left(-2 \ln ^{2} y_{c}-3 \ln y_{c}+4 y_{c} \ln y_{c}-1+\frac{\pi^{3}}{3}\right)\right]
\end{aligned}
$$

where now the 2-jet cross section depends on the resolution parameter $y_{c}$ that determines when 3 -jet events are distinguishable from 2 -jet events. We should mention that the accuracy of these calculations in the low- $y$ region has been extended recently [36] by partially resumming the next to leading logarithm terms $\left(\alpha_{s}^{n} \ln ^{m} y\right.$ terms above) in the jet rate calculations. These methods increase the measurement accuracy somewhat by reducing the renormalization scale dependence (e.g., See Table 6.9).

The above cancellation of infrared and collinear divergencies when integrating the cross section over regions of phase space where the final states are unresolvable is a specific example of the Kinoshita-Lee-Nauenberg (KLN) theorem [37]. The theorem is a general result for degenerate states ${ }^{8}$ : infrared and collinear divergencies cancel in a theory with massless fields when a summation is performed over degenerate states. This theorem holds to all orders in perturbation theory.

As a final comment, it should be pointed out that Equation 2.18 can be generalized to calculate some of the QCD observables discussed previously. However, in order to satisfy the KLN theorem and obtain sensible cross sections, these observables must respect the degeneracy of the soft and collinear partons. This means that in general good observables are linear in parton momenta (see Appendix A). Observables that satisfy these requirements are said to be infrared and collinear safe [38].

\footnotetext{
${ }^{8}$ Two massless collinear partons are degenerate because they can be treated as a single parton. The same applies to infrared degeneracy - when a parton accompanied by a very soft gluon can be combined into one parton.
} 


\section{The $\mathcal{O}\left(\alpha_{s}^{2}\right)$ Result: the ERT Calculation}

Now that we have presented an outline of the $\mathcal{O}\left(\alpha_{s}\right)$ calculation, we turn our attention to the $\mathcal{O}\left(\alpha_{s}^{2}\right)$ results. The $\mathcal{O}\left(\alpha_{s}^{2}\right)$ calculation has been performed by various groups (e.g., $[39,40])$ In our measurement we will use the calculation by Ellis, Ross, and Terrano (ERT) [39]. The ERT calculation has been done for different jet algorithms with different recombination schemes. This last issue is a technical point that we will address in Chapter 6.

In the previous section we saw that we were able to calculate the 3 -jet cross section without recurring to any renormalization technique. The reason was that we did not encounter any persistent ultraviolet divergence; all the divergencies conveniently cancelled when the appropriate accounting of diagrams was performed. In the $\mathcal{O}\left(\alpha_{s}^{2}\right)$ case however, we encounter diagrams with persistent divergencies (the loop diagrams to $\left.\mathcal{O}\left(\alpha_{s}^{2}\right)\right)$ that require a renormalization procedure. In the following, and for the rest of this work, the modified minimal subtraction renormalization scheme $(\overline{M S})$ will be used. The QCD parameter $\Lambda$ will be,

$$
\Lambda_{Q C D}=\Lambda_{\frac{n_{f}=5}{M S}},
$$

where the superscript denotes five active flavors.

The ERT result can be conveniently expressed $[34,36]$ as,

$$
\begin{aligned}
& R_{2}(y, \mu) \equiv \frac{\sigma_{2}(y)}{\sigma_{\text {tot }}}=1-A(y) \frac{\alpha_{s}(\mu)}{2 \pi}-(B(y, f)+C(y))\left(\frac{\alpha_{s}(\mu)}{2 \pi}\right)^{2} \\
& R_{3}(y, \mu) \equiv \frac{\sigma_{3}(y)}{\sigma_{t o t}}=A(y) \frac{\alpha_{s}(\mu)}{2 \pi}+B(y, f)\left(\frac{\alpha_{s}(\mu)}{2 \pi}\right)^{2} \\
& R_{4}(y, \mu) \equiv \frac{\sigma_{4}(y)}{\sigma_{\text {tot }}}=C(y)\left(\frac{\alpha_{s}(\mu)}{2 \pi}\right)^{2}
\end{aligned}
$$

where $y=y_{c}$ and where, for example, $\sigma_{2}(y)$ has the same meaning as in Equation 2.21 except that it is one order higher in $\alpha_{s}$. The total cross section in Equation 2.23, $\sigma_{\text {tot }}$, is the one given by Equation 1.1 to at least $\mathcal{O}\left(\alpha_{s}^{2}\right)$. The leading order terms $A$ and $C$ depend on $y$ only while the next-to-leading term $B$ depends on $y$ and on the renormalization scale $f=\mu^{2} / Q^{2}$,

$$
B(y, \mu)=B_{0}\left(y, Q^{2}\right)+A(y) 2 \pi \beta_{0} \ln (f) .
$$

In the above, $\beta_{0}=11-2 n_{f} / 3$ and $n_{f}$ is the number of active quark flavors.

Experimentally, the $R_{i}\left(y_{c}\right)$ above are calculated by classifying and counting events as $i$-jet events at $y=y_{c}$ and then normalizing this number to the total number of events. In practice the functions $A(y), B(y, f)$, and $C(y)$ are tabulated from Monte Carlo integrations of the cross sections [34] and then parameterized as a sum of simple polynomials [41]. 


\subsection{Non-Perturbative QCD}

Up to now we have exclusively dealt with the creation of 'hard' primary partons. So far we have been able to make predictions based on perturbation theory alone because, with typical values $Q^{2} \gg \Lambda^{2}$, we safely fall in the asymptotically free realm. However, these partons still carry the color charge. As such, two observations can be immediately made. First, since QCD does not allow colored final states, somehow the partons must arrange themselves, or their color charge, so that they end up in a colorless final state. Secondly, since partons carry color they radiate gluons. Each subsequent parton branching then has a higher value of $\alpha_{s}$ and therefore there will be copious soft gluon radiation. These two observations lead to the hadronization, or fragmentation, process. By hadronization we mean the process that turns colored partons into colorless QCD bound states. These bound states are the final state hadrons.

An exact treatment of the hadronization process requires (if at all possible) techniques that are yet unavailable. Only phenomenological and experiment-driven models attempt to simulate this complicated process. These models rely on simple dynamical pictures to reproduce a wide range of phenomena and data. Notwithstanding the unpleasantly large number of arbitrary parameters that these models have, one can say that they reproduce the data over a wide range of energy extremely well.

In the following we will briefly describe how the 'post-perturbative' part of an $e^{+} e^{-}$event is modelled. We distinguish two phases: (1) the pre-fragmentation stage, where partons develop, and (2) the hadronization stage where partons coalesce into stable hadrons. It is important to remember that the following is just a tool that allows us to make a measurement in the perturbative regime. In this analysis we are not interested in the details of the fragmentation mechanism; we will in fact try to unfold these effects.

An excellent review of many of the currently available QCD generators and their methodology can be found in [42]. Detailed experimental studies and comparisons at the $Z^{0}$ energy of some of these generators can be found in $[43,44]$.

\subsubsection{Pre-fragmentation}

Parton production is generally carried out by one of two ways: by an exact matrix element method or by an iterative leading logarithm approximation method.

\section{Matrix Element}

The 'matrix element' (ME) method is an exact implementation of the $\mathcal{O}\left(\alpha_{s}^{2}\right)$ calculation discussed in the previous section. In this method, the cross section is sampled by the Monte Carlo method and, at most, four partons are produced. The partons are then hadronized. 
The main parameters for this procedure are $y_{c}$ (see previous section) and $\Lambda_{\overline{M S}}$. A parton invariant mass cut of $y_{c}>0.01$ insures that the cross sections are physical (positive). The parameter $\Lambda_{\overline{M S}}$ controls the value of $\alpha_{s}$ through the second order expression 2.15 with $Q^{2}=M_{Z}^{2}$. An alternative scheme uses $Q^{2} \ll M_{Z}^{2}$ with a lower value of $\Lambda_{\overline{M S}}$ in order to get a better prediction of the 4-jet rate at low-y. We will discuss these optimized scales in Chapter 6.

In this analysis we will use the matrix element implementation of the JETSET [45] Monte Carlo, version 6.3.

\section{Parton Showers}

Parton showers (PS) [42] are a very different approach to parton generation. In this method, partons are generated in an iterative fashion by the leading logarithm approximation (LLA). In the LLA, only the leading terms in the perturbative expansion are kept, limiting the parton splittings to $1 \rightarrow 2$ partons. This method is an attempt to approximate the 'real picture' by generating more and softer partons than in the ME approach. Thus, effectively, higher order terms are implicitly approximated and observables calculated only to leading order in the ME approach (like the 4-jet rate) are better reproduced (we will discuss this point in Chapter 6). The implementation of this approximation is via a set of Altarelli-Parisi [46] parton splitting functions that incorporate the allowed splittings to leading order: $g \rightarrow g g, g \rightarrow q \bar{q}$, and $q \rightarrow q g$.

A short description of the procedure follows. Using $t \equiv \ln Q^{2} / \Lambda^{2}$, the AltarelliParisi equations for parton splittings are given by,

$$
\frac{d \mathcal{P}_{a \rightarrow b c}}{d t}=\int d z \frac{\alpha_{s}\left(Q^{2}\right)}{2 \pi} P_{a \rightarrow b c}(z)
$$

where $\mathcal{P}_{a \rightarrow b c}$ is the probability that a branching will occur in the $Q^{2}$ interval $d t=$ $d Q^{2} / Q^{2}$, and where $z=\left(E+p_{z}\right)_{b} /\left(E+p_{z}\right)_{a}$. The $P$ splitting functions are given by [42]:

$$
\begin{aligned}
& P_{q \rightarrow q g}(z)=\frac{4}{3}\left(\frac{1+z^{2}}{1-z}\right) \\
& P_{g \rightarrow g g}(z)=3 \frac{(1-z(1-z))^{2}}{z(1-z)} \\
& P_{g \rightarrow q \bar{q}}(z)=\frac{n_{f}}{2}\left(z^{2}+(1-z)^{2}\right) .
\end{aligned}
$$

The probability that a parton starting with the virtuality $t$ will reach $t_{\min }$ without undergoing any splittings is then given by,

$$
S_{a}(t)=\exp \left(-\int_{t_{\min }}^{t} d t^{\prime} \frac{d \mathcal{P}_{a \rightarrow b c}}{d t^{\prime}}\right)=\exp \left(-\int_{t_{\min }}^{t} d t^{\prime} \int_{z_{\min }\left(t^{\prime}\right)}^{z_{\max }\left(t^{\prime}\right)} d z \frac{\alpha_{s}\left(Q^{2}\right)}{2 \pi} P_{a \rightarrow b c}(z)\right),
$$


where $t_{\min } \equiv Q_{0}^{2} / \Lambda^{2}, Q_{0}$ is called the shower virtuality cut-off, and the above factor $S_{a}$ is termed the Sudakov factor. The number of partons left over after this evolution depends critically in this $Q_{0}$ cut-off. Figure 2-5 shows this dependence for the JETSET 6.3 Parton Shower (PS) Monte Carlo. Note that the $\Lambda$ used in the LLA approximation is not the $\Lambda_{\overline{M S}}$ from the previous sections; we now deal with an effective $\Lambda_{\text {eff }}=\Lambda_{L L A}$ that just characterizes the splittings.

This whole procedure is straightforward to implement in a Monte Carlo environment. Notice that there are two parameters intrinsic to parton showers: $Q_{0}$ and $\Lambda_{e f f}$. A schematic representation contrasting matrix element and parton shower approaches is presented in Figure 2-6.

Matrix Element Models

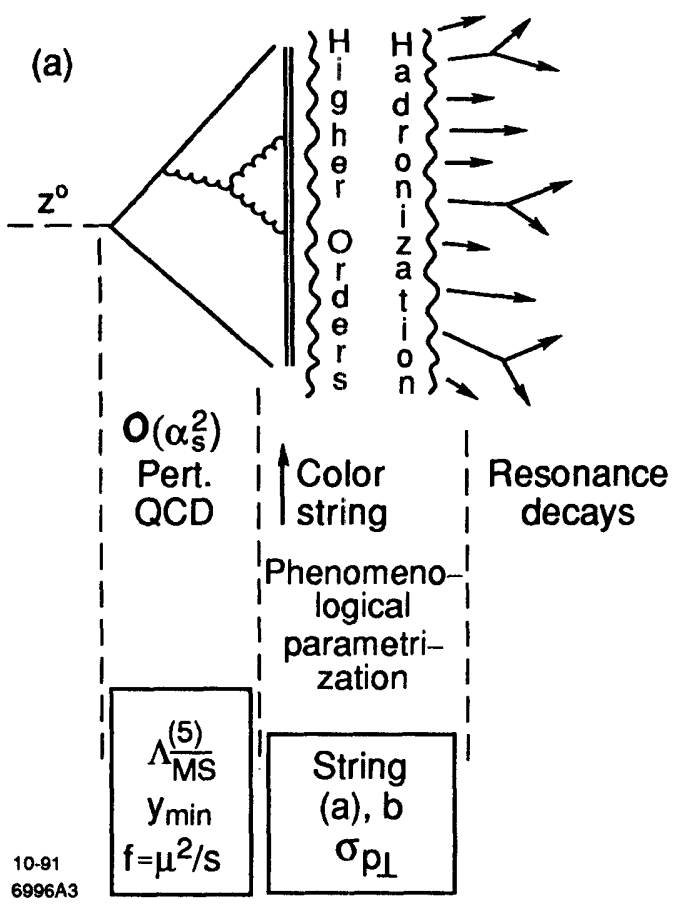

\section{Parton Shower Models}

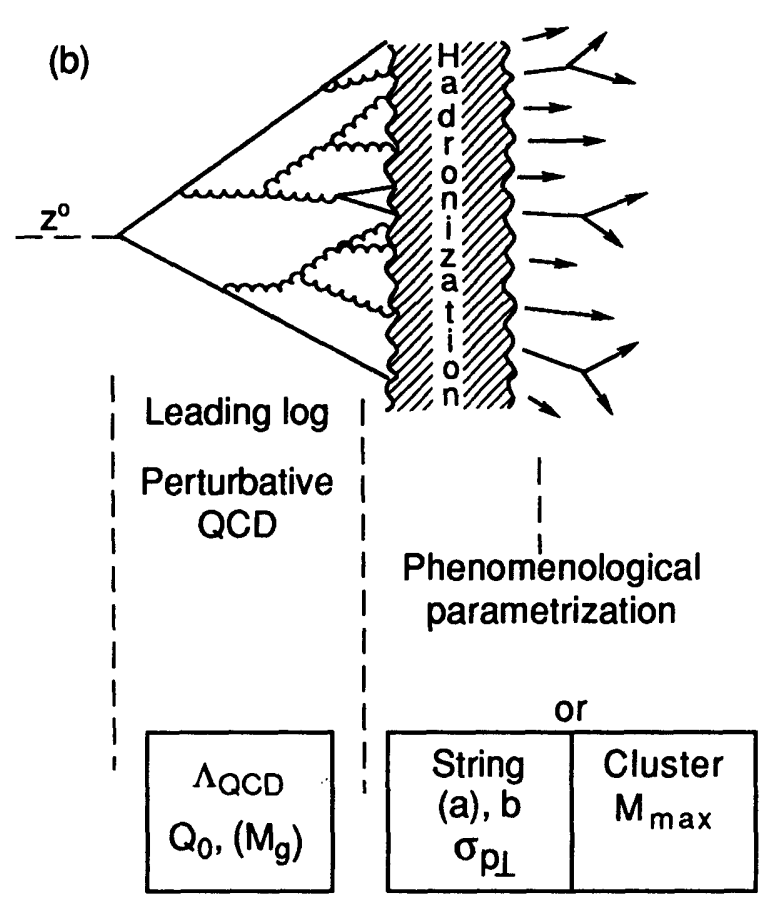

Figure 2-6: Schematic of Shower and Matrix Element Models [47].

\subsubsection{Hadronization}

Once the colored partons stop evolving (at least in the PS picture), they decay into hadronic final states. Whereas the prefragmentation part can be treated in the perturbative approximation, the hadronization mechanism is purely non-perturbative.

If we assign a characteristic transverse momentum $p_{t}$ (with respect to parent quark, i.e., jet direction) to the final state particles in an $e^{+} e^{-}$event then we can see how hadronization effects depend on energy, at least for jet quantities. Consider events at the energy $E$, with average multiplicity $n \approx a+b \ln E$. The average particle 
momenta are $p \approx E / n$ and the mean half angle of a cone about the primary quark direction is then [26]:

$$
\delta \approx \frac{p_{t}}{p} \approx \frac{p_{t} n}{E} .
$$

From the above we can see that the jet cone size goes like $\approx \ln E / E$. Therefore, at higher center of mass energies, our lack of knowledge about hadronization becomes less of an issue since there is less overlap between the jet cones.

In our analysis, we will use two of the most successful hadronization models available: the Lund string model $[48,49]$. and the HERWIG [50] cluster fragmentation model. These models, along with others, have been amply tested (e.g., $[43,44,51,52])$ and tuned at various energies in $e^{+} e^{-}$annihilation. We take advantage of this and use the parameters obtained at LEP by the L3 experiment (for the Lund model) and presented in Table 2.1 as our default parameters. The HERWIG Monte Carlo was used as a cross check to JETSET; the HERWIG parameters used are the default values provided by the program (version 5.4).

We now provide a brief description of each model used.

\section{Lund String Model}

The Lund string fragmentation model can be coupled to either a matrix element or a parton shower treatment of the primary partons. The basic dynamical picture in this model is the following [48]: when two colored quarks separate, a color flux tube is stretched between them. This flux tube is modelled by a relativistic massless (one dimensional) string with a string constant of $\kappa \approx 1 \mathrm{GeV} / \mathrm{fm}$. A linear confinement force is thus included by construction. In the string picture, the ends of the string correspond to a quark and an anti-quark, while gluons are represented by excitations or "kinks" in the string.

While the string evolves, it can break up into two color $q \bar{q}$ singlets; this rate of break-up is calculated from a semiclassical model of fermion pair tunnelling from the vacuum. The energy carried off by the daughter singlets at each break-up is well determined by the 'left-right' symmetry principle. This principle states that the final particle distributions should be blind to the order in which causally disconnected break-ups happen. Using $z$ as in the previous section, this symmetry restricts the fragmentation function to the form,

$$
f(z)=\frac{N}{z}(1-z)^{a} \exp -\frac{b m_{t}^{2}}{z},
$$

where $m_{t}$ is the transverse mass $\left(m_{t}^{2}=m^{2}+p_{t}^{2}\right)$ of the final state and $a$ and $b$ are arbitrary constants to be determined from experiment. This iterative break-up of the string into hadrons stops when the mass of the string fragments reach the hadronic scale. An additional parameter, $\sigma_{q}$, establishes the transverse momentum distributions for partons. Table 2.1 summarizes the parameters used in the Lund 


\begin{tabular}{||c|c|c|c|c||}
\hline \hline \multicolumn{5}{|c||}{ LUND Parameters } \\
\hline & $\Lambda_{Q C D}$ & $a$ & $b$ & $\sigma_{q}$ \\
\hline \hline TASSO [52,53] & 0.260 & 0.18 & 0.34 & 0.39 \\
L3 [44] & $0.300 \pm 0.03$ & 0.5 & $0.76 \pm 0.08$ & $0.39 \pm 0.03$ \\
\hline \hline
\end{tabular}

Table 2.1: LUND fragmentation parameters used in this analysis. The L3 tuned parameters were used and the TASSO parameters (tuned at $35 \mathrm{GeV}$ ) were used as a crosscheck. The TASSO numbers are known to $\approx 10 \%$ [53].

Monte Carlo.

The Lund implementation of the string model (JETSET) includes many effects not described here (baryon production, Bose-Einstein effects, etc); for more details refer to [45] and references therein.

\section{HERWIG Model}

The HERWIG Monte Carlo [50], as applied to $e^{+} e^{-}$annihilation, is a general QCD generator that uses parton showers and a cluster hadronization model. In HERWIG's implementation of parton showers, the parton-parton correlations due to spin and coherent emission of soft gluons are taken into account. The treatment of soft gluons is improved (when compared to Lund's PS) via an extension of the leading logarithm approximation in which infrared logarithms are included. The shower evolution (probability-wise) in HERWIG's scheme is very similar to Lund's; the same Sudakov factors (Equation 2.27) determine the branching probabilities.

The hadronization mechanism in HERWIG is based on a color cluster model. In the leading log approximation in HERWIG, a parton of color $j$ that finds itself close in phase space to a parton of color $\bar{j}$ is combined into a cluster. 'Close' in this context means that the quark and associated anti-quark have an invariant mass $\approx 1-2 \mathrm{GeV}$. Gluons are produced and decay into quarks. Thus, at the end of the chain, and before clustering, only partons with a single color index remain. The final clusters are characterized only by their four momenta and by their flavor composition. In the final step, the clusters decay (respecting flavor conservation) into hadrons via a one or two body mechanism, depending on the cluster mass and availability of final states.

The HERWIG Monte Carlo is described in much more detail in [42] and [50]. 


\section{Chapter 3}

\section{Experimental Apparatus}

\subsection{Introduction}

The data used in this analysis were collected at the SLC/SLD facility at the Stanford Linear Accelerator Center (SLAC) located in Stanford, California. The Stanford Linear Collider [54] (SLC) was a unique $e^{+} e^{-}$linear accelerator that took advantage of SLAC's existing $50 \mathrm{GeV}$ electron accelerator. The SLD was a state-of-the-art full coverage multi-purpose particle detector placed at the SLC's only interaction region. In the following sections we will describe in some detail the hardware that made our measurement possible.

\subsection{The Stanford Linear Collider}

The SLC was a single-pass electron-positron collider located at one end of SLAC's Linear Accelerator (LINAC). As a single-pass machine, the SLC did not enjoy the inherent stability that is a requirement for maintaining beam current in a storage ring. We will see in Chapter 5 that the machine performance, in terms of luminosity, was not at the expected design levels during the 1992 run. In addition, the run conditions were considerably noisy. Nevertheless, the SLC had some important advantages over comparable machines: it was able to deliver beams of longitudinally polarized electrons and it provided a spatially precise interaction point (from here on, 'IP').

\subsubsection{The Polarized SLC}

The SLC [55] consisted of two main sections: a 2-mile long linear accelerator and a set of arches that met at one interaction point. The accelerator was housed in an underground tunnel below the 'klystron gallery', a 2-mile building full of power supplies, LINAC control equipment, and RF equipment. Each arch was roughly a half mile long and contained beam steering optics, muon spoiler magnets, and the 


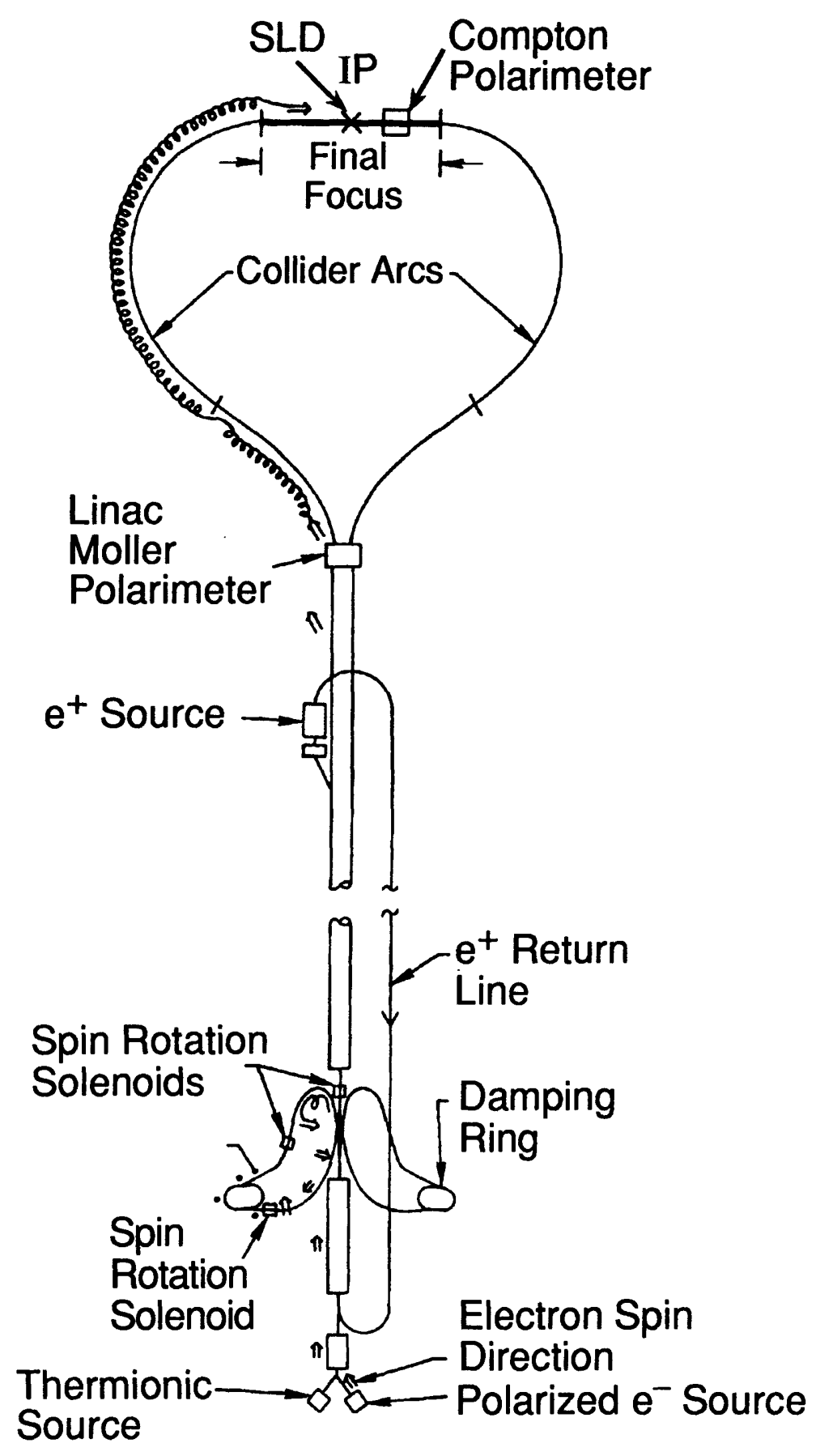

292

7220A5

Figure 3-1: The Polarized SLC. The double arrows denote the relative orientation of the spin vector. The energy at the end of the LINAC was $46.7 \mathrm{GeV}$; the IP beam energy was $45.8 \mathrm{GeV}$. 
final focus system.

Using Figure 3-1 as a reference we will briefly follow the path, from acceleration to annihilation, of a typical electron in the SLC. During the 1992 run, longitudinally polarized electrons were produced by photo-emission from a gallium arsenide photocatode at the very beginning of the LINAC structure (bottom of the picture). The polarized electron emissions from the cathode were induced by an incident circularly polarized laser beam $(\lambda=715 \mathrm{~nm})$. A dual source was actually available - a thermionic source was available to run in parallel with the photocathode in case the latter failed. The polarization at the source was $\approx 28 \%$ [56].

The SLC operated with a machine cycle of $120 \mathrm{~Hz}$. Two electron pulses were produced during each cycle; each had a temporal width of 2 ns and was separated from the other by $61 \mathrm{~ns}$. Each pulse contained $\approx 6(10)^{10}$ electrons when leaving the source. After the damping ring, combined transmission losses reduced this number by $50 \%$. After they left the source, both pulses were accelerated to $1.16 \mathrm{GeV}$ before entering the North damping ring (left damping ring in Figure 3-1). The pulses were then extracted from the damping rings and the leading pulse accelerated to 46.7 $\mathrm{GeV}$ before entering the SLC north arc. The trailing pulse was accelerated to 30 $\mathrm{GeV}$, extracted from the LINAC, and guided to hit a target in order to produce the positrons. The positrons were then transported to the beginning of the accelerating cycle where they underwent the same acceleration as the electrons from the leading pulse except that they then went into the South damping ring.

Since there were no accelerating structures in the SLC arches, the pulses had to be accelerated to $46.7 \mathrm{GeV}$ (instead of $M_{Z^{\circ}} / 2$ ) in the LINAC in order to compensate the synchrotron energy losses due to bending. In order to achieve the $90^{\circ}$ turn in the particle path necessary for a collision, each arch contained a series of 23 achromats (a set of multifunction magnets) that guided the particle bunches through the beampipe. Things were further complicated by the fact that the SLC tunnel did not lie on a plane; instead it followed the terrain.

Before entering the SLD the beams went through the Final Focus system at the end of the arches. The Final Focus were a set of superconducting magnets that squeezed the beam size down to $\sigma_{x, y} \approx 2-3 \mu m$ in preparation for the final collision. The beams then collided at the interaction point. In a linear collider, the luminosity can be expressed as [57],

$$
\mathcal{L}=\frac{n_{1} n_{2} f_{c}}{4 \pi \sigma_{x} \sigma_{y}}
$$

where $n_{1}$ and $n_{2}$ are the number of particles per bunch, $f_{c}$ is the collision rate, and $4 \pi \sigma_{x} \sigma_{y}$ is the effective beam-beam crossing area in a Gaussian beam. Typical SLC luminosities during the 1992 run [58] were $\mathcal{L} \approx 2.5(10)^{29} \mathrm{~cm}^{-2} \mathrm{~s}^{-1}$ with a machine uptime $\approx 60-70 \%$. We will discuss the direct measurement of $\mathcal{L}$ in Section 5.1.1.

After the electron bunch passed the interaction point, the beam polarization was measured using a Compton polarimeter [59] in the South arch. The nominal electron polarization measured at the Compton polarimeter was $22.4 \pm 0.7 \%$ [59]. Before pro- 
ceeding to the beam dumps, the energy of each beam was measured in a spectrometer.

In order to reduce the beam-induced backgrounds (which will be discussed in Section 5.1.2) at the SLD, toroidal magnets were installed in both the North and South arches. These magnets ("muon-spoilers") were designed to alternatively focus and defocus the background muons that travelled parallel to the beam pipe so that they would either be absorbed in the tunnel walls or be deflected away from the detector.

\subsubsection{Beam Energy Measurement}

The beam energies in the SLC were measured by a Wire Imaging Synchrotron Radiation Detector (WISRD) [60] present in each arch. These detectors were essentially deflection spectrometers placed at the end of the beam transport system. They were located after the interaction point and before the beam dump so that there were no noticeable bending losses. Before reaching the WISRD, each beam went through a series of three dipole magnets in a split-beam configuration. The first magnet (refer to Fig. 3-2) induced a horizontal spray of synchrotron radiation that provided a reference pedestal for the bending downstream. The second magnet was a precisely calibrated analyzing dipole which bent the beam 15 meters upstream of the WISRD. The third magnet provided another horizontal stripe of synchrotron radiation. The position of this last stripe was compared to the position of the first one to measure a deflection. The beam energy was then calculated as,

$$
E_{\text {beam }}=\frac{c}{\theta} \int|\vec{B} \times \overrightarrow{d l}|
$$

where $\theta$ is the measured beam deflection, $\vec{B}$ is the magnetic field in the analysis magnet, and $\overrightarrow{d l}$ is the path length along the beam. The synchrotron radiation was detected by Compton scattering of the electrons in two screens of copper wires. The measured mean center-of-mass energy for the 1992 run was $91.55 \pm 0.04 \mathrm{GeV}$ [61].

\subsection{The Stanford Large Detector}

The Stanford Large Detector (SLD) was conceived [62] as a high precision general purpose detector. The aim in the design was to provide a $4 \pi$ coverage detector suitable for the center-of-mass energies of $\approx 100 \mathrm{GeV}$ of the SLAC linear collider (SLC). The main physics motivation at that time (in 1984) was to study the then [4] newly discovered $Z^{0}$ particle region. To this end the SLD was designed with the capabilities to perform new particle searches and precision electroweak measurements. The detector was divided in 6 distinct detector subsytems with complementary functions and had a generalized Barrel-Endcap geometry. To provide for precision tracking of charged particles, the SLD had a CCD-based vertex detector and a barrel-endcap drift chamber system. A lead-liquid argon barrel-endcap calorimeter provided the energy 


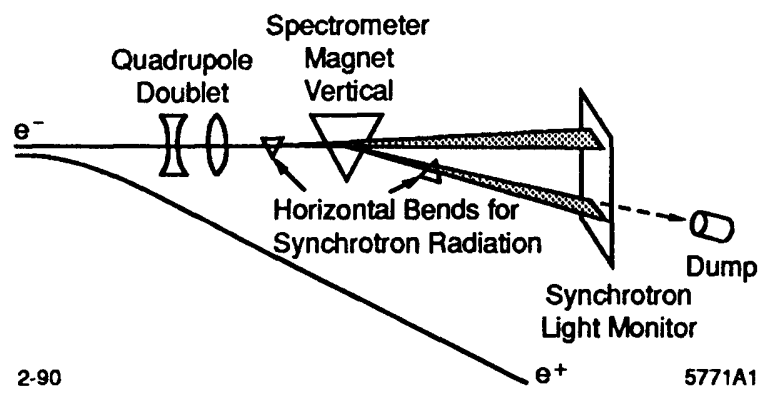

Figure 3-2: Schematic of the SLC energy spectrometer. There was one spectrometer in each arch just before the beam dump. The energy at the end of the LINAC was 46.7 $\mathrm{GeV}$; the beam energy at the IP was $45.8 \mathrm{GeV}$.

measurement for both neutral and charged particles. The Warm Iron Calorimeter, based on Iarocci tube technology, served as a tail-catcher for the LAC and provided good muon identification; the WIC also functioned as a magnetic flux return. A coil between the WIC and the LAC provided a 0.6 Tesla solenoidal magnetic field for momentum measurement. The luminosity at the SLD interaction point was measured by a silicon-based calorimeter close to the beam-pipe. Finally, a Cherenkov Ring Imaging system provided particle identification information. Figure 3-3 provides a quadrant view of SLD showing the relative configuration of each detector subsystem.

A brief description of each of the subsystems follows. A more detailed description of the calorimeter system will be provided since it was the relevant subsystem for this analysis.

\subsubsection{The Vertex Detector}

The SLD vertex detector (VXD) was a high-precision tracking device very close to the SLC beam-pipe. Such devices are very helpful in separating tracks from secondary vertices (decay points from heavy flavor decays) from tracks originating in the primary vertex (interaction point). In addition, by combining the precision tracking in the VXD with the drift chamber measurement, the overall momentum measurement was improved.

The VXD was the first detector [63] to implement the large-scale use of charge coupled devices (CCDs). It consisted of $480 \mathrm{CCDs}$ each with a matrix arrangement of $400 \times 600$ pixels. Each pixel was $22 \times 22 \mu \mathrm{m}^{2}$ in size, yielding an effective position resolution of $\sigma_{x, y} \approx 5 \mu \mathrm{m}$. The CCDs were arranged in an overlapping fashion into four layers of concentric 8-CCD ladder elements. There were a total of 60 such ladders for a grand total of 120 Megapixels. A track exiting the VXD would traverse, on average, a total of $5.8 \%$ of a radiation length (c.f., Figure 3-11). The SLD beampipe had a 


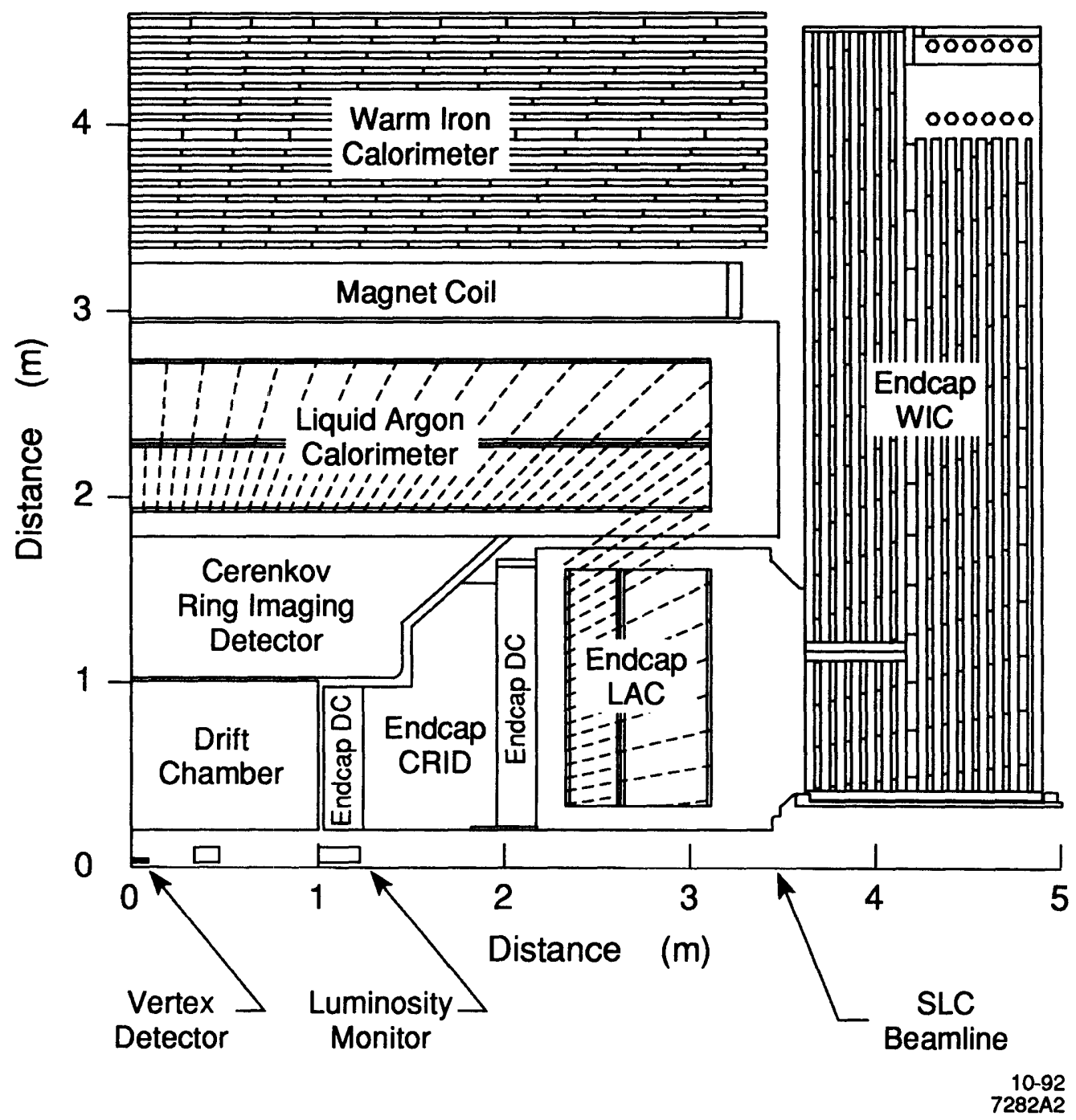

Figure 3-3: A quadrant view of SLD. The lower left corner corresponds to the SLD interaction point (IP). Notice the projective tower geometry of the calorimeter system. 


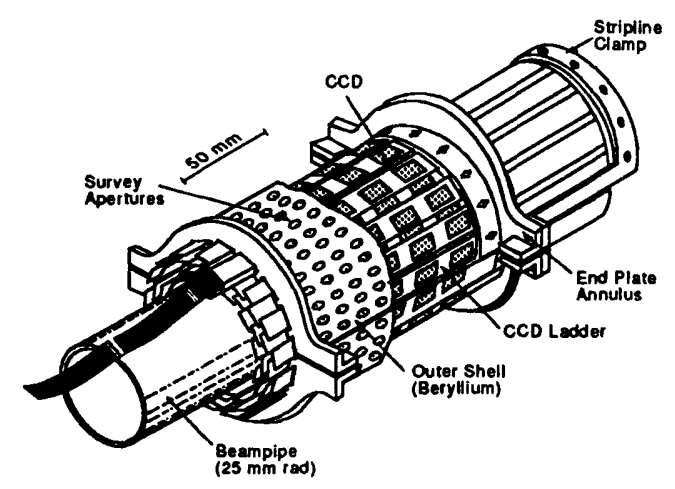

Figure 3-4: The SLD vertex detector.

radius of $2.55 \mathrm{~cm}$; the first VXD layer was at a radius of $2.95 \mathrm{~cm}$ and the last layer was at $4.15 \mathrm{~cm}$. The detector itself was mounted inside the central drift chamber on the R20 module. The R20 module refers to the assembly where the detector components at a radius of $R \leq 20 \mathrm{~cm}$ and $|z| \leq 100 \mathrm{~cm}$ were mounted. The R20 module included 2 meters of beryllium beampipe, various synchrotron radiation masks, the medium angle silicon calorimeter (MASiC), and the VXD with its associated electronics. The VXD acceptance roughly corresponded to the acceptance of the central drift chamber (CDC).

\subsubsection{The Luminosity Monitor}

The luminosity monitor $[64,65]$ in SLD consisted of a luminosity monitor and small angle tagger (LMSAT) and a medium angle silicon calorimeter (MASiC). This system was essentially a very low angle and high precision electromagnetic calorimeter. The LMSAT/MASiC system was a silicon sampling calorimeter with a tungsten radiator and a $1.44 \%$ sampling fraction. By tagging forward ${ }^{1}$ (i.e. small angle with respect to beam-pipe) $e^{+} e^{-}$final states a precise measurement of the luminosity was made. In Section 5.1.1 we will discuss this in more detail.

Figure 3-5 shows a side view of the LMSAT/MASiC assembly. The LMSAT provided an angular coverage of 23 to 68 milliradians from the SLD beampipe at a distance of $100 \mathrm{~cm}$ from the interaction point. The total depth of the calorimeter was $21 X_{0}$ providing a shower containment of $\geq 99.5 \%$. The LMSAT consisted of 23 radiator plates, each approximately one radiation length deep, instrumented with silicon detectors between the plate gaps. The MASiC covered the area of 68 to 190 milliradians and sat $31 \mathrm{~cm}$ away from the IP. Each layer was transversely segmented in a projective tower geometry. The polar segmentation was 9 milliradians and the

\footnotetext{
${ }^{1}$ The t-channel part of $e^{+} e^{-} \rightarrow e^{+} e^{-}$is essentially QED dominated and it peaks in the forward region.
} 
segmentation in $\phi$ was 11.25 degrees. This fine segmentation was important since no tracking was available at these low angles to determine electron positions. The LMSAT was mounted on the superconducting final focus triplet magnet and the MASiC was mounted on the R20 module discussed in the previous section. The expected energy resolution for a $50 \mathrm{GeV}$ electron was $\sigma_{E} / E \approx 3.0 \%$.

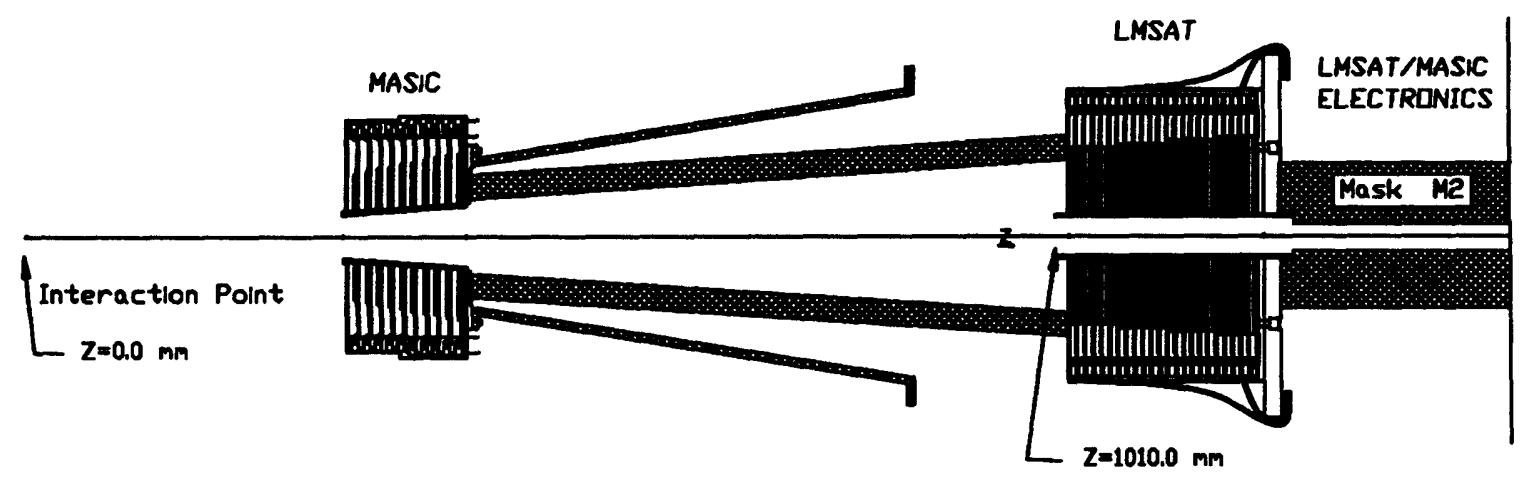

Flgure 1. LUminosity Monitors for SLD

Figure 3-5: Side view of LMSAT/MASiC Assembly.

\subsubsection{The Drift Chamber System}

The SLD drift chamber system [62] provided momentum and position measurements of charged particles. It consisted of five drift chamber subsystems: the central barrel region (CDC) and four endcap regions (EDC). The central drift chamber was a cylindrical chamber with an inner radius of $20 \mathrm{~cm}$ and an outer radius of $100 \mathrm{~cm}$. It was 2 meters long and rested inside of the CRID. The chamber was made of a large set of wires under tension supported by the chamber endplates. Wires were arranged in 10 superlayers. Each superlayer was made up of cells with 8 sense wires, 24 guard wires, and 27 field wires each. Each superlayer orientation alternated between axial and stereo in the configuration: AUVAUVAUVA, where ' $\mathrm{A}$ ' stands for axial layer and 'UV' are stereo layers. The wires establish electrostatic fields that cause electrons from track induced ionization to drift and undergo amplification in the gas through the avalanche mechanism. Charge division was also used for pattern recognition.

The endcap drift chambers complemented the CDC measurement in the forward region $\left(\theta>45^{\circ}\right)$. There were two sets of endcap detectors, inner and outer, per endcap. The endcaps were placed at $z= \pm 1.2 \mathrm{~m}$ and $z= \pm 2.0 \mathrm{~m}$. Each of the four endcap chambers had three superlayers with a relative rotation of $60^{\circ}$. The approximate measured momentum resolution [66] for the CDC was $\left(\frac{\sigma}{p}\right)^{2}=(0.01)^{2} / p^{2}+(0.007)^{2}$ with a local spatial resolution of 120 microns. 


\section{The Magnet and Flux-return}

The SLD magnet was a normal water-cooled aluminum magnet that generated a solenoidal magnetic field of 0.60 Tesla for the momentum measurement. The coil itself was $0.6 \lambda_{\text {int }}$ deep and rested inside of the WIC barrel. The WIC barrel and endcap steel housing functioned as a flux return for the magnet.

\subsubsection{The Cherenkov Ring Imaging Device}

The purpose of the Cherenkov Ring Imaging Device [62] (CRID) was to provide good particle identification over a wide range of momenta. In order to achieve good $\pi / K / p$ separation over the required momentum range, the CRID was designed as a hybrid gas-liquid Cherenkov system. Coverage of the barrel and endcap regions were provided by separate systems.

A charged particle that entered the CRID encountered a liquid radiator $\left(C_{6} F_{14}\right)$ with an index of refraction of $n=1.277$. Cherenkov photons from the liquid radiator were proximity-focused in a photon detector between the liquid and the gas radiator. The gas radiator $\left(C_{5} F_{12}, n=1.002\right)$ had a series of mirrors at the end that would precisely back-focus the photons into the photon detector. The photon detector consisted of a series of drift boxes filled with a photo-ionizing gas (TMAE) and a set of wires in an electric field for the detection of the photo-electrons.

\subsubsection{The Calorimeter System}

The SLD calorimeter system was a hybrid lead-liquid argon and iron-gas sampling calorimeter [62]. Both calorimeter components had a barrel-endcap geometry and coverage that extended very close to the beampipe. All of the calorimeter subsystems followed the same projective tower segmentation. The projective geometry simplified both electromagnetic and hadronic shower reconstruction by following the particle paths from the interaction point. The calorimeter data acquisition system read out all of the calorimeter subsystems simultaneously and in a transparent fashion. The LUM/MASiC was formally part of the calorimeter system; it has already been described in Section 3.3.2. The Liquid Argon Calorimeter (LAC) also provided an energy-based trigger to be described in detail in Section 5.3.

In the following we will briefly describe the calorimeter hardware. In the next chapter we will study the performance and the calibration of the calorimeter system in more detail. For reference, Figure 3-3 shows the relative placement of the various calorimeter subsystems. Table 3.1 summarizes the material specifications of the calorimeter and Figure 3-11 gives a more detailed accounting of the amount of material throughout the detector as a function of the polar angle. 


\section{Calorimetry}

Here we will briefly summarize the terms and ideas central to calorimetry. "Calorimetry", in our context, refers to the destructive measurement of a particle's (or group of particles') energy. Typically, a particle enters a calorimeter and interacts. The interaction can be either electromagnetic (generally initiated by photons or electrons) or hadronic (initiated by inelastic scattering of hadrons). These interactions, if sufficiently high in energy, develop into showers - literally a stream of either produced or 'knocked-out' particles. Electromagnetic showers are a function of $X_{0}$ and hadronic showers depend on $\lambda_{\text {int }}$. Since the only participants in electromagnetic showers are photons and electrons, electromagnetic showers are easily parameterized. Hadronic showers are more complicated because they suffer a myriad of different interactions and energy loss mechanisms. In the simplest of models, when one considers hadronic shower development involving pions, then a purely electromagnetic component to the hadronic shower creeps in (the $\pi^{0}$ ). This component causes large fluctuations, especially if it appears early in the shower.

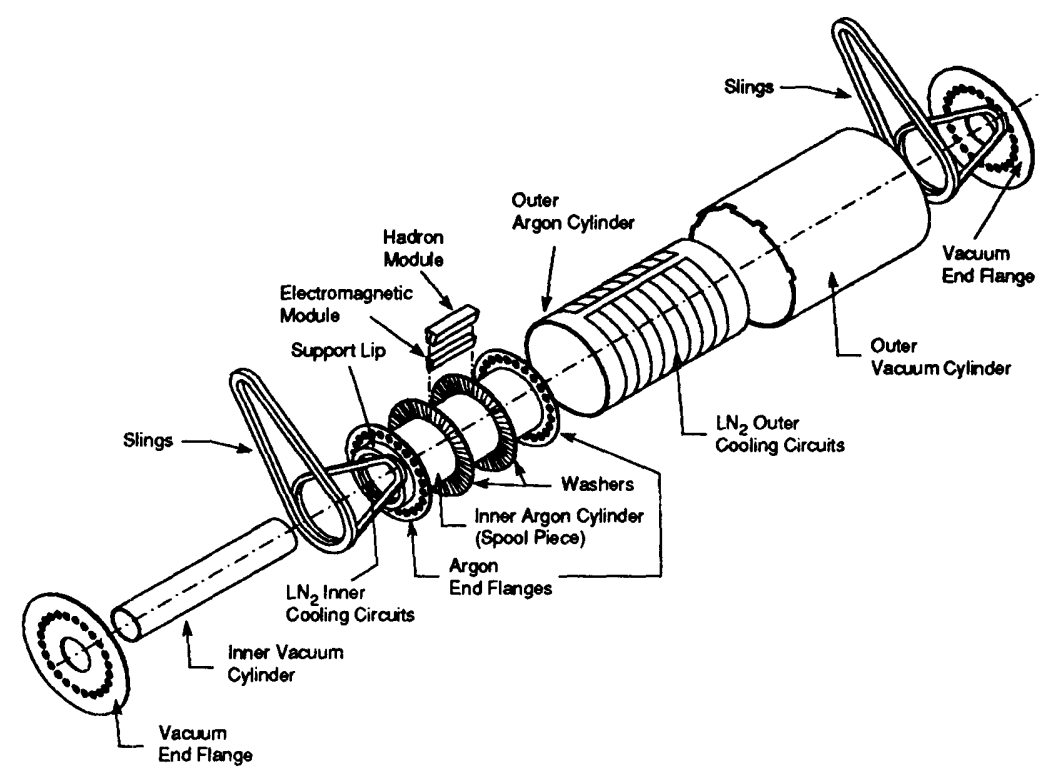

Figure 3-6: Exploded view of the LAC barrel.

A calorimeter generally works by detecting the remnants of interactions by measuring ionization signatures. Sampling calorimeters are most commonly used in high energy experiments to minimize the size, instrumentation, and cost of the detector system. They consist of a 'sandwich' of active material (where ionization is detected) and passive material (high- $Z$ absorber material to catalyze shower development) in carefully chosen ratios. The fraction of energy lost by a mip particle (we will define mip in Chapter 4) in the active part of the calorimeter is called the sampling fraction:

$$
F_{\text {sampling }}=\frac{(d E / d X)_{\text {active }}}{(d E / d X)_{\text {active }}+(d E / d X)_{a b s o b e r}} .
$$




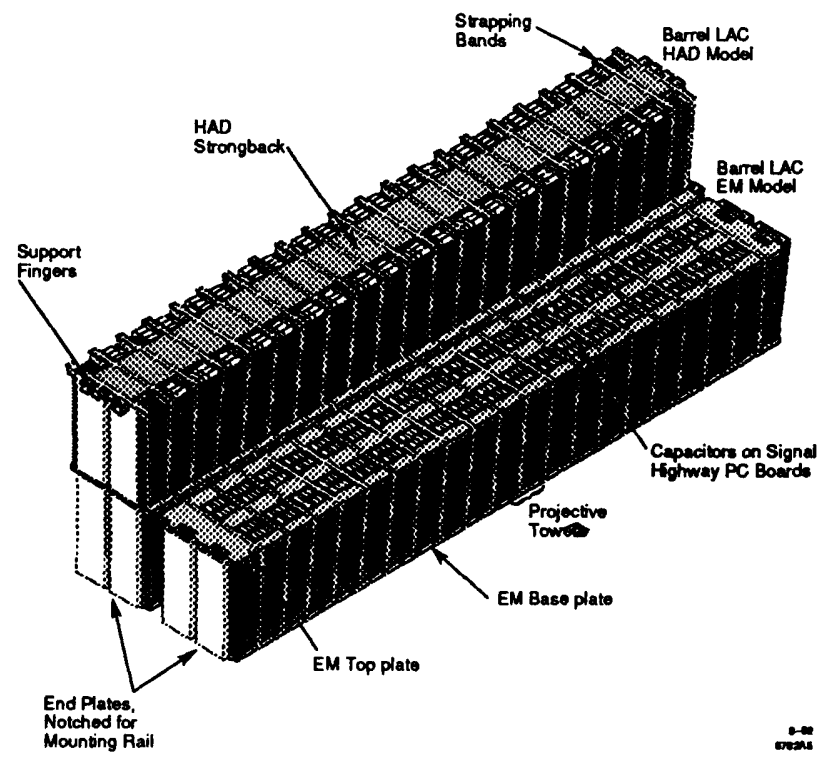

Figure 3-7: A LAC barrel module.

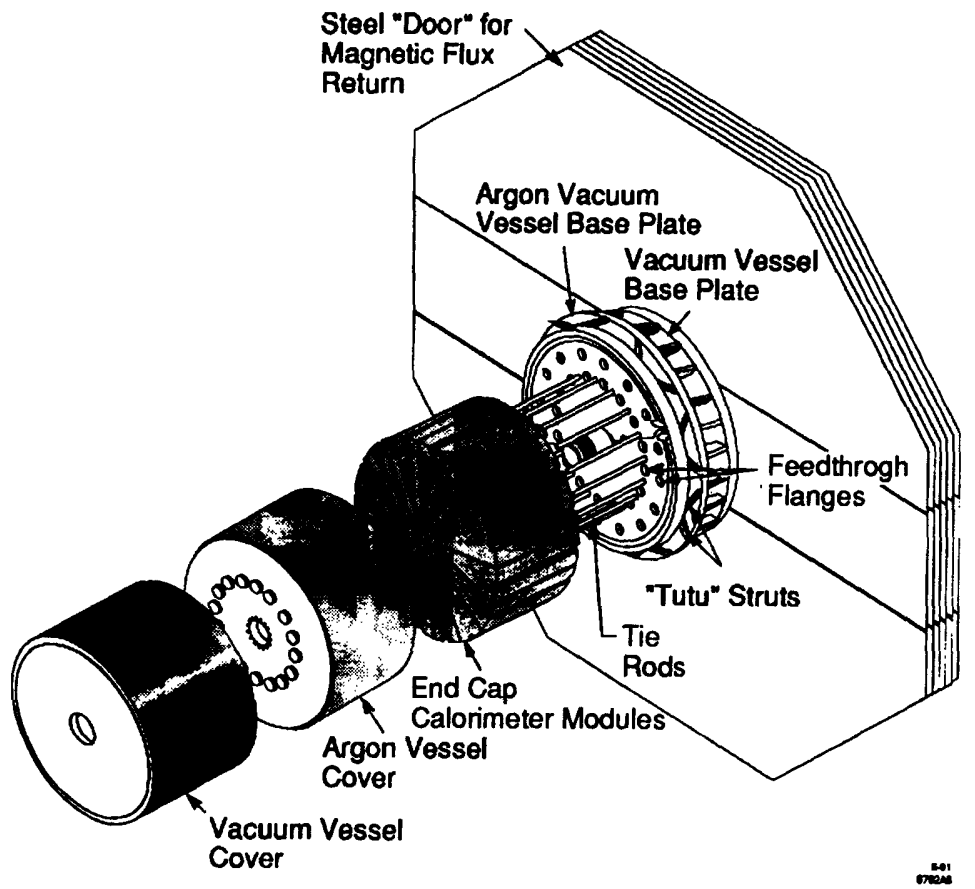

Figure 3-8: Exploded view of the LAC endcap. 


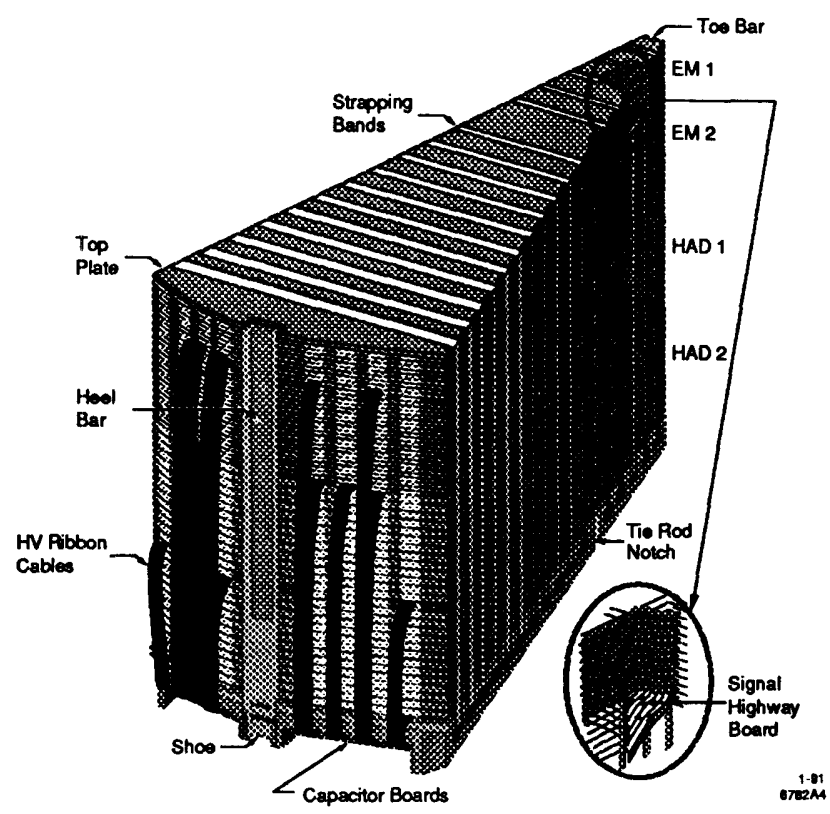

Figure 3-9: A LAC endcap module.

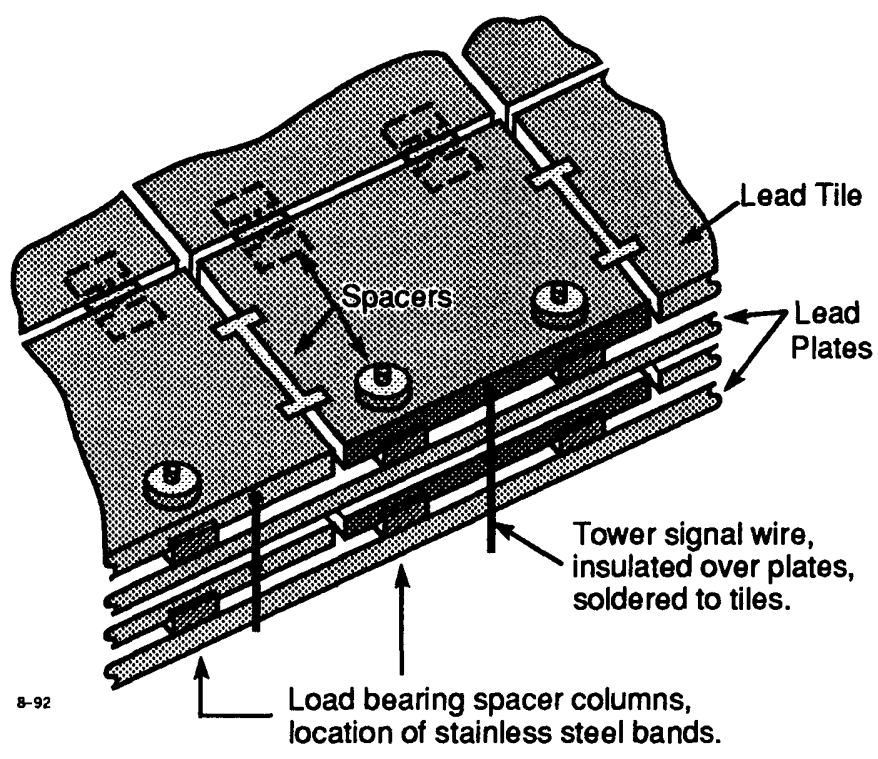

Figure 3-10: Detail of the LAC cell geometry.

Hadronic showers also have an invisible energy component [67] due to neutrinos, muons, nuclear binding energy losses, and other such processes that accounts for roughly $40 \%$ of the total incident energy. The ratio of electromagnetic to hadronic response is therefore generally not 1.0 . A calorimeter with equalized response is said to be compensating. A non-compensating calorimeter will suffer from energy nonlinearities and resolution degradation due to the fluctuations in the electromagnetic component of the hadronic shower. 


\section{The Liquid Argon Calorimeter}

The idea of using liquid argon sampling calorimeters was first introduced in 1974 [68] and has, since then, been extensively studied $[69,70,71,72,73,62,74]$. The main advantage of liquid argon calorimetry is that the medium, having unity gain ${ }^{2}$, offers a very uniform and stable [75] energy response. Its radiation hardness also makes it suitable for high luminosity and high energy environments or for placement close to the interaction point. Another advantage of such a calorimeter is the ability to perform a fast detector readout - this ability is crucial for triggering. We will discuss the calorimeter-based trigger in Section 5.3.

Detailed descriptions of the LAC design, construction, and operation are found elsewhere $[62,75,76]$.

The LAC was composed of a central cylindrical barrel region (Figure 3-6) and two endcap plug-like regions (Figure 3-8). Each of the three regions was an independent mechanical and cryogenic subsystem. The LAC was placed inside the magnet coil in order to avoid placing $\approx 0.7 \lambda_{\text {int }}$ of material in front of the calorimeter. The relative dimensions of the LAC are presented in Figure 3-3 and some general engineering features are shown in Figures 3-6 and 3-8. The LAC provided full coverage in azimuth and a polar coverage of $|\cos \theta|<0.98$. The barrel LAC covered $\theta>33^{\circ}$ while the endcap covered $8^{\circ}<\theta<35^{\circ}$. This coverage translated into a nominal acceptance of $98 \%$ of the full solid angle.

The LAC barrel was supported on the detector arches by a set of four slings. The barrel was 6 meters long, had an inner radius of $1.77 \mathrm{~m}$ and an outer radius of $2.91 \mathrm{~m}$. The LAC endcaps were supported on the flux return steel doors and they wrapped around the beamline.

In order to facilitate fabrication, the LAC was segmented into a 'module' structure. The barrel section of the calorimeter was divided into a total of 288 modules. The azimuth segmentation corresponded to 48 modules while the axial segmentation corresponded to 3 modules. In the axial direction, the three modules were separated by two sets of "washers" which were part of the cryostat structure. In addition, modules were radially segmented into electromagnetic and hadronic sections. A cutaway view of a barrel electromagnetic (EM) and hadronic (HAD) module is shown in Figure 3-7. Each endcap LAC was composed of 16 wedge-shaped modules (see Figure 3-9); endcap modules were essentially functionally equivalent to their barrel counterparts, except that they incorporated both EM and HAD type modules.

The modules themselves were constructed of interleaved plates of lead and liquid argon filled gaps. The lead plates, or absorber structure, were actually alternating planes of lead sheets and segmented lead tiles. The sheets and the tiles were separated by spacers (See Figure 3-10) with the tiles arranged and properly sized into a projec-

\footnotetext{
${ }^{2}$ Unity gain means that there is no multiplication of the signal in the active medium (in our case the liquid argon). The only electrons detected are those from the primary ionization; therefore there are no additional fluctuations from the amplification process.
} 


\begin{tabular}{||l|c|c|c|c|c|c||}
\hline \hline LAC & $\begin{array}{c}\text { Cell } \\
\text { Section }\end{array}$ & $\begin{array}{c}\text { Cell } \\
\text { count }\end{array}$ & $\begin{array}{c}\text { Section } \\
X_{0}\end{array}$ & $\begin{array}{c}\text { Cell } \\
X_{0}\end{array}$ & $\begin{array}{c}\text { Section } \\
\lambda_{\text {int }}\end{array}$ & $F_{\text {samp }}$ \\
\hline \hline$e m-1$ & 8 & 0.75 & 6.0 & 0.030 & 0.24 & $18.5 \%$ \\
$e m-2$ & 20 & 0.75 & 15.0 & 0.030 & 0.60 & $18.5 \%$ \\
$h d-1$ & 13 & 0.03 & 28.4 & 0.077 & 1.00 & $7.0 \%$ \\
$h d-2$ & 13 & 0.03 & 28.4 & 0.077 & 1.00 & $7.0 \%$ \\
\hline total & 54 & - & 77.8 & - & 2.84 & - \\
\hline \hline
\end{tabular}

Table 3.1: Summary of LAC Longitudinal Segmentation.

tive tower geometry. The tiles in radially successive planes were connected together to form a tower. The EM and the HAD section were longitudinally segmented into two such towers. The lead plates were grounded and the lead tiles held at $-2 \mathrm{kVolts}$ and served as the charge collecting electrodes. Since signals were read from the tiles at high voltage, a blocking capacitor was used to read out the AC component of the signal. Details of a hadronic module cell can be seen in Figure 3-10.

The EM module lead cells were $2 \mathrm{~mm}$ thick with $2.75 \mathrm{~mm}$ liquid argon gaps. The HAD cells were $6 \mathrm{~mm}$ thick with a $2.75 \mathrm{~mm}$ argon gap. This translated into a $18.5 \%$ sampling fraction for EM modules and a $7 \%$ sampling fraction for HAD modules. The EM section was segmented into 192 towers in azimuth with a polar segmentation of 96. Towers had an equal-area projective geometry. The total number of towers in the LAC was 41,088 ; of these, 8640 were in the endcaps.

The amount of material in front of the calorimeter is sketched, in terms of $X_{0}$ and $\lambda_{\text {int }}$, in Figure 3-11. Notice the difference between endcap and barrel.

\section{The Warm Iron Calorimeter}

The WIC was both a muon identifier and a gas-iron sampling calorimeter. It was composed of both an endcap and barrel system and its massive iron structure served both as an absorber and provided a path for the magnet flux-return. The Warm Iron Calorimeter was conceived as a hadronic shower tail-catcher for the LAC. In terms of physical size it was the largest of the detector subsystems and served as a support structure for most of the detector (see Figure 3-3). The WIC barrel formed an octagonal steel outer shell for the central detector with a similar structure in the endcaps. Each octagonal structure was made up of two 'coffins', each a single steel structure with 7 alternating steel-air gap layers. The steel planes were $5.0 \mathrm{~cm}$ thick and the air gaps were $3.2 \mathrm{~cm}$ thick for a total depth of $4.2 \lambda_{\text {int }}$. The air gaps were instrumented with large arrays, 'chambers' of Iarocci tubes, which were employed in limited streamer mode with a dual tracking-calorimeter readout. Iarocci tubes were plastic ionization tubes with eight wires each running at $4.75 \mathrm{kV}$ with respect to a graphite covered sheath. The tubes were mounted on chambers where they were read- 

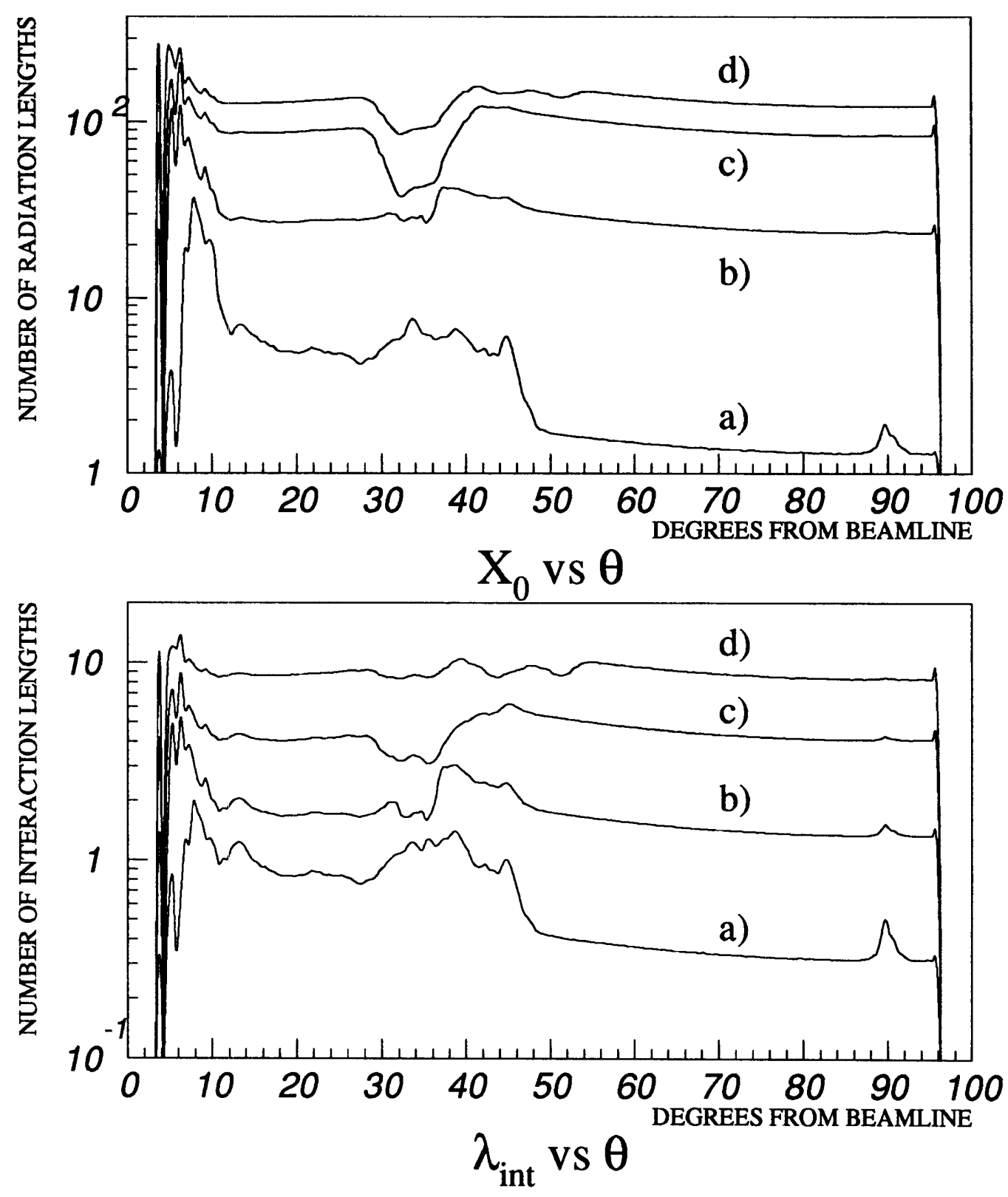

Figure 3-11: $\quad X_{0}$ and $\lambda_{\text {int }}$ as a Function of $\theta$. a) before $e m-1$, b) before $h d-1$, c) before $w i-1$, and d) total for detector. 
out, by capacitive coupling, by a set of copper strips and pads mounted on opposite sides of the chamber. Streamers in the tube (produced by single particle ionization) induced signals in the strips and the pads. The 'strips' were $0.9 \mathrm{~mm}$ copper strips which ran the length of the tube (1 strip per wire) and provided tracking information for muon identification. The pads followed the projective LAC tower geometry and collected charge which is proportional to the number of streamers in the tubes. This charge provided a coarse measurement of shower energy.

The amount of material in front of the WIC, in terms of $X_{0}$ and $\lambda_{\text {int }}$ is presented in Figure 3-11. We will study the LAC and WIC energy response in the next chapter.

\subsection{The SLD Monte Carlo}

In order to be able to extract a measurement, the 'response function' of the detector must be understood. This was accomplished by simulating both, the expected physics processes (i.e. $e^{+} e^{-} \rightarrow l^{+} l^{-}, q \bar{q}, \gamma \gamma$, etc.) and the detector response.

\section{Event Generators}

Event generators are used to generate particular physics processes. They are typically Monte Carlo programs that utilize analytic cross section calculations to obtain the desired final states. In our studies we were mainly concerned with the $q \bar{q}$ final state. We used HERWIG [50] and Lund [45] to generate hadronic events. In order to simulate the backgrounds we used KORALZ [77] $\left(\tau^{+} \tau^{-}\right)$, BHLUMI [78] $\left(e^{+} e^{-}\right)$, and

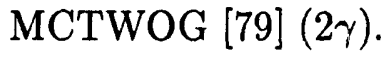

\section{Detector Simulation}

The SLD detector response was simulated using the program GEANT [80], version 3.14. GEANT carries a volume-based description of the detector and is able to describe a particle's traversal through the detector. The simulation includes effects like particle decays (when appropriate), magnetic bending, energy loss, nuclear and electromagnetic interactions, and the response of the data acquisition electronics. This scheme is essential when dealing with a complicated detector system and is completely equivalent to folding-in a detector resolution function. The detector simulation, then, 'smears' the generated event (in terms of particle species and four-momenta) into an observable event (in terms of ADC counts).

Since this analysis involves only the calorimeter system, the other detector subsystems were not included in the simulation except as passive material ${ }^{3}$. This was done in order to reduce the computer time needed for Monte Carlo event generation. In

\footnotetext{
${ }^{3}$ This means that the digitization stage was turned off for these subsystems.
} 
addition, electromagnetic and hadronic showers were simulated using GFLASH [81]. GFLASH is a fast shower parameterization which generates shower shapes as a function of energy. It parameterizes showers using overlapping Gamma functions and includes correlations between all of the shower shape parameters. Its input is the interaction point of a particle in the detector (determined by GEANT) and its output is the charge (and the charge distribution) deposited by the particle in the calorimeter.

\subsection{The SLD Event Reconstruction}

The reconstruction of the data (and Monte Carlo events) for this analysis was done, for the most part, using the standard SLD reconstruction package. The calorimeter raw data, both for real data and Monte Carlo events, consisted of a list of 'tags' (tower locations) and associated ADC counts. One can divide the reconstruction into two stages: pre-processing and clustering.

\section{Pre-processing}

During this stage hit tags were validated and their $\mathrm{ADC}$ values converted to working energies. A brief outline follows:

- Hot channels were removed: single calorimeter hits with an occupancy of greater that $2.5 \%$ during the run were removed.

- Hits in the $\theta$-bin 47 and 48 towers were removed to avoid the 'wall of fire' region (see section 5.1.2).

- Gain and capacitance corrections were applied (to correct for charge collection inefficiency introduced by the blocking capacitor).

- In order to minimize noise, a threshold energy cut was applied on a hit by hit basis. All hits below threshold (em-1 to wi-2 : 7, 7, 9, 9, 12, 12 ADC counts) were discarded.

- Energies were corrected for sampling fractions. See Table 3.1.

\section{Clustering}

Since most particles shower in the calorimeter, they usually deposit energy in more than one tower. In order to be be able to assign an energy to a particle, we must then identify and add up all of the energy corresponding to the showering particle. This was accomplished by linking neighboring hits into pre-clusters. 'Neighboring' refers to hits in contiguous towers in radial and transverse segmentation. This first step generally went too far in the clustering since real showers could overlap and thus be merged in the pre-clustering. In order to avoid this, a second pass was made in 
the clustering. At that time each cluster was analyzed to search for energy valleys regions of energy peaks with dips between them - and to split clusters that looked like merged showers. After this step, energy weighted centroids (in $\theta$ and $\phi$ ) were calculated and subsequently used as the cluster coordinates.

During the pre-clustering stage, clusters consistent with beam-induced muons (see Chapter 5) were tagged as such to be later discarded. After this initial stage we were left with a collection of clusters in an acceptance region of approximately $97.9 \%$ of $4 \pi$. We will call $E_{i}$ the energy contained in layer $i$ in a particular cluster and $E_{\text {clus }}$ we define as,

$$
E_{c l u s}=\lambda_{e m}\left(E_{e m 1}+E_{\text {em } 2}\right)+\lambda_{h d}\left(E_{h d 1}+E_{h d 2}\right)
$$

We will discuss the $\lambda_{i}$ of Eq. 3.4 later on in Section 4.5. Good clusters were then selected by two different methods,

Method 1: Required that,

- $E_{e m 1}>0$ or $E_{e m 2}>0$

- $E_{\text {clus }}>150 \mathrm{MeV}$

- Number of hits in a cluster: $N_{\text {hit }}^{\text {clus }}>1$

Method 2: Required, in addition to the requirements for Method 1 above, that the scale-independent energy correlation function,

$$
G_{c}\left(E_{e m 1}, E_{e m 2}\right)=\frac{4 E_{e m 1} E_{e m 2}}{\left(E_{e m 1}+E_{e m 2}\right)^{2}}
$$

satisfied $G_{c}>0.25$. See Figure 3-12

The main difference between methods 1 and 2 above is that method 2 took advantage of the energy correlation between the two electromagnetic layers. It was a fairly restrictive requirement - with $G_{c}>0.25$, pions that interacted at the boundary of $e m-1$ and $e m-2$ would be discarded unless $E_{\text {em } 2} \gtrsim 1.5 \mathrm{GeV}$. On the other hand the correlation requirement served as a veto on albedo-like clusters in $\mathrm{em}-1$ and on shower remnant clusters. These are precisely the type of clusters that contribute to an MC/Data disagreement when there is an improper description of detector material in the simulation. For illustration, Fig. 3-13 shows some global quantities for these two selection methods. 


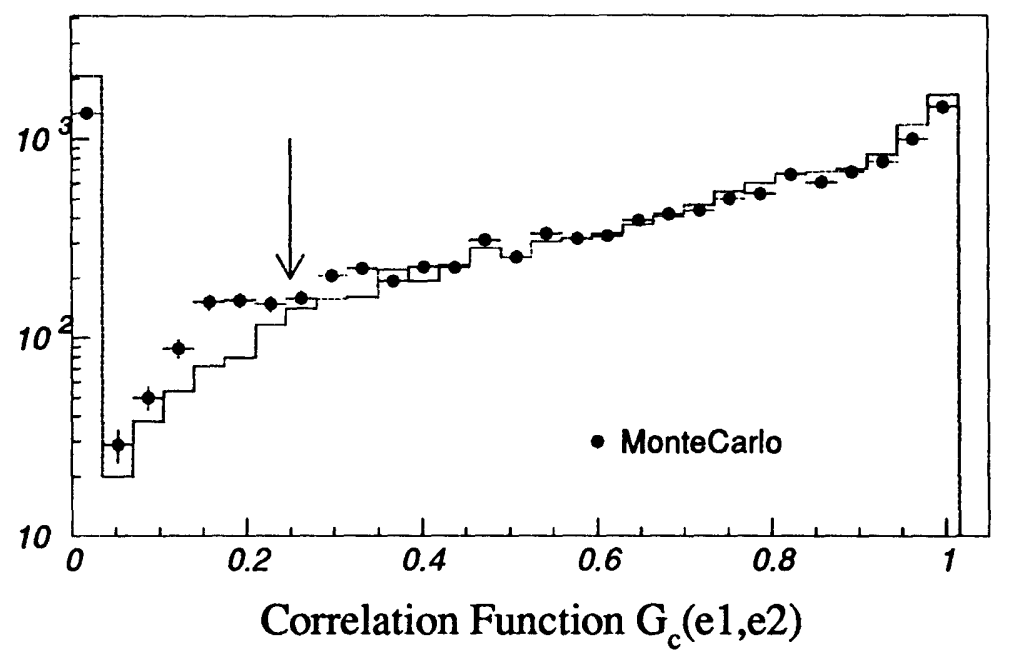

Figure 3-12: Plot of the $e m-1$ and $e m-2$ correlation function, $G_{c}$, for the Barrel region $(|\cos \theta|<0.7$ ). The cut (arrow) was designed to minimize DATA/MC disagreement.
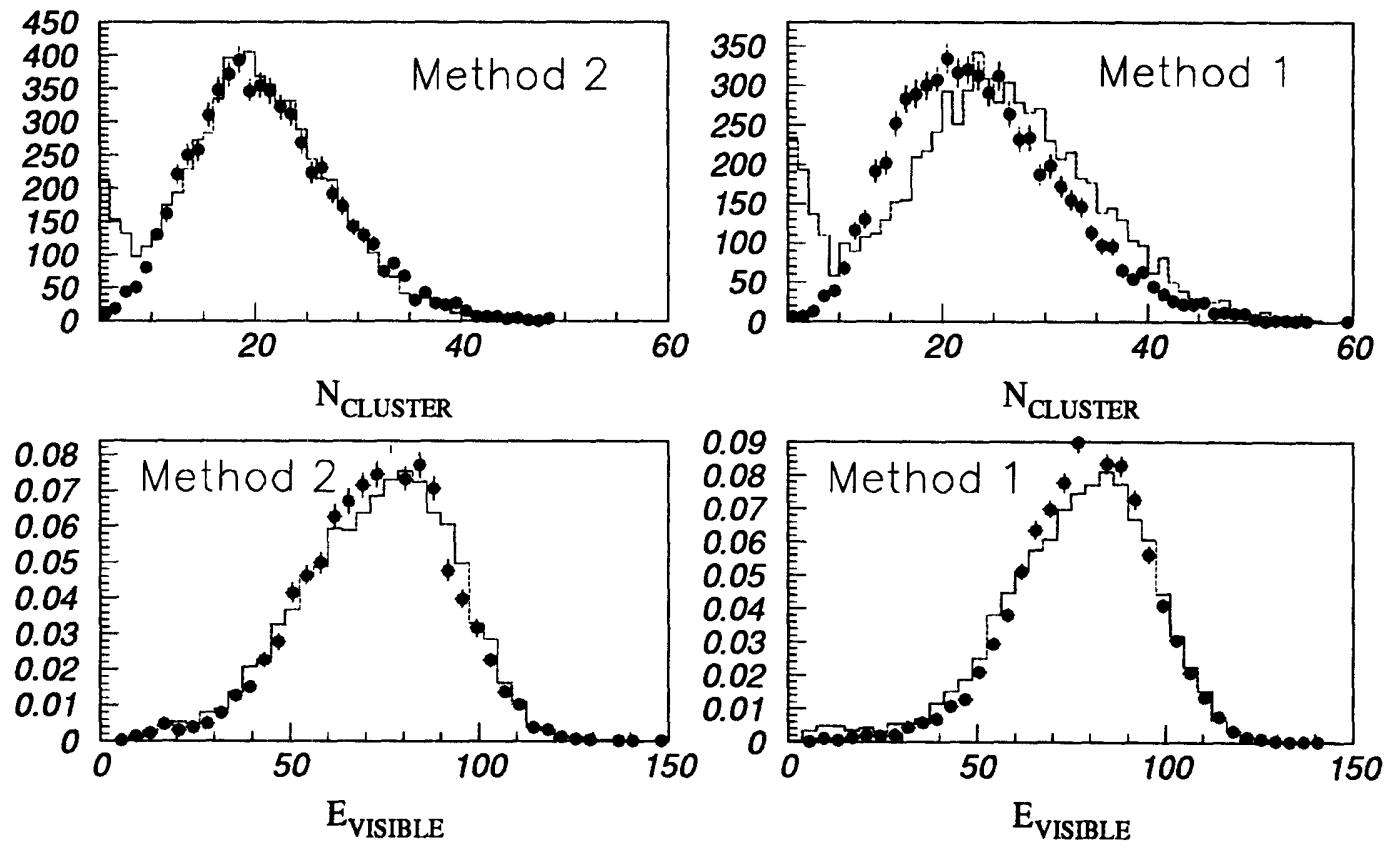

Figure 3-13: Some global observables for both cluster selection methods. The visible energy is given in $\mathrm{GeV} / \mathrm{c}^{2}$ and normalized to the total number of events. The skewness of the visible energy plots is mainly due to the known lower response in the endcap. 


\section{Chapter 4}

\section{Calibration of the SLD Calorimeter}

\subsection{Introduction}

The art of calorimetry has always been a very empirical discipline. Whereas other detector technologies have very predictable design specification to performance paths, calorimeter systems have always relied on test-beams for the experimental determination of the response function. The naturally stochastic nature of a calorimeter measurement, the complexity of the interplay between detector parameters and shower development, and the variety of interactions involved imposes the empirical approach.

The SLD test beam data was taken with a detector configuration that did not correspond to the SLD calorimeter. The SLD calorimeter system was therefore, for all practical purposes, a detector with no test beam. In order to understand the detector response to the various types of interactions, we then had to make use of the available data itself. One of the nice things about $e^{+} e^{-}$annihilation is the fact that, from a calorimetric point of view, we know the total energy of the initial state. We then used this fact and simple kinematic constraints to set the scale for the SLD calorimeter.

The data set used for the calibration consisted of roughly 10,500 hadronic and di-electron events and roughly 24,000 cosmic ray events collected during the 1992 run. The central drift chamber was used in the track-cluster studies and as a cluster veto in the $\pi^{0}$ study. The rest of this analysis used the SLD calorimeter only.

By 'SLD calorimeter' we refer to both the LAC and the WIC systems. Due to the way that signals propagate along the wires in a Iarocci tube, it turned out that in the SLD barrel SLC-induced muons would mimic true hadronic depositions in the WIC. Beam-related backgrounds in the WIC then became the dominant effect in event-toevent fluctuations. This condition, combined with the fact that the WIC was many $\lambda_{\text {int }}$ away from the interaction point, made the WIC calibration very difficult. 
A word of caution. In doing a calibration of this nature, namely 'using data to calibrate the same data', there is always the danger of introducing biases. Fortunately the nature of the measurement (jet fractions) is very insensitive to relative energy scales. We are then safe 'to first order'. Later, we will estimate the systematic effects of energy scales on event topologies by varying the calibration factors that we find in this chapter. This self-calibrating approach to doing physics is obviously not desirable; in our case, however, it is necessary.

\subsection{Scales}

We will use the word 'calibration' in this chapter to refer exclusively to a reconciliation between the observed and the expected energy for a particular process. There are many corrections in between (capacitance correction, online calibration, sampling fraction, etc) described elsewhere [82] which we will not discuss here. We are thus only interested in the intrinsic energy response of the detector.

We use the term 'scale' to refer to a conversion factor that translates a measurement of charge in the detector into a corresponding energy; in general each different type of interaction (i.e. electromagnetic, hadronic, etc.) has a different scale. If we 'know' the scale, we are then able to assign the right units of energy to an otherwise arbitrary measurement of charge. We use a related term, response or response function, to refer to the read-out energy of the detector as a function of incident momentum and angle.

We will discuss the three energy scales of interest: the single minimum ionization process (mip or $\mu$-scale), the electromagnetic process ( $\left(\frac{e}{\mu}\right)$, and the hadronic process ( $\left(\frac{\pi}{\mu}\right)$. We choose the simplest of these processes, mip, as the reference scale for the other two. The $\mu$-response can be easily calculated given the sampling fraction and various active detector parameters. As the mip factor then includes all of the charge $\rightarrow A D C$ counts conversion factors, we can then talk of the scales relative to the mip scale. We will use the words 'mip' and $\mu$-response interchangeably. Strictly speaking this is not correct since mip refers to the single-ionization response given by the minimum of the Bethe-Bloch equation, whereas $\mu$-response refers to the momentum-convoluted version with other effects like $\delta$-ray production, and bremsstrahlung (i.e., all the things that give rise to Landau tails).

A word on nomenclature: we will use the symbol ' $m i p$ ' to refer to the absolute $\mu$-scale, the term $\frac{e}{\mu}$ to refer to the relative electromagnetic scale, and the term $\frac{\pi}{\mu}$ to refer to the relative hadronic energy dependent scale. The denominator in these symbols is a reminder that we have chosen mip as our reference scale. We will also use $\frac{e}{\pi}$ for the ratio of electromagnetic to hadronic scales. We will denote by $\left.\frac{\pi}{\mu}\right)_{i n t r}$ the intrinsic energy independent hadronic scale. Finally, the term $f_{e m}$ will refer to the fraction of energy in a hadronic shower which is electromagnetic in nature. 


\section{$4.3 \mu$-Response}

Except for $e^{+} e^{-} \rightarrow \mu^{+} \mu^{-}$events, the minimum ionizing deposition in a physics event is a very small fraction, roughly $5 \%$, of the visible energy $E_{v i s}$. As already mentioned, the importance of the $\mu$-scale is that it establishes the intrinsic response of the detector to a single (on average) ionization and a natural reference scale for the electromagnetic and the hadronic responses. In addition, the very local character of mip depositions makes their use attractive for studying detector acceptance effects. One can ' $\mathrm{X}$-ray' cracks in the detector and study $d E / d X$ penetration as a function of energy.

In this section the $\mu$-scale will be determined using two different methods; we will look at cosmic rays and at minimum-ionizing single clusters in hadronic events.

\subsubsection{Cosmic Rays}

The cosmic rays used in this study were triggered by a coincidence of the WIC octants 2 and 6 (top and bottom octants in the barrel). After requiring the presence of two back-to-back tracks in the CDC in each event and that each track have at least one cluster in the calorimeter (selected with method 1) associated with it $^{1}$, there were approximately 25,000 good single tracks left. Since these cosmic rays entered at various angles of incidence and since we are interested in the intrinsic response, we must then correct for the liquid argon path length. The correction used is,

$$
E_{\text {corrected }}=\frac{E_{\text {raw }}}{\sqrt{1+\left(\cot \theta-z / R_{L A C}\right)^{2}}}
$$

where $\theta$ is the projective cluster angle, $z$ is CDC $z$-coordinate of the muon, and $R_{L A C}$ is the $\mathrm{LAC}$ radius. After requiring the cosmic pair in the CDC to be well-contained, one can study the penetration depth of muons as a function of incident momentum. One requires,

- $x^{2}+y^{2}<400 \mathrm{~cm}^{2}$

- $|z|<60 \mathrm{~cm}$

- $2 \pi>\phi_{\text {track }}>\pi$

the last requirement is necessary to ensure a lower octant track (top $\rightarrow$ bottom track). From Fig. 4-1 one can read-off the energy loss for the various layers. As can be seen from the figure, the Monte Carlo prediction agrees with the data.

The next step is then to look at individual layer depositions. To ensure that every layer is hit, from Fig. 4-1 we require $p_{\min }>2 \mathrm{GeV}$. Figure 4-2 summarizes the results

\footnotetext{
${ }^{1}$ In this case the association is done by requiring that $\left(\theta_{\text {track }}-\theta_{\text {cluster }}\right)^{2}+\left(\phi_{\text {track }}-\phi_{\text {cluster }}\right)^{2} \leq$ $(.08)^{2}$.
} 


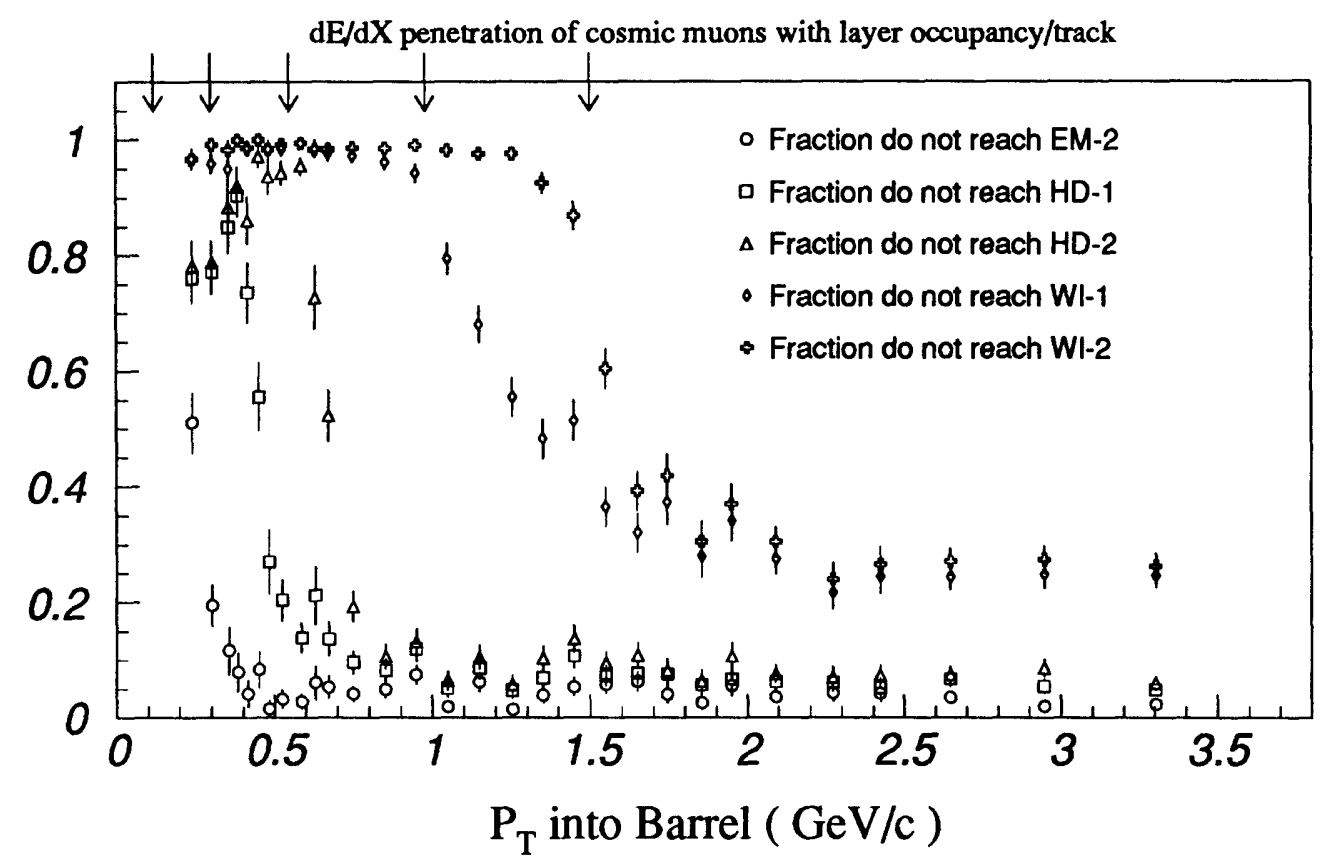

Figure 4-1: Fraction of muons with no energy deposition beyond a particular layer as a function of incident momentum $p_{t}$. Notice that the five sets of points contain two plateau regions; the transition region in between defines the $d E / d X$ loss between the two corresponding calorimeter layers. The arrows represent inter-layer boundary predicted by Monte Carlo 
of the corrected energy depositions ( $E_{\text {corrected }}$ from Eq. 4.1). As expected, the plots show fairly sharp peaks accompanied by a Landau-type tail. Each peak was fitted to a Gaussian and a Moyal function [83] independently. Results are tabulated in Table 4.1 .
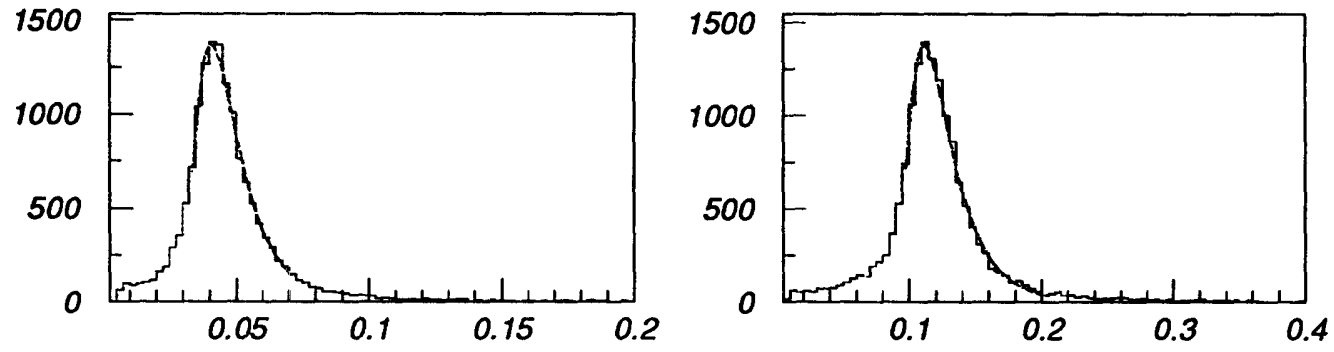

MinI in EM-1 Barrel (Cosmics, $\mathrm{GeV}$ in $\mu$-scale)

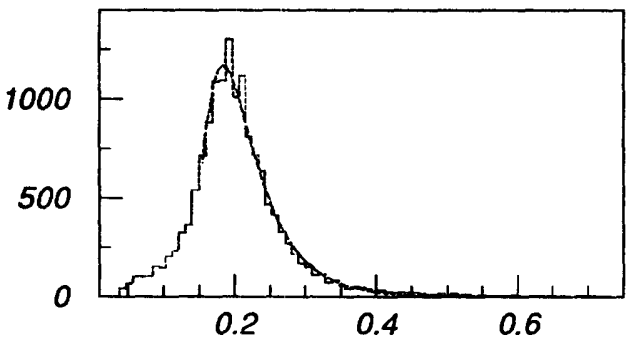

MinI in EM-2 Barrel (Cosmics, GeV in $\mu$-scale)

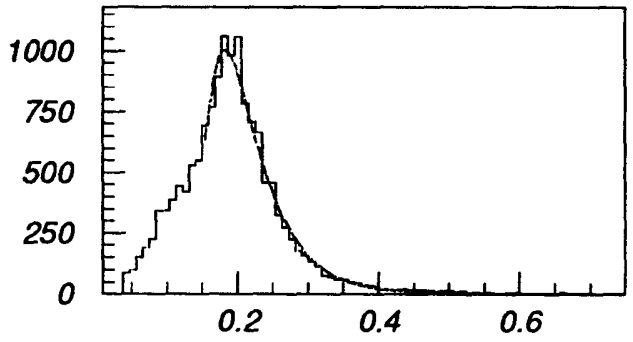

MinI in HD-1 Barrel (Cosmics, GeV in $\mu$-scale)

MinI in HD-2 Barrel (Cosmics, GeV in $\mu$-scale)
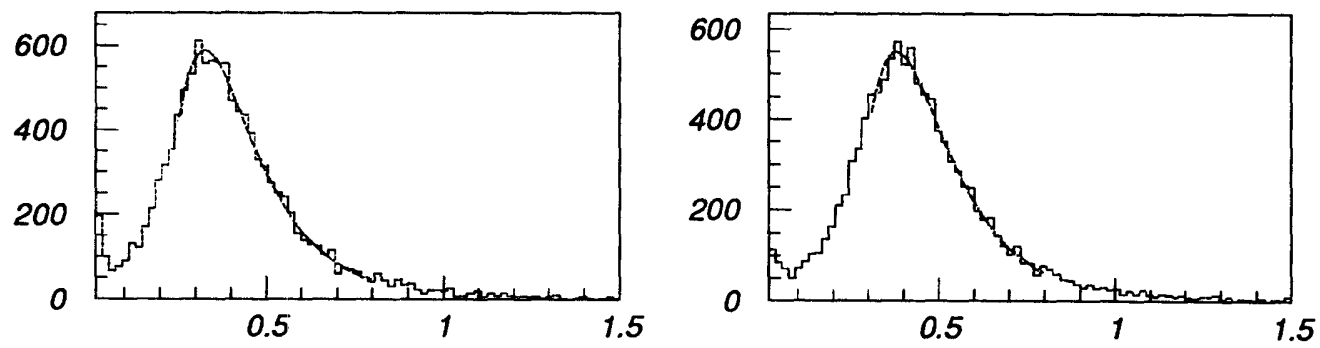

MinI in WIC-1 Barrel (Cosmics, $\mathrm{GeV}$ in $\mu$-scale)

MinI in WIC-2 Barrel (Cosmics, GeV in $\mu$-scale)

Figure 4-2: $\quad$ Layer-by-layer corrected energy deposition, $E_{\text {corrected }}$, for cosmic rays. The most-probable-value fits to a Moyal function are shown; results are summarized in Table 4.1.

\subsection{2 $\mu$-Response from single clusters}

With a total interaction length of $\lambda_{\text {int }}=0.84$ before the hadron calorimeter, roughly half of all hadrons with enough energy to penetrate to the hadronic section of the calorimeter look like muons in the electromagnetic calorimeter. Of those that survive 


\begin{tabular}{||c|c|c|c||}
\hline \hline Layer & Barrel COSMICS & ${\text { Barrel } Z^{0} \text { DATA }}^{\text {EndCap } Z^{0} \text { DATA }}$ \\
\hline \hline em-1 & $42.4 \pm 1.4$ & $44.6 \pm 1.5$ & $42.6 \pm 1.5$ \\
em-2 & $115.2 \pm 3.7$ & $114.0 \pm 3.8$ & $109.2 \pm 4.0$ \\
hd-1 & $193.1 \pm 6.2$ & $186.9 \pm 7.0$ & $187.3 \pm 7.0$ \\
hd-2 & $188.7 \pm 6.3$ & $176.4 \pm 10.4$ & $188.2 \pm 3.2$ \\
wi-1 & $347 \pm 12$ & - & - \\
wi-2 & $396 \pm 14$ & - & - \\
\hline \hline
\end{tabular}

Table 4.1: Summary of all minimum ionizing fits, in MeV. Errors shown are a combination of statistical and systematic errors. Systematic errors of $\sim 3 \%$ were estimated by performing a fit to the most probable value,mpv, with both a Gaussian and with the Moyal (see text) function. The variation due to the fit ranges and cluster selection was included in the systematic errors.

the EM calorimeter, roughly $13 \%$ leave the hadronic calorimeter intact (at normal incidence). We therefore have in the $Z^{0}$ data sample a good source of muon-like interactions in the LAC. We select mip clusters by requiring,

- at least one layer energy consistent with mip (as determined by cosmics).

- for the barrel clusters, $\left|\cos \theta_{\text {cluster }}\right|<0.65$ while for the endcap clusters, $\left|\cos \theta_{\text {cluster }}\right|>0.84$
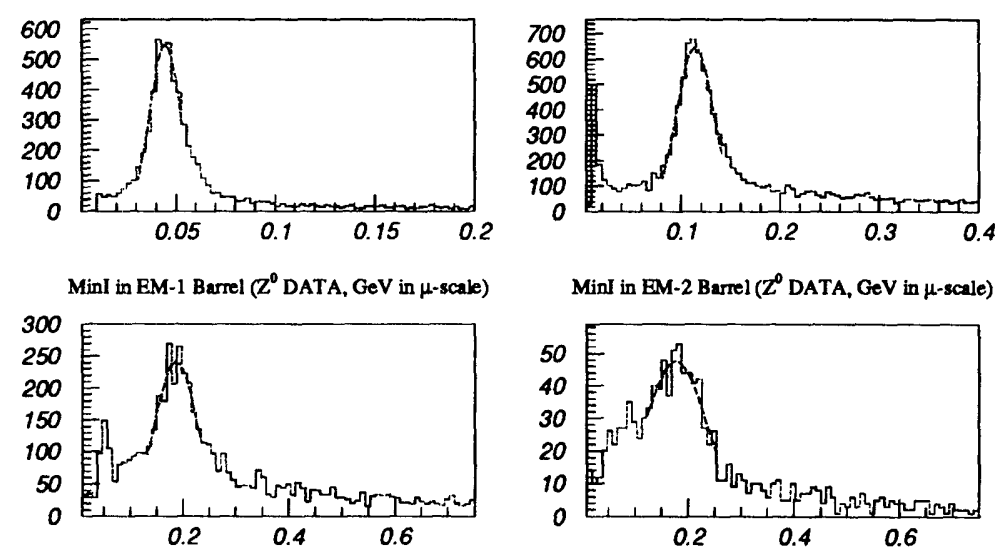

Minl in HD-1 Barrel ( $Z^{0}$ DATA, GeV in $\mu$-scake)

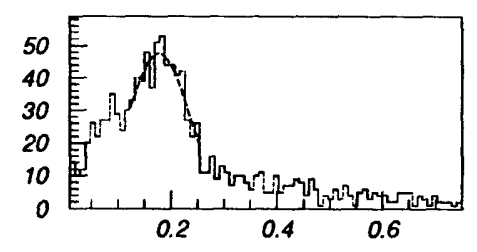

MinI in HD-2 Barrel ( $Z^{\circ}$ DATA, GeV in $\left.\mu-s c a k\right)$

Figure 4-3: Layer-by-layer corrected energy deposition, $E_{\text {corrected }}$, for barrel single mip clusters in hadronic events. The peak fit to a gaussian is shown; results are summarized in Table 4.1. 

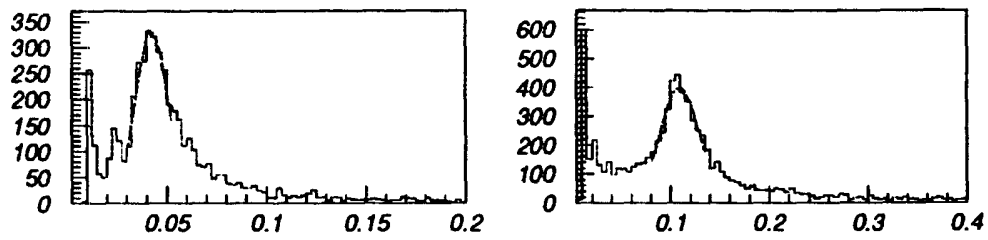

Minl in EM-1 Eodcap (Z DATA, GeV in $\mu$-scake)
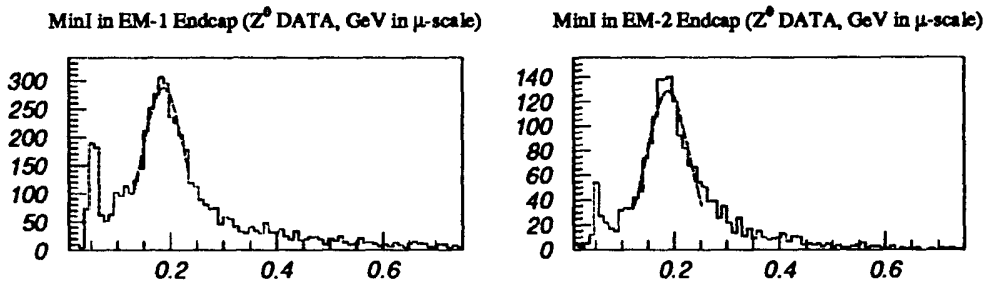

Minl in HD-1 Endcap (Z DATA, GeV in $\mu$-scale)

Minl in HD-2 Endcap ( $Z^{0}$ DATA, GeV in $\mu$-scake)

Figure 4-4: Layer-by-layer corrected energy deposition, $E_{\text {corrected, }}$, for endcap single min-i clusters in hadronic events. These endcap clusters include SLC-induced muons. The peak fit to a gaussian is shown; results are summarized in Table 4.1.

As with the energy from the cosmic ray clusters, the cluster energy is corrected to account path length differences due to incidence angle. In the case of single clusters, where they mostly originate from the IP, the correction is,

$$
E_{\text {corrected }}= \begin{cases}E_{\text {raw }} \sin \theta & \text { barrel clusters } \\ E_{\text {raw }} \cos \theta & \text { endcap clusters }\end{cases}
$$

where $\theta$ is the SLD coordinate angle from the beam-pipe. Figures 4-3 and 4-4 summarize the results for the barrel and the endcap respectively. Since hadrons traverse $\lambda_{\text {int }} \approx 3.0$ before reaching the WIC, and with just 10,000 hadronic $Z^{0}$ s, the sample of minimum ionizing hadrons which survive in the WIC is very small. In addition, the number of di-muon events and of events with hard prompt muons is also very small. For these reasons the single-cluster $\mu$-scale calibration excludes the two WIC layers.

There are a few differences in the results presented in Figs. 4-3 and 4-4 and Fig. 4-2 worth noting. The minimum ionizing clusters in a $Z^{0}$ event are overlayed on unrelated energy depositions; this inevitable overlap is seen in the tails of the distributions. As expected, the yields for single layer depositions from $Z^{0}$ clusters goes down as one looks deeper into the calorimeter. What is more interesting is that the endcap and barrel distributions are, at the $\sim 5 \%$ level, essentially the same.

One should also keep in mind that these distributions have not been corrected for the acceptance effects shown in figures 4-5 and 4-6. Fig. 4-5 shows the effect of the LAC support washers on the muon energy while Fig. 4-6 shows the effect of the inter-module gap. 


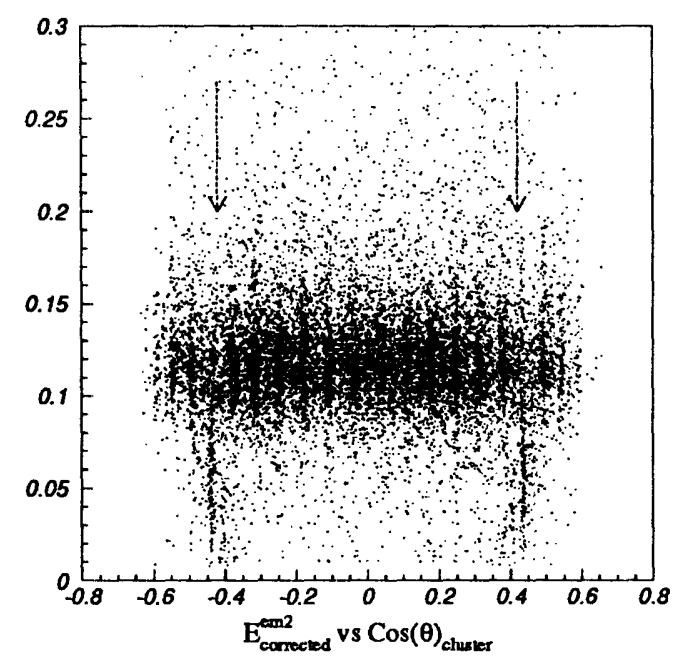

Figure 4-5: Muon energy deposition in $e m-2$ as a function of $\cos \theta$. The arrows indicate the position of the LAC washers.

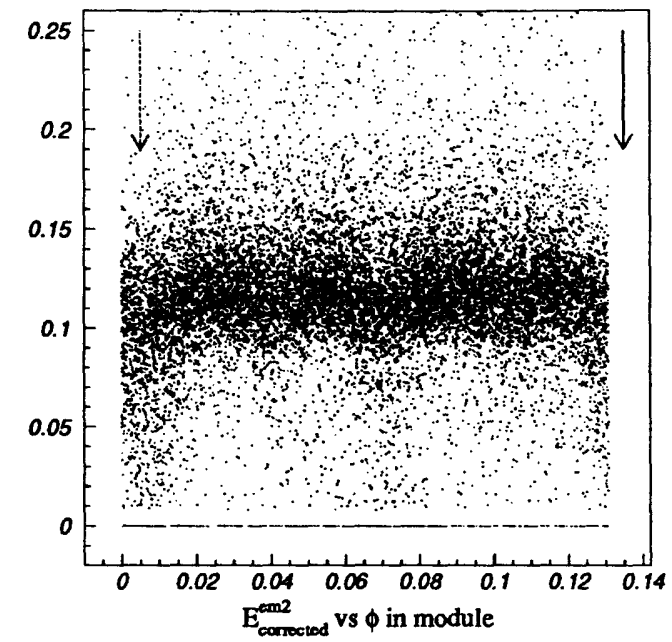

Figure 4-6: Muon energy deposition in $e m-2$ as a function of $\phi$ projected into a LAC module. The arrows indicate the boundaries of the module after a 5 milliradian CDC-LAC shift is taken into account.

\subsection{Electromagnetic Response}

The electromagnetic response was obtained by looking at $e^{+} e^{-}(\gamma)$ events and by identifying inclusive $\pi^{0} \mathrm{~s}$ in the hadronic event sample. These two methods provide measurements of the scale in an energy range of approximately $0.2 \mathrm{GeV}$ to $45 \mathrm{GeV}$. One can also use the method of identifying photon pair production of $e^{+} e^{-}(\gamma \rightarrow$ $\left.e^{+} e^{-}\right)$in the CDC and studying the track-cluster association. The yields for this last method, however, are low (when compared to the $\pi^{0}$ yields) and the $\gamma$ spectrum too soft to be useful.

\subsection{1 $e^{+} e^{-} \rightarrow e^{+} e^{-}$}

A detailed description of WAB events in SLD can be found elsewhere [84]. For our purpose all we are concerned about is to have a clean enough sample to be able to study the bulk electromagnetic response in the $\mu$-scale.

Di-electron ("WAB") events, once identified, provide a very clean and energetic test-beam for electromagnetic deposition. These events are characterized by having a high thrust, low cluster multiplicity, low energy imbalance, and a visible energy $\left\langle E_{\text {vis }}^{e+e-}\right\rangle \approx\left(\frac{e}{\pi}\right)_{i n t r}\left\langle E_{\text {vis }}^{\text {hadronic }}\right\rangle$. They are then selected by requiring,

$$
\text { - } E_{i m b} \leq 0.1 \text {, where } E_{i m b} \equiv\left|\sum_{i=1}^{n c l u s} \frac{E_{i} \vec{n}_{i}}{E_{v i s}}\right|
$$


- $f_{3}^{\max } \equiv x_{1}^{\max }+x_{2}^{\max }+x_{3}^{\max }>0.95$ where $x_{i}^{\max }$ are the normalized $\left(x_{i} \equiv E_{i} / E_{v i s}\right)$ three highest energy clusters in the event.

- less than 10 clusters with $E_{\text {clus }}>100 \mathrm{MeV}$ and more than 1 cluster with $E_{\text {clus }}>4.0 \mathrm{GeV}(\mu$-scale $)$

In this case, we have used 'method 2' to select good clusters. Figure 4-7 shows the visible energy distribution for the barrel $(\cos \theta<0.7)$ WAB events. Using $\left\langle E_{v i s}^{B h a b h a}\right\rangle=\frac{e}{\mu}\left\langle E_{v i s}^{e^{+} e^{-}}\right\rangle$, we can read off from the figure $\frac{e}{\mu}=0.69 \pm 0.02$ at $45 \mathrm{GeV}$. The error is a combination of the statistical error and a systematic uncertainty estimated by varying event selection cuts and fit ranges. The endcap region, being highly degraded due to pre-radiation, was not calibrated.

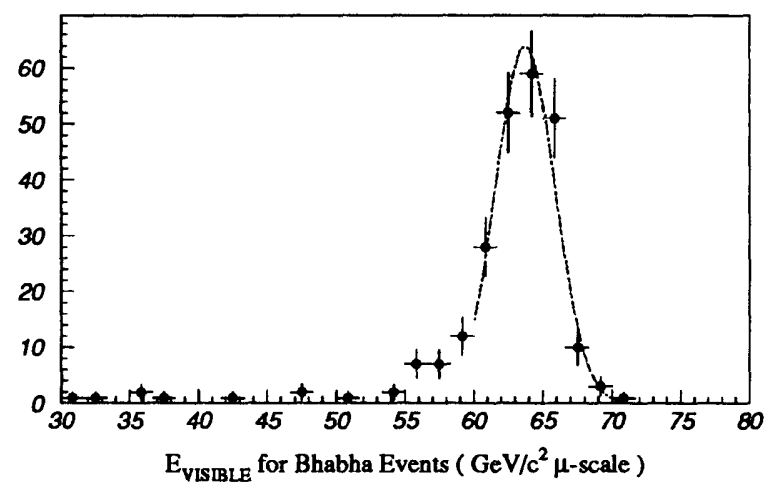

Figure 4-7: Visible energy for di-electron events in the barrel. The peak is fitted to a gaussian; units are $\mathrm{GeV}$ in the $\mu$-scale. The mean fitted value is $\left\langle E_{v i s}^{B h a b h a}\right\rangle=63.4 \pm 0.2$ $\mathrm{GeV}$ in $\mu$-scale.

\subsection{2 $e^{+} e^{-} \rightarrow e^{+} e^{-\gamma}$}

If one looks at radiative $\mathrm{WAB}$ events $\left(e^{+} e^{-} \rightarrow e^{+} e^{-} \gamma\right)$ a simple kinematic trick [85] can be used to calibrate on the photon energy. The idea is to apply the four constraints:

$$
P_{e^{-}}+P_{e^{+}}+P_{\gamma}=\left(E_{c m}, \overrightarrow{0}\right)
$$

where in Eq. 4.3 the $P_{i}$ are four-vectors. Defining the angles as in Fig. 4-9 one can write:

$$
E_{\gamma}=\frac{E_{c m} \sin \theta_{12}}{\sin \theta_{12}+\sin \theta_{13}+\sin \theta_{23}}
$$

where, as in Eq. 4.3, $E_{c m}$ refers to the center-of-mass energy $\sqrt{s}$. One immediately notices in Eq. 4.4 that the photon energy is given in terms of measured angles and the known energy. The advantage of this method is that it gives an energy measurement in terms of the well-known $E_{c m}$ provided one understands the position measurement 
given by the clustering algorithm. It also gives an energy range from 0 to $45 \mathrm{GeV}$. Radiative bhabha events are selected by requiring:

- in addition to the requirements from Sec. 4.4.1, the third highest energy cluster must be consistent with an electromagnetic deposition. (We use the same requirements as those used to find photons from $\pi^{0}$ s; see Section 4.4.3).

- the photon from above must lie in the plane of the two-highest energy clusters in the event. Thus $\overrightarrow{\mathbf{n}}_{\gamma} \cdot \overrightarrow{\mathbf{n}}_{e^{+}} \times \overrightarrow{\mathbf{n}}_{e^{+}} \leq .04$ insures the planarity of the event.

Fig. 4-8 shows the expected energy versus the observed energy for the photon in $e^{+} e^{-} \gamma$ events. The number of events in our initial sample is very small but one can see that $\frac{e}{\mu}$ is consistent with that estimated in the previous section.

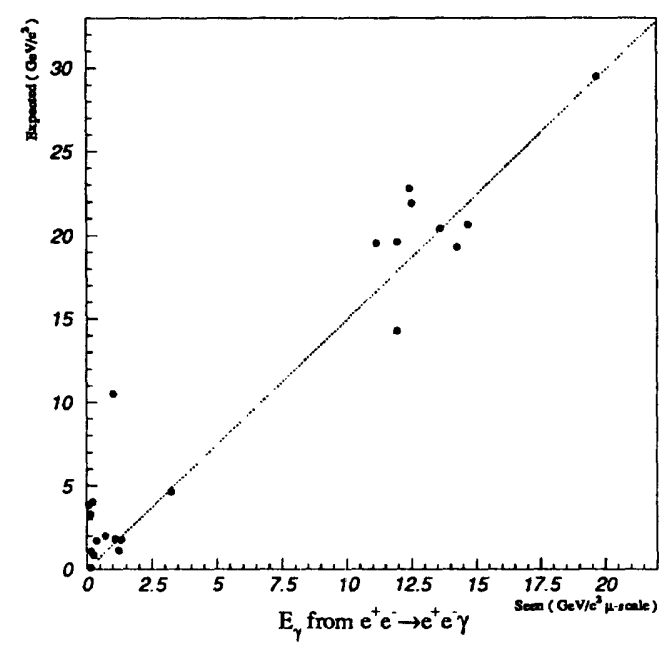

Figure 4-8: Expected vs observed energy for the photon in $e^{+} e^{-} \gamma$ events. The line (for illustration only) shows $\frac{e}{\mu}=.67$.

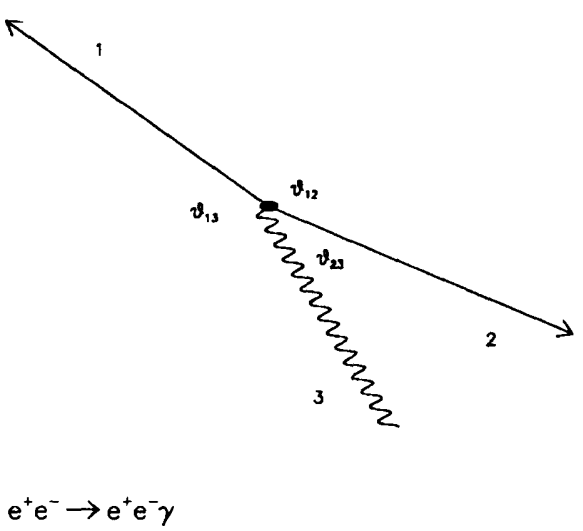

Figure 4-9: Angle Definition for Radiative Bhabhas. For radiative bhabhas, kinematics alone yields a simple relationship independent of energies.

\subsection{3 $\pi^{0} \rightarrow \gamma \gamma$}

One more way of calibrating the electromagnetic response is to identify $\pi^{0} \mathrm{~s}$ and compare their measured mass with the known mass $m_{\pi^{0}}$. The main advantages of using $\pi^{0} \mathrm{~s}$ in the calibration are that only the LAC is used in the measurement and that $\pi^{0} \mathrm{~s}$ are abundant in hadronic events. The CDC was only used to veto clusters with associated tracks.

Before proceeding to identify $\pi^{0} \mathrm{~s}$ we need to, on an event to event basis, identify neutral electromagnetic clusters. We required, 
- $\left|\cos \theta_{\text {cluster }}\right|<0.7$ to avoid the degraded endcap region.

- $\frac{E_{\text {em }}}{E_{\text {em }}+E_{\text {had }}}<0.93$ to require containment in the electromagnetic section of the LAC.

- $E_{\text {cluster }}>0.065 \mathrm{GeV}(\mu$-scale $)$

- $200 \geq N$ hit cluster $\geq 4$

- $f_{3} \geq 0.8$, where $f_{3}=$ fraction of energy carried by the 3 highest-energy hits in the cluster.

- the cluster must not be associated to a CDC track.

The last requirement, at the time of this analysis, was not very effective in reducing backgrounds. The reason was that the track cluster association efficiency was very low - typically only 3 - 4 good clusters per event had a track associated with it.

After the photon candidates were identified, events with more than 10 photon candidates were discarded in order to reduce the combinatoric background. We then proceeded to form the invariant mass hypothesis,

$$
M_{\pi^{0}}^{2}=2 E_{\gamma_{1}} E_{\gamma_{2}}\left(1-\cos \theta_{12}\right)
$$

only combining photons that were within a $60^{\circ}$ cone of each other $\left(\cos \theta_{12}>0.5\right)$. The results are presented in Fig. 4-10 for different ranges of photon energies. Each set of points was fit to a gaussian plus a third order polynomial. The combined result of the fit is $M_{\pi^{0}}^{\mu-s c a l e}=0.091 \pm 0.002$ where the uncertainty includes statistical and systematic (mainly background) errors. We thus obtain $\frac{e}{\mu}=0.67 \pm 0.015$ consistent with the previous two results.

\subsection{Hadronic Response}

The hadronic response in SLD should be, in principle, easy to obtain since most of the energy in a $Z^{0}$ event is hadronic in nature. In practice, however, the energy overlap in an event and the neutral energy component make this task very difficult.

Hadronic showers are very similar in their development to electromagnetic showers [67]; the crucial differences between them are the type of interactions governing the energy depositions and the shower multiplication. In addition, hadron showers have an electromagnetic component (due to $\pi^{0}$ s and $\eta$ 's) with generally large fluctuations. Models predict and measurements show that this electromagnetic component has a logarithmic energy dependence, $f_{e m} \approx \log E_{i n c}$. If $\frac{e}{\mu} \neq \frac{\pi}{\mu}$ (non-compensating calorimeter), then the combination of the difference in response and the large fluctuations in the electromagnetic component induce non-gaussian fluctuations in all the associated energy deposition quantities. As a result, the calorimeter resolution will 


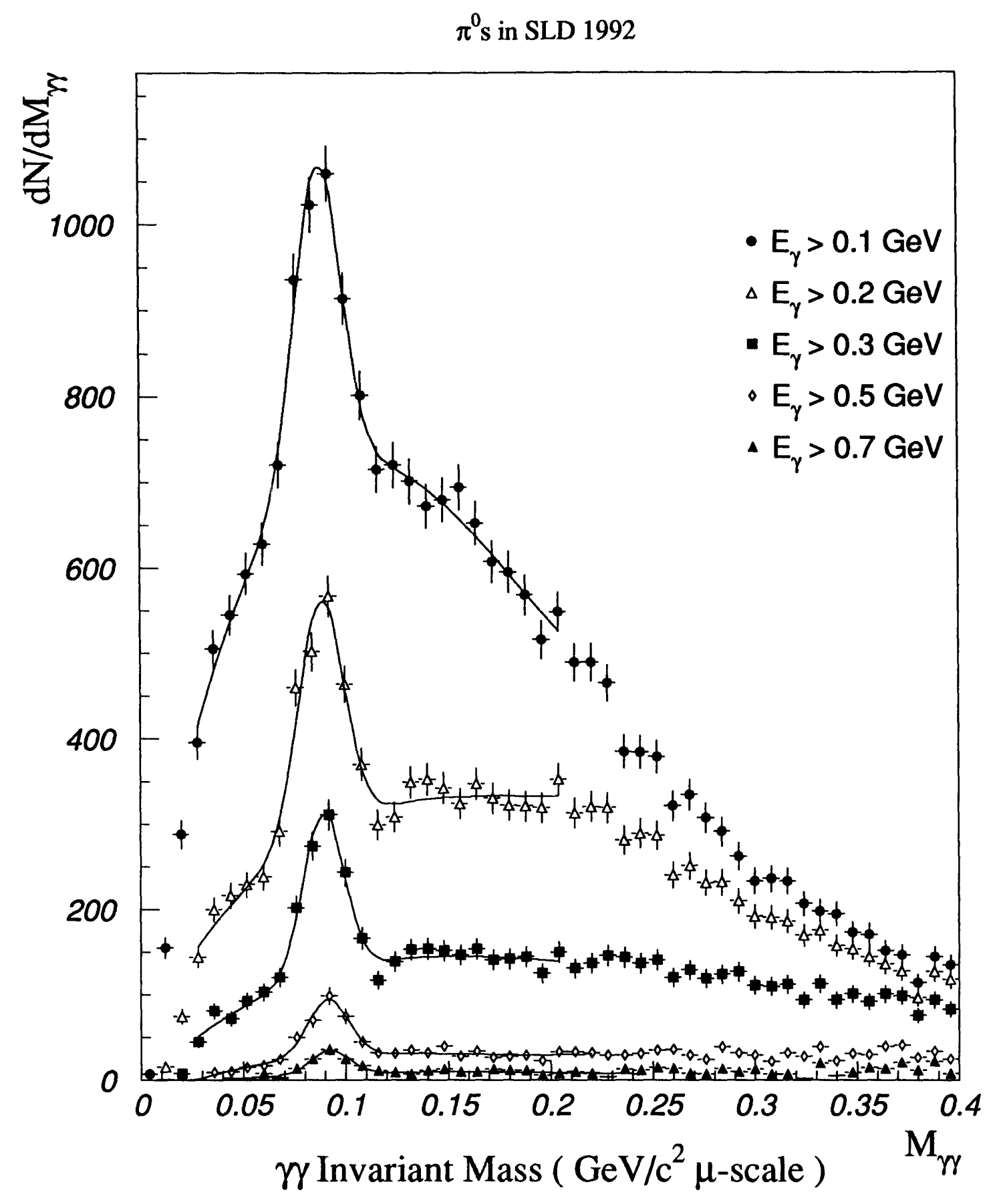

Figure 4-10: Plot of the two-photon invariant mass. The five sets of points are for the different candidate photon energies. A fit was made to a Gaussian plus a third order polynomial. Details in the text. 
deviate from the purely statistical $(\sigma / E=a / \sqrt{E}+b ; b>0)$ behavior and the response will have energy non-linearities ${ }^{2}$ that are approximately logarithmic $(\approx \log E)$ in nature.

There are a few well-defined initial states that we can use to study the hadronic response (analogous to $\pi^{0} \mathrm{~s}$ and gamma conversions). The most common ones, $K^{0} \rightarrow$ $\pi^{+} \pi^{-}$and $\Lambda \rightarrow \pi^{-} p$, can be easily identified using a vertex finding algorithm in the CDC. However, due to the 2-track resolution and to the cluster merging in the calorimeter, the usable momentum range for tracks in these two reactions is too low $(\approx 0.2$ to $1.5 \mathrm{GeV}$ ) to be useful (ionization regime dominated). We will then take two different approaches in doing this calibration: we will look at global event quantities for which any non-linearities are necessarily folded-in, and we will look at inclusive single clusters in an attempt to observe the energy-dependent behavior of these energy depositions.

Details on hadronic event selection are found in Chapter 5. For this section, it suffices to say that judicious cuts on energy imbalance, cluster multiplicity, and visible energy select a sample of about 10,000 hadronic events with an estimated total background contamination of less than $0.3 \%$. We will use clusters selected according to method 1 outlined in section 3.5.

The SLD calorimeter can be divided in three distinct segments according to sampling fraction and detector technology. We will thus group em-1 and $e m-2, h d-1$ and $h d-2$, and $w i-1$ and $w i-2$ in three distinct sub-calorimeters.

\subsubsection{Energy Flow}

By "energy flow" we refer to the relative event by event energy deposition in each calorimeter segment. Due to our limited sample of events the segments will consist of the endcap region and a crude subdivision in $\cos \theta$ of the barrel region. Each segment will then be treated on a layer by layer basis. We have already encountered the $E_{v i s}$ distribution in Fig. 3-13. As the figure shows cluster selection methods 1 and 2 yield mildly different distributions and, in addition, both methods yield distributions that are not centered around the expected $E_{\mathrm{cm}}=91.5 \mathrm{GeV}$. The first of these effects, to the extent that it affects the calibration itself, will help us in determining detector systematics in any calorimeter-based measurement. The second effect is harmless. It amounts to one overall number that can be arbitrarily chosen to center the $E_{v i s}$ distribution: $\left\langle E_{v i s}\right\rangle=f E_{c m}$.

Typically, a cluster in the SLD spans more that one layer in depth. When we form clusters we must then use a recipe for summing the individual layer energies. Following the argument of the previous section we write,

$$
E_{\text {cluster }}^{i}=\lambda_{e m} E_{e m}^{i}+\lambda_{\text {had }} E_{\text {had }}^{i}+\lambda_{w i c} E_{w i c}^{i}
$$

\footnotetext{
${ }^{2}$ This means that, for example, the energy and resolution of a $45 \mathrm{GeV}$ jet will not be the same as that of a $45 \mathrm{GeV}$ pion
} 


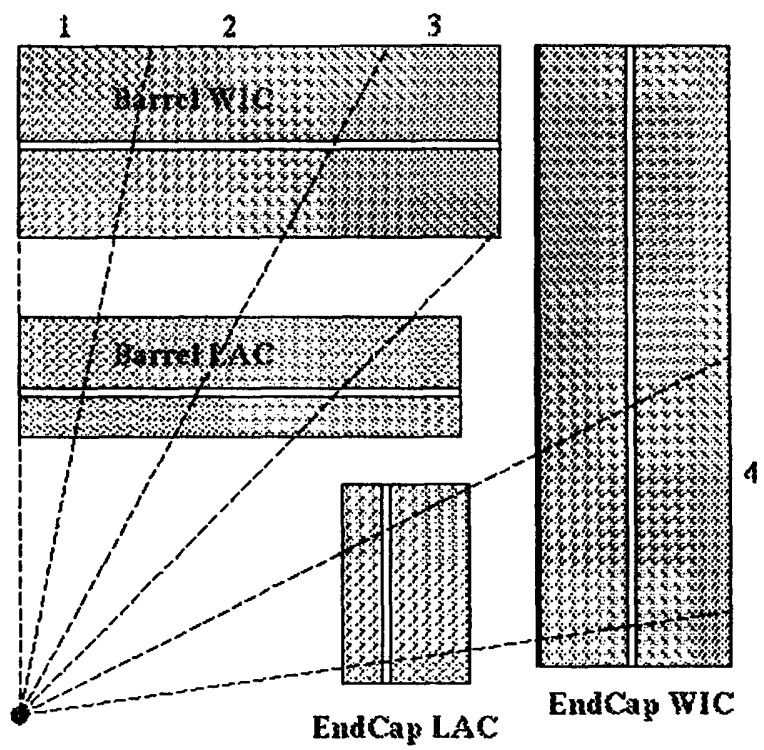

Figure 4-11: Quadrant view of the energy flow calibration regions. The regions are symmetric in $\theta$. See Table 4.2 for more details.

where the $\lambda$ 's are dimensionless factors to be determined from the data and/or Monte Carlo and $i$ is the cluster index. Using $E_{v i s}=\sum_{i=1}^{n c l u s} E_{\text {cluster }}^{i}$ and Eq. 4.6 we write,

$$
E_{v i s}=\lambda_{e m} E_{e m}^{T}+\lambda_{h a d} E_{h a d}^{T}+\lambda_{w i c} E_{w i c}^{T}
$$

If we now combine Eq. 4.7 with the requirement (to set the scale) $\left\langle E_{v i s}\right\rangle=E_{c m}$, we obtain,

$$
E_{c m}=\lambda_{e m} E_{e m}^{T}+\lambda_{h a d} E_{h a d}^{T}+\lambda_{w i c} E_{w i c}^{T}
$$

What we then have in Eq. 4.8 is a constraint equation that will help us determine the $\lambda$ factors. We proceed first by ignoring the WIC. Since the WIC is just a 'tail-catcher' for the LAC, this turns out to be a good approximation. In order to minimize the effects of tails in the energy distribution, we re-write Eq. 4.8 as,

$$
\frac{E_{c m}}{E_{e m}^{T}}=\lambda_{e m}+\lambda_{h a d} \frac{E_{h a d}^{T}}{E_{e m}^{T}}
$$

where we have removed the WIC. Armed with Eq. 4.9, and recognizing that it is just the equation of a line ${ }^{3}$ with intercept $\lambda_{e m}$ and slope $\lambda_{h a d}$, we then proceed to find the global calibration factors.

A schematic of the four detector regions that were calibrated is shown in Fig. 4-11. The quantities $E_{e m}$ and $E_{h a d}$ were found by summing all of the individual layer-bylayer cluster energies. Events were classified to belong to one of the regions (See Ta-

\footnotetext{
${ }^{3}$ If we include the WIC in Eq 4.9 then we obtain the equation of a plane and we could then have a simultaneous determination of the three $\lambda$ 's. However, the limited event sample makes this very difficult.
} 


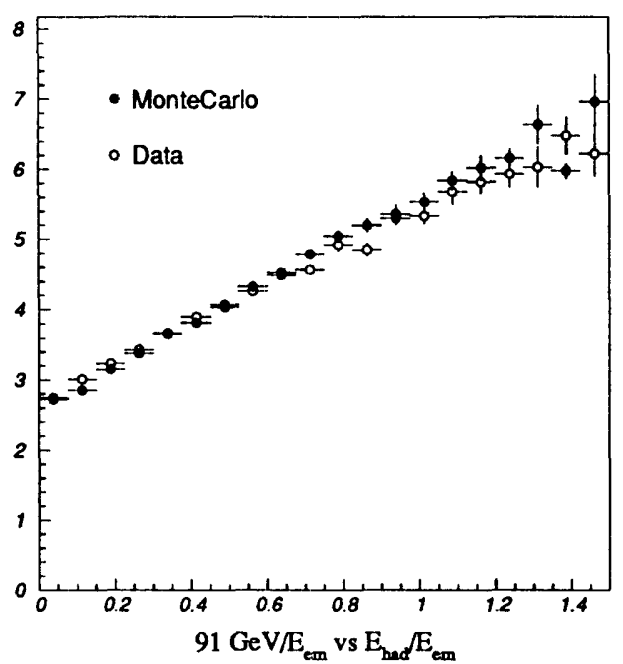

Figure 4-12: Energy flow calibration of the electromagnetic and hadronic sections of the LAC in the barrel region.

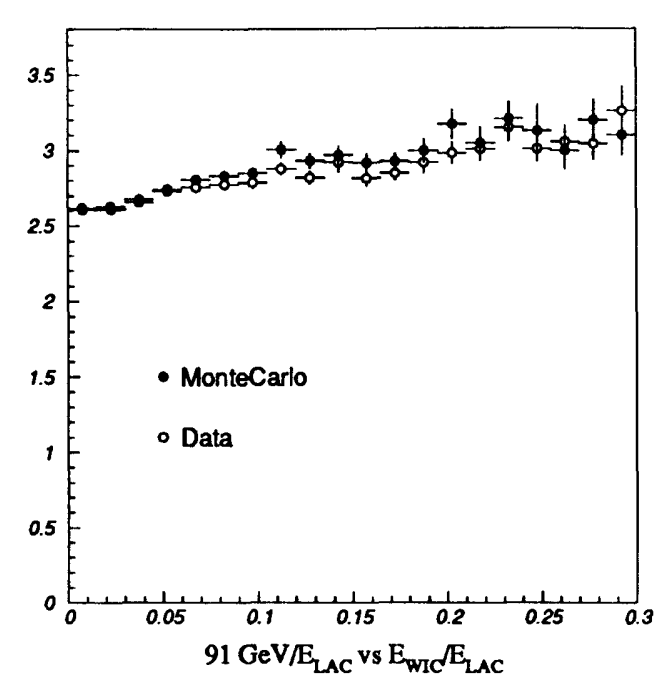

Figure 4-13: $\quad$ Energy flow calibration of the LAC and the WIC barrel region.

ble 4.2) when the thrust axis of the event was contained within the region boundaries. In order to minimize spillage of cluster energies into adjoining regions, a minimum cut in the value of thrust was placed at $T_{\min }=0.92$; this effectively collimates the event by selecting clusters which lie within a $22^{\circ}$ cone of the thrust axis. This cut, however, reduces the data sample by a factor of 3 .

Figure 4-12 shows the result of plotting the quantities defined by Eq. 4.9. A deviation from a straight line would indicate non-linearities in the energy sharing of the electromagnetic and hadronic layers of the calorimeter. No evidence is found for such non-linearities. The figure also shows the same quantities for the Monte Carlo. Any difference between the data and the Monte Carlo in the ratio $\frac{\lambda_{\text {em }}}{\lambda_{\text {had }}}$ would be an indication of a poor simulation of the longitudinal energy deposition. The data and the Monte Carlo agree within the statistics of the sample.

The same analysis was repeated for the WIC. In this case we take Eq. 4.9 and re-write it as,

$$
\frac{E_{c m}}{E_{L A C}^{T}}=\lambda_{L A C}+\lambda_{W I C} \frac{E_{W I C}^{T}}{E_{L A C}^{T}}
$$

where $E_{L A C}$ is now the total energy in the LAC. We use the $E_{L A C}$ calculated with the $\lambda$ 's from Eq. 4.9 and with both $\lambda$ 's set to 1.0 in order to estimate systematic errors in $\lambda_{W I C}$. Table 4.2 and Fig. 4-13 summarize these results.

Armed with the energy flow factors from Equations 4.9 and 4.10 one can then estimate the total energy fraction seen in each layer of the calorimeter. These results are presented in table 4.3 .

It is important to remember that these energy flow constants will in general be 


\begin{tabular}{||l|c|c||c|c||}
\hline \hline Detector Region & $\lambda_{\text {em }}$ & $\lambda_{\text {had }}$ & $\lambda_{L A C}$ & $\lambda_{W I C}$ \\
\hline \hline $0.00<\cos \theta_{t} \leq 0.25$ & $2.25 \pm 0.04$ & $3.33 \pm 0.14$ & $2.38 \pm 0.03$ & $3.40 \pm 0.60$ \\
& $2.46 \pm 0.07$ & $2.83 \pm 0.15$ & $2.33 \pm 0.02$ & $4.14 \pm 0.60$ \\
\hline $0.25<\cos \theta_{t} \leq 0.50$ & $2.44 \pm 0.04$ & $3.23 \pm 0.10$ & $2.48 \pm 0.02$ & $2.98 \pm 0.65$ \\
& $2.57 \pm 0.04$ & $2.85 \pm 0.10$ & $2.44 \pm 0.02$ & $4.00 \pm 0.90$ \\
\hline $0.50<\cos \theta_{t} \leq 0.70$ & $2.64 \pm 0.04$ & $3.12 \pm 0.13$ & $2.69 \pm 0.02$ & $2.02 \pm 0.50$ \\
& $2.74 \pm 0.04$ & $3.02 \pm 0.13$ & $2.67 \pm 0.02$ & $3.08 \pm 0.44$ \\
\hline $0.00<\cos \theta_{t} \leq 0.70$ & $2.48 \pm 0.02$ & $3.16 \pm 0.07$ & $2.54 \pm 0.02$ & $2.34 \pm 0.35$ \\
& $2.64 \pm 0.03$ & $2.81 \pm 0.10$ & $2.52 \pm 0.02$ & $3.20 \pm 0.47$ \\
\hline $0.89<\cos \theta_{t} \leq 0.97$ & $3.53 \pm 0.07$ & $3.66 \pm 0.24$ & $3.47 \pm 0.04$ & $2.84 \pm 0.76$ \\
& $3.74 \pm 0.07$ & $3.34 \pm 0.20$ & $3.49 \pm 0.03$ & $2.91 \pm 0.62$ \\
\hline \hline
\end{tabular}

Table 4.2: Results of the energy flow fits in various detector regions. Notice the slight $\theta$ dependence. For each detector segment, the upper row corresponds to the data and the lower row to the Monte Carlo. The first two columns are the calibration factors for the LAC only. Columns 3 and 4 show the factors for the LAC (with $\lambda_{e m}=\lambda_{\text {had }}=1.0$ ) and the WIC. The errors quoted are a combination of statistical errors and systematic errors estimated by varying cuts and the event sample.

\begin{tabular}{||l||c|c||}
\hline \hline Detector Region & $F_{\text {em }}$ & $F_{W I C}$ \\
\hline $0.00<\cos \theta_{t} \leq 0.25$ & $58 \pm 1 \%$ & $8.8 \pm 1.5 \%$ \\
& $62 \pm 2 \%$ & $10 \pm 1.5 \%$ \\
\hline $0.25<\cos \theta_{t} \leq 0.50$ & $64 \pm 2 \%$ & $7.1 \pm 1.5 \%$ \\
& $65 \pm 1 \%$ & $9.1 \pm 2.1 \%$ \\
\hline $0.50<\cos \theta_{t} \leq 0.70$ & $71 \pm 2 \%$ & $3.6 \pm 0.9 \%$ \\
& $71 \pm 1 \%$ & $5.1 \pm 1.0 \%$ \\
\hline $0.00<\cos \theta_{t} \leq 0.70$ & $66 \pm 1 \%$ & $5.5 \pm 0.8 \%$ \\
& $68 \pm 1 \%$ & $7.1 \pm 1.0 \%$ \\
\hline $0.89<\cos \theta_{t} \leq 0.97$ & $69 \pm 3 \%$ & $4.1 \pm 1.1 \%$ \\
& $72 \pm 2 \%$ & $4.1 \pm 0.9 \%$ \\
\hline \hline
\end{tabular}

Table 4.3: $\quad$ This table presents the mean per event energy fraction (in \%) for each calorimeter section. For each detector segment, the upper row corresponds to the data and the lower row to the Monte Carlo. Note that $F_{\text {had }}=1.0-F_{e m}-F_{W I C}$. The errors quoted are a combination of statistical errors and systematic errors estimated by varying cuts and the event sample. 
different when determined by different cluster selection methods. We were able to obtain the proper weighting factors for the calorimeter layers by exploiting the correlations inherent in the longitudinal energy-sharing of the 3 layers. These $\lambda$ 's are not fundamental properties of the detector; they can be understood as just tools to turn a raw energy into a working energy.

\subsubsection{Single Clusters}

The closest we can get to a real test-beam using $Z^{0}$ data is to study the association of tracks and clusters. SLD has such an algorithm for CDC tracks and calorimeter clusters $^{4}$ The advantage of this method is that, assuming the association is correct, we know the real incoming energy of the particle that formed the cluster. We will make no distinction between electrons and hadrons - the number of inclusive electrons (in the kinematically interesting regime) is negligible when compared to the number of charged pions.

In the course of this analysis we may be tempted to extract the global $\lambda$ 's from Sect. 4.5.1 by comparing the incident momentum $p_{i n c}^{i}$ to the deposited energy $E_{\text {dep }}^{i}=$ $\lambda_{e m} E_{e m}^{i}+\lambda_{h d} E_{h d}^{i}+\lambda_{w i} E_{w i}^{i}$. This, in fact, is wrong [86]. The reason is that the fluctuations of individual showers (having to do with the longitudinal development and the intrinsic $\frac{e}{\pi}$ ) will make the calibration factors energy-dependent. We will then use the globally optimized factors of the previous section to study the overall cluster behavior in this section. Here we will study the response and the resolution of single clusters. We will then make a direct measurement of $\frac{\pi}{\mu}$ and its momentum dependence.

Since we are dealing with quantities that have large fluctuations, we will study the ratio $E_{\mu} / p_{\text {inc }}$ where $E_{\mu}$ is the cluster energy in the $\mu$-scale of an incident particle with momentum $p_{\text {inc }}$. We then have,

$$
E_{\mu}=\left(\frac{\pi}{\mu}\right) p_{i n c}
$$

where $\frac{\pi}{\mu}$ depends on energy. If we then define,

$$
\sigma_{\mu} \equiv \delta\left(\frac{E_{\mu}}{p_{i n c}}\right)
$$

where $\delta$ refers to either the RMS width or the covariance of a distribution of Eq. 4.11, then we obtain $\sigma_{\mu}=\delta E_{\mu} / p_{\text {inc }}$ assuming $\delta p_{\text {inc }} / p_{\text {inc }} \approx 0$. Using Equations 4.11 and

\footnotetext{
${ }^{4}$ The algorithm works as follows: drift chamber tracks and associated error matrices are extrapolated outside of the CDC volume until the first layer $(e m-1)$ of the calorimeter is reached. If the track error ellipse has a sufficiently high overlap with the error circle of a cluster, then the cluster and the track are associated.
} 


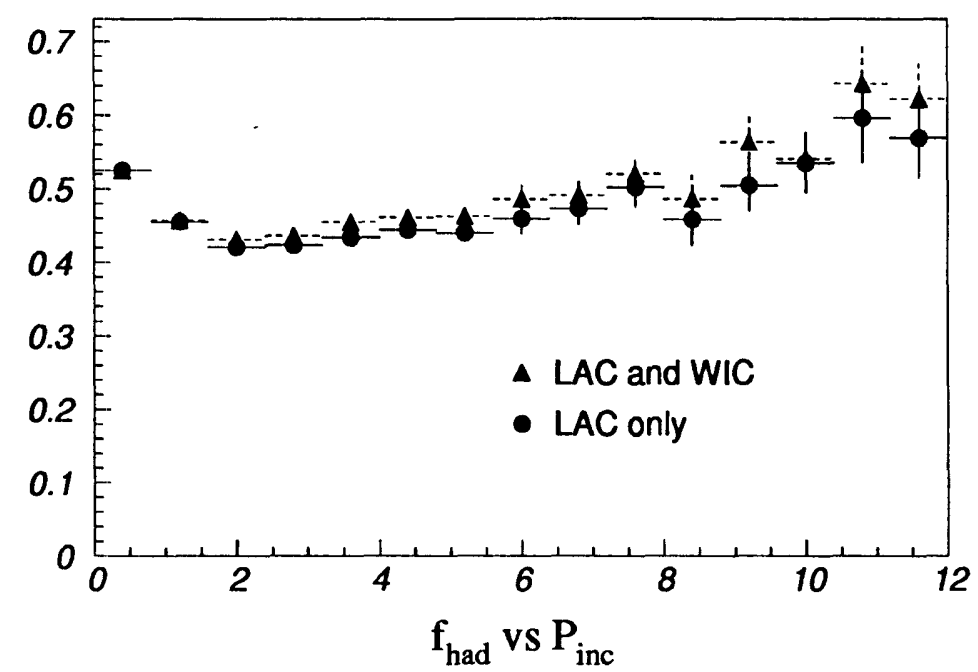

Figure 4-14: Plot of the hadronic single-particle response as a function of momentum. The energy units are $\mathrm{GeV}$ in the $\mu$-scale. The ionization-dominated regime is evident at $\approx$ $2 \mathrm{GeV}$ and below, while the onset of non-linearity due to lack of compensation is evident at higher $p$.

4.12 we then write,

$$
\frac{\sigma_{E}}{\sqrt{E}}=\frac{1}{p_{i n c}} \frac{\delta E_{\mu}}{\left(\frac{\pi}{\mu}\right)}=\frac{\sigma_{\mu}}{\left(\frac{\pi}{\mu}\right)} \sqrt{p_{i n c}}
$$

The advantage of using Eq. 4.13 is that we can get both the resolution and the response $\frac{\pi}{\mu}$ by a single fit to a distribution of $E_{\mu} / p_{\text {inc }}$. All we have to do is to divide our track-cluster sample in bins of momentum to obtain the appropriate "test-beam scan" and to fit the resultant distributions. We select clusters using method 1 and require tracks to be well contained in the $\mathrm{CDC}$ barrel (track length $>10 \mathrm{~cm}$ ). In order to minimize the background from charged particle overlap, we require that there be no charged tracks in a 130 milliradian cone around the extrapolated track. We also require no energy deposition inside a 130 milliradian cone around the cluster in order to reduce the neutral energy overlap.

Results are presented in Figures 4-14, 4-15, and 4-16. An energy response nonlinearity is evident in Fig. 4-14 indicative of $\frac{e}{\pi} \neq 1.0$. Figure 4-15 shows the distribution of $\frac{E}{p}$. The distribution is not peaked at 1.0 since, in determining the calibration factors, we required the total event energy to normalize to $E_{c m}=M_{Z^{0}}$. A marginal improvement in energy response and resolution is evident in Figures 4-14 and 4-15 when the WIC energy is included in a cluster. Figure 4-16 shows the optimum hadronic energy resolution as a function of incident momentum. The resolution is optimized since it was obtained by a simultaneous fit to both $\sigma_{\mu}$ and $\frac{\pi}{\mu}$. A realistic resolution will be given by convoluting Figure 4-15 with this plot before the fit.

At the time of this analysis there was no Monte Carlo sample available that 


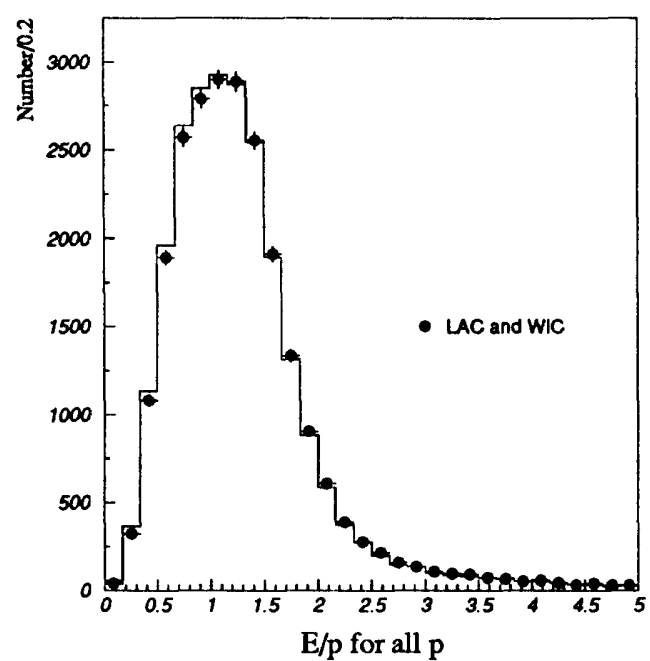

Figure 4-15: Ratio of hadronic response to incident momentum, for all momenta. The line histogram corresponds to a cluster formed with the LAC deposition only.

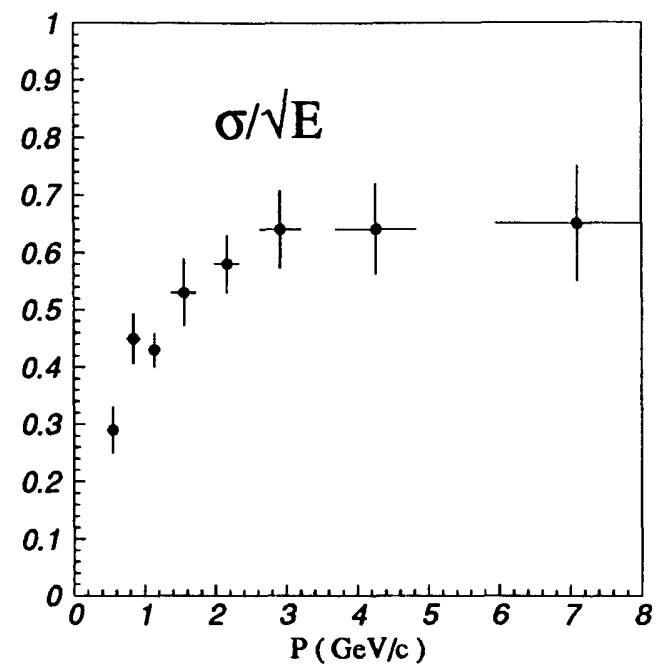

Figure 4-16: Results of the resolution fits to Eq. 4.13. The improved resolution within the ionization regime is evident below $2 \mathrm{GeV}$.

incorporated a correct implementation of $\frac{e}{\pi} \neq 1.0$ with a simulation of the tracking; for this reason, no Monte Carlo results are presented for these figures. Instead, we can look at the inclusive spectrum of clusters. The cluster energy spectrum is very sensitive to quantities like $\frac{e}{\pi}$ and $\frac{\pi}{\mu}$; one can in principle use the Monte Carlo to determine these quantities. A comparison between the data and the Monte Carlo is presented in Figure 4-17.

All we are left to do now is to determine the factor $\frac{\pi}{\mu}$. It is clear from Figure 4-14, however, that this is not an easy task since $\frac{\pi}{\mu}$ is energy-dependent. In order to extract a meaningful value of $\frac{\pi}{\mu}$ (more precisely, $\left.\frac{\pi}{\mu}\right)_{i n t r}$ ) we must then deconvolute the contribution of the electromagnetic component from Figure 4-14 with a parameterization as in Ref. [72]. We do not have the number of events to do such a measurement but we can certainly place limits on $\frac{\pi}{\mu}$ in two other ways. If we redo Figure 4-15 in the $\mu$-scale we obtain for an overall $\frac{\pi}{\mu}, \frac{\pi}{\mu}=0.43 \pm 0.02$.The quoted error includes systematic errors of a gaussian fit to the peak, estimated by varying cuts and fit ranges. This is, of course, an overestimate of $\left.\frac{e}{\pi}\right)_{\text {intr }}$ since both the high momentum and the ionization regimes will increase the effective response.

One may take a different approach and, assuming a good Monte Carlo simulation, ask what $\left.\frac{e}{\pi}\right)_{\text {intr }}$ is necessary to reproduce the data. We will address this issue in the next section. 


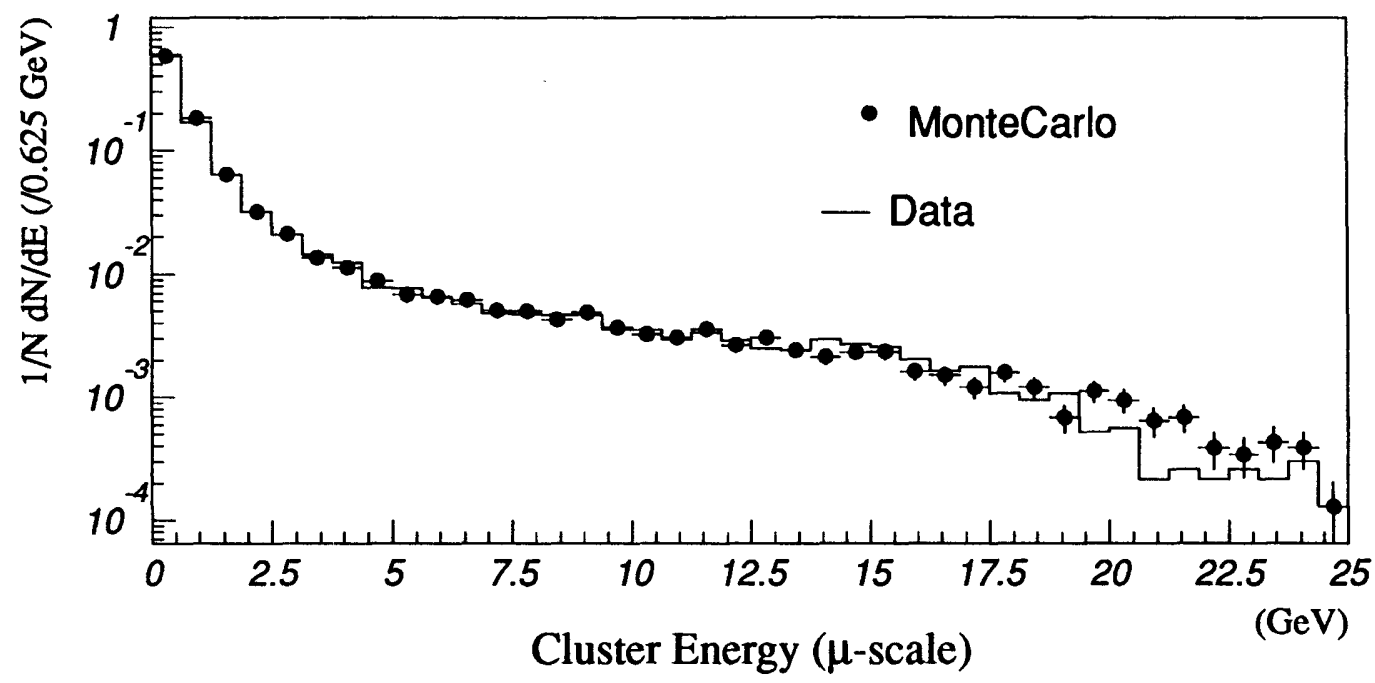

Figure 4-17: Energy distribution of inclusive clusters for both barrel and endcap. The units are $\mathrm{GeV}$ in the $\mu$-scale. This plot contains all selected clusters in 900 events for both data and Monte Carlo. The shape of this distribution is sensitive to $\frac{e}{\pi}$.

\subsection{Tuning of the SLD Monte Carlo}

The most important element in successfully tuning a Monte Carlo to reproduce data is to be able to find a set of tuning variables that are 'blind' to the underlying physics. This requirement is essential since tuning only involves ${ }^{5}$ trying to reproduce the instrumental effects - things like energy response of the detector, acceptance effects, and detector resolution effects. In an ideal situation, as we have stated, we would rely exclusively on a test beam to give us a set of well understood response functions for our calorimeter. We must instead rely on the knowledge of the initial state energy and on the 'visible particles' generated by the Monte Carlo generators.

The SLD fast shower parameterization Monte Carlo was tuned to reproduce the data. The tuning was performed in three stages. In the first stage, the electromagnetic response $\frac{e}{\mu}$ and the mip response in the Monte Carlo were fixed to reproduce the data. The trickier $\frac{\pi}{\mu}$ response was left for the second stage. In the second stage of the tuning, sets of $\approx 1200$ Monte Carlo events ${ }^{6}$ were generated with 'intelligently' chosen parameter sets. A 'parameter set' consisted of a set of Monte Carlo control variables of the shower parameterization and of the detector response. The parameter

\footnotetext{
${ }^{5}$ There is another type of tuning which involves optimizing the physics parameters of a phenomenological model (Monte Carlo). It is essential, in order for this 'second order' tuning to be valid, that instrumental effects are well reproduced by the Monte Carlo

${ }^{6}$ The Monte Carlo sets were overlayed with luminosity weighted minimum bias events to simulate background conditions. The 'minimum-bias' event sample used spanned the whole 1992 run.
} 

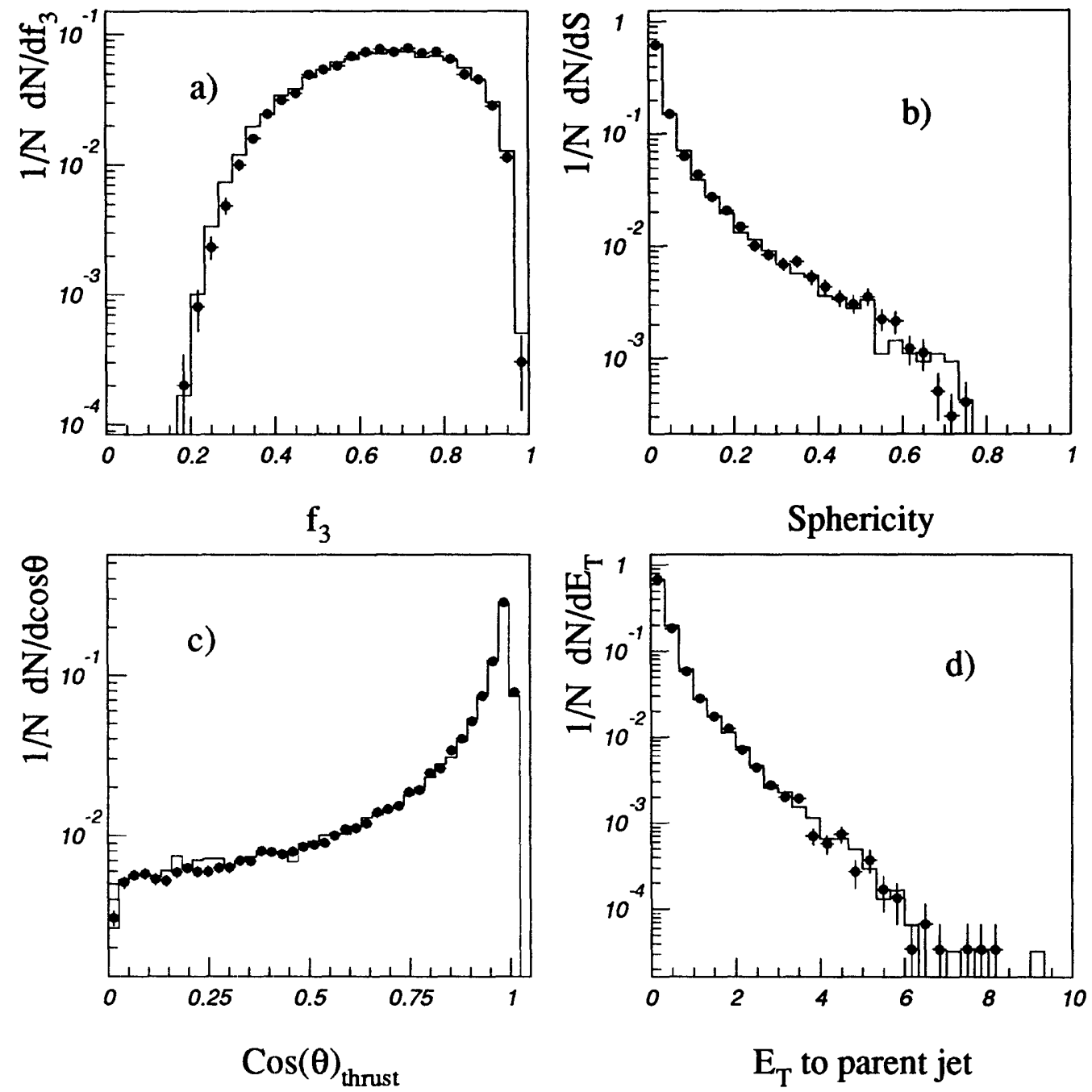

Figure 4-18: Comparison of a few observables in Monte Carlo and data. The data is represented by the solid circles and the the Monte Carlo by the line histogram. a) shows $f_{3}^{m a x}$ as defined in Section 4.4.1, b) the event sphericity (see Appendix A), c) $\cos \theta$ distribution of all clusters with respect to the thrust axis, and d) transverse energy component of clusters with respect to their parent jet for events classified as 3-jet events by the JADE algorithm (see Chapter 6). Energy units are in the mip scale. 
set included:

- transverse shower size

- inter-channel cross talk

- intrinsic $\frac{\pi}{\mu}$ response and the $\frac{e}{\pi}$ ratio

- number of energy spots per shower or shower 'foaminess'

- acceptance 'fudge' in the EndCap region

In addition the required minimum hit energies (thresholds) in the reconstruction were optimized to a value that minimized Monte Carlo-data disagreement without discarding too much information. After the events were reconstructed, histograms were made of fairly uncorrelated quantities like hit spectra, cluster spectra, cluster multiplicity, trigger quantities, visible energy, etc. The histograms corresponding to a particular parameter set were then compared to those corresponding to the 1992 data set. The Kolmogorov test was then used (most of the time) to compare sets of histograms. The Kolmogorov [87] test is a robust histogram comparison test that returns the probability that two experimental distributions (i.e. histograms) are drawn from the same parent distribution. It is sensitive to both the normalization and the shape of a histogram. Based on the results of this test and on 'visual inspection' of the distributions, an optimum parameter set was chosen.

The third stage of the tuning involved generating larger samples of Monte Carlo events to optimize the most sensitive parameters. The ultimate limitation at this stage was the CPU time available. At the point at which the distributions 'were not getting any better' and the statistical limitation of the data sample was evident, the procedure was stopped. Figure 4-18 shows a comparison between the tuned Monte Carlo and the data sample for a few observables. The upper two plots are global observables ( 1 entry per event), whereas the lower two are single cluster quantities. Fairly good agreement is evident for both observable types.

It should be remembered that the tuning was performed on the 10,000 events available at the time. Therefore, even assuming a 'perfect' tuning, the detector simulation will have systematic effects that are comparable to the statistical fluctuations inherent in such a sample. We have tried to avoid biases in our tuning procedures by using various uncorrelated and physics-blind variables. The possibility remains, however, that the chosen variables are well reproduced in the Monte Carlo but that some of the internal correlations are not.

\subsection{A Brief Comment on $\frac{e}{\pi}$}

The same arguments about $\left.\frac{\pi}{\mu}\right)_{\text {intr }}$ that we used in the previous section apply in this section to $\frac{e}{\pi}$. In fact, once $\frac{e}{\mu}$ is known, a determination of one of these factors implies the determination of the other one. 


\begin{tabular}{||l|c||}
\hline \hline Quantity & Result \\
\hline \hline$\frac{e}{\mu}$ & $0.68 \pm 0.015($ stat $) \pm 0.01($ syst $)$ \\
$\left.\frac{\pi}{\mu}\right)_{\text {intr }}$ & $0.415 \pm 0.001($ stat $) \pm 0.02($ syst $)$ \\
$\frac{e}{\pi}$ & $1.65 \pm 0.04($ stat $) \pm 0.08($ syst $)$ \\
\hline \hline
\end{tabular}

Table 4.4: Summary of energy scale measurements. Statistical and systematic errors are included where noted.

By tuning on quantities sensitive to $\left.\frac{\pi}{\mu}\right)_{\text {intr }}$ and $\frac{e}{\pi}$ it was determined that $\left.\frac{\pi}{\mu}\right)_{\text {intr }} \approx$ 0.41 and $\left.\frac{e}{\pi}=\frac{e}{\mu} / \frac{\pi}{\mu}\right)_{\text {intr }} \approx 1.7$. Once a Monte Carlo sample with the correct $\frac{e}{\pi}$ simulation and tracking is available, it will be possible to determine these factors with more precision and to realistically assign errors to them.

For now, we can say that $\left.\frac{\pi}{\mu}\right)_{\text {intr }}=0.415 \pm 0.001 \pm 0.015$ (where the uncertainties are statistical and systematic, respectively) and $\frac{e}{\pi}=1.65 \pm 0.04 \pm 0.08$ (where the uncertainties are statistical and systematic, respectively).

\subsection{Summary}

We have seen that by looking at processes with well understood initial states we were able to set the scale in the SLD calorimeter. We then proceeded to study the energy response as a function of incident momentum and into different detector regions. This study yielded an estimate for the ratio $\left.\frac{e}{\pi}\right)_{i n t r}$. We also determined that the tuned Monte Carlo reproduces the detector response fairly well, considering that we only had 10,000 events to utilize. We also established the $\mu$-scale in Table 4.1 and determined $\frac{e}{\mu}$ and $\frac{\pi}{\mu}$. These results are summarized in Table 4.4. 


\section{Chapter 5}

\section{Triggering and Event Selection}

In this chapter we discuss the procedure by which we validate our data sample. We define the term 'event' as any observation recorded with the detector. Most of these observations were irrelevant for this analysis since we only used hadronic events. We refer to these uninteresting events as our background. We also will see that the background can be classified according to its source; it can either be physics-related (non-hadronic final state of the $Z^{0}$ ) or beam-related. At times we will use the term 'minimum bias event'. A minimum bias event can be thought of as a snapshot of the detector in full operation taken at a random time during the run.

In the process of identifying good events (our hadronic sample) we can distinguish three stages: triggering, filtering, and selection. The trigger stage (described in Ch. 3) is hardware-based and its aim is to maximize the event throughput while minimizing biases in the data sample. We will see that in practice these requirements imply a low signal to noise ratio $(S / N)$. The second stage, filtering, is a software attempt to improve the $S / N$ ratio so that we can have a more manageable data sample. We will go from $\approx 1,000,000$ triggers to $\approx 20,000$ filtered triggers during this stage. The filtering will leave us with a sample small enough that we can fully reconstruct to perform a final selection or validation of our hadronic event sample.

By using only scalar quantities (energy sum, number of hits) the trigger and filter stage keep final event sample biases to a minimum. We will then purposely bias our sample by using more topologically-oriented quantities (e.g. energy imbalance, number of clusters) in order to select hadronic events. Before going into more detail on the above procedure, we will briefly review the properties of the 1992 run. 


\subsection{Properties of the 1992 Run}

\subsubsection{Luminosity in the SLD}

The luminosity in a $e^{+} e^{-}$collider is generally measured using Bhabha scattering in the forward region; this process is shown in Figure 5-3 as one of our hadronic backgrounds. The SLD device that measured the Bhabha process, the luminosity monitor-small angle tagger (LMSAT), was described in Chapter 3. Here we'll just briefly describe how the luminosity was obtained [65] even though for this analysis an absolute luminosity determination is not essential. What is more important is that we understand (i.e. be able to reproduce with a Monte Carlo simulation) any biases in the data sample.

The tight and highly dense energy signatures of the Bhabha-induced electromagnetic showers were easily recognized in the LMSAT. Since the detector was so close to the beam-pipe where it was susceptible to beam-backgrounds and missalignments, care had to be taken in counting the effective number of Bhabha events. The LMSAT was then divided into two fiducial regions: a 'gross' region and a 'precise' region. Using $N_{B h a b h a}^{\text {gross }}$ and $N_{B h a b h a}^{\text {precise }}$, an effective number of events, $N_{B h a b h a}^{\text {effective }}$, was constructed. The Monte Carlo was then used to calculate a corresponding effective cross section, $\sigma_{\text {eff }}$, taking care to simulate the background processes. Finally, an integrated luminosity was calculated:

$$
\int \mathcal{L} d t=\frac{N_{B h a b h a}^{\text {effective }}}{\sigma_{\text {eff }}} .
$$

The measurement of the luminosity for the 1992 polarized run yielded $\int \mathcal{L} d t=$ $385.37 \pm 2.47 \pm 3.89 \mathrm{nb}^{-1}$ where the first error is statistical and the second error is systematic. Later we use this number to estimate the triggering and selection efficiency.

Luminosity events were well contained in the LMSAT. The LAC was therefore devoid of any physics content during these very forward events. This fact allowed us to treat the luminosity events as monitors of the run conditions in the LAC and the WIC. We treated such events as luminosity-weighted minimum bias events. Since these events were properly weighted, we were justified in overlaying the luminosity events on the Monte Carlo events in order to obtain a realistic simulation of the run conditions and the physics events. The details of the Monte Carlo generation and the reconstruction are given in Section 3.4.

\subsubsection{Beam-Related Backgrounds}

The SLC (described in Ch. 3), being a single-pass machine, did not enjoy the intrinsic stability of a storage ring facility. In the SLC one could have a catastrophic loss of one bunch (or a fraction thereof-with $\approx 10^{10}$ particles per bunch one can have a fairly good beam with a background-producing tail), while either the next or the previous 
bunch was fine. As the run progressed, various trigger vetoes were implemented to minimize this problem and reduce the data acquisition dead-time.

A more common mode of background production was stray electrons or positrons from the phase-space tail of a bunch striking a collimator upstream from the interaction point. In this case a jet of muons was produced, mainly by the Bethe-Heitler mechanism, and travelled down the arc in the direction of the SLD. Most of these muons did not reach the detector; they were either absorbed in the ground or deflected off the tunnel by the muion-spoiler magnets (See Ch. 3). Some of these muons, however, were trapped in the beam optics, travelled down the SLC arcs, and struck the SLD detector downstream. A successful model for this process was developed for the Mark II [88] detector at SLC and later implemented for the SLD [89]. Simulations of this model predicted mean muon energies (at the entrance of the WIC) of $\approx$ $5 \mathrm{GeV}$. This aspect is unique to the SLC and years of running have demonstrated the difficulties in ridding the collider of such backgrounds.

Another type of SLC-related background had its source inside the detector. The strong field of the final focus triplet magnets induced synchrotron emission in the beam. One would then see a halo of electromagnetic-like background in the detector elements closest to the beampipe. This "wall of fire" is evident in Fig. 5-1 as a ringlike series of hits in the innermost (in $\theta$ ) layer of the LAC. The figure also shows the presence of upstream muons in the calorimeter.

Fortunately, the two types of beam backgrounds that we have described had distinct signatures. When muons entered the detector they first hit the WIC endcaps. The typical muon that would hit the WIC had enough energy to traverse SLD from endcap to endcap. The geometry of the active layers in the endcap (for both the LAC and the WIC) ensured that the background signatures in the endcap region were nominally minimum ionizing-like. In the barrel region, however, the active layers for the WIC and the LAC were shell-like volumes parallel to the beam axis and thus parallel to the background trajectories. A muon could then be trapped in an active layer gap of the LAC (liquid argon), for example, and leave an ionization trail from one edge of the barrel to the other. Since the magnetic field is solenoidal, bending effects for these muons were minimal. Typically though, the muons would enter at a slight angle to the beamline and then deposit mip-like energies in a few adjacent towers. Such trails can be seen in Figure 5-1. Not shown in the figure are the signatures in the WIC calorimeter.

The WIC calorimeter had the highest effective area of all the detector subsystems. The WIC endcaps were the first detector elements to be hit by the SLC muons. By virtue of their area, they effectively served as a shielding to the other subsystems for both low energy muons and the soft electromagnetic component. The WIC barrel in general received a somewhat higher background dosage than the LAC barrel. The higher WIC noise was due to the fact that many times the SLC operators were able to reduce the LAC backgrounds by tuning the SLC orbits such that the muons would be deflected into the WIC. 

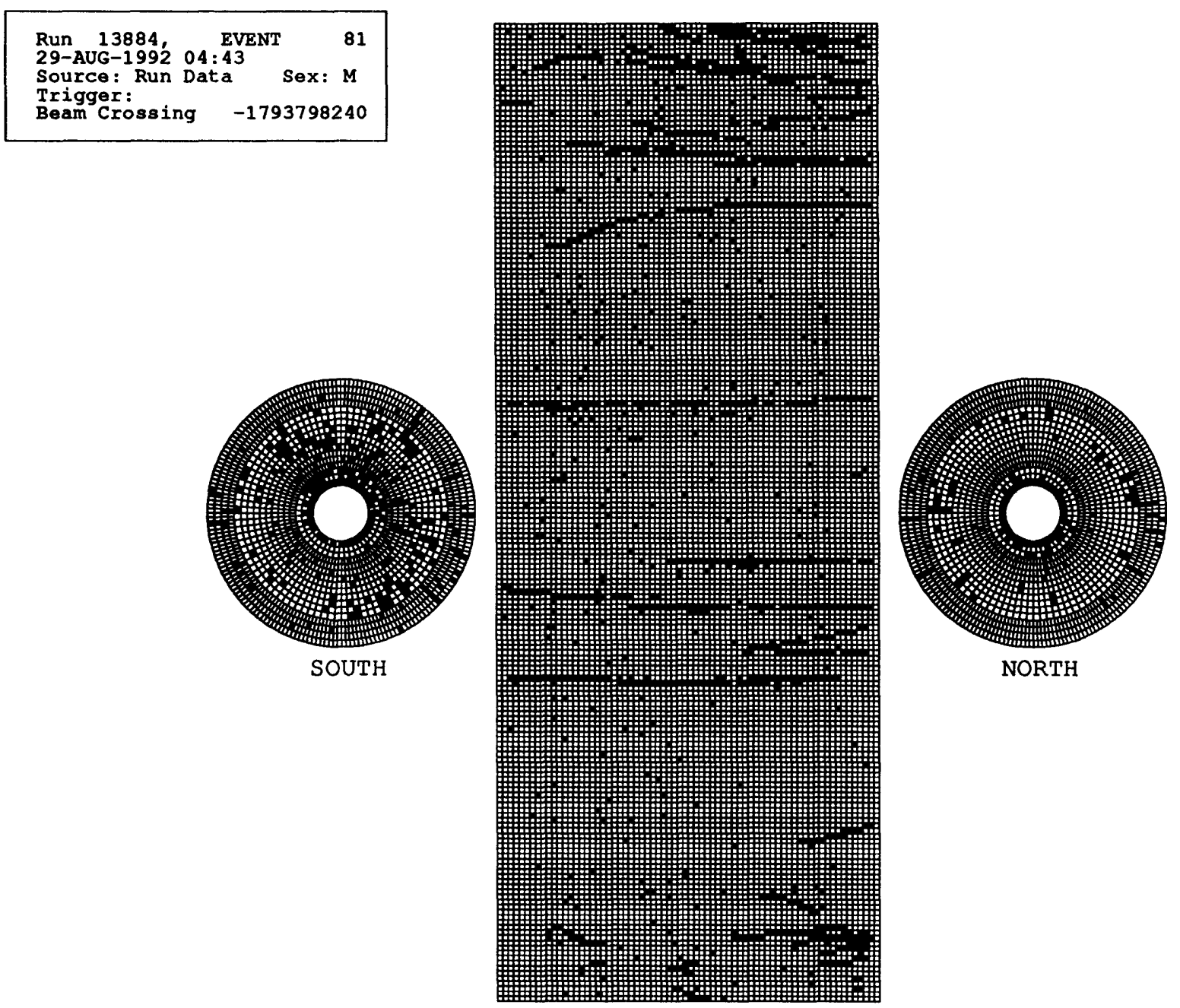

Figure 5-1: A typical background event triggered by upstream muons. In this display, the LAC towers with hits are displayed for the north and south endcaps and for a "rolledout" barrel. Note the distinctive signatures of the muons in the barrel and the "wall of fire" hits in the inner ring of the endcaps. 

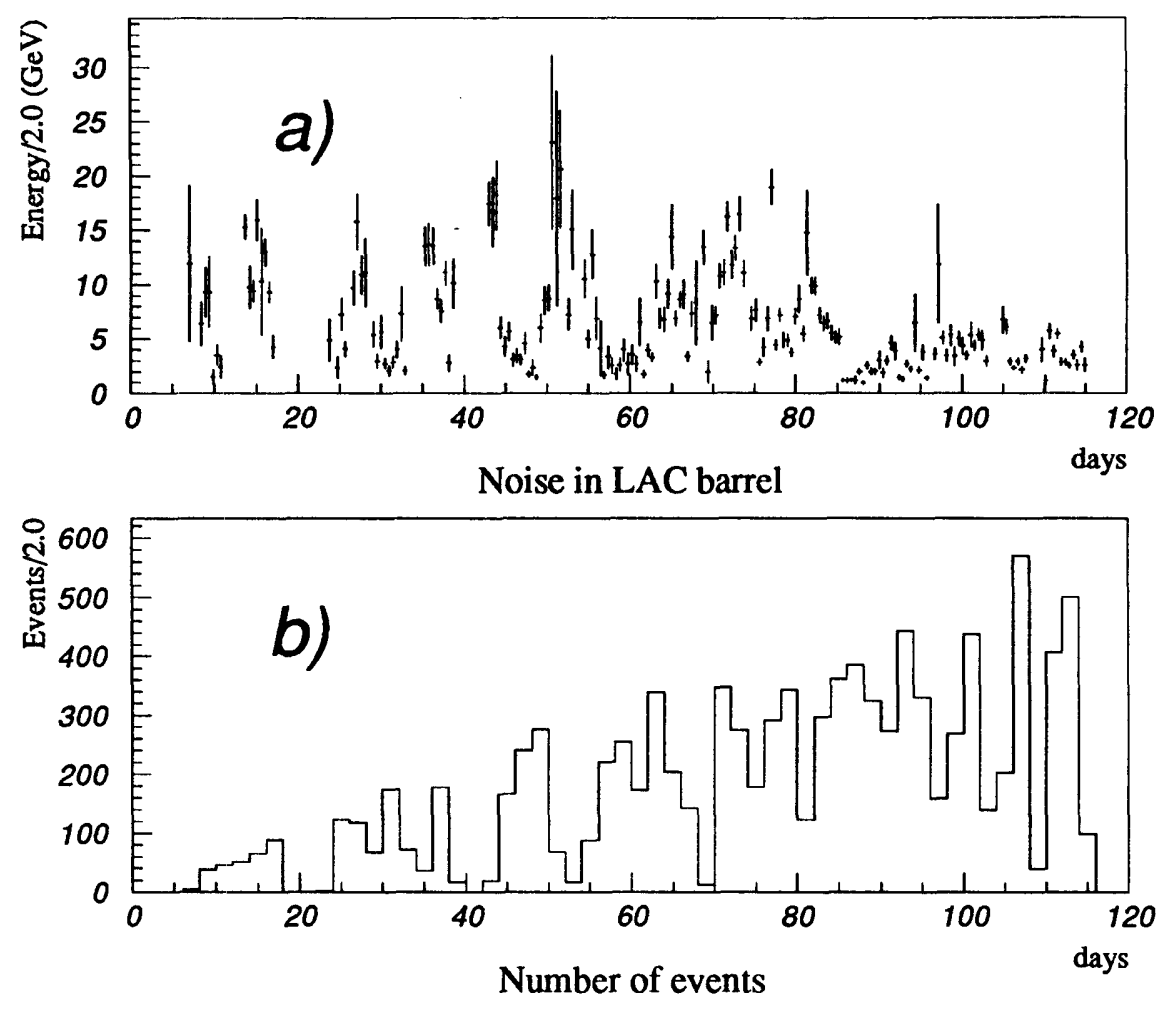

Figure 5-2: Time history of background muons in SLD. a) shows the the total energy carried by SLC-muon identified clusters in filtered events, and b) shows the number of selected hadronic events (see Section 5.5).

Since the wall-of-fire background was fairly localized and covered very little solid angle, the affected section of the detector could be simply ignored. In the rest of this analysis we will then zero the ADC counts for the two innermost towers in the LAC and the WIC both in the Monte Carlo and in the data. The net effect on the detector acceptance is minimal: the acceptance is reduced from $99.2 \%$ of $4 \pi$ to $98.8 \%$ of $4 \pi$ steradians.

The muons in the LAC barrel were easily pattern-recognized. An algorithm compared the $\theta$ and the $\phi$ spread of a cluster and tags clusters with many adjacent hits in $\theta$. Figure 5-2 shows the energy sum of the background-tagged clusters on an event by event basis. It is interesting to note the mild anticorrelation between luminosity and noise level - a phenomenon also observed online by the SLC operators. 


\subsection{Physics Backgrounds}

The Feynman diagrams for the physics processes which may fake a hadronic event in our data sample are illustrated in Figures 5-3a to 5-3d. At each stage of the event selection, we use a Monte Carlo simulation of these processes, together with the integrated luminosity determination of Section 5.1.1, to calculate the contamination to our hadronic sample. The references for the Monte Carlo generators used can be found in Section 3.4.

Of the processes in Figure 5-3, the $\tau^{+} \tau^{-}$decay mode is the one that contributes the most background for three reasons: $\tau$ 's tend to decay hadronically $(\approx 50 \%$ of the time [28]), they have a short lifetime $(c \tau=91.4 \mu \mathrm{m})$, and they have the same angular distribution as $q \bar{q}$ events. The $2 \gamma$ (Figure 5-3) process also has a hadronic mode, but as we will see, it is very 'soft'. The other processes in the figure have electromagnetic final states and are therefore easy to identify. We have seen in Chapter 3 that electromagnetic clusters are very 'tight' and do not deposit much energy in the hadronic calorimeter.

We will perform the same calculation for each of these backgrounds. As an illustration we will briefly go through the calculation for the $\tau^{+} \tau^{-}$channel here and will later quote the results for the other ones. We calculate the expected number of observed $\tau^{+} \tau^{-}$events for a given luminosity using,

$$
N_{\tau^{+} \tau^{-}}^{\text {expected }}=\epsilon_{\tau^{+} \tau^{-}}^{T} \rho_{E} \frac{\Gamma_{\tau^{+} \tau^{-}}}{\Gamma_{Z^{0}}} \sigma_{Z^{0}} L
$$

where $\Gamma_{Z^{0}}$ is the full width of the $Z^{0}, \Gamma_{\tau^{+} \tau^{-}}$is the width for $Z^{0} \rightarrow \tau^{+} \tau^{-}$, and $\sigma_{Z^{0}}$ is the total cross section. $\rho_{E}$ is a cross section dilution factor due to the beam energy spread. In addition,

$$
\epsilon_{\tau^{+} \tau^{-}}^{T}=\epsilon_{\tau^{+} \tau^{-}}^{\text {trigger }} \epsilon_{\tau^{+} \tau^{-}}^{\text {filter }} \epsilon_{\tau^{+} \tau^{-}}^{\text {selection }}
$$

is the total acceptance for $\tau^{+} \tau^{-}$pairs in terms of the efficiency for each of the selection stages and $L$ is the integrated run luminosity. Since the $\epsilon$ from above are derived from Monte Carlo simulations, they have systematic errors that depend on how well the Monte Carlo reproduces the data. In the case of this analysis, in which the overall cross section normalization is irrelevant, we may take the pragmatic approach and quote an effective $\epsilon^{T}$ (i.e. combined triggering, filtering, and selection) by direct comparison of the event yield with the integrated luminosity. We will take both approaches.

The above $\epsilon$ are calculated by generating Monte Carlo events of the particular process and calculating the fractional yield (i.e. $N_{\text {survive }} / N_{\text {thrown }}$ ) after simulating the trigger, the filter, and the selection. 

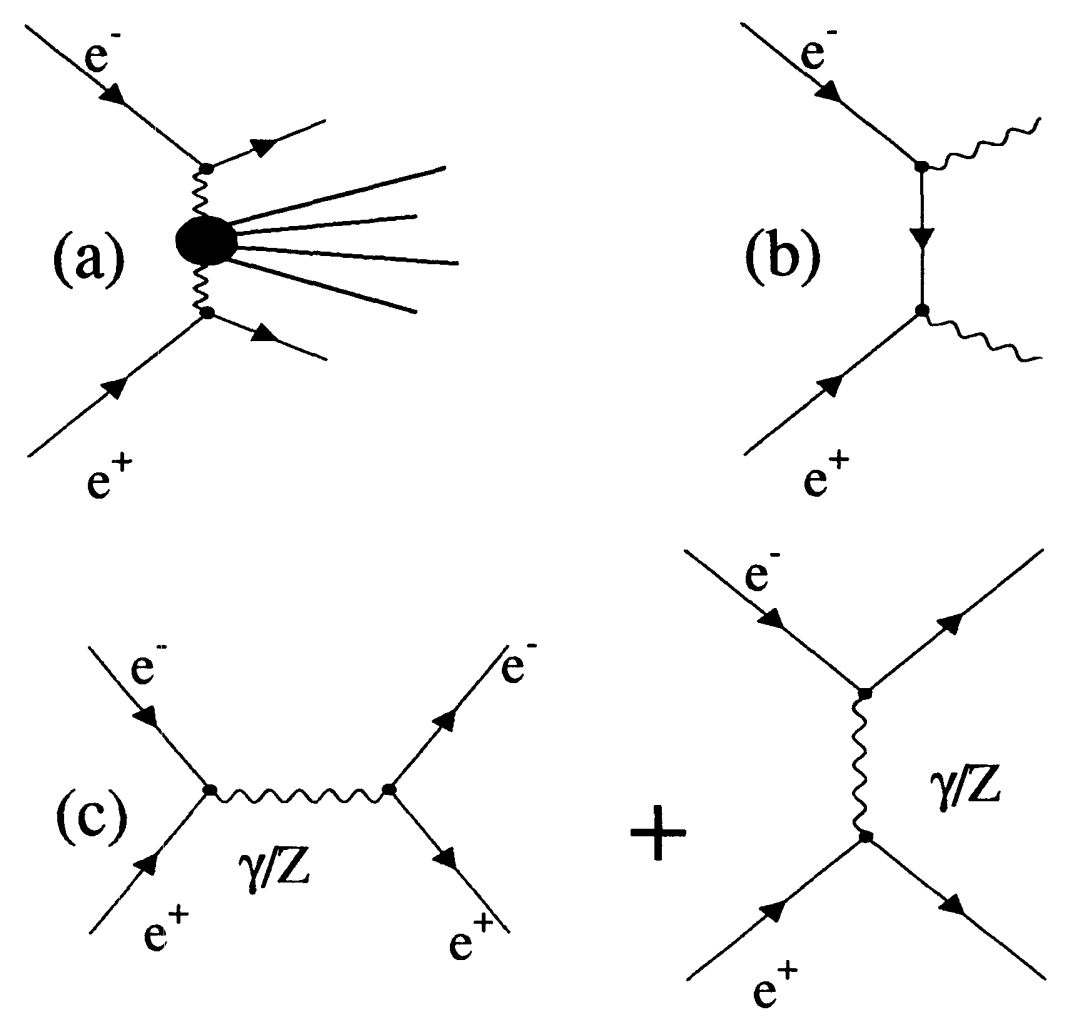

(d)

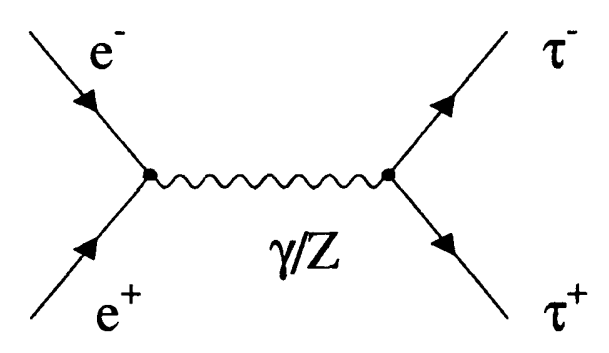

Figure 5-3: Backgrounds in the event selection: (a) the $2 \gamma$ process, (b) the pure QED $\gamma \gamma$ process, (c) the Bhabha process which can contaminate the hadronic sample in the forward region where there is limited acceptance, and (d) $\tau$ lepton final state whose hadronic decay mode can fake a $q \bar{q}$ state. 


\subsection{The Hadronic Event Trigger}

In Chapter 3 we have already reviewed all of the triggers used in the 1992 run. Here we describe the rationale for the calorimetry trigger since it is the relevant one for this analysis. The SLD calorimetry trigger is very simple. Hit energies (in ADC) above a certain energy threshold value are added into a single event energy sum. If the event energy sum is above the event energy threshold, the event is triggered by the acquisition system. We will now discuss the motivation for choosing these thresholds and energy sums.

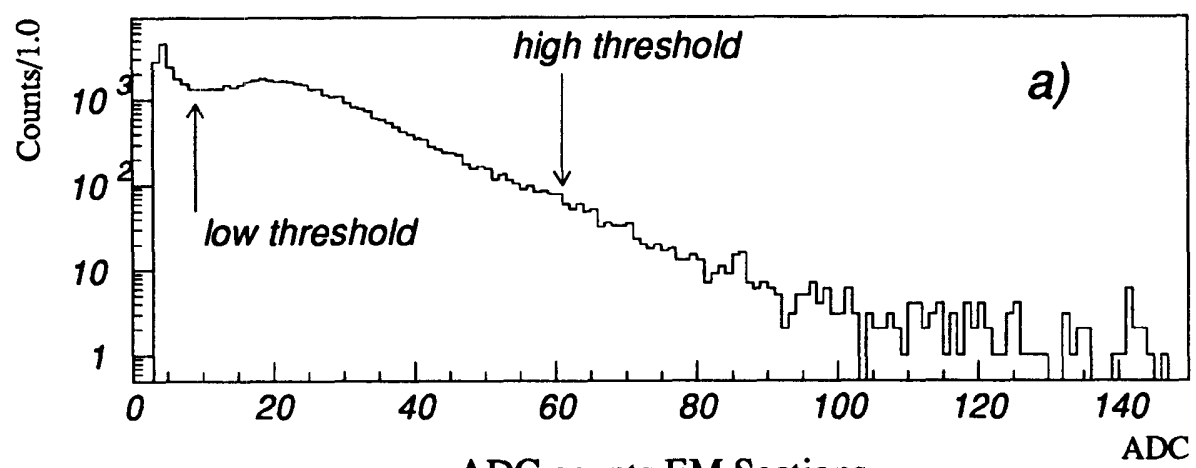

ADC counts EM Sections

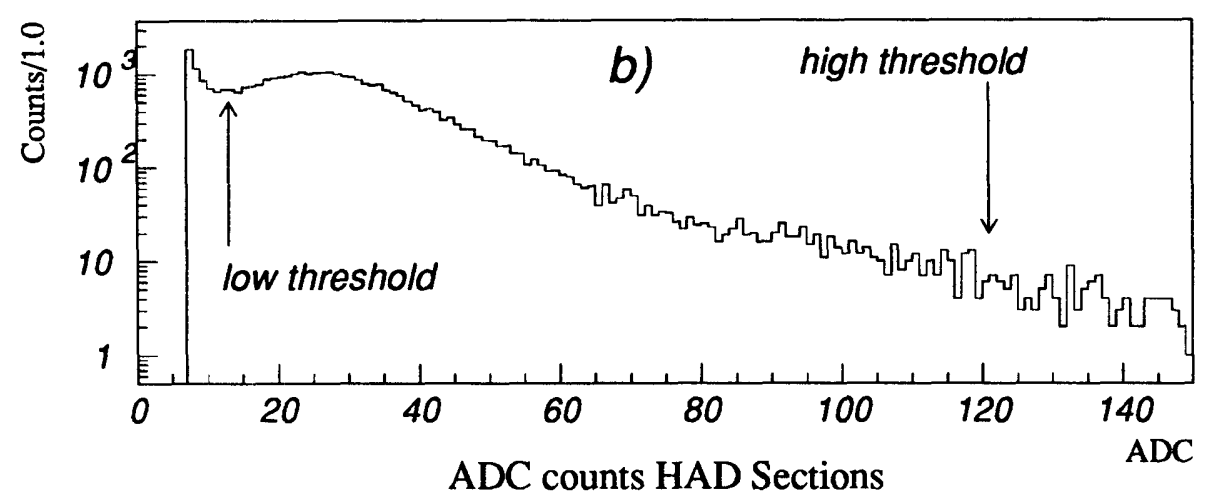

Figure 5-4: Hit spectra of muon backgrounds. Using luminosity events (Bhabha events in the luminosity monitor) to look at backgrounds provides a convenient luminosityweighted sample of minimum bias triggers in the central calorimeter. The spectra of the clusters identified as backgrounds is shown in a) for the electromagnetic section and in b) for the hadronic section. The arrows point to the dual trigger thresholds.

After reading the previous section it should be no surprise that one of the main aims in triggering was to reduce the SLC-induced backgrounds. After the 1991 Engineering Run, an SLD Trigger Task Force was formed to study all issues related to triggering, backgrounds, efficiency, and the dead-time introduced into the trigger by the trigger rates. One of the contributions of this Task Force was to implement a 


\begin{tabular}{||l||c|c|c||}
\hline \hline Layer $(l)$ & $c_{l}$ & $E_{H I}^{m i n, l}$ & $E_{L O}^{\min , l}$ \\
\hline \hline$e m-1$ and $e m-2$ & $524 \mathrm{MeV} / 128 \mathrm{ADC}$ & $60 \mathrm{ADC}$ & $8 \mathrm{ADC}$ \\
$h d-1$ and $h d-2$ & $1384 \mathrm{MeV} / 128 \mathrm{ADC}$ & $120 \mathrm{ADC}$ & $12 \mathrm{ADC}$ \\
\hline \hline
\end{tabular}

Table 5.1: Trigger quantities and their conversion factors.

trigger that was insensitive to the muon backgrounds and could simultaneously generate a noise signal to be relayed to the SLC operators. This signal was then to be used during the run to tune the machine orbits in order to reduce the backgrounds.

As can be seen from Figure 5-1, the SLC-induced muons leave very distinct patterns in the detector. The key to constructing a trigger which is insensitive to the background is then to exploit these patterns and energy depositions at the trigger level. The energy signatures of the SLC-related backgrounds are studied by looking at all clusters in the LAC in minimum bias events. Figure 5-4 shows the single-hit energy distribution of clusters present in the LAC in luminosity Bhabha events. The mip-like signature of the clusters is evident (compare with Figures 4-2 and 4-3). We will exploit this feature to reduce the trigger noise.

Typically there are 1-2 beam-related muons per beam crossing, each depositing $\approx 1 \mathrm{GeV}$ of energy in the LAC. If we take a conservative mean of $\left\langle n_{\mu}\right\rangle \approx 2$, the probability for a Poisson fluctuation in the total energy to reach the trigger energy is $\approx 10^{-3}$. With a beam crossing rate of $120 \mathrm{~Hz}$, this implies a muon induced trigger rate of at least $0.1 \mathrm{~Hz}$. This problem was minimized by [90] taking advantage of the mip spectrum shape. A hit threshold was chosen such that the SLC muon energy contribution to an event was minimal - essentially an ADC value well above the 'mip -bump'. This 'high-threshold' is shown with an arrow in Figure 5-4.

The SLD LAC hadronic event trigger consists then on a single energy sum over most of the detector region. The sum is formed with hits that have an energy higher than the high-threshold from Figure 5-4, $E_{H I}^{m i n}$; we can write this as:

$$
E_{H I}=\sum_{e_{i}^{l}>E_{H I}^{m i n, l}}^{n_{c e l l}} c_{l} e_{i}^{l}
$$

where $e_{i}^{l}$ is the individual hit energy in the $i$ th tower (in ADC counts), $l$ is the calorimeter layer index, and $n_{\text {cell }}$ is the total number of active towers in the detector. The $c_{l}$ are the sampling fraction ${ }^{1}$ correction factors. The correction factors $c_{l}$ and the chosen thresholds are given in Table 5.1. The chosen values minimize both beamrelated and instrumental noise.

An event triggers the acquisition system if $E_{H I}>8 \mathrm{GeV}$.

\footnotetext{
${ }^{1}$ See Chapter 3 . These factors correct for the difference in ratio of active to passive area in the different calorimeter layers.
} 
We have seen in Chapter 4 that energy fluctuations in the WIC are background dominated. In addition, we saw in Table 4.3 that on average the WIC receives less than $10 \%$ of the total event energy. It is then reasonable to exclude the WIC from the trigger calculation since the impact of the WIC on the trigger efficiency is minimal. In addition to ignoring the WIC, the 'wall-of-fire' towers, $\theta=47$ and $\theta=48$ are also excluded from the trigger sum.

The FASTBUS module that calculates the trigger at each beam crossing keeps a dual trigger sum. An analogue of $E_{H I}, E_{L O}$, is calculated with the lower thresholds presented in Table 5.1:

$$
E_{L O}=\sum_{e_{i}^{l}>E_{L O}^{m i n, l}}^{n_{\text {cell }}} c_{l} e_{i}^{l} .
$$

The quantity defined by Equation 5.4, by virtue of being on the low end of the 'mip -bump' is very sensitive to background muons. This quantity was delivered to the SLC operators as a real-time noise signal. We will also use it in the next section to help in the filtering stage.

The trigger module calculates another related quantity which we will also use in the next section: $N_{H I}^{e m}$. $N_{H I}^{e m}$ is defined as the number of hits in the LAC electromagnetic section (em-1 and em-2) above the ADC threshold $E_{H I}^{m i n}$. Since the electromagnetic section of the calorimeter 'sees' most of the event energy (See Table 4.3) and since the hadronic section is more sensitive to muon backgrounds (larger area), $N_{H I}^{e m}$ is a good discriminant against noise events.

\subsection{Event Filtering}

After the triggering stage, we are left with well over $1,100,000$ hadronic event candidates. Most of these events are background. Instead of proceeding to reconstruct all of the triggered data, and in order to reduce the total CPU time needed for processing, we filter out the background from the triggered sample. We take advantage of the available trigger quantities described in Section 5.3; by 'tightening' these quantities we are able to purify the data sample in favor of hadronic events.

The most powerful quantities in the filtering are $E_{H I}$ and $E_{L O}$. Since $E_{L O}$ is very sensitive to events with many low energy hits, it is a good discriminant against the SLC-muon events that conspire to pass the trigger. The power of this quantity is evident when the correlation of $E_{H I}$ and $E_{L O}$ is plotted for all events. Figure 5-5 shows this correlation with the filtering cuts represented by lines. There are 18,393 surviving events in the figure; originally (before filtering) there were well over 1 million events. Some features of this plot are worth noting. Monte Carlo simulations (discussed in more detail in the next section) show that the oval shaped cluster in the center of the figure consists mostly of hadronic events and some $\tau^{+} \tau^{-}$events. The diagonal stripe at high energy contains mostly Bhabha-like events. The horizontal stripe in the low end of $E_{H I}$ is due to SLC-muon events and a few $\tau^{+} \tau^{-}$events. The apparent 
diagonal cut is an artifact of $E_{H I} \geq E_{L O}$.

Figure 5-6 shows the cut applied to $N_{H I}^{e m}$. $N_{H I}^{e m}$ correlates very well with the total number of particles that strike the calorimeter. The cut $N_{H I}^{e m} \geq 10$ shown in the plot ensures that filtered events have hadronic-like multiplicities. The cuts used [84] to define the filtered sample (and shown in Figures 5-5 and 5-6) are:

- $N_{H I}^{e m} \geq 10$

- $E_{H I}>15 \mathrm{GeV}$

- $E_{H I}>\frac{3}{2} E_{L O}-105 \mathrm{GeV}$

- $E_{L O}<140 \mathrm{GeV}$

The energy units are $\mathrm{GeV}$ in the trigger scale presented in Table 5.1.

Following the recipe of Section 5.2 we can calculate the expected yields of different types of events for our filter. By using the Monte Carlo and a simulation of the filter we obtain the filter efficiencies summarized in Table 5.2. Figures 5-7 and 5-8 show the two key trigger quantities for both the data and the Monte Carlo. Using Figure 5-5 as a reference we can identify the regions of Monte Carlo-data discrepancy as the regions dominated by the SLC backgrounds. We also notice that $E_{H I}$ is only sensitive to these backgrounds in the low region as expected, while $E_{L O}$ is sensitive throughout. It should be noted that the $E_{H I}$ distribution for the data has entries below the minimum $15 \mathrm{GeV}$; the reason is that a small fraction of the events had the 'wall of fire' region included in the trigger - this produced an offset in the trigger distributions. These events will eventually be eliminated at the selection stage.

\subsection{Hadronic Event Selection}

Now that we have reduced the number of events to consider by a factor of 50 , we can fully reconstruct the remaining events. The SLD reconstruction has already been described in Section 3.5. From now on we will stop working with raw quantities and start using reconstructed clusters. Clusters are selected with the following requirements:

- a cluster energy of $E_{\text {clus }} \geq 150 \mathrm{MeV}$

- the electromagnetic correlation $G_{c} \geq 0.10$ ( $G_{c}$ is defined in Section 3.5.)

- $\left|\cos \theta_{\text {cluster }}\right| \leq 0.97$

- number of hits in a cluster $\geq 2$ 


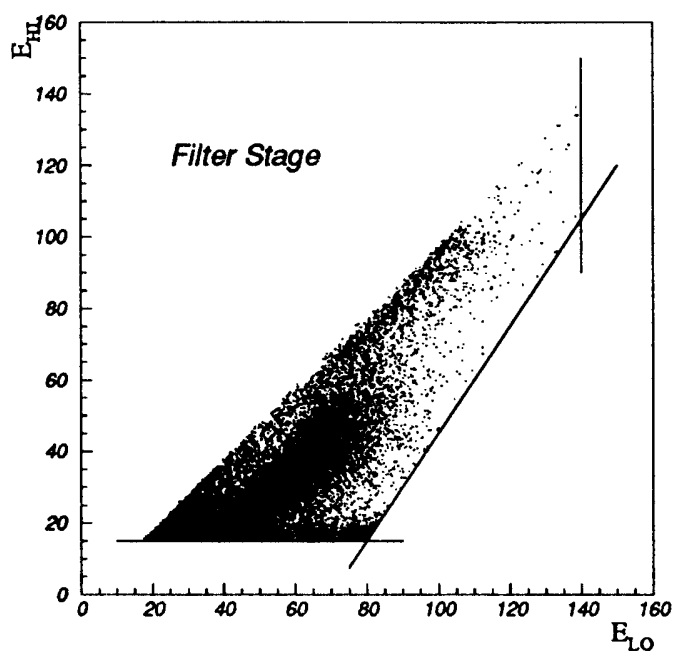

Figure 5-5: Plot of the trigger quantities $E_{H I}$ and $E_{L O}$ for the filtered events. The lines outline the cuts.

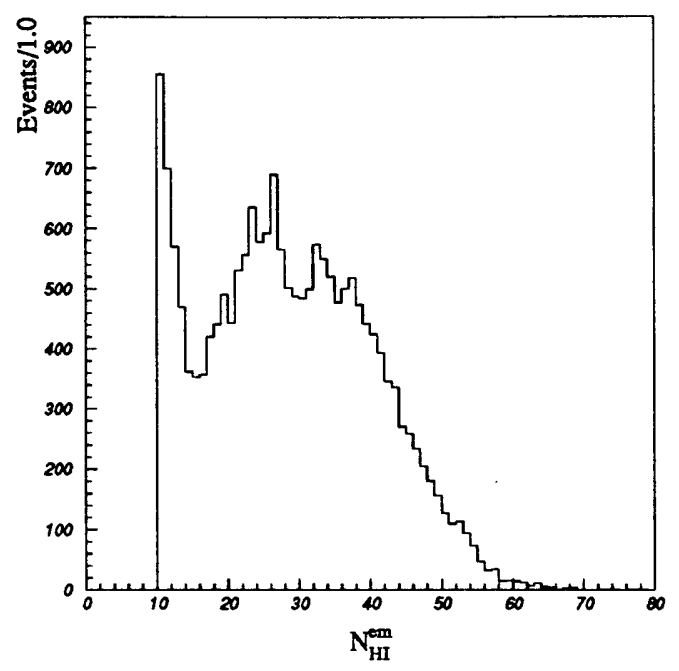

Figure 5-6: $\quad$ Plot of $N_{H I}^{e m}$ for the filtered events. The rise due to background is evident at low $N$.

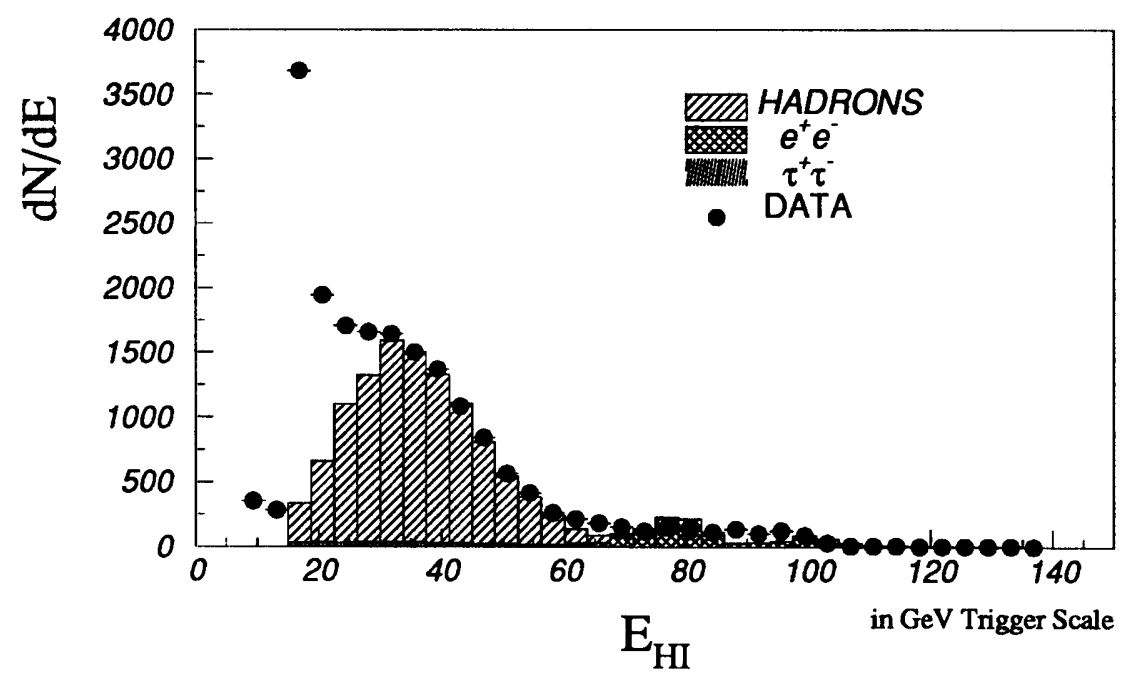

Figure 5-7: $\quad E_{H I}$ for filtered data and Monte Carlo with backgrounds. The yield for Monte Carlo hadrons and backgrounds was calculated using the 1992 integrated luminosity.

These cuts reduce spurious noise and select those clusters with energy deposition in the electromagnetic section of the calorimeter. We will treat these selected clusters as pseudo-particles. This approach will enable us to exploit the characteristics of the different physics processes (i.e. $q \bar{q}$ events + background) in order to be able to differentiate between them. 


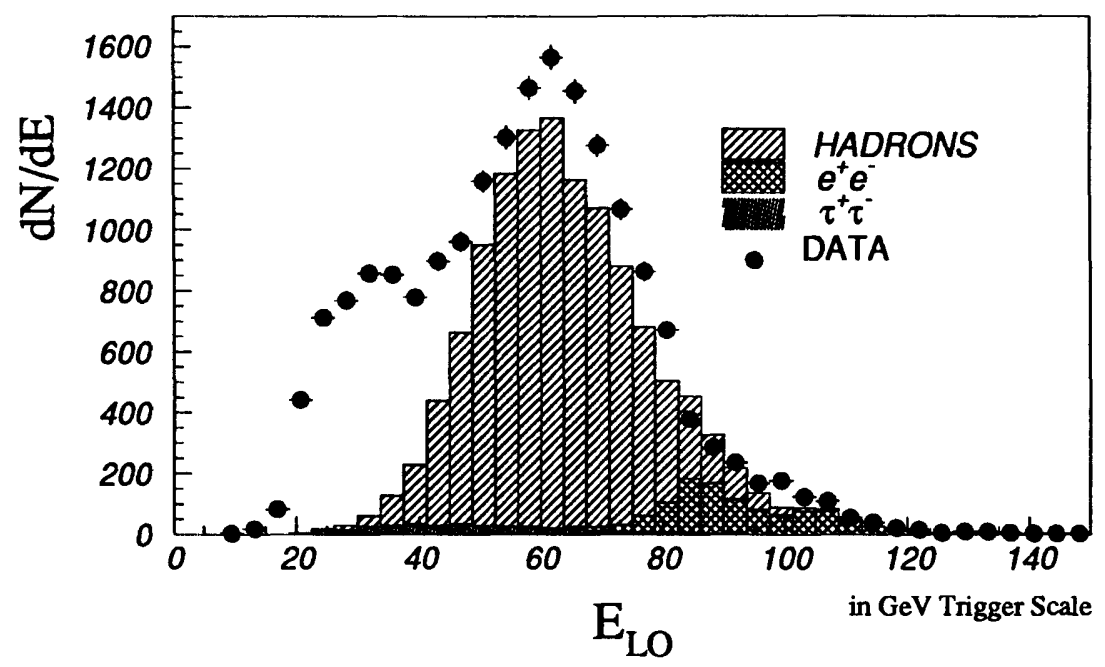

Figure 5-8: $\quad E_{L O}$ for filtered data and Monte Carlo with backgrounds. The excess at low energies is due to the un-modelled SLC induced backgrounds.

We will now discuss the global event quantities used in the event selection. Unless otherwise noted, the quoted errors in the efficiencies will be both statistical and systematic. Systematic errors are estimated by varying the energy scale. In the case of $q \bar{q}$ events, systematic errors also include contributions from the difference of the two Monte Carlo generators used, JETSET [45] and HERWIG [50].

\section{Energy Imbalance}

We define the energy imbalance, $E_{i m b}$ as,

$$
E_{i m b}=\frac{1}{E_{v i s}}\left|\sum_{i=1}^{N_{c l u s}} E_{i} \mathbf{n}_{i}\right|
$$

where $\mathbf{n}_{i}$ is a unit vector pointing from the IP to the centroid of the $i$ th cluster (See section 3.5) and $E_{v i s}$ is the event visible energy defined below. The energy imbalance measures the magnitude of the missing energy vector. By vectorizing the energy clusters we take advantage of the fact that, barring resolution, invisible particles ${ }^{2}$, and acceptance effects, the vectored-energy sum should be zero for a physics event. This cut therefore is most effective in reducing beam-related backgrounds. Figure 5-9 shows the energy imbalance for the filtered sample and for the various physics processes (Monte Carlo). The chosen cut of $E_{i m b} \leq 0.65$ has an efficiency of $98.4 \pm 0.1 \pm 0.5 \%$.

\footnotetext{
2'Invisible particles' are those that deposit little or no energy in the calorimeter. Neutrinos almost never interact and muons deposit very little energy (remember mip scale from Chapter 4) independent of their momentum.
} 


\section{Number of Clusters}

Hadronic events at the $Z^{0}$ are characterized by multiplicities of $\approx 20$ charged particles [91]. Neutral particles contribute ${ }^{3}$ an additional $\approx 20$ clusters per event, mostly from the decay $\pi^{0} \rightarrow \gamma \gamma\left(\left\langle n_{\pi^{0}}\right\rangle=10\right.$ [92]). Our main sources of physics backgrounds, $e^{+} e^{-}$and $\tau^{+} \tau^{-}$events, have very low multiplicities. Figure 5-10 shows the $N_{\text {clus }}$ distribution for the filtered data and the Monte Carlo with backgrounds. The cut shown in the plot (arrow), $N_{\text {clus }} \geq 9$, has an efficiency of $97.4 \pm 0.2 \pm 1.0 \%$.

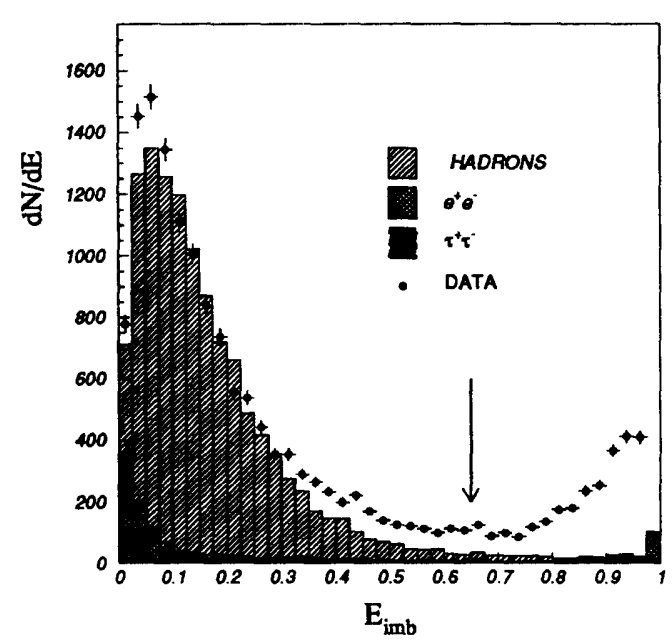

Figure 5-9: Plot of the normalized energy imbalance, $E_{i m b}$, for the filtered events. The arrow points to the chosen cut.

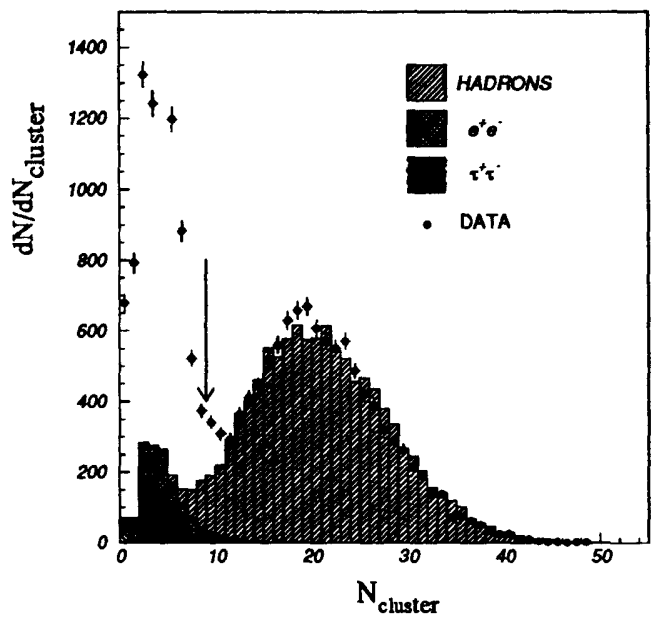

Figure 5-10: $\quad$ Plot of $N_{\text {clus }}$ for the filtered events. The rise due to background is evident at low $N$.

\section{Visible Energy}

The total energy in an event provides a distinctive signature for hadronic events. In Chapter 4 we saw that most of the energy deposited in the calorimeter in a $q \bar{q}$ event is hadronic in nature (i.e, the ionization is due to hadronic inelastic scattering). We also saw that since the intrinsic $\frac{e}{\pi}$ of the calorimeter was $\approx 1.7$, the detector response to electrons was markedly higher than to hadrons. This makes $e^{+} e^{-}$events easy to eliminate by visible energy alone (high $E_{v i s}$ ). The $\tau^{+} \tau^{-}$events also have a distinctive $E_{v i s}$ signature. They tend to have low total energies since a large fraction of their final state [28] is composed of invisible particles (neutrinos and muons). Figure 5-11 shows the $E_{v i s}$ distribution for both data and Monte Carlo. Backgrounds are also

\footnotetext{
${ }^{3}$ The charged/neutral multiplicities that a calorimeter 'sees' depends in great part in the cluster minimum energy cutoff used. For instance, charged particles with a transverse momentum of $p_{t} \lesssim 200 \mathrm{MeV}$ will not reach the barrel calorimeter because of the magnetic field bending and $d E / d X$ energy loss while neutral particles have no such limitations.
} 
included. The excess in the data (see figure) is due to the unmodelled beam-related backgrounds. A cut in the visible energy, $E_{v i s}$, of:

$$
\begin{cases}0.50 \leq E_{v i s} / E_{c m} \leq 1.5 & |\cos \theta| \leq 0.8 \\ 0.35 \leq E_{v i s} / E_{c m} \leq 1.1 & |\cos \theta|>0.8\end{cases}
$$

has an efficiency of $97.7 \pm 0.1 \pm 0.4 \%$. The same distribution (normalized to $E_{c m}$ ) but separated into endcap and barrel regions is shown in Figure 5-12.

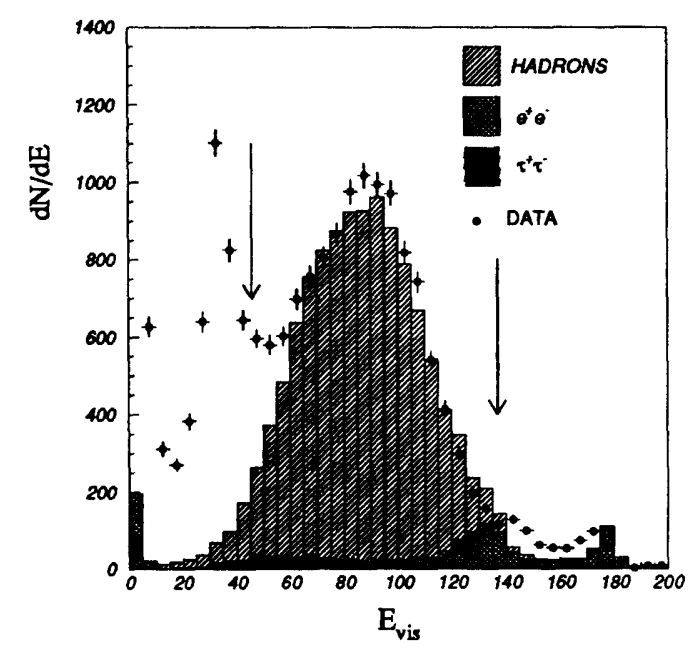

Figure 5-11: Visible Energy for filtered events.
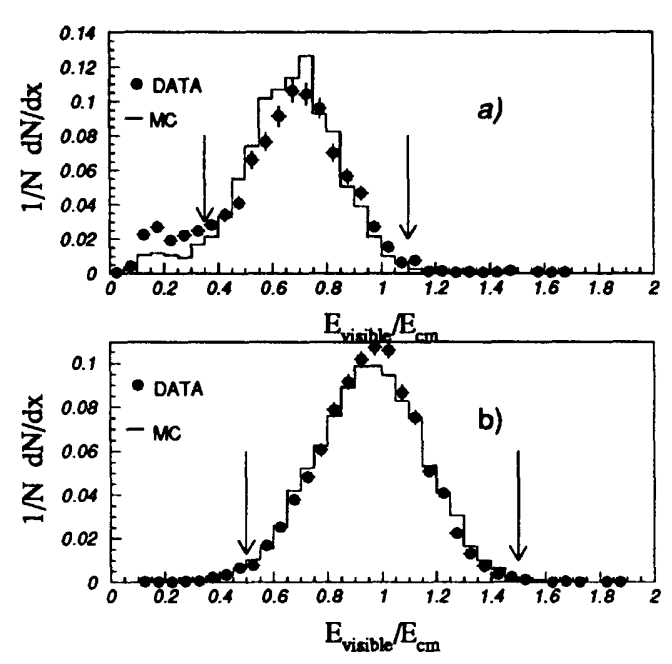

Figure 5-12: $\quad E_{\text {vis }}$ for pre-selected events: a) EndCap and b) Barrel.

\section{Polar dependence of $E_{v i s}$}

Figure 5-13 presents the visible energy as a function of the angle of the event thrust axis ${ }^{4}$ with the beamline for filtered events. Thus $\left|\cos \theta_{\text {thrust }}\right|=1$ corresponds to an event oriented perpendicular to the endcaps. The energy imbalance cut of $E_{i m b} \leq 0.65$ has been applied to eliminate most of the non-physics backgrounds. Two features stand out about the figure. The first feature is the two distinct energy bands across the detector. The upper band is due to $e^{+} e^{-}$events and the lower band is due to the bulk of the $q \bar{q}$ events - demonstrating again the unevenness of the electromagnetic and the hadronic response. The second feature is the degradation in response in the endcap region. One sees that the response, both for electrons and hadrons, is uniform in the barrel region $|\cos \theta| \lesssim 0.65$. The region $0.65 \lesssim|\cos \theta| \lesssim 0.8$ is the overlap region (refer to Figure 3-3) and a lower response is expected due to acceptance effects. The endcap region, $|\cos \theta| \gtrsim 0.8$, has a markedly degraded response in terms of visible

\footnotetext{
${ }^{4}$ For a definition of thrust, see Appendix A.
} 
energy and resolution. This initially unexpected behavior was hypothesized ${ }^{5}$ to be a result of extra material in the endcap region; by adding material in the Monte Carlo and correcting the accounting of the geometry, this interpretation was later shown [93] to be correct.

\subsection{Results}

The efficiencies for selecting hadronic events, and their backgrounds, are presented in Table 5.2. After triggering, filtering, and applying the selection cuts described above to the 1992 run data sample, we are left with 9,878 events. If we contain our event sample to the fiducial volume of $\left|\cos \theta_{t h r}\right| \leq 0.92$ in order to minimize leakage into the beampipe, then our sample is reduced to 9,143 events. The results of these two selections are shown in Tables 5.2 and 5.3. Table 5.3 contains the estimates of background present in our selected hadronic sample. Since the $\gamma \gamma$ process looks like the Bhabha process in the calorimeter (except for the angular distribution), we quote a conservative estimate of $\gamma \gamma$ contamination by multiplying the $e^{+} e^{-}$selection efficiency with a Monte Carlo calculated [94] yield of 0.7 events for the entire run. No background is above the level of a fraction of a percent.

\section{Luminosity Predicted Yields}

We can use equations 5.1 and 5.2 to calculate the expected number of hadronic events given the cross section, the measured luminosity, and the calculated efficiencies:

$$
N_{e x p e c t e d}^{q \bar{q}}=L \epsilon_{q \bar{q}}^{T} \sigma_{q \bar{q}} \rho_{E}
$$

Using $\rho_{E}=0.97 \pm 0.01$ for the cross section dilution factor due to beam spread [95] and using $\sigma_{q \bar{q}}=29.5 \mathrm{nb}$, we obtain $N_{\text {expected }}^{q \bar{q}}=10,080 \pm 150$ events. The error is a combination of both statistical and systematic errors for the efficiency (calculated from the Monte Carlo) and the error on $\rho_{E}$. The expected number of events is in agreement with our yield. The agreement holds for both fiducial regions.

\section{An Event}

Three views of a selected hadronic event are shown in Figure 5-14. In this particular display, the energy hits are represented by squares whose area is proportional to the measured energy. The long tower-like cones are the clustered hits. It is events like

\footnotetext{
${ }^{5}$ Since electromagnetic showers scale like $X_{0}$ and hadronic showers like $\lambda_{i n t}$, electrons are more affected by the pre-showering induced by 'unknown' material. The fact that the resolution was much more degraded for electrons than for hadrons in the endcap was a strong hint of the origin of the problem. In addition the mip response was identical for the endcap and the barrel; this implies that the intrinsic ionization response for both subsystems is the same.
} 
these that provide striking evidence for hard gluon radiation. We will investigate these issues in the next Chapter.

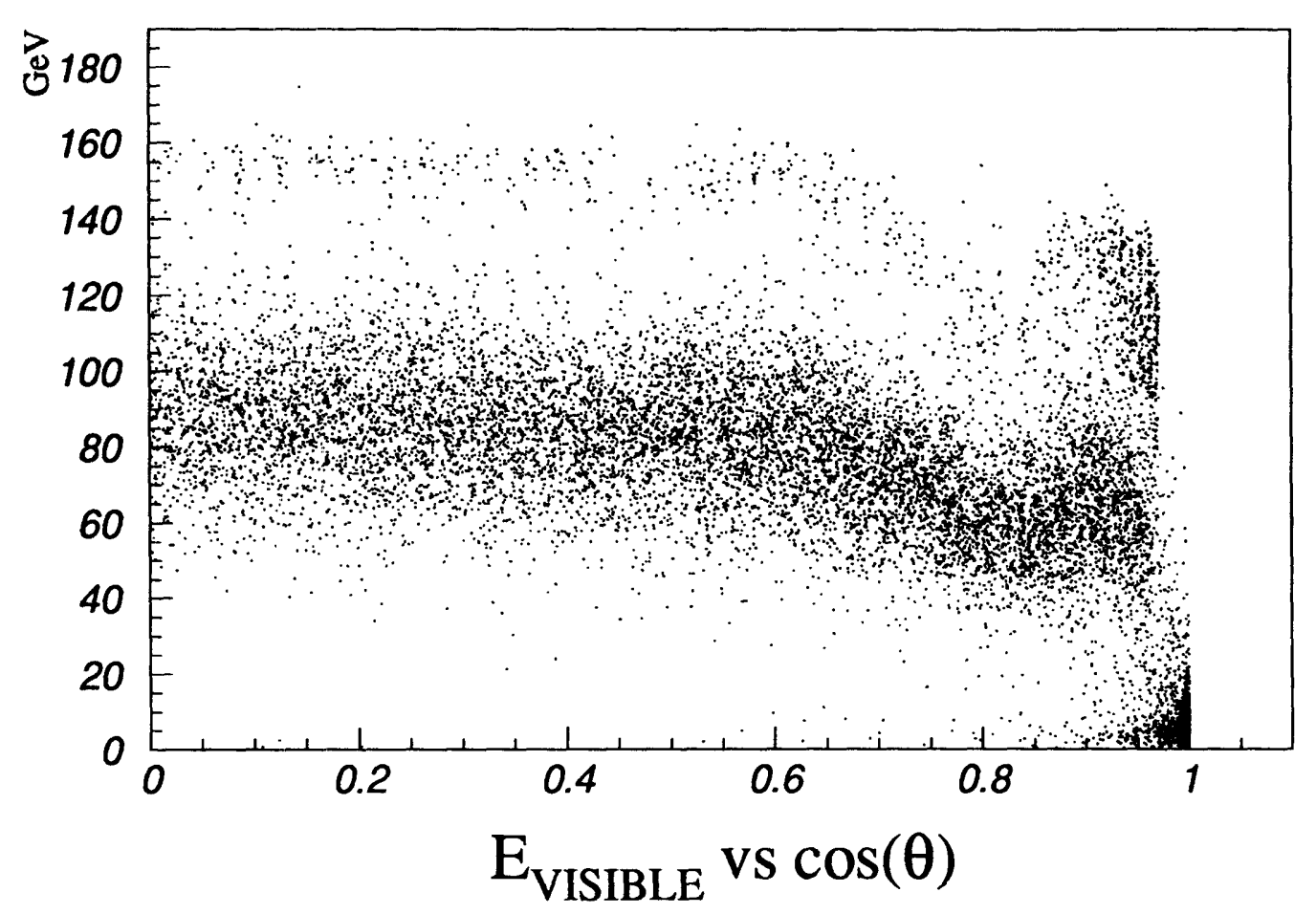

Figure 5-13: $\quad E_{v i s}$ vs $\cos \theta_{t h r u s t}$ for filtered events with an imbalance cut of $E_{i m b} \leq 0.65$. Energy is in GeV. Notice the narrow $e^{+} e^{-}$energy band above the wider $q \bar{q}$ energy band. The degraded endcap $(|\cos \theta| \gtrsim 0.8)$ response is also evident.

\begin{tabular}{||l|c|c|c|c||}
\hline \hline Process & $\epsilon_{\text {trigger }}$ & $\epsilon_{\text {filter }}$ & $\epsilon_{\text {selection }}^{1}$ & $\epsilon_{\text {selection }}^{2}$ \\
\hline \hline$q \bar{q}$ & $99.4 \pm 0.1 \pm 0.3$ & $97.6 \pm 0.2 \pm 0.8$ & $94.3 \pm 0.2 \pm 0.2$ & $86.8 \pm 0.3 \pm 0.2$ \\
$\tau^{+} \tau^{-}$ & $86.4 \pm 0.8 \pm 1.6$ & $75.5 \pm 1.3 \pm 2.0$ & $6.3 \pm 0.8 \pm 0.8$ & $5.7 \pm 0.7 \pm 0.7$ \\
$e^{+} e^{-}$ & $99.9 \pm 0.1 \pm 0.1$ & $99.5 \pm 0.2 \pm 0.5$ & $0.7 \pm 0.2 \pm 0.1$ & $0.7 \pm 0.2 \pm 0.1$ \\
$2 \gamma$ & $1.9 \pm 0.2 \pm 0.3$ & $16.2 \pm 4.3 \pm 4.0$ & $0.2 \pm 0.1 \pm 0.1$ & $<0.2 @ 90 \%$ C.L. \\
\hline \hline
\end{tabular}

Table 5.2: Yields for the different stages of event selection in percentage. The errors quoted are statistical and systematic respectively. The systematic errors were calculated from a $7 \%$ variation in the energy scale and the number of clusters. 


\begin{tabular}{||l|c||c|c||}
\hline \hline Process & \multicolumn{3}{|c||}{ Contamination } \\
& $\sigma_{\text {eff }}$ & $\left|\cos \theta_{\text {thr }}\right| \leq 0.92$ & $\left|\cos \theta_{\text {thr }}\right| \leq 1.0$ \\
\hline \hline$\tau^{+} \tau^{-}$ & $1.4 \mathrm{nb}$ & $0.18 \pm 0.05$ & $0.20 \pm 0.05$ \\
$e^{+} e^{-}$ & $4.3 \mathrm{nb}$ & $0.10 \pm 0.05$ & $0.10 \pm 0.05$ \\
$2 \gamma$ & $5.2 \mathrm{nb}$ & $\leq 0.02 @ 90 \%$ C.L. & $\leq 0.04$ @ 90\% C.L. \\
$\gamma \gamma$ & - & $\lesssim 0.02 @ 90 \%$ C.L. & $\lesssim 0.02$ @ 90\% C.L. \\
\hline \hline
\end{tabular}

Table 5.3: Summary of backgrounds in percent of hadronic sample. The quoted cross section is the effective cross section into the calorimeter fiducial volume.
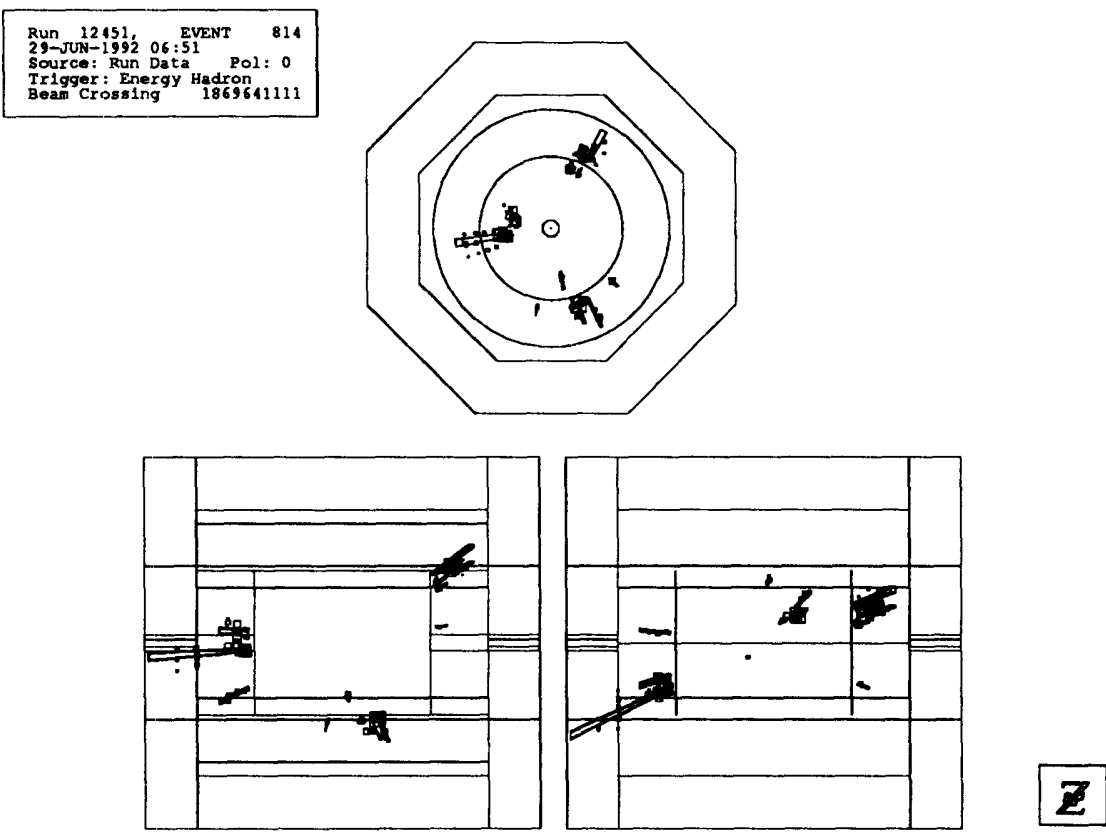

Figure 5-14: Three views of a 3 -jet event at $y_{c}=0.17$ in the JADE recombination scheme. The top figure is looking into the beampipe, the bottom-left is east to west, and the bottom-right is top to bottom. 


\section{Chapter 6}

\section{The Measurement}

\subsection{Introduction}

In this chapter we proceed to measure the value of $\alpha_{s}$ near the $Z^{0}$ sector by measuring the rate of gluon radiation from the primary quark/anti-quark pair. We will start by describing the experimental observable (jet-rates) and the detector effects on its measurement. We will study the resolution of the experimental quantities and develop methods to correct for the detector effects. At that point we will draw heavily on our knowledge of the SLD calorimeter system described in Chapters 3 and 4.

After correcting the jet rates for detector effects we will apply further corrections that will enable us to perform a direct fit for the QCD parameter $\Lambda_{\overline{M S}}$. These corrections introduce significant theoretical systematic uncertainties; we will discuss those. This discussion will be based on the framework presented in Chapter 2 on perturbative and non-perturbative QCD.

\subsection{Jets in the SLD Calorimeter}

We have seen in Chapter 2 that jets are a rich manifestation of QCD in the energy scale range $\Lambda_{Q C D}^{2} \lesssim Q^{2} \lesssim E_{c m}^{2}$, where $E_{c m}$ is the center of mass energy of the process in question. In the limit of high- $Q^{2}$ we expect the observed jets to correlate with the initially produced partons. In addition, in this limit, perturbative calculations to second order in $\alpha_{s}$ are available which enable us to test QCD at the perturbative scale. However, as we also discussed in Chapter 2, these partons cannot be observed directly. What we do observe are the hadrons left over by the fragmentation process.

In order to understand the methodology of measuring jets it is instructive to consider PQCD to $\mathcal{O}\left(\alpha_{s}\right)$. In this case, we have the tree level graphs contributing to $e^{+} e^{-} \rightarrow q \bar{q}, q \bar{q} g$ and the associated vertex and loop corrections (See Figure 23) to $\mathcal{O}\left(\alpha_{s}\right)$. However, even if we were able to measure the partons' direction and energy directly, we would still encounter (when comparing to the cross section in 


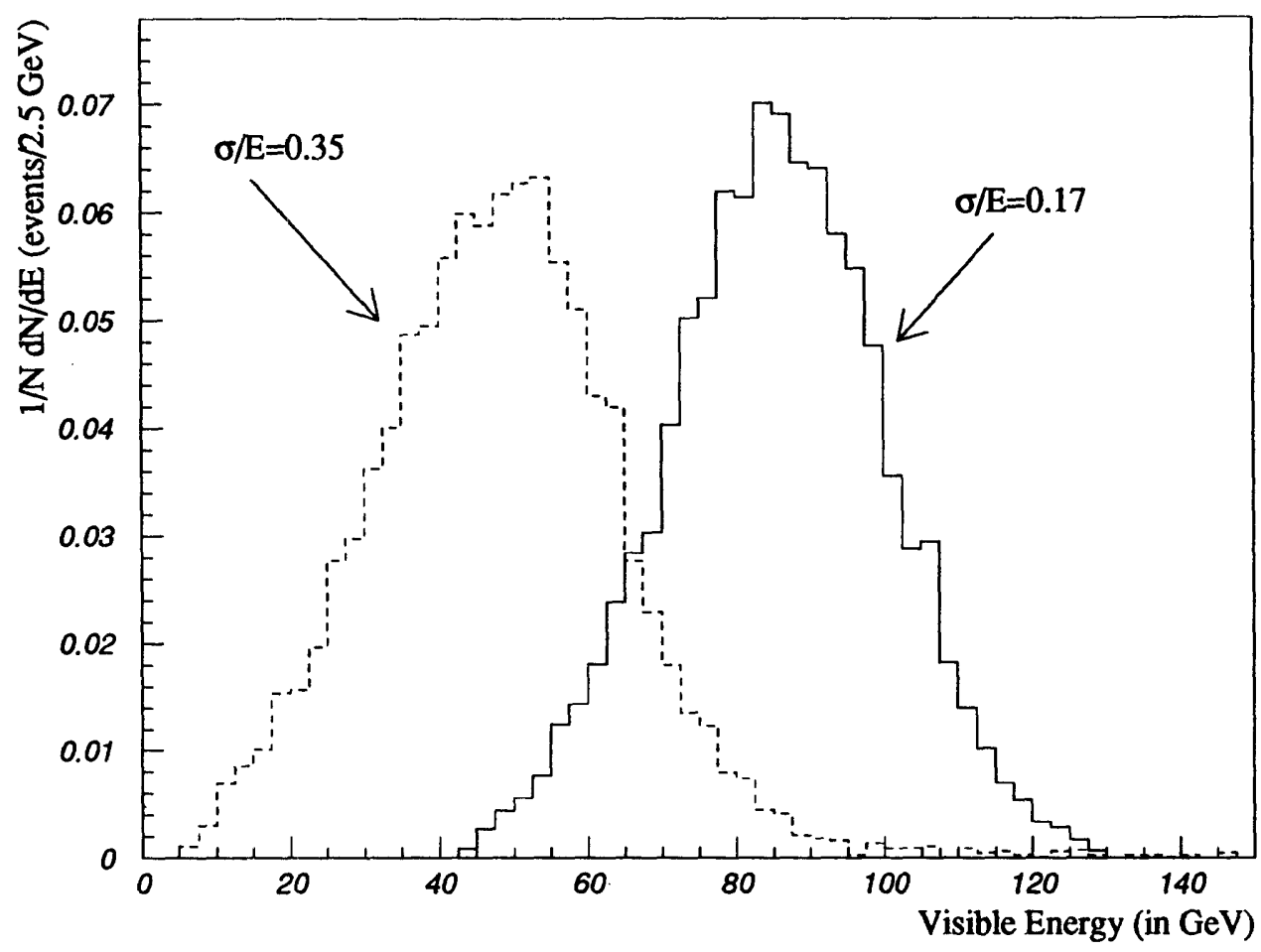

Figure 6-1: Comparison of the total energy distribution for tracking and calorimetry in the SLD data. Only events with $\left|\cos \theta_{\text {thrust }}\right| \leq 0.65$ are included. The solid line is the calorimeter distribution and the dashed line is the tracking distribution. The tracking distribution was obtained from reference [66].

Equation 2.18) the infrared and collinear singularities associated with $x_{g} \rightarrow 0$. This situation is to be expected since as $x_{g} \rightarrow 0$ we start to probe the point-like nature of QCD and the associated singularities discussed in Chapter 2.

Fortunately, though, we have a natural experimental cut-off. The finite resolution of the calorimeter and the imposed energy thresholds explicitly impose a cut-off on the soft radiation that we can distinguish experimentally. We thus need not be concerned with the soft and collinear region of phase space when using the PQCD calculations; we simply average over such final states since they are indistinguishable.

We mentioned in Chapter 1 that a calorimeter exhibits the good-PQCD property of collinear and infrared safety when measuring clusters of energy. This fact, and the natural jet definition which we will adopt, will allow us to treat calorimeter clusters as pseudo-particles. These clusters are the result of energy-hit merging (by the finite calorimeter granularity) or splitting (by acceptance or efficiency effects) of the impinging particles in the detector. Thus, we need not worry whether cluster $i$ is really composed of particles $j$ and $k$ or just of a particle $i$. As long as we understand 
how the detector does this merging and splitting we can correct for it with Monte Carlo simulations. We will discuss this aspect in the next section.

Due to various factors, including cost, tolerance to background, and instrumentation limitations, calorimeters in $e^{+} e^{-}$colliders are, in general, more hermetic than tracking devices. This, of course, implies the good acceptance which we have also discussed. This good hermiticity and the charge-blindness that calorimeters enjoy translate into a better overall resolution than a tracking device. This result is shown in Figure 6-1 for the SLD detector with the 1992 data. From the figure, it is clear that for a global quantity like $E_{v i s}$ the calorimeter resolution is at least one-half of the drift chamber resolution. A calorimeter is therefore a natural place to measure the rate of gluon radiation.
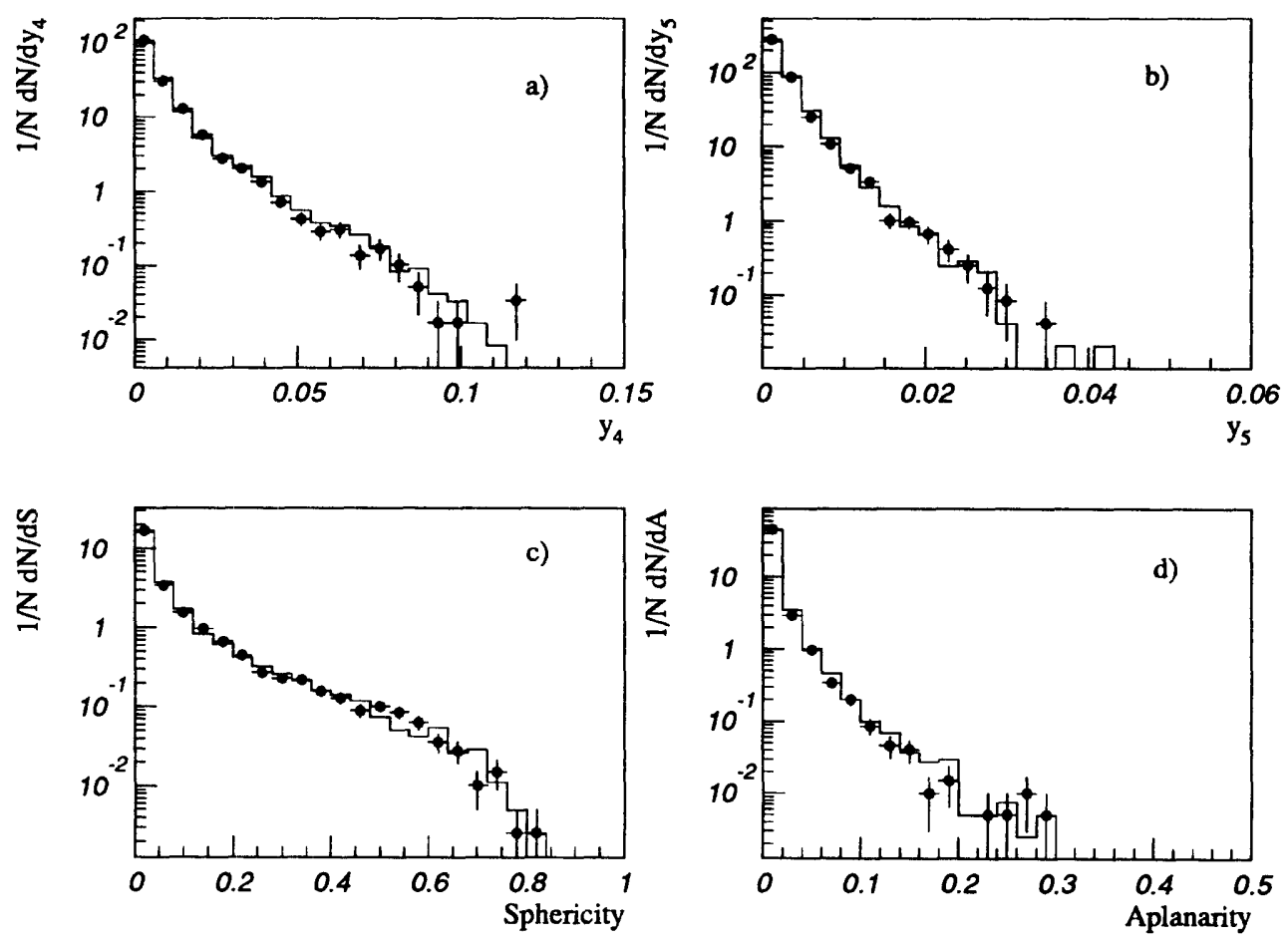

Figure 6-2: Four QCD observables in the data (dots) and in the JETSET 6.3 PS Monte Carlo (line). These quantities are defined in Appendix A: a) $y_{4}$, b) $y_{5}$, c) Sphericity, and d) Aplanarity. This good agreement between the data and the Monte Carlo is crucial to demonstrate the validity of the correction procedure. 


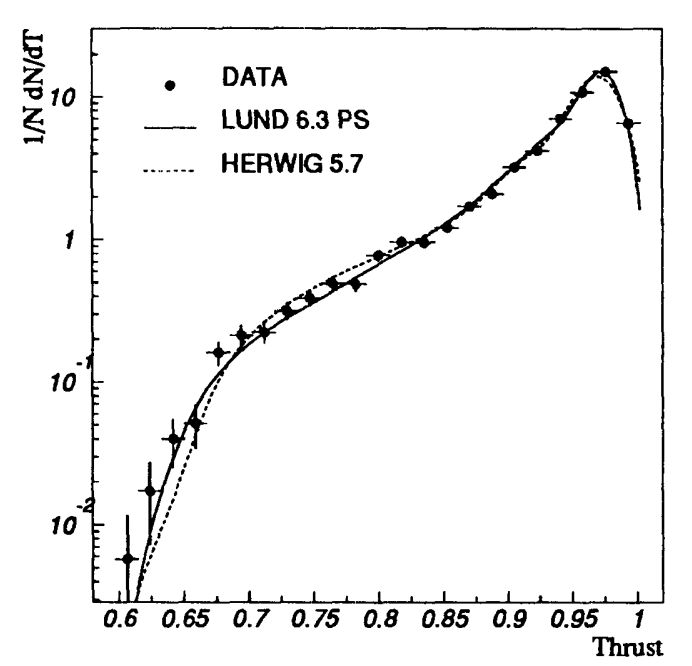

Figure 6-3: The thrust distribution for observed data. The errors shown are statistical only. The Monte Carlo distributions shown include a simulation of the detector and of initial state radiation.

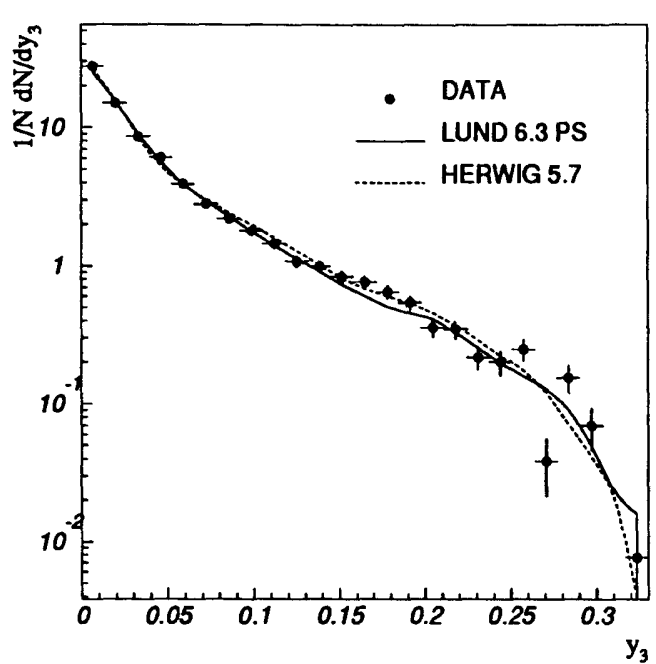

Figure 6-4: $\quad$ The distribution of $y_{3}$ (see text) for the observed data. The errors shown are statistical only. The Monte Carlo distributions included in the figure include a simulation of initial state radiation and detector effects.

\section{Comment on Data-Monte Carlo Agreement}

Throughout this work we have stressed the importance of the ability of the detector simulation to reproduce the data. We will soon see that this condition is essential for our analysis since the Monte Carlo is the only tool we have to unfold the detector effects from the measurement.

After the Monte Carlo tuning and detector calibration of Chapter 4, the only way to check the veracity of the detector simulation is to compare physics distributions for the data and the Monte Carlo. Figure 6-2 shows such a comparison between the data and the JETSET 6.3 PS Monte Carlo. Figures 6-3 and 6-4 show the thrust and $y_{3}$ distributions respectively for the data and the JETSET and HERWIG Monte Carlos. These two observables are particularly sensitive to the QCD physics; the data-Monte Carlo agreement is again very good.

At this point one may be tempted to argue that any measurement that makes heavy use of the Monte Carlo (as is our case) is inherently suspect and biased to the Monte Carlo input parameters. This very important issue is addressed in Appendix B, where we show, using a "toy model", that this is not the case. 


\subsubsection{Definition of Jets and Jet Algorithms}

One of the most important issues when choosing how to define a jet is the applicability of the chosen jet algorithm at all levels of the parton $\rightarrow$ hadron decay chain. This requirement ensures a quantitatively consistent definition of a jet at both the theoretical and the experimental levels. Another desirable characteristic is that the jet definition, in order to be calculable in $\mathcal{O}\left(\alpha_{s}^{2}\right)$ PQCD, must be both infrared and collinear safe. This requirement was discussed in Chapter 2.

Previously, we discussed the fact that the fragmentation process generates a transverse momentum component with respect to the jet axis (or, equivalently, an invariant mass). This 'jet-broadening' effect is not predicted by the PQCD calculations ${ }^{1}$ as it is the result of the non-perturbative hadronization process. At this point we make the assumption [34], supported by the asymptotic freedom of QCD, that the short distance behavior in which we are interested can be inferred by averaging over the remnant components of a jet. But so far we have introduced two not-so-subtle procedures to be carried out: an averaging (or combining of particles) over a jet's contents and a partitioning of an event into jets.

\section{Collinear and Infrared Safety and Jets}

One of the first theoretically sound approaches to defining jets was given by Sterman and Weinberg [38] in 1977. In their scheme, originally applied to the 3 to 2 jet transition only, the jet cross sections are written as functions of energy and angular resolution cut-offs ( $\epsilon$ and $\delta$ respectively). Thus a parton falling within a $\delta$-cone around another parton is defined to be indistinguishable from the first one within the cone. The same applies to $\epsilon$.

Another approach, the historically preferred one in $e^{+} e^{-}$annihilations [40], is based on an invariant mass cut-off. In this method, the cross sections are written in terms of the invariant masses of the various parton-parton combinations. Two partons $i$ and $j$ are then said to be indistinguishable if their invariant mass,

$$
M_{i j}^{2}=\left(P^{i}+P^{j}\right)_{\mu}\left(P^{i}+P^{j}\right)^{\mu}
$$

falls below a predetermined and arbitrary normalized threshold,

$$
y_{i j} \equiv M_{i j}^{2} / E_{c m}^{2}<M_{c u t}^{2} / E_{c m}^{2} \equiv y_{c u t} .
$$

This criterion of indistinguishability for collinear or soft partons is essential for the KLN theorem to be applicable (See Chapter 2) so that the infrared divergencies in the cross sections cancel order-by-order. We have satisfied the criterion when we define jets in such a way that the cross sections are insensitive to parton splittings - hence they are both collinear and infrared safe.

\footnotetext{
${ }^{1}$ Except that due to the soft gluons.
} 
In practice, Equation 6.1 is generalized in order to deal with massless partons. This generalization can be done in various ways to be discussed in the next section.

\section{The Algorithms for $e^{+} e^{-}$}

One of the reasons the invariant mass approach is preferred experimentally is its natural invariance (thus suitable for application at different $E_{c m}$ ) and the fact that it has only one arbitrary parameter. For these reasons we will use this jet-finding method in the present analysis.

Our ultimate objective in jet-finding is to compare the measured jet rates with the $\mathcal{O}\left(\alpha_{s}^{2}\right)$ jet rate cross sections in order to extract a value for $\Lambda_{\overline{M S}}$. The fact that these calculations deal only with up to four massless partons not only limits the accuracy of the measurement but it also introduces a recombination-scheme ambiguity. This ambiguity arises because two massless partons that fall below the threshold of Equation 6.2 acquire a mass when recombined by virtue of Equation 6.1. This extra mass must be accounted for.

The algorithms that we will use are all based on the original JADE [51] recipe and differ only in their jet measures and in their recombination schemes. An event is classified as being ' $\mathrm{n}$-jet' by the following recursive procedure:

1. An invariant mass-like jet measure $y_{i j}$ (depending on the algorithm) is calculated for every distinct pair of particles (in our case clusters) in an event.

2. Both members of a pair that falls below the resolution cut $y_{i j}<y_{\text {cut }}$ are combined into clusters using an algorithm-specific recombination scheme.

3. The process is repeated until no more clusters fall below the cut. The number of surviving clusters is then defined to be the number of jets at $y=y_{\text {cut }}$.

It is important to emphasize that the definition of a jet is in general meaningless without an accompanying resolution cut-off. The reason is simply that the range of jet multiplicity for the same event can go from 2 (high $y_{\text {cut }}$ limit) to the total number of particles in the event (low $y_{\text {cut }}$ limit). It is then only meaningful to speak of 'jetrate as a function of $y$ ' or similarly 'an $n$-jet event at $y=y_{\text {cut }}$ '. We have already encountered the resolution cut $y=y_{c u t}$ in Chapter 6 in the context of the $\mathcal{O}\left(\alpha_{s}^{2}\right)$ jet-rate calculations.

The question of how good each algorithm is will be discussed in Section 6.2.2. What follows is a description of each of the four methods used in this analysis.

\section{- JADE Algorithm}

This algorithm has been the most widely used since the inception of invariant mass based jet definitions. It uses the jet measure:

$$
y_{i j}=\frac{2 E_{i} E_{j}\left(1-\cos \theta_{i j}\right)}{E_{v i s}^{2}},
$$



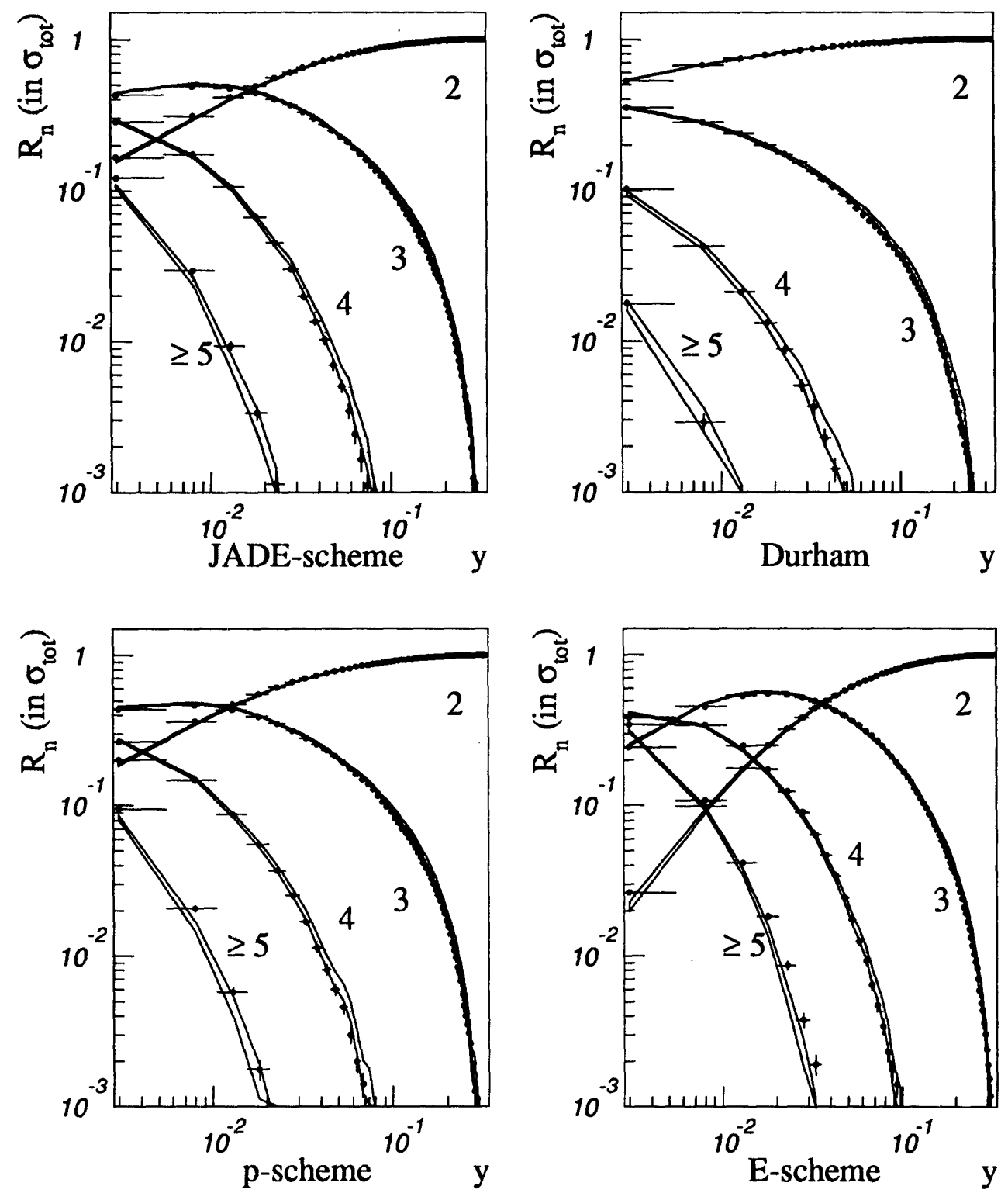

Figure 6-5: Raw jet cross section for the four algorithms considered. The dots are the Monte Carlo (Lund 6.3 PS) results with detector, triggering, and selection simulation. The two lines for each jet multiplicity correspond to 1- $\sigma$ contours on the data's statistical uncertainty. 
where $E_{v i s}$ is the sum of all the cluster energies in an event, the $E_{i}$ are the individual particle energies and $\cos \theta_{i j}$ is the angle between particles $i$ and $j$. Particles $i$ and $j$ are then recombined into particle $k$ by adding their 4-momenta:

$$
P_{k}=P_{i}+P_{j}
$$

This scheme conserves both energy and momentum but ignores particle masses in $y_{i j}$.

\section{- P-scheme}

This variant of the JADE scheme [34] uses the jet measure,

$$
y_{i j}=\frac{\left(P_{i}+P_{j}\right)^{2}}{E_{v i s}^{2}},
$$

and the recombination recipe,

$$
\begin{aligned}
\vec{p}_{k} & =\vec{p}_{i}+\vec{p}_{j} \\
E_{k} & =\left|\vec{p}_{k}\right| .
\end{aligned}
$$

This scheme conserves momentum but violates energy conservation in order to keep pseudo-particles massless.

\section{- E-scheme}

The E-scheme [34] is fully Lorentz invariant. It uses the measure,

$$
y_{i j}=\frac{\left(P_{i}+P_{j}\right)^{2}}{E_{v i s}^{2}},
$$

and recombines particles according to:

$$
P_{k}=P_{i}+P_{j}
$$

Both energy and momentum are conserved in the E-scheme. However, since recombined particles acquire mass, the comparison with the massless calculations is intrinsically inconsistent.

\section{- Durham Algorithm}

The Durham algorithm [96] is the newest of the four presented here. It uses a transverse momentum-based measure,

$$
y_{i j}=\frac{2 \min \left(E_{i}^{2}, E_{j}^{2}\right)\left(1-\cos \theta_{i j}\right)}{E_{v i s}^{2}},
$$

and the recombination recipe,

$$
P_{k}=P_{i}+P_{j} .
$$


This algorithm is especially interesting for the low- $y$ region, where the large distance behavior starts to dominate. Specifically, the problem of back-to-back soft gluon correlations [41] in the JADE algorithm is addressed.

The measured raw jet rates determined with the four algorithms just described are shown in Figure 6-5. The rates are shown as a function of the resolution parameter $y=y_{\text {cut }}$ and are defined to be (see Chapter 6) the jet multiplicities normalized to the total number of events.

The reason we have freedom in choosing a recombination scheme is that the present $\mathcal{O}\left(\alpha_{s}^{2}\right)$ QCD calculations do not handle mass. In addition, and as already mentioned, the hadronization process introduces another source of transverse momentum. We will then take the approach of assigning a systematic error on the absolute $\Lambda_{\overline{M S}}$ measurement due to the recombination scheme ambiguity.

\section{The $D_{2}(y)$ Distribution}

The jet rates defined in the previous section are per se integral distributions. As such, the contents in each bin are highly correlated with the contents of every other bin in the distribution. When fitting this distribution to a theoretical prediction, the matrix of errors becomes very complicated due to all the inter-bin correlations. For this reason, when dealing with jet rates, it is desirable to deal instead with the differential jet rate distribution $D_{2}$. The $D_{2}$ distribution was originally introduced by the OPAL experiment [97] and is defined as:

$$
D_{2}(y) \equiv \frac{R_{2}(y+\Delta y)-R_{2}(y)}{\Delta y},
$$

where $y$ is the jet resolution parameter for a particular algorithm. In this distribution each event contributes only once; this fact makes the statistical error calculation very simple since the statistical errors are then just histogram errors $\left(\approx \sqrt{N_{i}}\right)$.

\subsubsection{A Question of Resolution and Bias}

In order to ensure the stability of our unfolding procedure, we must minimize any binto-bin migrations in the histograms to be corrected. In order to do so, we must first understand the intrinsic resolution of the $D_{2}$ distribution, i.e., the correspondence of the detector level jet-rate to the parton level jet-rate. After the resolutions have been established, then the histogram bin-widths are chosen [98] to reflect these resolutions. This procedure obviously does not eliminate the bin-to-bin event migrations but it does regularize the relative rate of migration between adjacent bins. This in turn, minimizes any biases that might be introduced by the unfolding and correction procedure due to uneven 'bin-jumps'. One can summarize by saying that the correction procedure should only correct for the efficiencies of populating the various regions of 
phase-space of the variable in question; it should not deform that phase space. Doing so would introduce model-dependencies.

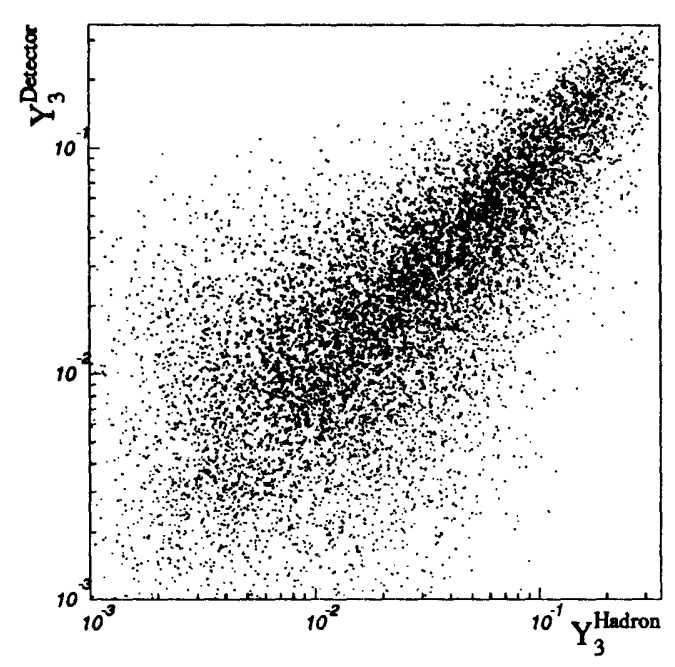

Figure 6-6: The point in $y$ at which an event turns from being 3 -jet to being 2jet $\left(y_{3}\right.$ defined in text) at the detector and hadron level.

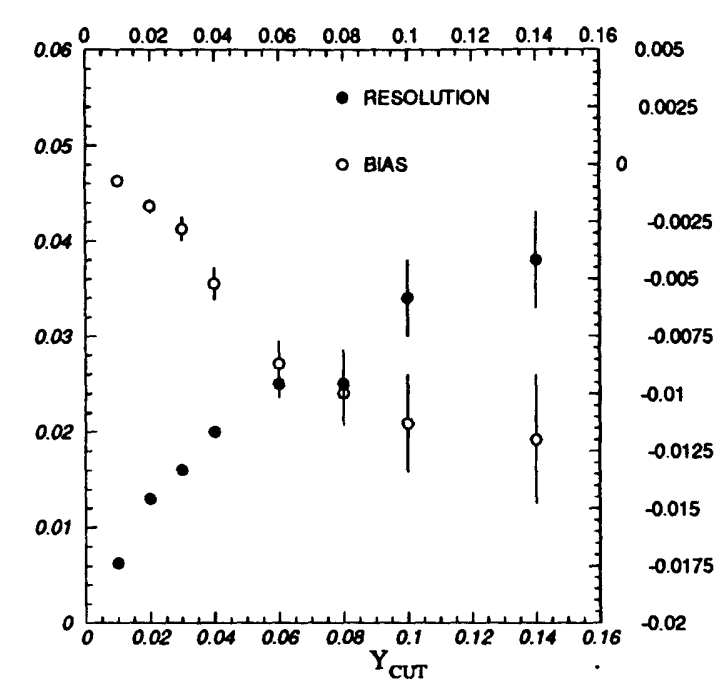

Figure 6-7: Plot of the detector-hadron resolution (full circles, vertical axis on the left) for the JADE algorithm. The bias (open circles, right vertical axis) is also included.

A detector-induced distortion also accompanies any measurement. These offsets are in general less harmful than the resolution effects. They can be treated as acceptance effects or efficiencies that can be easily corrected. We will deal with these corrections in detail in the next section. In the meantime, we can study the resolution and bias of the $D_{2}$ distribution by introducing a resolution [41] function $\Gamma$,

$$
\Gamma_{i}^{\text {det }} \equiv \frac{y_{3, i}^{\text {det }}-y_{3, i}^{h a d}}{y_{3, i}^{\text {det }}+y_{3, i}^{h a d}}
$$

in terms of $y_{3}$. The variable $y_{3}$ is defined to be the point in $y$ where an event classified as 3 -jet is re-classified as a 2 -jet event. This definition can be generalized to $y_{4}$, the point in $y$ where a 4 -jet event is re-classified as a 3 -jet event ${ }^{2} . D_{2}$ is the distribution of $y_{3}$. The subscript $i$ denotes the particular jet algorithm. Even though the underlying physics is the same, and since each algorithm has a different treatment of the invariant mass, the resolution/bias for each jet definition is in general different.

In Equation 6.4 we defined the relative resolution for the hadron to detector transformation - we can equivalently define a resolution for the parton to hadron

\footnotetext{
${ }^{2}$ More details on these definitions can be found in Appendix A.
} 

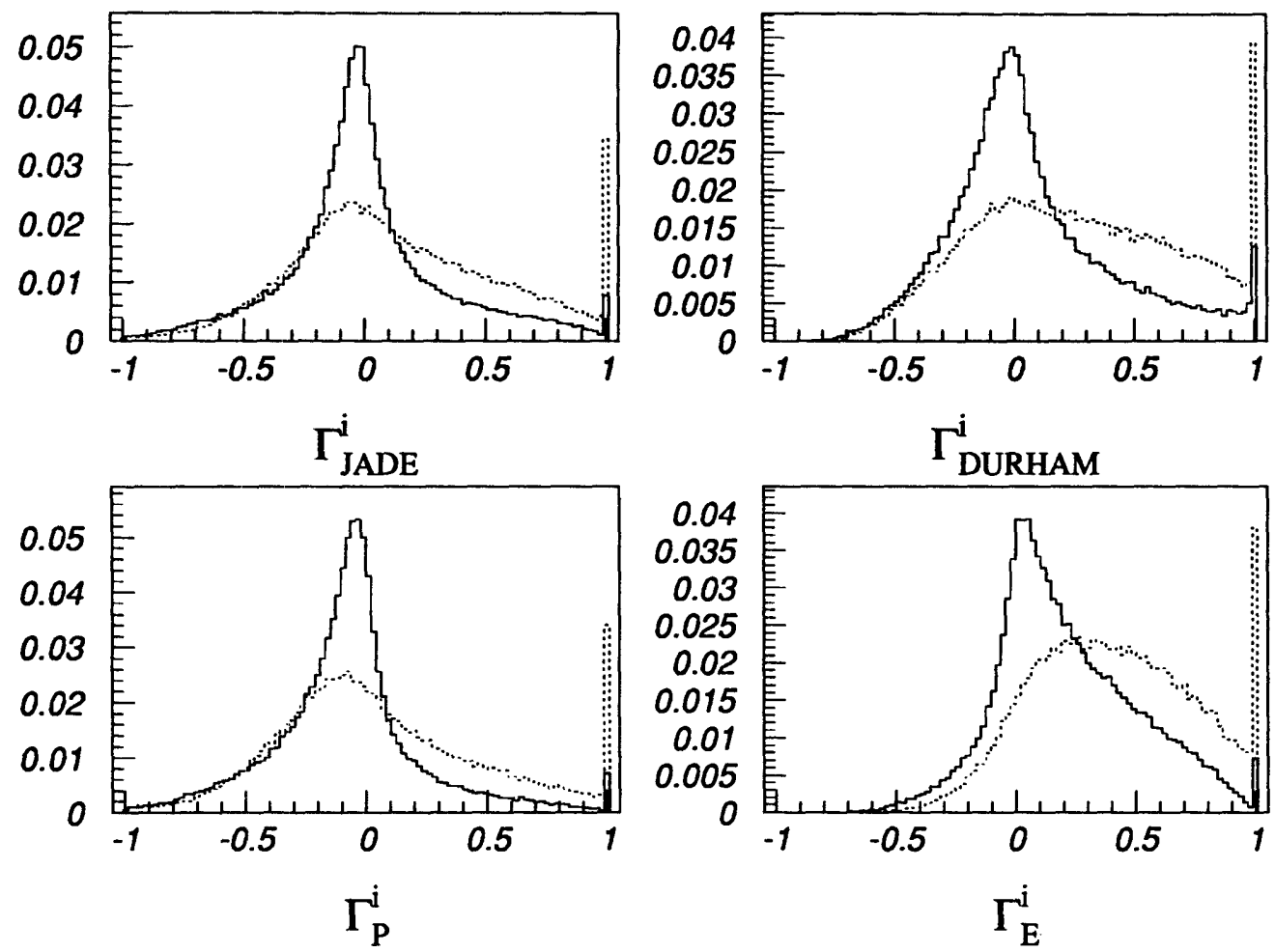

Figure 6-8: Parton-hadron resolution for $y_{3}$ (solid line) and $y_{4}$ (dashed line) for various algorithms. The vertical scale is arbitrary.

process:

$$
\Gamma_{i}^{h a d} \equiv \frac{y_{3, i}^{\text {had }}-y_{3, i}^{\text {part }}}{y_{3, i}^{\text {had }}+y_{3, i}^{\text {part }}} .
$$

Figure 6-6 shows the correlation between $y_{3}^{\text {had }}$ and $y_{3}^{\text {det }}$ for the JADE algorithm (defined previously). An offset from the diagonal to this plot reflects a bias in the algorithm response while the RMS spread from the diagonal determines the resolution. The bias and resolution are then shown in Figure 6-7 as a function of $y_{\text {cut }}$. From the figure it is evident that the relative resolution $\delta y_{3} / y_{3}$ improves with increasing $y_{\text {cut }}$. This comes as no surprise since a high- $y_{3}$ event tends to be very spread-out (non 2 -jet like) and thus more information can be extracted from the invariant mass. The small bias for the JADE algorithm is also shown in Figure 6-7.

We will apply corrections to the measured quantities in order to fix the bias problem in the next section. Figure 6-7 (and corresponding ones to the different algorithms) has been used to determine the bin widths used in the $D_{2}$ distribution measurement presented later. The chosen bin widths for the four different algorithms are included in the first row of Table 6.5, where the final measurements of $D_{2}$ are summarized. The detector resolutions were similar enough for three of the four algorithms 


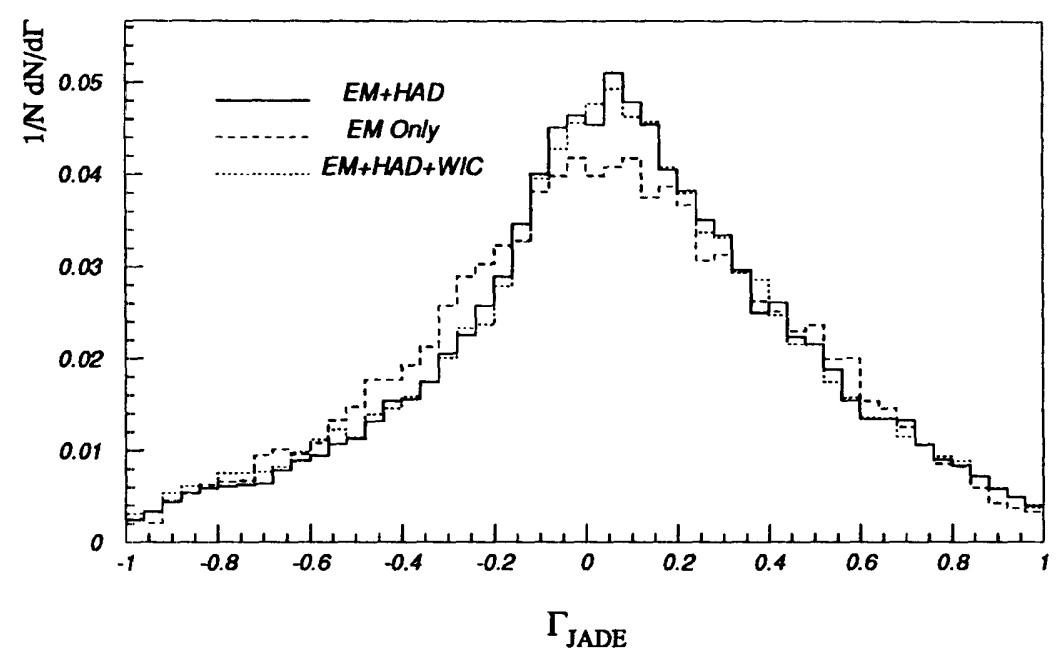

Figure 6-9: Jet resolution parameter $\Gamma_{J A D E}$ for different calorimeter layers obtained from Monte Carlo. The combination of the electromagnetic and hadronic calorimeter gives the best resolution. See the text for more details.

that identical bin widths were chosen; two sets of bin-widths are then used.

In addition, as a purely experimental exercise, we can investigate the effects (resolution-wise) of the different calorimeter subsystems on $y_{3}$. Using the Monte Carlo simulation, we can turn on and off each calorimeter subsystem at will and study the $\Gamma_{i}^{\text {det }}$ distribution. In principle, the more energy (i.e., more information) we measure, the better the resolution should be. Figure 6-9 is a plot of $\Gamma_{i}^{\text {det }}$ for a sample of JADE-reconstructed Monte Carlo events. The sample was reconstructed three times; each time one more longitudinal segmentation of the calorimeter was included in the reconstruction of jets. As Figure 6-9 shows, the optimum (by about $\approx 5 \%$ ) detector resolution is obtained when using the electromagnetic and hadronic sections of the SLD calorimeter only. This should come as no surprise as we have already determined that the dominant energy fluctuations in the WIC are due to the beam-related backgrounds (see Chapter 4); this type of fluctuation actually degrades a global quantity like $y_{3}$. Lacking a reliable method to clean-up beam backgrounds in the WIC, we will ignore the energy deposited in this third layer of the calorimeter for the rest of this analysis ${ }^{3}$.

Figure 6-8 presents the $\Gamma_{i}^{\text {had }}$ distributions for the four algorithms considered here. The analogous distribution for $y_{4}$ is also shown. The offset from zero corresponds to the overall bias and the width of the distribution is the overall resolution. It is clear from the figure that the JADE scheme suffers the least from the hadronization effects. The E-scheme is by far the worst.

\footnotetext{
${ }^{3}$ It is safe to ignore the energy deposition in the WIC since over $90 \%$ of the event energy (see Table 4.3) is absorbed in the first two layers of the calorimeter (the LAC).
} 


\subsection{Corrections to the Jet-Fractions}

We have already established what we mean by a jet, both experimentally and theoretically. We have seen that the algorithms based on invariant-mass resolution criteria lend themselves to analytic calculations. In addition, in Chapter 2 we also established that these calculations are limited to $\mathcal{O}\left(\alpha_{s}^{2}\right)$ in perturbation theory and thus describe at most 4 partons and do not describe the hadronization process at all. It is clear then, that in order to compare theory with experiment, we must somehow propagate our theoretical expectations (parton level) to our experimental arena (detector level), or vice versa - the point being that, in order to compare effectively the partons producing the jets with the detected jets, there must be a complete accounting of all of the distortions created between the two ends.

\section{The Smearing of the Partons}

In order to classify all of the possible distortions, it is useful to outline the process that gives us hadronic events in the calorimeter. We can divide this process into five stages:

$$
\begin{gathered}
\left(\mathbf{I}: e^{ \pm} \rightarrow e^{ \pm} \gamma\right) \sim\left(\mathbf{I I}: e^{+} e^{-} \rightarrow q \bar{q}\right) \sim(\text { III: } q \bar{q} \rightarrow \text { hadrons }) \sim \\
(\mathbf{I V}: \text { hadrons } \rightarrow \text { hadrons }+ \text { decayed particles }) \sim \\
(\mathbf{V}: \text { hadrons }+ \text { decayed particles } \rightarrow \text { detector })
\end{gathered}
$$

The Roman numerals label each stage and the squiggly arrows represent the sequential ordering. In the next subsections we will describe each of the above stages in more detail and will discuss their relevance in comparing with theory.

Stage $I$, initial state radiation, is well understood, calculable, and effectively just an asymmetric smearing of the center-of-mass energy. Stage $I I$ above corresponds to the PQCD $e^{+} e^{-}$jet calculations. There is no ambiguity (except for the recombination scheme) at this stage; four well defined partons and their relative production rates are given by analytic formulae. Stages $I V$ and $V$ are also well defined; given a particle that impinges on the detector we ought to be able to know the probability that it will decay and what the detector will see.

Stage $I I I$, the hadronization process, is not yet calculable in QCD. We discussed this aspect in Chapter 2. Here, in order to be able to make the measurement, we will have to rely on hadronization models. We will use the JETSET and HERWIG models discussed in Chapter 2 as black boxes that link stage $I$ with stage $V$ above. The price we pay in using such a black box approach is in the introduction of a systematic error due to hadronization - a statement of our ignorance about such a process. More on that later. 


\section{Correction Procedure}

In the above diagram (recalling the terminology from the previous two sections), the smearing and distortions increase from left to right. This implies that, in order to translate the data to parton level, we must unfold it for stages I-IV. Equivalently, in order to translate the calculations to detector level, we must fold them for stages $I$, $I I I, I V$ and $V$. At this point we choose our correction procedure on two principles: that it allows us to compare our measurement with other measurements and that it minimizes any model dependencies. We thus choose to perform our measurement at the hadron level by folding the PQCD calculations for fragmentation effects and by unfolding the raw measurement for detector effects.

We define a corrected $D_{2}$ distribution as that derived from the corrected jetrates. During each stage of the correction, the jet rates are renormalized to satisfy $\sum_{n} R_{n}=1$. We will now describe each of these procedures in more detail. Appendix B covers the details of Section 6.3.4.

\subsubsection{Initial State Radiation}

Initial state radiation (ISR) is the emission of photons by the initial $e^{+} e^{-}$. It has the effect of shifting the effective center-of-mass energy of an event. QCD calculations generally do not include this effect; it is usually included in the Electroweak-QED effects that are simulated, together with the decay of the $q \bar{q}$ pair, by the Monte Carlo generators. In order to compare any such calculation with the data we must then correct the data for this effect. The net result of ISR is a small distortion on the 3-jet rate of $\approx 1-3 \%$. A small ISR correction is thus applied to the measured jet-rates. Since ISR is a pure QED effect independent of any QCD model, we correct the measured distributions using the factor method. Following the notation of Appendix B, we write:

$$
C_{n}^{\gamma}(y)=\frac{R_{n}^{N o I S R}(y)}{R_{n}^{I S R}(y)},
$$

where $n$ is the jet index and the $R_{n}$ are the jet rates obtained with two parton level Monte Carlo samples: one with ISR and one without ISR. Each of the four algorithms has a different set of correction factors.

\subsubsection{Hadronization Effects}

Since we have no way of knowing on a particle by particle basis which hadrons come from which jet (or in which order), the fragmentation process smears the measurement of a parton's direction. This distortion is evident in Figure 6-11, where both the hadron and the parton-level jet rates are shown for the JETSET PS Monte Carlo with string fragmentation. Since the fragmentation process is inherently low $-Q^{2}$, this distortion has more of an effect at low invariant masses (low $y$ ). 

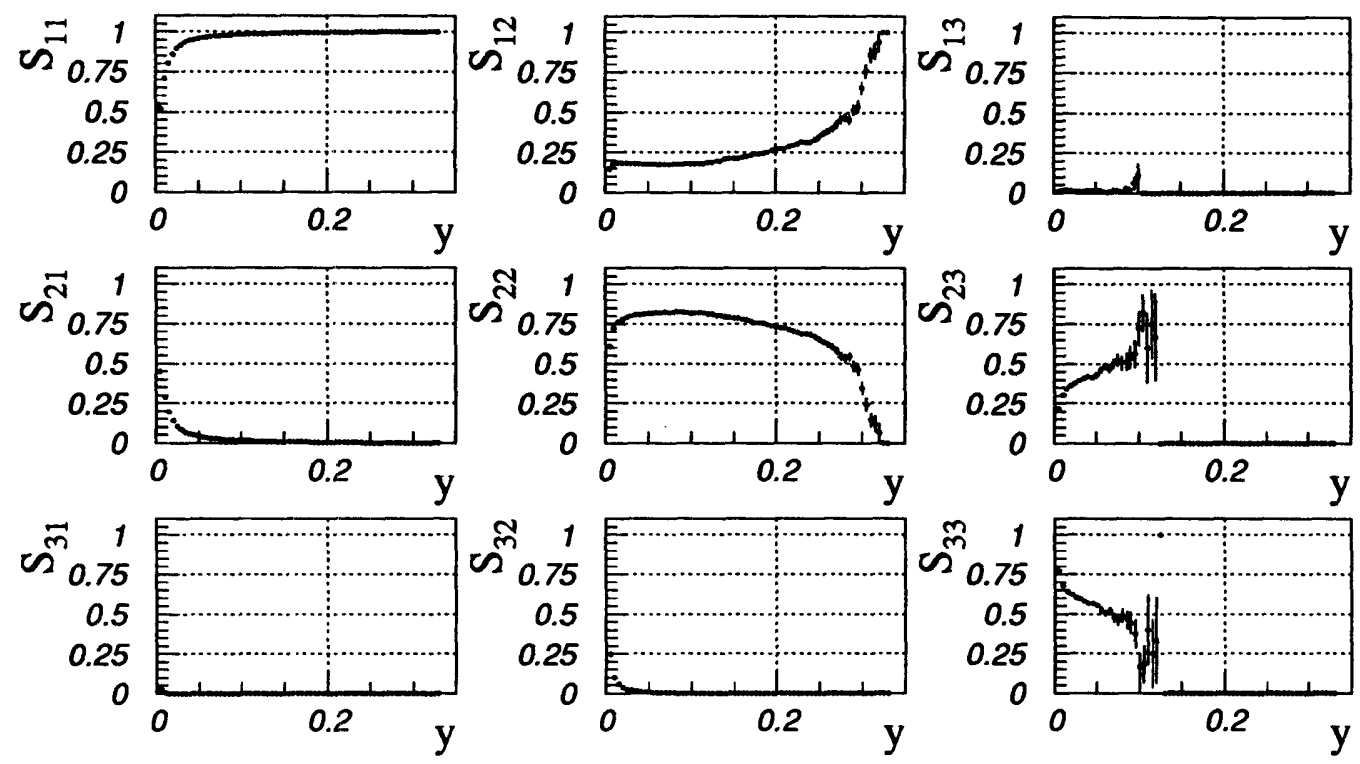

Figure 6-10: The hadronization smearing matrix $S(y)$ for the JETSET 6.3 PS model, JADE scheme; the definition of $S_{i j}$ is given in Equation 6.9. The error bars are statistical only.

The hadronization effects are parameterized by a smearing matrix that distorts the parton level jet rates. In our case this distortion will be applied to the $\mathcal{O}\left(\alpha_{s}^{2}\right)$ calculation. This smearing is different for each of the fragmentation models, being dependent on the jet resolution parameter $y$, and is different for each of the jet algorithms. Following the notation of Appendix B, we write:

$$
\vec{P}(y) \equiv\left(\begin{array}{c}
R_{2}^{p}(y) \\
R_{3}^{p}(y) \\
R_{\geq 4}^{p}(y)
\end{array}\right) \quad \vec{H}(y) \equiv\left(\begin{array}{c}
R_{2}^{h}(y) \\
R_{3}^{h}(y) \\
R_{\geq 4}^{h}(y)
\end{array}\right)
$$

where $R_{2}$ denotes the 2 jet rate, $R_{3}$ the 3 jet rate, and $R_{\geq_{4}}$ the four and higher jet rate. The superscripts $p$ and $h$ label the jet rates as being calculated at either the parton or at the hadron level. The folding procedure consists in finding the matrix $S(y)$

$$
\vec{H}(y)=S(y) \vec{P}(y),
$$

that parameterizes, in $3 \times 3$ form, the $y$-dependence of the hadronization effects. There is a different $S(y)$ for each model. In practice each matrix was obtained by generating 100,000 Monte Carlo events with no initial state radiation and tabulating the jet multiplicity correlations between the parton and the hadron level. This matrix 

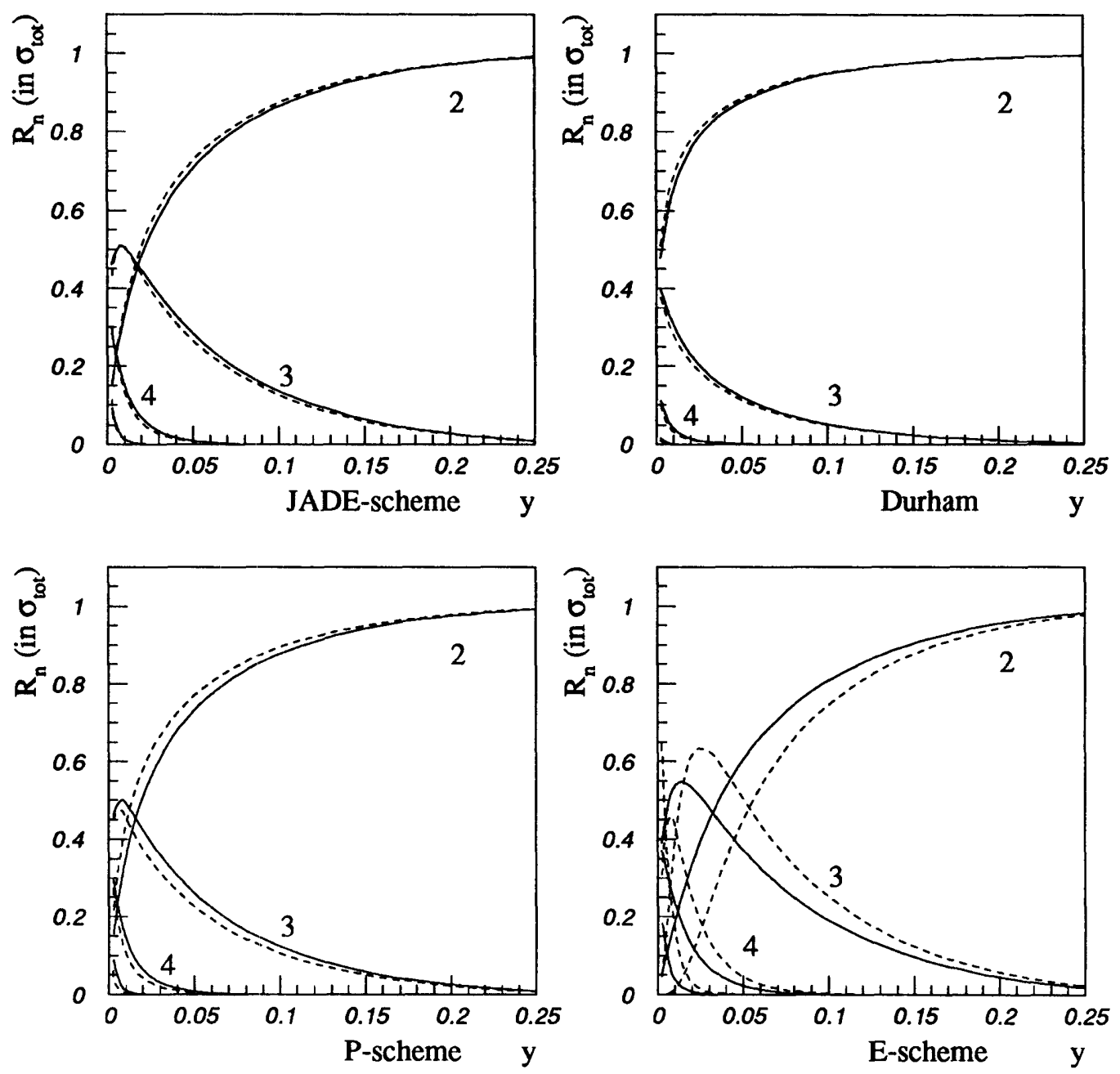

Figure 6-11: Parton (line) and hadron (dashed) level jet cross section for the four algorithms considered as a function of the $y$; shown are results for the JETSET Monte Carlo. The $R_{\geq 5}$ jet rate is the unlabelled pair of curves at the bottom left corner of each plot.

of transition probabilities is defined as,

$$
S_{i j}=\frac{\text { number of } i \text {-jet events (parton) that look like } j \text {-jet (hadron) }}{\text { total number of } i \text {-jet events (parton) }} .
$$

An example of a matrix $S(y)$ is presented in Figure 6-10. Using different models, i.e. different matrices, to fold the parton distributions will give us an estimate of the systematic uncertainties due to hadronization and modelling parameters. Figure 6-11 shows the jet rates at the parton and hadron levels. We can see from the figure that 
both the JADE and Durham algorithms do a good job in correlating the parton and hadron level jet rates. This good correlation translates into relatively small correction factors and reduced systematic uncertainties (to be discussed later).

It is also evident from the figure that the E-scheme algorithm has very large correction factors. For this reason we will not include the results obtained by this algorithm in the final measurement although its analysis will be carried through to the end.

\subsubsection{Particle Decays}

The correction for particle decays (stage $I V$ ) is implicitly included in the correction for hadronization effects. In the latter, particles with a lifetime of $\tau \geq 10^{-10}$ seconds are treated as stable particles. They are then allowed to decay at the detector level only. This is to ensure that we do not have to include any magnetic field bending effects in the pure hadronization corrections (these effects are taken care of by the detector simulation). It also guarantees a consistent definition of a hadron (in terms of decays) in the hadronization and detector correction stages.

\subsubsection{Detector Effects}

Any measurement performed with a particle detector will inevitably be distorted due to the finite resolution and acceptance of the device. If one understands the detector apparatus, and provided the detector is linear enough, then this distortion can be corrected by using simple methods that effectively divide-out these distortions. The two methods considered for this analysis, the inversion and the factor method, are described in detail in Appendix B.

The factor method is essentially the same as the ISR correction factor described in Section 6.3.1. In this case the jet rates used are calculated at the detector and hadron level; we denote the $n$-jet correction factor at $y=y_{\text {cut }}$ as $C_{n}^{d}(y)$. The inversion method takes care of the correlations between different jet multiplicities. The definition of the correction matrix is very similar to the one for the hadronization smearing matrix (see Section 6.3.2), except that this time the inverse of the matrix is used to unfold the data. We denote the inversion correction factor by $\left(T^{-1}\right)_{n m}$. Details of these procedures are presented in Appendix B.

In Appendix B we show that the factor method has an advantage over the matrix method when dealing with a limited Monte Carlo sample. We also show that the factor correction method is linear over a wide range of input parameters. The error calculations are also simpler. For these reasons we choose to use the factor method for the final measurement; however, we will carry parallel measurements with the inversion method as a cross-check on the preferred method. Figure 6-12 shows the correction factor for the factor method; Figure B-1, in Appendix B, shows the correction matrix used in the inversion method. 


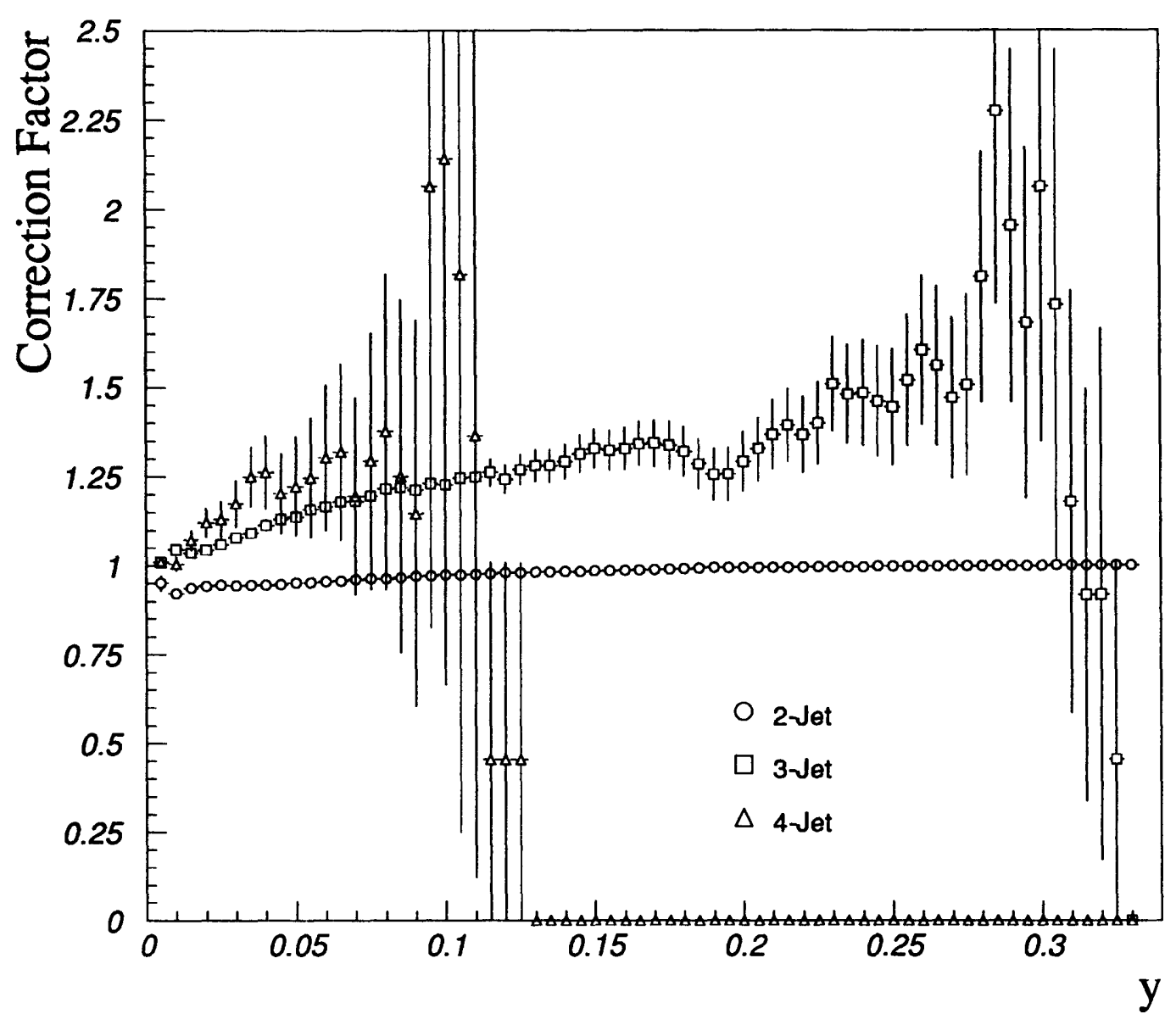

Figure 6-12: This plot presents the jet rate correction factors used in this analysis with the JADE algorithm. These factors were calculated by comparing the jet-rates at the hadron and detector levels using the recipe presented in Appendix B.

\subsubsection{Putting It All Together}

Now that we have defined the correction steps to follow, we can proceed with transforming the raw jet rates of Figure 6-5 into corrected jet rates. We do so by,

$$
R_{n}^{h}(y)= \begin{cases}C_{n}^{\gamma}(y) C_{n}^{d}(y) R_{n}^{d}(y) & \text { factor method } \\ C_{n}^{\gamma}(y) \sum_{m}\left(T^{-1}\right)_{n m} R_{m}^{d}(y) & \text { inversion method }\end{cases}
$$

where the superscript $h$ refers to hadron level and $d$ to detector level jet rates. $R_{n}^{h}(y)$ is then the hadron-level $n$-jet rate at the jet resolution point $y$. Once the raw jet rates are corrected, the measured $D_{2}$ distribution is derived using Equation 6.3. The result of the factor method corrected jet rate cross section measurement is presented in Tables 6.1 to 6.4 for the four algorithms considered. The resultant $D_{2}$ distribution 
is presented in Table 6.5 for the two correction procedures. The measurement shows that there are no significant differences between the two procedures.

\section{Uncertainties}

The errors in the jet rate cross section presented in Tables 6.1 to 6.4 are a combination of statistical and systematic uncertainties. The statistical uncertainties include the errors associated with a limited data and Monte Carlo sample size. These errors are the result of propagating the statistical uncertainties in Equation 6.10.

In Chapter 4 we discussed the effect of a limited data sample on the tuning of the detector's simulation Monte Carlo. Based on that discussion, we assign a conservative systematic uncertainty which is equal to the statistical error due to the data sample size alone. The reasoning is that the best one can do in tuning a detector is to tune it to the level of the data sample used; therefore the statistical uncertainties of the data sample itself is the best one can achieve. The statistical and systematic uncertainties are then combined in quadrature.

The systematic errors on the $D_{2}$ distribution are derived from the systematic errors on the jet rates. The bin-to-bin correlations introduced by the Monte Carlo statistical and systematic errors are taken into account. The statistical uncertainties are derived from the $D_{2}$ distribution itself by using the multinomial uncertainties of the histogram ${ }^{4}$. Once again, the statistical and systematic uncertainties are combined in quadrature.

\subsection{Fit for $\Lambda_{\overline{M S}}$}

Now that the data have been corrected for detector effects we can proceed to fit for $\Lambda_{\overline{M S}}$. We fit to the ERT calculations discussed in Chapter 2 by using the minimization program MINUIT [99] and by minimizing the $\chi^{2}$ function:

$$
\chi^{2}\left(\Lambda_{\overline{M S}}, f\right)=\sum_{j \in\{\text { bins }\}} \frac{\left(D_{2}^{\text {th }}\left(y_{j} ; \Lambda_{\overline{M S}}, f\right)-D_{2}^{\text {data }}\left(y_{j}\right)\right)^{2}}{\left(\sigma_{j}^{t h}\right)^{2}+\left(\sigma_{j}^{s y s}\right)^{2}}
$$

where $D_{2}^{\text {th }}$ is the hadronization-smeared distribution derived from the ERT $\mathcal{O}\left(\alpha_{s}^{2}\right)$ calculations (See Chapter 2),

$$
D_{2}^{t h}\left(y_{i}\right)=\frac{\sum_{i=1}^{4} S_{2 i+1} R_{2}\left(y_{i+1} ; \Lambda_{\overline{M S}}, f\right)-S_{2 i} R_{2}\left(y_{i} ; \Lambda_{\overline{M S}}, f\right)}{\Delta y_{i}}
$$

$D_{2}^{\text {data }}$ is the experimental distribution from Table 6.5 , and $f=\mu^{2} / Q^{2}$ is the renor-

\footnotetext{
${ }^{4}$ Due to the small number of bins (11) and the large differences in bin occupancies, we can no longer use the Gaussian approximation.
} 


\begin{tabular}{||c|c|r|r||}
\hline \hline \multicolumn{4}{|c||}{ JADE } \\
\hline$y_{i}$ & \multicolumn{1}{|c|}{$R_{2}^{h}$} & \multicolumn{1}{c|}{$R_{3}^{h}$} & \multicolumn{1}{c|}{$R_{\geq 4}^{h}$} \\
\hline \hline 0.005 & $15.07 \pm 0.61$ & $44.77 \pm 0.86$ & $40.16 \pm 0.84$ \\
0.015 & $37.06 \pm 0.82$ & $50.99 \pm 0.87$ & $11.96 \pm 0.57$ \\
0.030 & $56.41 \pm 0.84$ & $39.76 \pm 0.85$ & $3.84 \pm 0.35$ \\
0.045 & $67.41 \pm 0.79$ & $31.26 \pm 0.82$ & $1.34 \pm 0.21$ \\
0.065 & $77.09 \pm 0.71$ & $22.47 \pm 0.74$ & $0.43 \pm 0.13$ \\
0.080 & $81.45 \pm 0.66$ & $18.42 \pm 0.70$ & $0.14 \pm 0.07$ \\
0.090 & $84.32 \pm 0.62$ & $15.64 \pm 0.65$ & $0.04 \pm 0.04$ \\
0.115 & $88.74 \pm 0.53$ & $11.26 \pm 0.57$ & - \\
0.145 & $92.40 \pm 0.44$ & $7.60 \pm 0.48$ & - \\
0.180 & $95.91 \pm 0.33$ & $4.09 \pm 0.36$ & - \\
0.225 & $98.36 \pm 0.22$ & $1.64 \pm 0.23$ & - \\
0.275 & $99.55 \pm 0.11$ & $0.45 \pm 0.13$ & - \\
\hline \hline
\end{tabular}

Table 6.1: The measured jet cross section using the JADE algorithm in $\%$ of $\sigma_{\text {tot }}$ as a function of the jet resolution parameter $y_{i}$. The jet-fractions have been corrected for detector effects and initial state radiation as described in the text.

\begin{tabular}{||c|c|r|r||}
\hline \hline \multicolumn{4}{||c||}{ P-scheme } \\
\hline$y_{i}$ & \multicolumn{1}{|c||}{$R_{2}^{h}$} & \multicolumn{1}{c|}{$R_{3}^{h}$} & \multicolumn{1}{c|}{$R_{\geq 4}^{h}$} \\
\hline \hline 0.005 & $19.59 \pm 0.68$ & $47.09 \pm 0.87$ & $33.32 \pm 0.81$ \\
0.015 & $45.35 \pm 0.85$ & $45.40 \pm 0.86$ & $9.25 \pm 0.51$ \\
0.030 & $62.54 \pm 0.82$ & $34.64 \pm 0.83$ & $2.83 \pm 0.30$ \\
0.045 & $72.75 \pm 0.76$ & $26.21 \pm 0.77$ & $1.04 \pm 0.18$ \\
0.065 & $80.44 \pm 0.67$ & $19.22 \pm 0.70$ & $0.34 \pm 0.11$ \\
0.080 & $84.37 \pm 0.62$ & $15.52 \pm 0.64$ & $0.11 \pm 0.07$ \\
0.090 & $86.35 \pm 0.58$ & $13.61 \pm 0.62$ & $0.04 \pm 0.04$ \\
0.115 & $90.12 \pm 0.50$ & $9.88 \pm 0.54$ & - \\
0.145 & $93.77 \pm 0.41$ & $6.23 \pm 0.44$ & - \\
0.180 & $96.44 \pm 0.31$ & $3.56 \pm 0.34$ & - \\
0.225 & $98.38 \pm 0.21$ & $1.62 \pm 0.23$ & - \\
0.275 & $99.68 \pm 0.10$ & $0.32 \pm 0.10$ & - \\
\hline \hline
\end{tabular}

Table 6.2: The measured jet-fractions using the P-scheme variant of the JADE algorithm (in $\%$ of $\sigma_{t o t}$ ) as a function of the jet resolution parameter $y_{c u t}$. The jet-fractions have been corrected for detector effects and initial state radiation as described in the text. 


\begin{tabular}{||c|r|r|r||}
\hline \hline \multicolumn{4}{|c||}{ E-scheme } \\
\hline$y_{i}$ & \multicolumn{1}{|c|}{$R_{2}^{h}$} & \multicolumn{1}{c||}{$R_{3}^{h}$} & \multicolumn{1}{c|}{$R_{\geq 4}^{h}$} \\
\hline \hline 0.005 & $0.09 \pm 0.04$ & $8.55 \pm 0.44$ & $91.36 \pm 0.57$ \\
0.015 & $4.55 \pm 0.35$ & $52.53 \pm 0.85$ & $42.80 \pm 0.91$ \\
0.030 & $22.27 \pm 0.69$ & $62.65 \pm 0.87$ & $15.04 \pm 0.66$ \\
0.045 & $39.28 \pm 0.81$ & $54.46 \pm 0.90$ & $6.24 \pm 0.45$ \\
0.065 & $56.37 \pm 0.83$ & $41.19 \pm 0.88$ & $2.43 \pm 0.32$ \\
0.080 & $65.31 \pm 0.80$ & $33.60 \pm 0.85$ & $1.09 \pm 0.22$ \\
0.090 & $70.22 \pm 0.77$ & $29.31 \pm 0.82$ & $0.47 \pm 0.14$ \\
0.115 & $78.80 \pm 0.68$ & $21.05 \pm 0.74$ & $0.15 \pm 0.08$ \\
0.145 & $86.19 \pm 0.57$ & $13.81 \pm 0.63$ & - \\
0.180 & $91.30 \pm 0.47$ & $8.70 \pm 0.52$ & - \\
0.225 & $95.61 \pm 0.33$ & $4.39 \pm 0.39$ & - \\
0.275 & $98.39 \pm 0.20$ & $1.61 \pm 0.23$ & - \\
\hline \hline
\end{tabular}

Table 6.3: The measured jet cross section using the E-scheme variant of the JADE algorithm (in $\%$ of $\sigma_{\text {tot }}$ ) as a function of the jet resolution parameter $y_{\text {cut }}$. The jet-fractions have been corrected for detector effects and initial state radiation as described in the text.

\begin{tabular}{||c|c|r|r||}
\hline \hline \multicolumn{4}{|c||}{ Durham } \\
\hline$y_{i}$ & \multicolumn{1}{|c||}{$R_{2}^{h}$} & \multicolumn{1}{c||}{$R_{3}^{h}$} & \multicolumn{1}{c||}{$R_{\geq 4}^{h}$} \\
\hline \hline 0.005 & $50.41 \pm 0.85$ & $37.85 \pm 0.84$ & $11.74 \pm 0.57$ \\
0.010 & $63.82 \pm 0.82$ & $31.27 \pm 0.81$ & $4.91 \pm 0.39$ \\
0.015 & $70.81 \pm 0.77$ & $26.51 \pm 0.77$ & $2.67 \pm 0.30$ \\
0.020 & $75.46 \pm 0.73$ & $22.92 \pm 0.74$ & $1.61 \pm 0.23$ \\
0.030 & $81.50 \pm 0.66$ & $17.69 \pm 0.67$ & $0.81 \pm 0.17$ \\
0.045 & $86.41 \pm 0.58$ & $13.39 \pm 0.61$ & $0.20 \pm 0.10$ \\
0.065 & $90.37 \pm 0.50$ & $9.60 \pm 0.53$ & $0.03 \pm 0.03$ \\
0.080 & $92.64 \pm 0.44$ & $7.35 \pm 0.47$ & $0.01 \pm 0.02$ \\
0.095 & $94.31 \pm 0.39$ & $5.69 \pm 0.41$ & - \\
0.135 & $96.68 \pm 0.30$ & $3.32 \pm 0.33$ & - \\
0.185 & $98.28 \pm 0.21$ & $1.72 \pm 0.25$ & - \\
0.245 & $99.71 \pm 0.09$ & $0.29 \pm 0.10$ & - \\
\hline \hline
\end{tabular}

Table 6.4: The measured jet cross section using the Durham algorithm (in \% of $\sigma_{\text {tot }}$ ) as a function of the jet resolution parameter $y_{c u t}$. The jet-fractions have been corrected for detector effects and initial state radiation as described in the text. 


\begin{tabular}{||c||c|r|r|r||}
\hline \hline \multicolumn{5}{|c||}{ Measured $D_{2}\left(y_{i}\right)$} \\
\hline$y_{i}-y_{i+1}$ & \multicolumn{1}{|c|}{ JADE } & \multicolumn{1}{c|}{ E-scheme } & \multicolumn{1}{c|}{ p-scheme } & \multicolumn{1}{c|}{ (Durham) } \\
\hline \hline $0.005-0.015$ & $22.00 \pm 0.81$ & $4.45 \pm 0.45$ & $25.76 \pm 0.80$ & $26.83 \pm 1.09$ \\
$(0.005-0.010)$ & $21.96 \pm 1.45$ & $2.87 \pm 1.95$ & $26.56 \pm 1.35$ & $25.74 \pm 1.31$ \\
\hline $0.015-0.030$ & $12.89 \pm 0.43$ & $11.81 \pm 0.41$ & $11.46 \pm 0.39$ & $13.99 \pm 0.90$ \\
$(0.010-0.015)$ & $13.50 \pm 0.78$ & $11.74 \pm 0.90$ & $11.77 \pm 0.66$ & $13.40 \pm 1.03$ \\
\hline $0.030-0.045$ & $7.33 \pm 0.33$ & $11.34 \pm 0.43$ & $6.81 \pm 0.29$ & $9.30 \pm 0.78$ \\
$(0.015-0.020)$ & $7.11 \pm 0.63$ & $11.52 \pm 0.95$ & $7.00 \pm 0.54$ & $9.76 \pm 0.92$ \\
\hline $0.045-0.065$ & $4.84 \pm 0.21$ & $8.54 \pm 0.32$ & $3.84 \pm 0.20$ & $6.03 \pm 0.41$ \\
$(0.020-0.030)$ & $5.04 \pm 0.43$ & $9.01 \pm 0.75$ & $3.92 \pm 0.36$ & $6.42 \pm 0.48$ \\
\hline $0.065-0.090$ & $2.89 \pm 0.15$ & $5.54 \pm 0.24$ & $2.36 \pm 0.15$ & $3.28 \pm 0.25$ \\
$(0.030-0.045)$ & $2.86 \pm 0.31$ & $5.03 \pm 0.59$ & $2.38 \pm 0.26$ & $3.37 \pm 0.30$ \\
\hline $0.090-0.115$ & $1.77 \pm 0.13$ & $3.43 \pm 0.20$ & $1.51 \pm 0.13$ & $1.98 \pm 0.17$ \\
$(0.045-0.065)$ & $1.85 \pm 0.29$ & $3.44 \pm 0.56$ & $1.56 \pm 0.23$ & $1.80 \pm 0.20$ \\
\hline $0.115-0.145$ & $1.22 \pm 0.10$ & $2.46 \pm 0.15$ & $1.22 \pm 0.11$ & $1.31 \pm 0.11$ \\
$(0.065-0.095)$ & $1.23 \pm 0.22$ & $2.47 \pm 0.45$ & $1.30 \pm 0.18$ & $1.47 \pm 0.14$ \\
\hline $0.145-0.180$ & $1.00 \pm 0.09$ & $1.46 \pm 0.11$ & $0.76 \pm 0.08$ & $0.59 \pm 0.07$ \\
$(0.095-0.135)$ & $1.11 \pm 0.18$ & $1.46 \pm 0.35$ & $0.81 \pm 0.15$ & $0.53 \pm 0.09$ \\
\hline $0.180-0.225$ & $0.54 \pm 0.06$ & $0.96 \pm 0.07$ & $0.43 \pm 0.06$ & $0.32 \pm 0.05$ \\
$(0.135-0.185)$ & $0.60 \pm 0.13$ & $0.99 \pm 0.26$ & $0.44 \pm 0.11$ & $0.35 \pm 0.07$ \\
\hline $0.225-0.275$ & $0.24 \pm 0.05$ & $0.56 \pm 0.05$ & $0.26 \pm 0.05$ & $0.24 \pm 0.04$ \\
$(0.185-0.250)$ & $0.22 \pm 0.11$ & $0.61 \pm 0.22$ & $0.38 \pm 0.09$ & $0.26 \pm 0.06$ \\
\hline $0.275-0.330$ & $0.08 \pm 0.04$ & $0.28 \pm 0.03$ & $0.06 \pm 0.03$ & $0.03 \pm 0.02$ \\
$(0.250-0.330)$ & $0.12 \pm 0.09$ & $0.51 \pm 0.19$ & $0.07 \pm 0.08$ & $0.05 \pm 0.03$ \\
\hline \hline
\end{tabular}

Table 6.5: The measured $D_{2}$ distribution corrected for detector effects and initial state radiation. For each bin, two entries are given. The top row corresponds to the data corrected by the factor method (as derived from Tables 6.1, 6.2, 6.3, and 6.4). The bottom row has been corrected by the matrix inversion method. Note the difference in binning for the Durham algorithm (denoted by parentheses). 


\begin{tabular}{||c|r|r|c|r||}
\hline \hline Scheme & \multicolumn{1}{|c|}{$\Lambda_{\overline{M S}}^{(5)}$} & $\mu^{2} / Q^{2}$ & fit range & $\chi^{2} / N d F$ \\
\hline \multirow{2}{*}{ JADE } & $363_{-66}^{+76}$ & 1.0 (fixed scale) & $0.045-0.25(35)$ & $7.1 / 6$ \\
& $151_{-19}^{+21}$ & $(48 \pm 35) \times 10^{-4}$ & $0.015-0.25(62)$ & $8.5 / 7$ \\
\hline \multirow{2}{*}{ Durham } & $436_{-87}^{+99}$ & 1.0 (fixed scale) & $0.030-0.20(18)$ & $4.8 / 6$ \\
& $236_{-28}^{+32}$ & $\left(47_{-47}\right) \times 10^{-3}$ & $0.005-0.20(51)$ & $7.3 / 8$ \\
\hline \multirow{2}{*}{$\mathrm{P}$} & $357 \pm 69$ & 1.0 (fixed scale) & $0.045-0.25(27)$ & $9.9 / 6$ \\
& $250_{-30}^{+34}$ & $(64 \pm-173) \times 10^{-3}$ & $0.015-0.25(55)$ & $11.8 / 7$ \\
\hline \multirow{2}{*}{$\mathrm{E}$} & $572_{-131}^{+154}$ & 1.0 (fixed scale) & $0.090-0.25(29)$ & $6.6 / 4$ \\
& $165_{-28}^{+26}$ & $(48 \pm 4.5) \times 10^{-6}$ & $0.045-0.25(60)$ & $2.3 / 5$ \\
\hline \hline
\end{tabular}

Table 6.6: Results of the fits to $\Lambda_{\overline{M S}}$. Units are in $\mathrm{MeV}$. The numbers in parenthesis in the 'fit range' column are the fraction, in \%, of the 9,878 total events contained in the fit range. For each algorithm the upper row corresponds to the fit with $f=1.0$ and the lower row corresponds to the fit with $f$ as a free parameter. The errors quoted are those returned by MINUIT on the $\chi^{2}$ defined in Equation 6.11 .

malization scale. The $R_{2}$ in Equation 6.11 is taken from Equation 2.23; the matrix $S_{i j}$ is the fragmentation smearing matrix defined in Equation 6.9. The denominator of 6.11 contains the statistical error derived from the theoretical distribution and the systematic error derived from the experimental distribution. Thus, $\sigma_{j}^{s y s}$ is the systematic uncertainty for bin $j$ from Table 6.5. The theoretical uncertainty, $\sigma_{j}^{\text {th }}$, is just the statistical error calculated from the theoretical expectation for a data sample the same size as our experimental sample $(9,878 \text { events })^{5}$.

The fit range for the $D_{2}$ distribution was determined using both experimental and theoretical criteria. First of all, to avoid problems in the corrected distributions, we imposed the requirement that the selected events fall in a region of phase space where the detector correction factors (for $R_{2}$ and $R_{3}$ ) were within $\pm 30 \%$ of 1.0 . In addition, we excluded fit regions with large fluctuations in the correction factors. This requirement set the upper fit limit for the four algorithms partly because of the reduced number of multi-jet events at high $y$.

We now proceed with the fit. The rest of the discussion centers on the results of Table 6.6 which are graphically presented in Figure 6-13.

The most important issue when performing this fit is the choosing of a proper renormalization scale. We know from Chapter 2 that the scale dependence of the NLO terms in Equation 2.23 is just a relic of the finite truncation of the perturbative series (e.g., Equation 2.5). In addition, the renormalization group equation tells us that

\footnotetext{
${ }^{5}$ In practice, if we had used the statistical errors from the data, the effect on our final measurement of $\alpha_{s}$ would be $\Delta \alpha_{s}=+0.0006$. This approach, although amply used in the literature, generally introduces a bias since the derivation of the $\chi^{2}$ distribution uses the uncertainties in the candidate distribution.
} 
any $\mathcal{O}\left(\alpha_{s}^{2}\right)$ perturbatively calculated observable is insensitive to the renormalization subtraction scale up to $\mathcal{O}\left(\alpha_{s}^{2}\right)$. This implies that any residual dependence of $\alpha_{s}$ on the renormalization scale $\mu$ is due solely to the missing terms of $\mathcal{O}\left(\alpha_{s}^{n>2}\right)$. Since $\mu$ is just an arbitrary artifact of dimensional regularization, perturbation theory does not tell us what scale to choose, except for very tenuous hints.

The only thing that the theory tells us about $\mu$ is that the renormalization scale should be of the order of the $Q^{2}$ of the process. This choice is not based on any physical criterion but on the fact that it guarantees the smallness of terms of the order $\mathcal{O}\left(\left(\alpha_{s} \ln \left(\mu^{2} / Q^{2}\right)\right)^{N}\right)$ to all orders in the perturbative expansion [34].

In order to fit for $\Lambda_{\overline{M S}}$, and hence $\alpha_{s}$, we must choose a scale $\mu$. The freedom we have in choosing this scale we will call renormalization scale ambiguity and will be a source of systematic uncertainties in our measurement. We will estimate this uncertainty by fitting the data with two different conditions for $\mu$ : we treat $\mu$ both as a fixed $\left(\mu=M_{Z^{\circ}}\right)$ parameter and as a free parameter that optimizes the fit quality. From now on we will adopt the widely used definition $f \equiv \mu^{2} / M_{Z^{0}}^{2}$ for the renormalization scale used in the fit.
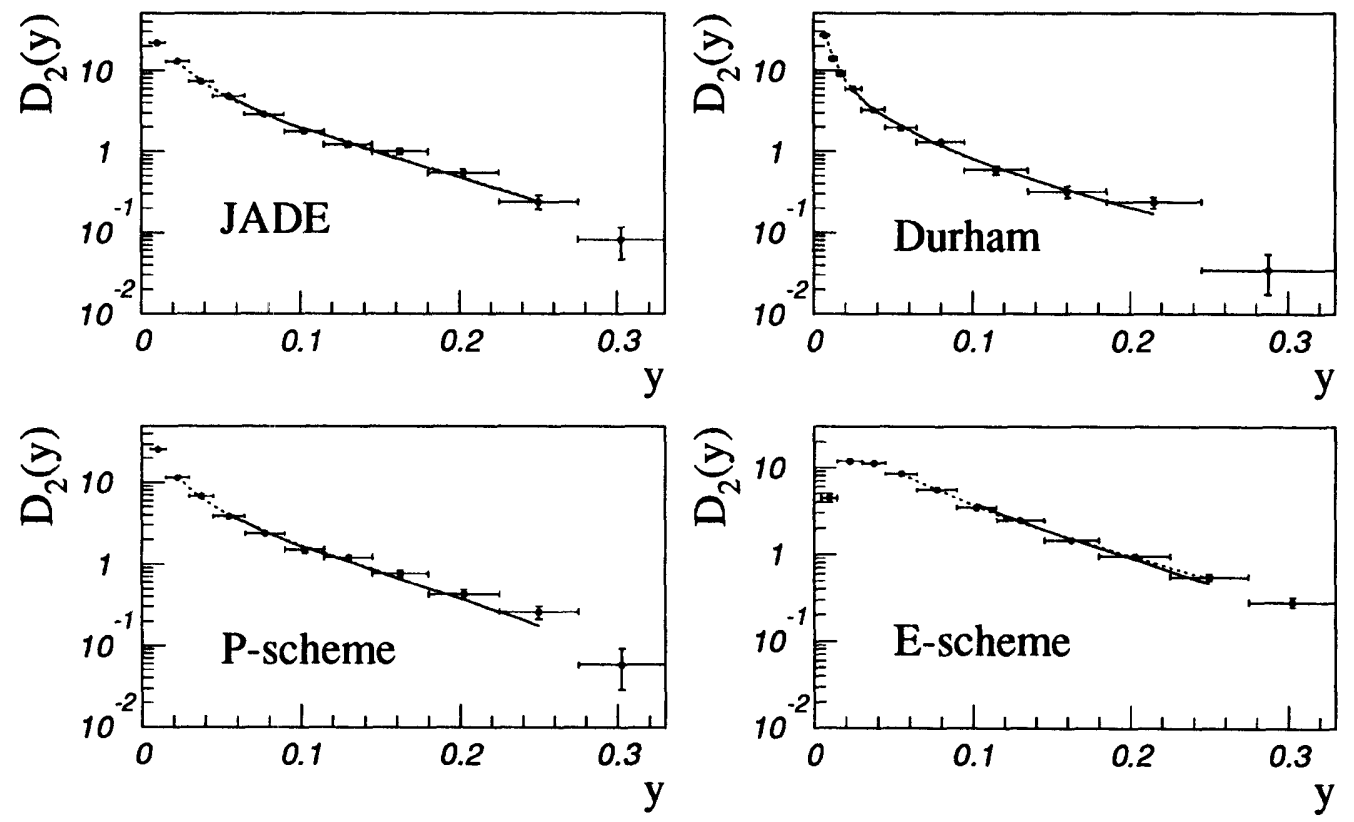

Figure 6-13: Results of the fits to $\Lambda \overline{M S}$. The points are the data, the solid line corresponds to the $f=1.0$ fit, and the dotted line corresponds to the fit with $f$ as a free parameter. Both the data and the fit are shown at the hadron level. 
Fit with $\mu^{2}=M_{Z^{0}}^{2}$

The characteristic energy scale in our experiment is undoubtedly $M_{Z^{0}}$. However, the radiation of gluons (with a factor of $\sqrt{\alpha_{s}}$ at its vertex) occurs at a lower $Q^{2} \lesssim M_{Z^{0}}^{2}$. For the purpose of investigating the scale dependence we used, as an upper bound, $f=1.0$.

The fit with $f=1.0$ is performed in the region where $R_{\geq 4} \leq 1 \%$. This condition is based on the observation $[51,52]$ at lower energies that the 4 -jet rate is not described properly by the $\mathcal{O}\left(\alpha_{s}^{2}\right)$ calculations. This is a somewhat circular argument but we will see in Section 6.7 that the same is true in our case. We then select the lower fit range using this requirement and reading off the proper bin ranges from Tables 6.1 to 6.4. The chosen bin ranges are included Table 6.6 along with the result of the one parameter fit to $\Lambda_{\overline{M S}}$. The fraction of events (in \% of 9878) included in the fit are presented in the 'fit range' column in parenthesis. The errors and the $\chi^{2}$ returned by MINUIT are also included in the table. The fit (at the hadron level) using the four jet algorithms is also presented in Figure 6-13 as a solid line.

\section{Fit with $\mu^{2}$ as a Free Parameter}

Since the renormalization scale is arbitrary but sensitive to the uncalculated higher order terms, a fit to the data with $\mu$ as a free parameter is an attempt to let this sensitivity determine the best fit. In this case, the fit range was chosen so that $R_{\geq 5} \leq 1 \%$ and that the perturbative calculations give sensible results (positive jet rates given for $y_{\text {cut }} \gtrsim 0.01$ ). We exclude the five and higher jet regions since we deal with at most four partons. Since we did not tabulate $R_{\geq 5}$, we determine this lower fit range from Figure 6-5. The results for this fit are also presented in the lower rows of Table 6.6 and in Figure 6-13 as the dashed line.

As in the fixed $f$ fit, the errors on $\Lambda_{\overline{M S}}$ and $f$, and the $\chi^{2}$ are the ones given by MINUIT. It is interesting to note the rather large variations in relative error for $f$. This is simply due to the sensitivity of the $\chi^{2}$ to the renormalization scale (as shown for the JADE algorithm in Figure 6-17).

The values of $\alpha_{s}$ are then calculated by using the fit values from Table 6.6 for $f$ fixed and free in Equation 2.15. The errors are propagated in the equation and, in the case of the two-parameter fit, the inter-parameter correlation term taken into account.

\subsection{Systematic Effects}

By "systematic effects" we mean all the biases introduced in the course of measurement that effectively shift the measurement values in an unknown way. Here we can classify them as either experimental (having to do with the mechanics of extracting the measurement) or theoretical (having to do with the validity of the ideas behind the 
measurement). What follows is a discussion of both type of effects. We will express the magnitude of each contribution in terms of $\alpha_{s}$ and give a detailed accounting of the breakdown of the contributions for the JADE algorithm. All of the contributions for the different algorithms are combined separately and presented in Table 6.7.

The estimation of systematic uncertainties is almost arbitrary. It is important to remember that most systematic contributions are not statistical in nature and reflect a true lack of knowledge about a particular process. In the following we will vary models, parameters, constants, and cuts within reasonable ranges in order to estimate their contributions to the systematic errors.

For each possible systematic effect considered in the next two sections, the full $\alpha_{s}$ analysis was carried out. The variation in the numerical value of $\alpha_{s}$ (beyond purely statistical effects) was taken to be the systematic contribution. Variations were studied to every entry tabulated in Table 6.6; the fixed $f=1.0$ fit and the free $f$ fit were considered separately and combined later.

\subsubsection{Experimental Systematics}

We investigate the experimental uncertainties by studying the effects of the detector and measurement procedure on the value of $\alpha_{s}$. From now on, we will call these errors instrumental uncertainties reserving the term experimental for the combination of statistical errors and instrumental errors. Since many of the distributions varied are on the edges of phase space (e.g., $N_{\text {clus }}$ ) the following errors are in general quoted as asymmetric errors. At the end, each contribution is added in quadrature and quoted in Table 6.7 as a single instrumental error.

\section{- Longitudinal Energy Deposition}

As pointed out in Section 6.2.2, the optimum jet resolution is obtained when we use the first two longitudinal layers of the calorimeter. Omitting any of the calorimeter layers in the analysis may cause (or aggravate) a bias by cutting short the jet development. In addition, a "sick" layer may unnecessarily merge or split jets. To investigate these effects the complete analysis was carried out (including correction factors) for the electromagnetic (EM) layer alone, for the EM and hadronic (HAD) layers alone and for the EM + HAD + WIC layers. The observed contribution to the systematic uncertainty was $\Delta \alpha_{s}={ }_{-0.0013}^{+0.005}$.

\section{- Relative Energy Calibration}

We discussed the longitudinal energy calibration of the calorimeter in Chapter 2. In order to study the effects of energy calibration errors the analysis was performed with two different sets of calibration factors. The $\lambda$ factors from Table 4.2 were varied by $\pm 5 \%$ and the combinations $0.95 \lambda_{\text {em }}+1.05 \lambda_{\text {had }}$ and 
$1.05 \lambda_{\text {em }}+0.95 \lambda_{\text {had }}$ used ${ }^{6}$ to reanalyze the data. A $5 \%$ variation in the energy scale is consistent with the measurement errors included in Table 4.2. The effect on $\alpha_{s}$ of this variation is very small: $\Delta \alpha_{s}= \pm 0.0002$.

\section{- Event Selection}

In order to investigate the effects of event selection the analysis was repeated with a "loose" and a "tight" set of selection cuts. The three event selection variables $\left(E_{v i s}, E_{i m b}\right.$, and $\left.N_{\text {clus }}\right)$ are varied by $\pm 5 \%$. This $5 \%$ variation in the selection cuts translates into an event yield uncertainty of about 200 events, consistent with the calculated yield uncertainty of 150 events (See Section 5.6). The systematic uncertainty due to this source is $\Delta \alpha_{s}= \pm_{0.0009}^{0.0004}$.

\section{- Time-dependent Effects}

In order to investigate any possible time dependent effects (e.g. time-dependent online calibration), the data-set was divided in thirty time intervals. For each interval the average differential jet-rate was calculated and plotted in Figure 614. Each observable, $\bar{y}_{3}, \bar{y}_{4}$, and $\bar{y}_{5}$ was then fit to a straight line. The results of the fit are consistent with a line of zero slope $\left(\chi^{2} / \mathrm{NdF}\right.$ below 1.5 for the three observables); we thus assign no systematic uncertainty contribution due to time-dependent effects.

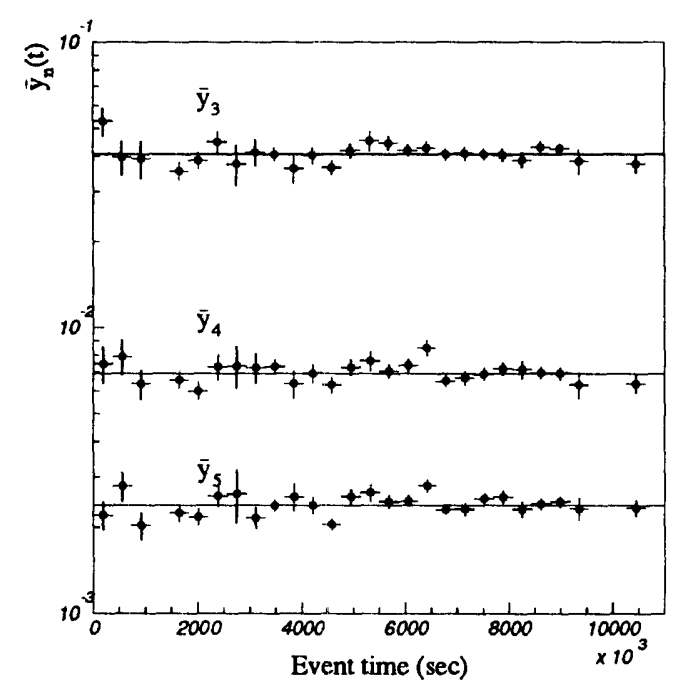

Figure 6-14: The average differential jet rates $\left(\bar{y}_{3}, \bar{y}_{4}\right.$, and $\left.\bar{y}_{5}\right)$ as a function of the event time. The zero-point corresponds to the start of the run. The lines shown are fits to a straight line.
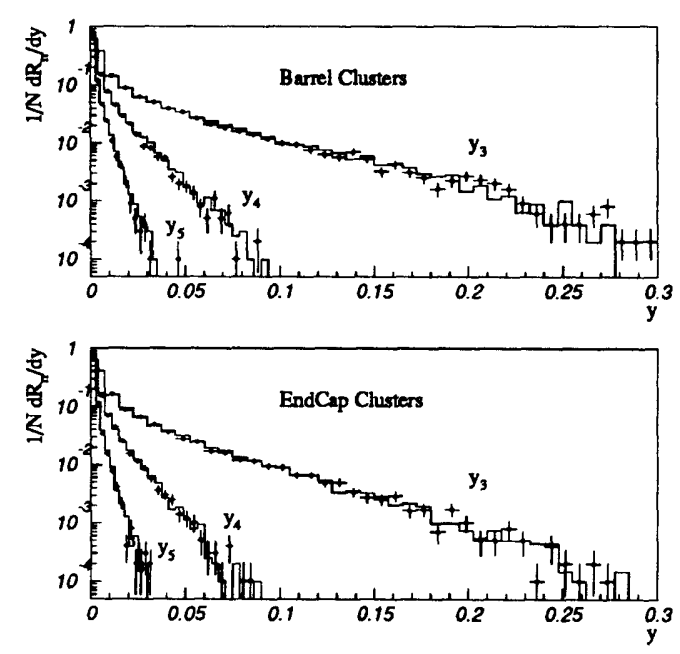

Figure 6-15: The differential jet rates using EndCap $\left(\left|\cos \theta_{\text {cluster }}\right| \geq 0.55\right)$ or Barrel $\left(\left|\cos \theta_{\text {cluster }}\right|<0.55\right)$ clusters only. The line (dots) correspond to the data (Monte Carlo).

\footnotetext{
${ }^{6}$ The combinations $1.05 \lambda_{e m}+1.05 \lambda_{h a d}$ and $0.95 \lambda_{e m}+0.95 \lambda_{\text {had }}$ are effectively just overall scale factors and do not affect the event topology.
} 


\section{- EndCap/Barrel Effects}

Since the energy response of the barrel and the endcap are quite different, in order to investigate possible biases, the analysis was carried out using barrelonly and endcap-only clusters. Figure 6-15 shows the result of the analysis for the differential jet-rate observables. A very good agreement between the data and the Monte Carlo is evident. This agreement assures us that any possible bias will be accounted for in the correction factors. Nevertheless, the variation in $\alpha_{s}$ due to the analysis of barrel-only clusters is $\Delta \alpha_{s}=+0.0002$. The endcaponly events were not analyzed because the phase space for 3 -jet events in the endcap-to-endcap region is very small and we have a relatively small event sample.

\section{- Cluster Selection}

The actual selection of clusters was varied in order to investigate any possible cluster energy threshold effects. To this end, the minimum cluster energy requirement was loosened to $E_{\text {clus }} \geq 0.100 \mathrm{GeV}$ and tightened to $E_{\text {clus }} \geq 0.250$ $\mathrm{GeV}$ (see Section 3.5 for more details). The complete analysis chain was again carried out and shifts of $\Delta \alpha_{s}={ }_{-0.0009}^{0.0008}$ were observed for the JADE algorithm. This large effect is not unexpected since low energy clusters are very sensitive to hit energy threshold and clustering effects. We have already remarked in Chapter 4 that this region is difficult to simulate and tune for a calorimeter.

\section{- Fit Range}

Another possible source of systematic error arises from choosing a fit range. The theoretical and experimental issues that motivated our choice for a fit range in the previous section may bias the determination of $\Lambda_{\overline{M S}}$ at the edges of the chosen phase space. For example, we know that the $\mathcal{O}\left(\alpha_{s}^{2}\right)$ calculations do not predict more than four partons and yet the data contains five-jet events in the low- $y$ region. In order to deal with that issue, we excluded events with $R_{\geq 5} \geq 1 \%$ in the two-parameter fits for $\Lambda_{\overline{M S}}$. The uncertainty associated with this choice enters into the 'fit range' uncertainty.

In order to estimate this uncertainty, both the fixed and free renormalization scale fits were repeated with the rightmost and leftmost data bins excluded. If by deleting or adding such a bin the $\chi^{2}$ per degree of freedom increased by more than one unit, then the systematic error was enlarged to accommodate this change. The uncertainties in $\alpha_{s}$ for the JADE algorithm were $\Delta \alpha_{s} \pm 0.0011$.

\subsubsection{Theoretical Systematics}

The theoretical systematic uncertainties serve as a survey of the aspects of the theory which are either not included in the $\mathcal{O}\left(\alpha_{s}^{2}\right)$ calculations or not well understood. For example, the fragmentation process is well understood only in terms of purely phenomenological models (and thus our need for Monte Carlo simulations). These 
models, as discussed in Section 2.4.2, are fairly sophisticated and reproduce the data quite well over a wide range of $Q^{2}$. However, many such models can in principle be constructed to give a reasonable description of the observations, in an almost "black-box" approach. Not being able to confirm precisely these predictions in detail means that we have to assign a systematic error due to hadronization when using a hadronization model.

The theoretical systematic uncertainties are also quoted as asymmetric errors. Since each contribution is relatively large, the individual contributions below have been listed separately in table 6.7. An essential reference to this section is Chapter 2, where the physics of the following systematic effects is covered in more detail.

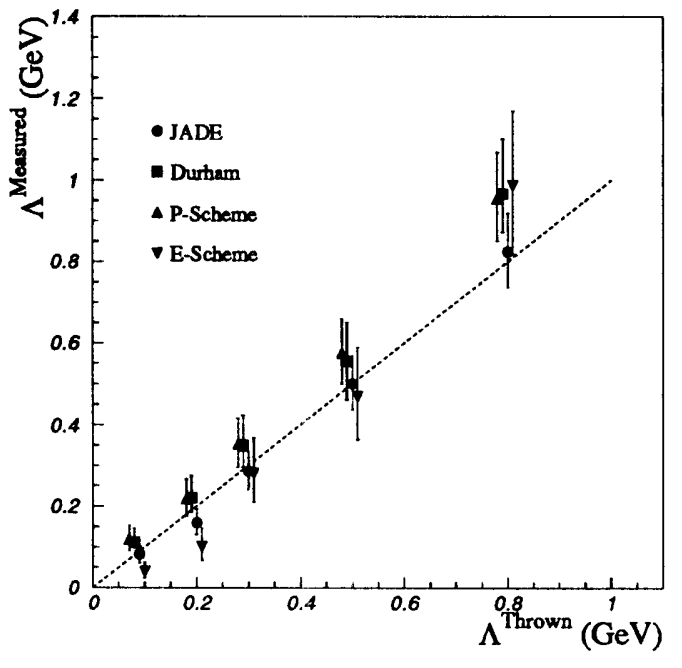

Figure 6-16: Result of test experiment (See Appendix B) for the four different algorithms. The dashed line corresponds to $\Lambda \frac{\text { thrown }}{M S}=\Lambda \frac{\text { measured }}{M S}$

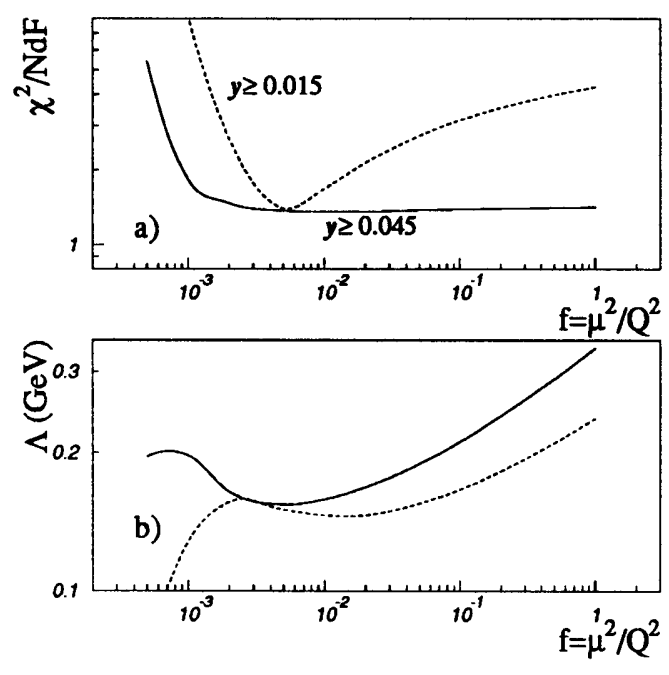

Figure 6-17: Sensitivity of the JADE algorithm to the scale $f:$ a) the reduced $\chi^{2}$ and b) $\Lambda_{\overline{M S}}$.

\section{- Renormalization Scale}

Since the theory does not tell us at what scale to perform the renormalization subtraction, other than "about the $Q^{2}$ of the process in question" we have almost complete freedom in choosing the renormalization scale. As seen in Equation 2.5 the truncation of the perturbative series introduces a $\mu$-dependence in the calculated quantities. In a sense, then, this dependence is a measure of the higher order missing terms.

In estimating the renormalization scale uncertainty we take the conservative approach of taking the difference in the $\alpha_{s}$ values at the scale $Q^{2}=M_{Z}^{2}$ and at the fitted scale $Q^{2}=f M_{Z}^{2}$. This is the largest of the systematic uncertainties as can be seen from the large differences in $\Lambda_{\overline{M S}}$ in Table 6.6. The quoted value 
of $\alpha_{s}$ in Table 6.7 is calculated as,

$$
\alpha_{s}=\frac{\alpha_{s}(f=1)+\alpha_{s}\left(f=\mu^{2} / M_{Z}^{2}\right)}{2}
$$

with the scale uncertainty,

$$
\Delta \alpha_{s}=\frac{\left|\alpha_{s}(f=1)-\alpha_{s}\left(f=\mu^{2} / M_{Z}^{2}\right)\right|}{2}
$$

where the $\alpha_{s}$ values are calculated from Equation 2.15 using the $\Lambda_{\overline{M S}}$ values from Table 6.6. The scale uncertainties are also summarized in Table 6.7.

Figure 6-17b shows the dependence of the fitted $\Lambda_{\overline{M S}}$ on the renormalization scale $f$ for the two fit ranges used. The $\chi^{2}$ per degree of freedom is shown in Figure $6-17 \mathrm{a}$ for the same $f$ range. It is clear that when the low- $y$ regions are included in the fit, a preference for small renormalization scales emerges.

\section{- Recombination Scheme}

Table 6.7 includes the results of the fits for the four jet algorithms used. We argued in Section 6.3.2 that the E-scheme algorithm would not be used as part of the measurement due to the large hadronization corrections. In addition, as is evident from the table, this algorithm also suffers from great sensitivity to the renormalization scale - yet another unattractive feature.

In order to test how well the different algorithms reconstruct $\alpha_{s}$, a similar test experiment to the one presented in Appendix $B$ is performed. In this case the "test variables" are the four algorithms. The results of $\Lambda_{\text {thrown }}$ versus $\Lambda_{\text {measured }}$ are shown in Figure 6-16. It is evident ${ }^{7}$ that the worst algorithm is the E-scheme, while the JADE scheme appears to have the least bias in reconstructing $\Lambda_{\overline{M S}}$.

The approach we employ in combining the three algorithm results is the same we took for the renormalization scale. Since the measurements are dominated by systematic errors, which are not derived from a normal distribution, it is appropriate to combine them in an unweighted fashion. We quote as as our final result the arithmetic average of the three numbers and quote the recombination scheme uncertainty as one-half of the maximum deviation from the three numbers. Using the values from Table 6.7, we find that the recombination scheme uncertainty then contributes $\Delta \alpha_{s}= \pm 0.003$ to the systematic error and yields a central value of $\alpha_{s}=0.122$.

\section{- Prefragmentation}

By prefragmentation we mean the stage between primary $q \bar{q}$ production and hadronization, represented by the first stage (for both parton shower and matrix

\footnotetext{
${ }^{7}$ One should take Figure 6-16 as a qualitative guide to the relative merits of the algorithms and not as a real measure of the systematic error. The reason is that the event generator used in the test experiment was limited to a matrix element calculation with $f=1$.
} 
element models) in Figure 2-6. In estimating the systematic contributions from this stage of the Monte Carlo, we investigated both the parton shower and the matrix element approach in the JETSET implementation.

For the JETSET ME study, 100,000 events were generated with a parton invariant mass cut-off of $y_{\text {cut }}=0.01$ and the JETSET default parameter set (See Chapter 2 for more details) for the $\mathcal{O}\left(\alpha_{s}^{2}\right)$ matrix element option. The average number of partons was $\left\langle N_{\text {parton }}\right\rangle=2.9$. As usual, after the partons were generated they were further fragmented into final state hadrons via the Lund string model (as implemented in JETSET) and the corresponding matrix $S(y)$ was applied to the data. This procedure provides a very conservative estimate of the prefragmentation uncertainty in the low- $y$ region where the missing higher order terms (i.e., more partons) are more important. This manifested itself generally in worse $\chi^{2}$ for the $\alpha_{s}$ fits with free $f$ and larger errors due to a reduced fit sensitivity to $f$.

As previously mentioned, the parton shower approach connects hadrons and primary partons by compensating missing higher orders with LLA AltarelliParisi parton branchings. The main parameter in this approach, apart from $\Lambda_{e f f}$, is the parton virtuality cut-off $Q_{0}$. We saw in Figure 2-5 the dependence of $\left\langle N_{\text {parton }}\right\rangle$ on $Q_{0}$. Most of the LEP experiments (e.g., $\left.[44,100,43]\right)$ have tuned the parton showers with $Q_{0} \approx 1.0 \mathrm{GeV}$. We also use $Q_{0}=1.0 \mathrm{GeV}$. In order to investigate the systematic effects of this cut-off, we vary $Q_{0}$ from 0.5 to $9.0 \mathrm{GeV}$ corresponding to $\left\langle N_{\text {parton }}\right\rangle=2.3$ to 9.0 respectively. We choose the upper limit to correspond with the same number of partons as in the matrix element approach above. It was found that above $Q_{0} \approx 6.0$ the reduced $\chi^{2}$ deteriorate significantly for both the fixed and free scale fits. For this reason the systematic contribution was estimated by: (1) assigning an error due to the largest variation in $\alpha_{s}$ from $Q_{0}=0.5-5.0 \mathrm{GeV}$ and (2) assigning an independent error due to the difference of the matrix element method and the parton shower method at $Q_{0}=9.0 \mathrm{GeV}$. This last error is an attempt to quantify the intrinsic differences of the parton shower/matrix element approaches in a 'level playing field' and is probably a very conservative method.

For the JADE algorithm, the uncertainty due to (1) is $\Delta \alpha_{s}={ }_{-0.003}^{0.001}$ and the one due to (2) is $\Delta \alpha_{s}=+0.001$. These uncertainties are combined in quadrature and summarized in Table 6.7.

\section{- HERWIG hadronization}

Even though the JETSET string fragmentation model with parton showers has been very successful in reproducing the QCD observables, other models provide reasonable predictions. HERWIG, discussed in Chapter 2, is an alternative QCD Monte Carlo based on a cluster model of hadronization. In HERWIG, the underlying scheme of producing partons is very similar to JETSET's parton shower approach (See Chapter 2). For this reason, the comparison of HERWIG with JETSET is mostly a comparison of their hadronization models. 


\begin{tabular}{||c|c|c|c|c|c|r|r||}
\hline \hline Scheme & $\alpha_{s}$ & $\begin{array}{c}\text { sta- } \\
\text { tistical }\end{array}$ & scale & $\begin{array}{c}\text { hadroni- } \\
\text { zation }\end{array}$ & $\begin{array}{c}\text { HER- } \\
\text { WIG }\end{array}$ & prefrag. & $\begin{array}{r}\text { instru- } \\
\text { mental }\end{array}$ \\
\hline JADE & 0.119 & \pm 0.003 & \pm 0.008 & \pm 0.001 & -0.002 & \pm 0.001 & \pm 0.002 \\
Durham & 0.125 & \pm 0.003 & \pm 0.006 & \pm 0.001 & +0.001 & \pm 0.004 & \pm 0.003 \\
P & 0.123 & \pm 0.003 & \pm 0.003 & \pm 0.001 & -0.002 & \pm 0.001 & \pm 0.004 \\
\pm 0.002 \\
E & 0.125 & \pm 0.004 & \pm 0.012 & \pm 0.002 & +0.003 & $\pm_{0.004}^{0.001}$ & \pm 0.002 \\
\hline
\end{tabular}

Table 6.7: Summary of the final results for the four algorithms with the corresponding systematic uncertainties. The value of $\alpha_{s}$ presented corresponds to the average value between the free and fixed scale fit; the scale error is one-half of the absolute difference between the two.

In order to compare the effects of a different hadronization model, the parton to hadron folding of Section 6.3.2 was repeated using the HERWIG Monte Carlo, version 5.7. The hadronization folding matrix $S(y)$ was obtained by generating 100,000 HERWIG events with the default parameters. Since the statistical effects of the folding matrix are negligible, we assign the net change in $\alpha_{s}$ as a systematic error due to hadronization model. We take the conservative approach and assign symmetric errors. The results are shown in Table 6.7.

\section{- Hadronization Parameters}

In order to investigate the sensitivity of the value of $\alpha_{s}$ to the hadronization parameters, the main JETSET fragmentation parameters were varied within the errors given in Table 2.1. A total of 5 different data sets were generated with the L3-tuned (See Chapter 2) parameters varied within their errors. Each set consisted of 100,000 events. The corresponding hadronization folding matrices were then obtained, applied in the fit to the data, and the variation in the resultant $\alpha_{s}$ recorded as a systematic effect. In addition, a completely different JETSET parameter set from TASSO (also in Table 2.1) was used in the folding as a further check. The TASSO-tuned parameter set [52] was obtained at $\sqrt{s}=$ $35 \mathrm{GeV}$. The results are summarized in Table 6.7.

\subsection{Results}

In the previous section we identified the sources of systematic errors and defined the strategy to follow in combining the measurements of Table 6.7 into a single final number.

As a central value of $\alpha_{s}$ at $Q^{2}=M_{Z}^{2}$ we quote the arithmetic average of the values for the JADE, Durham, P-scheme algorithms, namely,

$$
\alpha_{s}\left(M_{Z}\right)=0.122,
$$


and assign a recombination scheme uncertainty of \pm 0.003 . It is the nature of most systematic uncertainties (at least the ones encountered in this analysis) that they don't derive from a normal distribution and it is thus meaningless, perhaps even dangerous, to do a weighted average. In this case, this observation is especially important since the systematic errors are much larger than the statistical errors.

Following the previous discussion one may argue that there is no justification in combining the uncertainties in Table 6.7 into a single number. We will take a compromise approach by combining the theoretical and experimental uncertainties separately. In addition, we have the further complication of somehow "averaging" the errors of the three different algorithms.

This last complication is resolved in the following way. The statistical error of the resultant value is quoted as $\Delta \alpha_{s}=0.003$ (the same as each individual algorithm) since the three algorithms deal with the same data sample. For the case of the theoretical errors, we first combine them in quadrature for each separate algorithm. We take the moderate approach of then choosing as a final theoretical uncertainty the average of the three uncertainty values. This corresponds to the reasonable assumption that combining the three algorithms will give us a somewhat improved measurement but not necessarily the best measurement. We apply the same procedure to the instrumental error. The result is thus,

$$
\alpha_{s}\left(M_{Z^{0}}\right)=0.122 \pm 0.004_{0.006}^{0.007} \pm 0.003,
$$

where the quoted uncertainties are the experimental, theoretical, and recombination scheme uncertainties respectively. Further combining the last two into a single theoretical error then yields,

$$
\alpha_{s}\left(M_{Z^{0}}\right)=0.122 \pm 0.004_{-0.007}^{0.008}
$$

which we quote as our final result. One may further combine the experimental and theoretical uncertainties into a single error:

$$
\alpha_{s}\left(M_{Z^{0}}\right)=0.122 \pm 0.008 \text {. }
$$

\subsection{Discussion}

So far we have measured $\alpha_{s}$ at $\sqrt{s}=91.6 \mathrm{GeV}$ and investigated the different sources of systematic uncertainties. We have seen that even with just under 10,000 events, the measurement errors are dominated by theoretical uncertainties. These uncertainties are in turn dominated by the truncation of the perturbative series, which introduces an unphysical dependence on the renormalization scale.

In Chapter 2 we briefly mentioned various theoretical prescriptions to resolve the scale ambiguity. These schemes offer recipes to quote measurements in a way that either minimizes or standardizes the effects of the missing higher order terms. On 


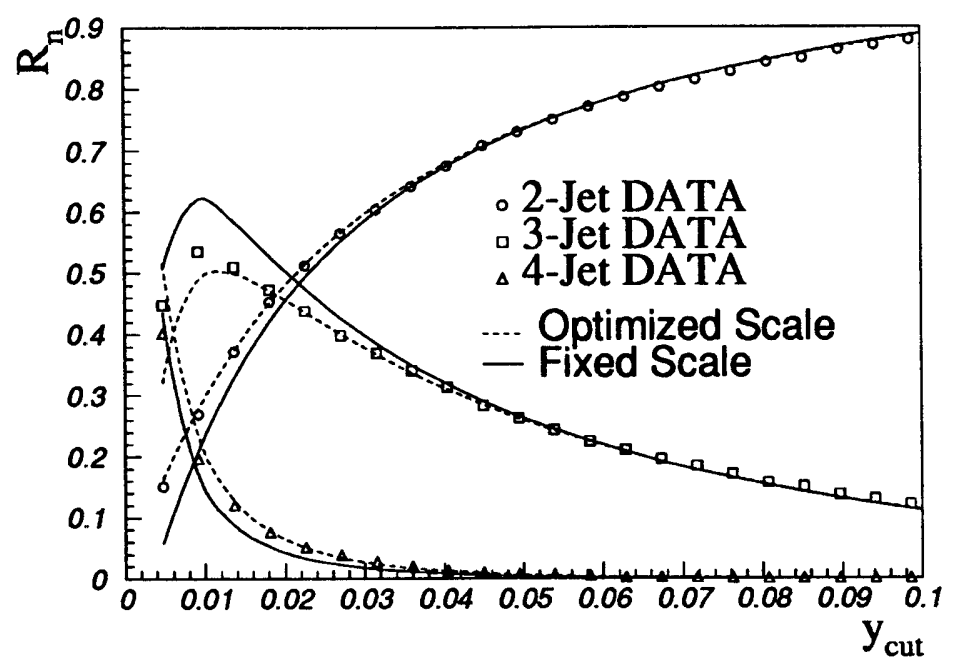

Figure 6-18: Detail of the corrected JADE jet cross section with the fixed and optimized scale fits. Notice that the $f=1.0$ fit does a poor job for $y \lesssim 0.05$.

the surface these techniques have reasonable requirements. However, the inescapable truth is that there is no substitute for the missing terms; these prescriptions are thus conventions rooted on an analysis of the calculational mechanics of perturbative QCD, with generally very little physical motivation. There are always unpredictable higher order terms that "... cannot be anticipated by any magical procedure" [101]. We thus steer clear of these methods.

\section{Kinematics and the Renormalization Scale}

The previous discussion does not imply that there is no judicious choice of scale. In fact, the choice $\mu \approx Q$, where $Q$ is the characteristic energy of the process, is the appropriate one in order to avoid large $\ln (\mu / Q)$ terms in the perturbative expansion. With this in mind we can then ask, what is a sensible $Q^{2}$ for our jet analysis? Clearly $f=1.0$ is not the appropriate scale since radiated gluons have a bremsstrahlung-like spectrum with $Q^{2} \lesssim(45 \mathrm{GeV})^{2}$. One can argue that the scale of interest in such a case ought to be the momentum transfer at the quark-gluon splitting. In fact, we have indirect evidence for this in Figures 6-18 and 6-17a.

Figure 6-18 shows the result of the one and the two parameter fit to the data. We recall that the fixed scale fit was performed for $y \geq 0.045$ and the free scale fit was carried out for $y \geq 0.015$. We further recall that a low-y 3-jet event implies a soft gluon jet. From this, we can make three inferences:

1. The $\chi^{2}$ preference for a small renormalization scale when the low-y region is included in the fit is due to the fact that the calculations do not reproduce the 4jet rate (cf, Figure 6-18). The missing higher orders terms are then compensated 


\begin{tabular}{||c|c|c|r||}
\hline \hline Scheme & $\Lambda \frac{(5)}{M S}$ & fit range & $\chi^{2} / N d F$ \\
\hline \multirow{2}{*}{ JADE } & $206_{-29}^{+32}$ & $0.045-0.25(35)$ & $9.6 / 6$ \\
& $167_{-22}^{24}$ & $0.015-0.25(62)$ & $17.4 / 7$ \\
\hline \multirow{2}{*}{ Durham } & $249_{-41}^{+45}$ & $0.030-0.20(18)$ & $6.0 / 6$ \\
& $260_{-28}^{+33}$ & $0.005-0.20(51)$ & $8.8 / 8$ \\
\hline \multirow{2}{*}{$\mathrm{P}$} & $236_{-39}^{ \pm 42}$ & $0.045-0.25(27)$ & $10 . / 6$ \\
& $268_{-33}^{36}$ & $0.015-0.25(55)$ & $12.2 / 7$ \\
\hline \multirow{2}{*}{$\mathrm{E}$} & $248_{-69}^{+55}$ & $0.090-0.25(29)$ & $13 . / 4$ \\
& $212_{-44}^{48}$ & $0.045-0.25(60)$ & $19 . / 5$ \\
\hline \hline
\end{tabular}

Table 6.8: Results of the fits with $\mu^{2}=y_{3} E_{c m}^{2}$. Units are in MeV. The numbers in parentheses in the 'fit range' column are the fraction, in \%, of the 9,878 total events contained in the fit range. Each algorithm has two results for two different fit ranges (third column).

by a small scale $f$.

2. Events with hard gluons $(y \gtrsim 0.05)$ show no such preference (cf., Figure 6-17a) and are well described by the $\mathcal{O}\left(\alpha_{s}^{2}\right)$ calculations.

3. The free- $f$ fit fails to describe the $y \lesssim 0.015$ region where $R_{5}$ is not negligible (as determined for Figure 6-5).

In light of the above, we can try to postulate a renormalization scale based on kinematics alone [40]. One possible scheme is to use the energy transfer to the gluon jet. If we think of a three parton configuration with the gluon being one of the two lowest energy partons, then the $Q^{2}$ of the process is,

$$
Q^{2} \approx y_{3} E_{c m}^{2},
$$

where $y_{3}$ (our usual definition) is the minimum scaled invariant mass for the system. If we use the above scale in our fits, by setting $\mu^{2}=y_{3} E_{c m}^{2}$, we obtain the results presented in Table 6.8. For these fits, the same bin ranges of Table 6.6 were used.

We can see that these results produce consistent values of $\Lambda_{\overline{M S}}$ for both the low and the high-y region (cf., Table 6.6). However the fit $\chi^{2}$ values are consistently worse for the low- $y$ fit; not an unexpected result since we are no long optimizing the scale.

Using the same procedure that we used above to obtain a final result, we obtain a central value of

$$
\alpha_{s}=0.119,
$$

with a recombination scheme uncertainty of $\Delta \alpha_{s}=0.003$. By construction, we no 
longer have a renormalization scale uncertainty, however, an additional uncertainty of $\Delta \alpha_{s}=0.002$ would have to be included in the result to make the $\chi^{2}$ values compatible with those of Table 6.7. We see that by using a kinematics-based renormalization scale we get a result consistent with the final result in Equation 6.14.

\section{The Result in Terms of $\Lambda_{\overline{M S}}$}

We have said all along that the fundamental constant in $Q C D^{8}$ is $\Lambda_{\overline{M S}}$ and yet we have avoided so far quoting a number for it. We can convert our final (single error) result into a measurement of $\Lambda_{\overline{M S}}$ by using the alternate solution to the $\beta$-function in Equation 2.16. This yields, including experimental and theoretical uncertainties,

$$
\Lambda_{\overline{M S}}=0.28 \pm 0.10 \mathrm{GeV}
$$

\subsubsection{Running of $\alpha_{s}$}

The measurement of the three-jet rate $R_{3}$ at a fixed $y=y_{\text {cut }}$ as a function of the center-of-mass energy is a direct measure of the energy dependence of hard gluon radiation. Since the rate of gluon radiation, to leading order, is proportional to $\alpha_{s}$ (see Equation 2.23), then the measurement of $R_{3}(\sqrt{s})$ is the natural place to study the energy-dependence of $\alpha_{s}$.

We proceed by collecting various measurements spanning PETRA, PEP, TRISTAN, LEP [102, 103, 52, 104, 105, 106, 107], and in our case, SLC. These measurements represent energies $\sqrt{s}=22-92 \mathrm{GeV}$ in $e^{+} e^{-}$machines. Before comparing experiments at different energies it is essential to agree on the same jet definition. Since the JADE algorithm was the first widely used algorithm, the results presented in Figure 6-19 are calculated with the JADE algorithm. In addition, the same jet resolution cut $y_{c}=0.08$ was used throughout. This ensures that hadronization effects are kept to a minimum, or equivalently, that one is only probing the "hard" part of an event. All the measurements, when necessary, have been corrected for detector effects to the hadron level by each experiment.

It is evident from the figure that the strong coupling runs as predicted (or constructed) by the non-Abelian nature of QCD. Our result from Table 6.6 for $\mu=M_{Z^{0}}$ is presented, corrected for hadronization, as a solid line in the figure. For comparison, the same result is presented without hadronization corrections with the dotted line. The hadronization corrections were calculated with the JETSET parton shower Monte Carlo for the span of energy included in the figure. These corrections amount to $5-8 \%$ of the total 3 -jet rate.

In order to check for consistency with a single $\Lambda_{\overline{M S}}$, we proceed to fit the data in Figure 6-19 to the ERT calculation for $R_{3}$ (with hadronization corrections) for the

\footnotetext{
${ }^{8}$ In the approximation of massless quarks.
} 


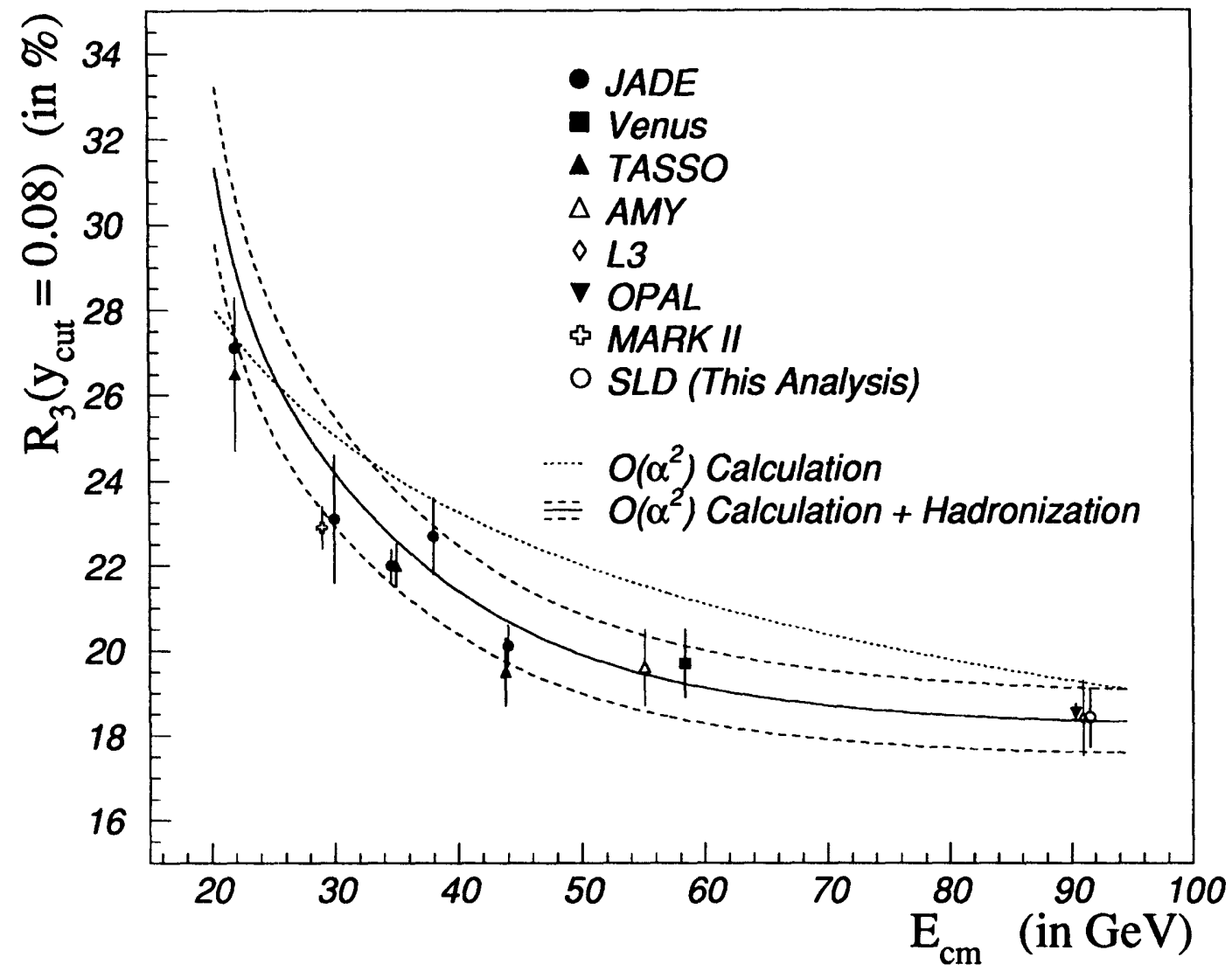

Figure 6-19: The energy dependence of the 3-jet fraction $\left(R_{3}\right)$ using the JADE algorithm at $y_{c u t}=0.08$. The data presented (see text for references) are corrected for detector effects only. The prediction of the ERT $\mathcal{O}\left(\alpha_{s}^{2}\right)$ calculations are also presented for the measured value of $\Lambda_{\overline{M S}}$ from Table 6.6.

$\Lambda_{\overline{M S}}$ parameter. The result of this one parameter fit is $\Lambda_{\overline{M S}}=0.340 \pm 0.010$ with a $\chi^{2}$ of 19 for 13 degrees of freedom. This confirms that, in the energy range covered in the figure, the strong coupling decreases with energy as predicted by QCD.

We should point out that in this case the renormalization scale ambiguity plays very little role because we are just interested in the evolution of $R_{3}$. Also, a two parameter fit was not carried out because at $y_{c}=0.08$ the $\chi^{2}$ shows no sensible minimum (cf., Figure 6-17a).

\subsubsection{Other Measurements of $\alpha_{s}$ and Final Thoughts}

In order to test QCD thoroughly it is necessary to study different processes at different $Q^{2}$. The last five years have been especially fruitful in this sense; inherent difficulties 
notwithstanding, QCD appears to be in solid ground, at least in the perturbative regime.

Clearly, one of the most unique aspects of QCD is the behavior of its $\beta$-function and the associated running of the strong coupling. The first evidence for this, and perhaps the most convincing [108] so far, came in with the observation of the scaling violations in deep inelastic scattering (DIS). Classical QCD does not predict such behavior. It is the quantized theory, with the coupling specified at a reference scale, that gives rise to the scaling violations. In more general terms, it is important to show that all QCD perturbatively calculable processes can be described by a single scale parameter $\Lambda_{\overline{M S}}$.

\begin{tabular}{||c|c|c|c|c|c||}
\hline \hline Experiment & Order & Ref. & $\mathrm{Q}(\mathrm{GeV})$ & $\alpha_{s}(Q)$ & $\alpha_{s}\left(M_{Z^{0}}\right)$ \\
\hline$R_{\tau}$ & $\mathcal{O}\left(\alpha_{s}^{3}\right)$ & {$[109]$} & 1.78 & $0.330 \pm 0.046$ & $0.118 \pm 0.005$ \\
$\Gamma\left(Z^{0} \rightarrow\right.$ had. $)$ & $\mathcal{O}\left(\alpha_{s}^{3}\right)$ & {$[5]$} & 91.2 & $0.132 \pm 0.012$ & $0.132 \pm 0.012$ \\
$p \bar{p} \rightarrow \mathrm{W}+$ jets & $\mathcal{O}\left(\alpha_{s}^{2}\right)$ & {$[110]$} & 80.6 & $0.123 \pm 0.027$ & $0.121 \pm 0.026$ \\
DIS $(\mu, \nu)$ & $\mathcal{O}\left(\alpha_{s}^{2}\right)$ & {$[5]$} & various & - & $0.112 \pm 0.007$ \\
Event Shapes & & & & & \\
OPAL & $\mathcal{O}\left(\alpha_{s}^{2}\right)$ & {$[100]$} & 91.2 & $0.124_{-0.008}^{+0.010}$ & $0.124_{-0.008}^{+0.010}$ \\
OPAL & resum. & {$[100]$} & 91.2 & $0.122_{0.003}^{0.003}$ & $0.122_{-0.006}^{0.003}$ \\
Jet Rates & & & & & \\
MARK II & $\mathcal{O}\left(\alpha_{s}^{2}\right)$ & {$[111]$} & 29.0 & $0.149 \pm 0.007$ & $0.122 \pm 0.005$ \\
SLD Tracking & $\mathcal{O}\left(\alpha_{s}^{2}\right)$ & {$[112]$} & 91.6 & $0.118 \pm 0.011$ & $0.118 \pm 0.011$ \\
OPAL & $\mathcal{O}\left(\alpha_{s}^{2}\right)$ & {$[100]$} & 91.2 & $0.122_{-0.006}^{0.007}$ & $0.122_{-0.006}^{+0.007}$ \\
This Analysis & $\mathcal{O}\left(\alpha_{s}^{2}\right)$ & - & 91.6 & $0.122 \pm 0.009$ & $0.122 \pm 0.009$ \\
\hline \hline
\end{tabular}

Table 6.9: Summary of some of the other $\alpha_{s}$ measurements. When the reference did not quote a single error, multiple errors were combined in quadrature. The Mark II results were translated to $\mu=M_{Z^{0}}$. This table is just a short sampling; extensive reviews are found in $[113,5]$ and references therein.

Table 6.9 presents a short summary of selected measurements of $\alpha_{s}$ for different processes and at different energy scales. The agreement between such different processes (including the result of this analysis) confirms our present belief that QCD is the theory of strong interactions. Other interesting measurements [114] have extracted, via likelihood fits to independent terms in the cross sections, the group constants for QCD.

The totally inclusive (and thus hadronization insensitive) DIS and $R_{\tau}$ (hadronic decay fraction of the $\tau$ lepton) measurements are especially suited [108] for determinations of $\alpha_{s}$. However, the clean center-of-mass environment of an $e^{+} e^{-}$collider is experimentally ideal for the study of multiparton final states (via jet rates, event shapes, etc). The inevitable hadronization model dependencies, although reduced at 
the higher energies, still plague these measurements. However, it is evident that in this case the major limitation in our measurements is due to the renormalization scale uncertainty, which in turn is due to an incomplete perturbative series (uncalculated higher-order terms). The proposed prescriptions to solve this last problem amount to conventions in quoting a result-there is very little physical basis. Only the calculation of higher orders in the perturbative series has the potential of reducing this problem.

When such techniques become available, the detector-corrected data in Table 6.5 can be fit directly to the improved cross sections. 


\section{Appendix A}

\section{Observables}

\section{A.1 Introduction}

In this appendix we will discuss the definitions and properties of some of the observables that have been used in this analysis.

\section{A.2 Thrust and Sphericity}

\section{Sphericity}

Sphericity was originally proposed in 1970 [31] as an observable sensitive to jet structure. It was later [32] used to demonstrate the existence of jets. Sphericity is defined from the quadrupole moment of the event momentum distribution using the eigenvalues of the momentum tensor,

$$
T_{\alpha \beta}=\left\langle\frac{\sum_{i} \frac{3}{2} p_{\alpha}^{i} p_{\beta}^{i}-\frac{1}{2} \delta_{\alpha \beta} p_{i}^{2}}{\sum_{i} p_{i}^{2}}\right\rangle
$$

where $i$ runs over all particles in an event and the brackets denote averaging over all events. The eigenvalues of the tensor $T_{\alpha \beta}$ are ordered, $\lambda_{1} \geq \lambda_{2} \geq \lambda_{3}$, and the sphericity is defined as:

$$
S=\frac{3}{2}\left(\lambda_{2}+\lambda_{3}\right)
$$

From a perturbative-QCD approach, sphericity is not a good observable because it is not collinear safe or infrared safe. Since the observable is quadratic in momentum, any splitting of a particle into two collinear particles yields a different result (e.g., $\vec{k}=\vec{k} / 2+\vec{k} / 2$, then $\left.k^{2} \rightarrow 2(k / 2)^{2}\right)$.

Another observable defined in terms of the momentum tensor is the aplanarity. It is defined as the maximum of the three tensor eigenvalues, i.e., 


$$
A=\frac{3}{2} \lambda_{3} .
$$

The aplanarity observable suffers from the same collinear and infrared problems as the sphericity.

\section{Thrust}

Thrust was proposed [115] as an infrared and collinear-safe alternative for a jet observable. Like sphericity, it uses the particle momentum (or energy) vectors averaged over the event sample,

$$
T=\max \frac{\sum_{i}\left|\vec{n} \cdot \vec{p}_{i}\right|}{\sum_{i}\left|\vec{p}_{i}\right|}
$$

where $\vec{n}$ lies on the axis that maximizes the sum $T$. The variable $T$ is called thrust and $\vec{n}$ is called the thrust axis. The value of thrust measures how ' 2 -jetty' an event is; $T=1$ corresponds to two completely back-to-back jets, while $T=0.5$ corresponds to an isotropic event.

\section{A.3 Kinematics of three jets}

Irrespective of how we group a set of particles into jets with a jet algorithm, we can always think of jets as vectors. Each jet-vector is, of course, a 'sum' of its individual constituent clusters or charged tracks. This 'sum' depends on the particular jet algorithm employed.

Here we concentrate on the kinematics of 3 jets since it is the $q \bar{q} g$ final state that we use for determining $\alpha_{s}$. We label our jets in decreasing order of energy: $E_{1} \geq E_{2} \geq E_{3}$. If we normalize these energies to the beam energy $\frac{1}{2} E_{c m}$, then,

$$
x_{1}+x_{2}+x_{3}=2
$$

where $x_{i}=2 E_{i} / E_{c m}$ and $x_{1} \geq x_{2} \geq x_{3}$. Equation A.5 is only valid for a three parton system where there is no "smearing" due to resolution effects, hadronization effects, and missing energy. If we apply the definition in Equation A.4 to Equation A.5 we obtain:

$$
T=x_{1} .
$$

In a real event the above equation is only a correlation due to the pseudo-cluster recombination effects in forming the jets (see Chapter 6).

It is convenient (for the purposes of the analysis) to rewrite the above in terms of normalized invariant masses. Denoting the partons' 4-vectors as $P_{i}$ we write,

$$
\begin{aligned}
\left(P_{1}+P_{2}+P_{3}\right)^{2} & =E_{c m}^{2} \\
& =2 P_{1} \cdot P_{2}+2 P_{1} \cdot P_{3}+2 P_{2} \cdot P_{3},
\end{aligned}
$$


where $P_{i}^{2}=0$ since we deal with massless partons. If we define,

$$
y_{i j} \equiv \frac{2 P_{i} \cdot P_{j}}{E_{c m}^{2}},
$$

then,

$$
y_{12}+y_{13}+y_{12}=1 \text {. }
$$

It is also useful to consider the identity,

$$
P_{1}+P_{2}=\left(E_{c m}, \overrightarrow{0}\right)-P_{3},
$$

and rewrite it, squaring both sides,

$$
2 P_{1} \cdot P_{2}=E_{c m}^{2}-2 E_{3} E_{c m} .
$$

Normalizing by $E_{c m}^{2}$ and using the above definitions, we obtain,

$$
y_{12}=1-x_{3} .
$$

The above equation can be easily generalized for the three partons by cycling the indices. It is interesting to note that if we combine Equations A.9 and A.6, we obtain,

$$
y_{23}=1-T,
$$

which is just what we call ' $y_{3}$ ' in Chapter 6 .

\section{A.4 Differential Jet Rates}

The jet rates have been defined in Chapter 6. In Equation 6.3 we also defined the $D_{2}$ distribution as a convenient alternative to the jet rates free of inter-bin correlations. We can also consider $D_{2}$ as the distribution of $y_{3}$, the point in $y$ at which a 3 -jet event turns into a 2-jet event in a particular algorithm.

\section{Calculating the Jet Rates}

All of the jet algorithms used in this analysis are based in an invariant mass resolution parameter $y$. The differences between them have already been described in Chapter 6 and have to do with the ways clusters are recombined and the invariant mass defined. The most important feature of all of these algorithms is in the way they proceed: all invariant mass combinations $y_{i j}=M_{i j}^{2} / E_{v i s}^{2}$ between all clusters are calculated, then ordered, and subsequently recombined until a resolution cutoff $y_{i j} \geq y_{\text {cut }}$ is reached.

It is helpful to consider the parton level situation where there is no smearing due to hadronization. To second order in perturbative QCD we can have at most four partons. If we apply our algorithm to this 4-parton system, we have to calculate 
$4 \cdot(4-1) / 2=6$ invariant mass combinations. If we define $y_{4}$ as:

$$
y_{4} \equiv \min \left\{y_{12}, y_{13}, y_{14}, y_{23}, y_{24}, y_{34}\right\}
$$

where we have labelled each parton as $1,2,3$, and 4 , we see that $y_{4}$ is the point in ' $y$-space' when a 4-jet event turns into a 3 -jet event. We can also define the analogue for a three parton system; $y_{3}$ is defined as being the minimum of the 3 invariant mass combinations in a 3 parton system. This scheme is ideal for calculating jet rates as it allows an exact calculation in terms of the parameter $y$; it is also the scheme used in the cross section calculations of ERT [39]. 


\section{Appendix B}

\section{Detector Correction Procedure}

\section{B.1 Introduction}

In this appendix we will describe the unfolding procedure used to correct the jet fractions for detector effects.

We call "detector effects" all the effects that smear the physical observables in our experiment. This smearing is caused mainly by the finite detector resolution (for both energy and position measurements) and by the limited detector acceptance. In this Appendix we discuss the two correction methods used, the inversion and the factor method, and compare their merits and drawbacks.

\section{B.2 The Inversion Method}

In the inversion method, the approach we take in correcting our measured quantities (jet-rates) for detector effects is to unfold the data. In practice we parameterize the smearing by a matrix of transition probabilities using the detector Monte Carlo description ${ }^{1}$. We then correct the data by applying the inverse of this transition matrix to the observed (i.e. "detector level") distributions so that we are left with the corrected (i.e. "hadron level") distributions.

In general, this inversion method [98] results in unstable solutions when applied to measurements characterized by large resolutions. The long range bin-to-bin migrations induce large fluctuations in the inverted matrix which in turn affect the unfolded distributions. Confronted with this situation, many experimenters choose to use a bin-by-bin correction method (to be discussed in the next section) which is stable but generally input model-dependent. The correct approach in using the inversion method is to choose the binning of the measured distribution to properly

\footnotetext{
${ }^{1}$ This is equivalent to determining the resolution and acceptance functions.
} 
reflect the intrinsic resolution. This approach minimizes the bin-to-bin migration, insures a stable unfolding, and greatly reduces any model dependent biases.

\section{Method Outline}

In our measurement we are concerned with jet fractions. We define the jet fraction as vectors:

$$
\vec{D}(y) \equiv\left(\begin{array}{c}
R_{2}^{d}(y) \\
R_{3}^{d}(y) \\
R_{\geq 4}^{d}(y)
\end{array}\right) \quad \vec{H}(y) \equiv\left(\begin{array}{c}
R_{2}^{h}(y) \\
R_{3}^{h}(y) \\
R_{\geq 4}^{h}(y)
\end{array}\right)
$$

where $R_{2}$ denotes the 2 jet rate, $R_{3}$ the 3 jet rate, and $R_{\geq 4}$ denotes the 4 and higher jet rate. The superscripts $d$ and $h$ denote that the rates have been calculated at the detector and the hadron level, respectively. These vectors are a function of the jet parameter $y$ (we will drop $y$ from our notation) and are constrained by:

$$
D_{1}+D_{2}+D_{3}=H_{1}+H_{2}+H_{3}=1
$$

We assume we can relate $\vec{D}$ and $\vec{H}$ by a linear transformation,

$$
\vec{D}=T \vec{H}
$$

where $T$ is in general a $y$-dependent $3 \times 3$ matrix. In our case we are interested in extracting the physics contained in $\vec{D}$; to do so we first determine $T$ from the detector simulation of acceptance and resolution. We do so by generating hadronic Monte Carlo events and simulating the detector response using GEANT3. For each event the jet algorithm is applied for all $y$, and at each $y$ the migration of events between different jet classifications is tabulated in the matrix $T$. The matrix $T$ is then defined as,

$$
T_{i j}=\frac{\text { number of } i \text {-jet events at the hadron level that look like } j \text {-jet }}{\text { total number of } i \text {-jet events at the hadron level }}
$$

Once this matrix is obtained and properly normalized, we invert it in order to unfold the data for detector effects. We apply the inverse to both sides of equation B.3:

$$
\vec{H}=T^{-1} \vec{D}
$$

and we are left with the hadron level jet-rates.

\section{Unfolding Errors}

Since we obtain the matrix $T$ from a finite Monte Carlo sample, the elements of $T$ have statistical errors associated with them. These errors are especially noticeable in the less populated regions of phase space (e.g. in the 4-jet rate at high $y$ ) and they induce large fluctuations in the inverse matrix. For this reason it is crucial to 
propagate the errors on $T^{-1}$ correctly.
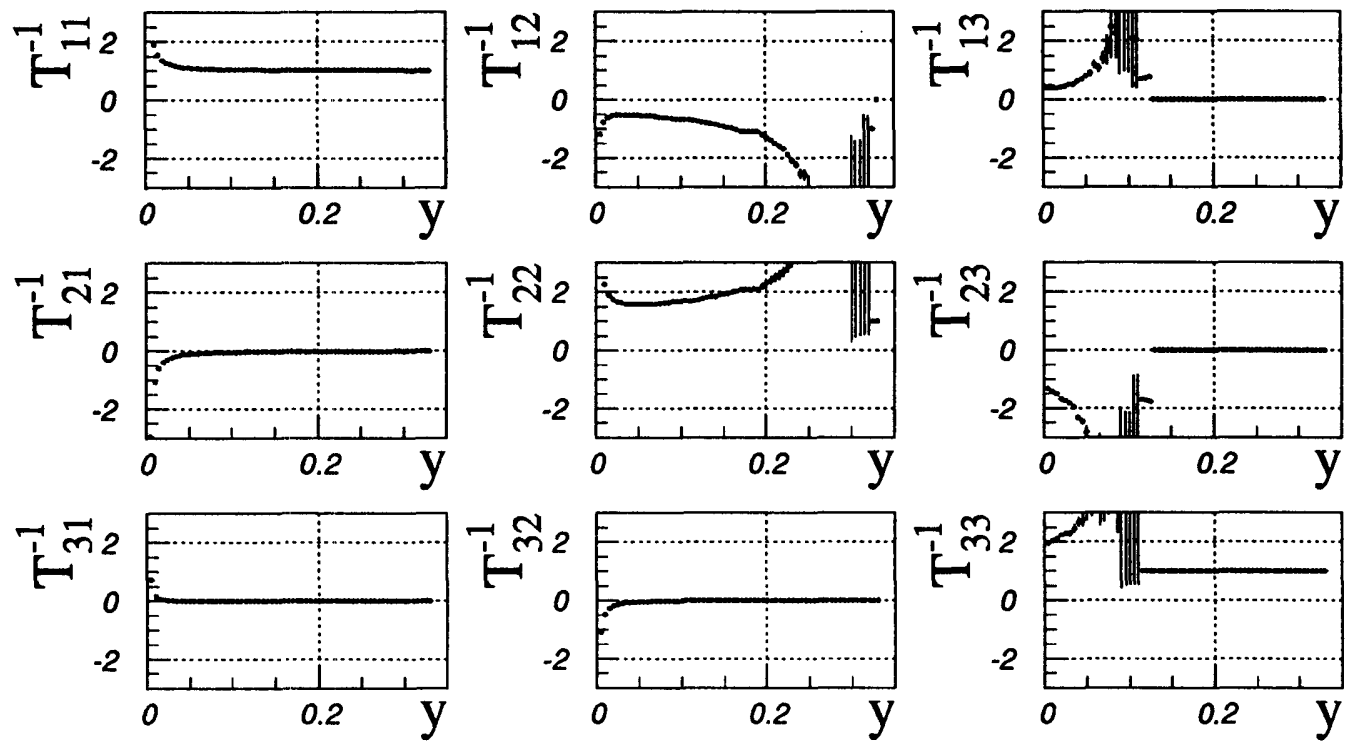

Figure B-1: The nine figures show the elements of the detector unfolding matrix $T^{-1}(y)$ described here. This matrix was calculated using JETSET 6.3 Parton Shower Monte Carlo with the JADE algorithm and a simulation of the SLD.

The error on the unfolded vector $\vec{H}$ due only to the inversion procedure may be written as [116]:

$$
\delta \vec{H}=\left(\delta T^{-1}\right) \vec{D} .
$$

Using the above, we can immediately write the covariance matrix on $\vec{H}$ :

$$
\begin{aligned}
\Sigma_{\mu \lambda} & =\overline{\delta H_{\mu} \delta H_{\lambda}} \\
& =\overline{\delta T_{\mu m}^{-1} \delta T_{\lambda n}^{-1}} D_{m} D_{n}
\end{aligned}
$$

where repeated indices imply summation. Using the identity $\delta T^{-1}=-T^{-1}(\delta T) T^{-1}$ we can rewrite equation B.5 as,

$$
\Sigma_{\mu \lambda}={\overline{\delta T_{l k} \delta T_{s t}}}_{\mu l}^{-1} T_{k m}^{-1} T_{\lambda s}^{-1} T_{t n}^{-1} D_{m} D_{n}
$$

The constraint

$$
\sum_{l} T_{l k}=1
$$


follows from equation B.4 and the unitarity condition (no events are created or destroyed by our correction procedure). We use this constraint, and the fact that there are no inter-column correlations in the matrix $T$, to write down the correlation term of equation B.8:

$$
{\overline{\delta T_{l k} \delta T_{s t}}}_{=} \frac{T_{l k}}{N H_{k}}\left(\delta_{l s}-T_{s t}\right) \delta_{k t}
$$

where the $\delta$ are the Kronecker delta and $N$ is the total number of events in the Monte Carlo sample. Putting equation B.10 into equation B.8, collecting terms and simplifying, we obtain:

$$
\Sigma_{\mu \lambda}=\frac{1}{N}\left(T_{\mu l}^{-1} T_{k m}^{-1} T_{\lambda l}^{-1} T_{k n}^{-1} T_{m s} T_{n t} T_{l k} \frac{H_{s} H_{t}}{H_{k}}-T_{\mu l}^{-1} T_{\lambda i}^{-1} T_{k n}^{-1} T_{l k} T_{i k} T_{m s} T_{n t} \frac{H_{s} H_{t}}{H_{k}}\right)
$$

where, again, summation is implied over the repeated indices and where we have used the definition $D_{i}=T_{i j} H_{j}$. Notice that equation B.11 has the expected $1 / N$ dependence. Armed with equations B.5 and B.11 we can then calculate the unfolded jet fractions and their associated errors.

\section{B.3 The Factor Method}

As the name implies, the factor method relies in multiplying the observed distributions with pre-determined factors in order to 'divide-out' the detector effects. The underlying assumption for the applicability of this scheme is that [98] the detector effects are, to a good approximation, not dominated by resolution effects. In addition, there are acceptance effects that affect the 'efficiency' of binning the distribution in question. This degradation in efficiency could be due to, for instance, clusters lost in the beampipe or discarded by an analysis cut. It also includes selection acceptance effects (good events that failed the selection cuts).

In the case of finite resolution effects (as with every real measurement) care must be taken in ensuring that the distributions to be corrected have been binned appropriately. This usually corresponds to choosing bins that are at least the same size as the resolution for the variable in question. This procedure then minimizes the bin-to-bin migration of events in a histogram.

\section{Method Outline}

Here we use the same notation as in Section B.2. Since we are now assuming that on average resolution effects can be corrected with single factors, we again use Equation B.3 but this time the matrix $T$ is a diagonal matrix. The lack of off-diagonal elements is just a reflection of the lack of correlations. We can then write the analogue of B.5:

$$
\vec{H}=C \vec{D}
$$


where now $C$ is a diagonal matrix which depends on $y$. Since there are no correlations in the matrix $C$, we can then determine its elements by looking at independent distributions of $\vec{D}$ and $\vec{H}$ from the Monte Carlo. Thus,

$$
C_{i i}^{y}=\frac{H_{i}^{y}}{D_{i}^{y}}
$$

where now the $\vec{H}$ and $\vec{D}$ distributions are independent and thus have different normalizations. In the above equation, $\vec{D}$ includes distortions due to event selection inefficiencies and resolution.

This method is also referred to as the bin-by-bin correction method. In this case, we generally don't need to worry about additional statistical errors introduced into our measurement by the use of Equation B.13. Since the Monte Carlo sample is not statistically diluted to determine a full matrix, the statistical uncertainties due to the unfolding can generally be ignored provided the sample is adequately large.

\section{B.4 Validating the Factor Method}

Due to the limited Monte Carlo sample and the fact that the inversion method suffers an additional statistical dilution, we choose to use the factor method in the final analysis. We will still carry out the analysis in parallel as a cross check for possible systematic effects.

Before going on to use the factor method we still need to show that the method is 'linear enough' to allow an unbiased measurement.

\section{A Test Experiment}

In order to test the factor method, a "test experiment" was created with a fictitious detector. The idea was to use a sample of Monte Carlo events to unfold a detectorsmeared "measurement" using an analogue of the method used in the analysis. The test measurement used was the same used in this thesis: the measurement of $\Lambda_{\overline{M S}}$ using jet-rates.

The "physics" data were generated using the LUND Monte Carlo (see Chapter 2) using the matrix element $\mathcal{O}\left(\alpha_{s}^{2}\right)$ option. The value of $\alpha_{s}$ was changed by changing the generator-level (i.e. the value used in the analytic calculations) value of $\Lambda_{\overline{M S}}$. The "detector" was simulated by using both the LUND and HERWIG Monte Carlos with the parton shower option. The parton shower $\Lambda_{Q C D}$ was varied (only for LUND) to investigate possible systematic effects. The generated particles were then smeared into a fictitious detector. This detector simulation consisted of subjecting each particle to:

- A calorimeter-like energy smearing of $\sigma / E=0.6 / \sqrt{E}+0.05$ 


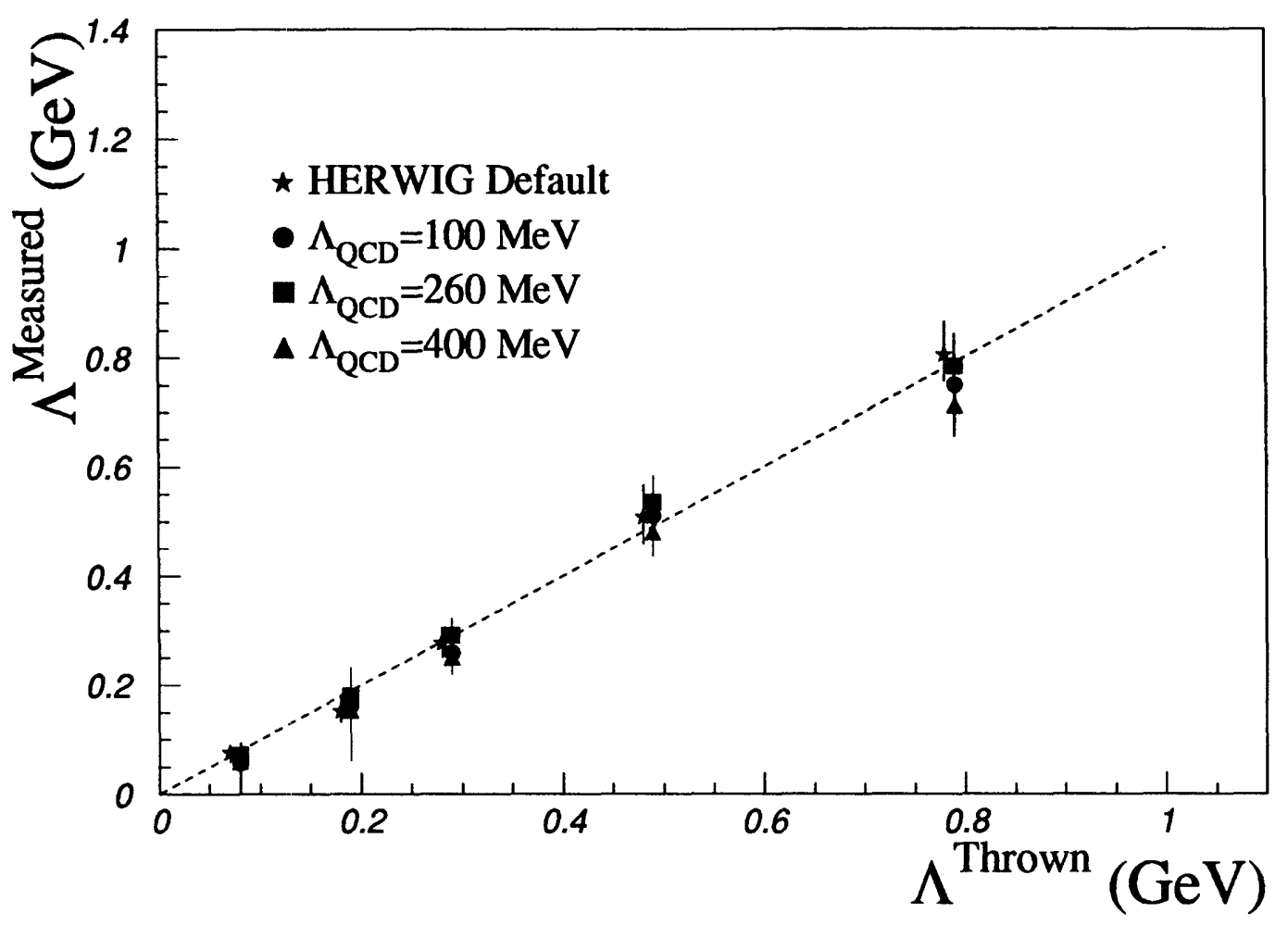

Figure B-2: $\quad \Lambda_{\overline{M S}}$ (thrown) vs $\Lambda_{\overline{M S}}$ (measured) for the Test Experiment. The star corresponds to HERWIG 5.3 correction factors, while the three other symbols correspond to $\Lambda_{\text {eff }}$ variations of the parton showers in the LUND 6.3 Monte Carlo. The dashed line represents $\Lambda^{\text {thrown }}=\Lambda^{\text {measured }}$.

- An acceptance cut of $|\cos \theta| \leq 0.95$

- A minimum energy cut of $E \geq 0.100 \mathrm{GeV}$

- Neutrinos and muons were ignored.

Finally, the factor method was applied to five different "physics" data samples generated with different values of $\Lambda_{\overline{M S}}$. The resulting $D_{2}$ distributions were fit (as in Chapter 6) to the $\mathcal{O}\left(\alpha_{s}^{2}\right)$ analytic expression (with $\mu=M_{Z^{0}}$ ) and the 'measured' $\Lambda_{\overline{M S}}$ were obtained. The results are shown in Figure B-2.

\section{Discussion}

It should be clear from Figure B-2 that the factor method provides a good correlation between the generated $\Lambda_{\overline{M S}}$ and the reconstructed $\Lambda_{\overline{M S}}$. This correlation holds for the investigated range of $\alpha_{s}$ values $\alpha_{s} \approx 0.09-0.15$. It is also clear that the method is 
somewhat sensitive to at least one of the physics parameters in the Lund generator (as expected, a small source of systematic uncertainties). In addition, systematic effects in the procedure due to different hadronization models are practically non-existent, as evidenced by the small Lund-HERWIG differences. This is consistent with our ansatz that the unfolding procedures should only 'divide-out' the detector effects.

It is then evident that the factor correction procedure provides us with an unbiased tool to correct our data sample for detector effects. 


\section{Bibliography}

[1] S. Weinberg. A model of leptons. Physical Review Letters, 19:1264, 1967.

[2] S. L. Glashow. Partial symmetries of weak interactions. Nuclear Physics, 22:579, 1961.

[3] A. Salam and J.C. Ward. Electromagnetic and weak interactions. Physics Letters, 13:168, 1964.

[4] G. Arnison et al. Experimental observation of isolated large transverse energy electrons with associated missing energy at $\sqrt{s}=540 \mathrm{GeV}$. Physics Letters, 122B(1):103, 1983.

[5] G. Altarelli. QCD and experiment: Status of $\alpha_{s}$. In P. M. Zerwas and H. A. Kastrup, editors, Proceedings, QCD: 20 Years Later, pages 172-202, 1993.

[6] R. K. Ellis and N. J. Stirling. QCD and collider physics. Fermilab-CONF-90-164-T, Fermilab, 1990.

[7] M. Gell-Mann. A schematic model of baryons and mesons. Physics Letters, 8(3):214, 1964.

[8] G. Zweig. CERN-Report 8182/TH 401, CERN, 1964.

[9] M. Y. Han and Y. Nambu. Three-triplet model with double SU(3) symmetry. Physical Review, 139(4B):1006, 1965.

[10] J. I. Friedman and H. W. Kendall. Annual Review of Nuclear Science, 22:203, 1972.

[11] H. W. Kendall. Deep inelastic scattering: Experiments on the proton and the observation of scaling. Reviews of Modern Physics, 63(3):597-614, 1991.

[12] J. I. Friedman. Deep inelastic scattering: Comparisons with the quark model. Reviews of Modern Physics, 63(3):614-627, 1991.

[13] D. J. Gross and F. Wilczeck. Ultraviolet behavior of non-Abelian gauge theories. Physical Review Letters, 30(26):1343-1346, 1973.

[14] D. H. Politzer. Reliable perturbative results for strong interactions? Physical Review Letters, 30(26):1346-1349, 1973.

[15] C. N. Yang and R. L. Mills. Conservation of isotopic spin and isotopic gauge invariance. Physical Review, 96(1):191-195, 1954.

[16] T. Muta. Foundations of Quantum Chromodynamics. World Scientific, 1987.

[17] H. Fritzsch, M. Gell-Mann, and H. Leutwyler. Advantages of the color octet gluon picture. Physics Letters, 47B(4):365-368, 1973.

[18] C. G. Callan et al. Toward a theory of the strong interactions. Physical Review D., 17(10), 1978.

[19] R. D. Field. Applications of Perturbative QCD. Addison Wesley Co., 1989.

[20] K. Nishijima. Fields and Particles. Benjamin/Cummings Publishing Co., 1969. 
[21] G. 't Hooft and M. Veltman. Nuclear Physics, B44:189, 1972.

[22] D. W. Duke and R. G. Roberts. Determination of the QCD strong coupling $\alpha_{s}$ and the scale $\Lambda_{Q C D}$. Physics Reports, 120(5):275-368, 1985.

[23] P. M. Stevenson. Comment on 'an alternative implementation of the principle of minimal sensitivity'. Physical Review D., 27:1968-1969, 1983.

[24] S. J. Brodsky et al. On the elimination of scale ambiguities in perturbative Quantum Chromodynamics. Physical Review D., 28:228, 1983.

[25] D. J. Gross and F. Wilczeck. Asymptotically free gauge theories. I. Physical Review D, 8(10):3633-3652, 1973.

[26] G. Kramer. Theory of Jets in Electron-Positron Annihilation. Springer-Varlag, 1984.

[27] M. Gell-Mann and F. E. Low. Physical Review, 95:1300, 1954.

[28] Particle Data Book. Review of particle properties. Physical Review D., 45(11), 1992.

[29] W. J. Marciano. Flavor thresholds and $\Lambda$ in the modified minimal-subtraction scheme. Physical Review D., 29(3), 1983.

[30] M. Bace. Comment on the perturbation-theoretic parameter $\Lambda$ of QCD. Physics Letters, 78B(1), 1978.

[31] J. D. Bjorken and S. J. Brodsky. Statistical model for electron-positron annihilation into hadrons. Physical Review D, 1:1416-1420, 1970.

[32] G. Hanson et al. Evidence for jet structure in hadron production by $e^{+} e^{-}$annihilation. Physical Review Letters, 35(24):1609-1612, 1975.

[33] S. L. Wu. $e^{+} e^{-}$physics at PETRA: The first 5-years. Physics Reports, 107:59, 1984.

[34] Z. Kunszt and P. Nason. QCD. In G. Altarelli et al., editor, $Z$ Physics at LEP, volume 1, pages 373-453. CERN, 1989.

[35] J. Ellis et al. Search for gluons in $e^{+} e^{-}$annihilation. Nuclear Physics, B111:253, 1976.

[36] S. Bethke. Jets in $Z^{0}$ decays. In P. M. Zerwas and H. A. Kastrup, editors, Proceedings, QCD: 20 Years Later, pages 43-71, 1993.

[37] T. D. Lee and M. Nauenberg. Physical Review, 133B:1549, 1964.

[38] S. Weinberg and G. Sterman. Jets from quantum chromodynamics. Physical Review Letters, 39:1436-1446, 1977.

[39] R. K. Ellis et al. The perturbative calculation of jet structure in $e^{+} e^{-}$annihilation. Nuclear Physics, B178:421-456, 1980.

[40] G. Kramer and B. Lampe. Two-jet cross section in $e^{+} e^{-}$annihilation. Z. Phys. C, 34:497-522, 1987.

[41] S. Bethke et al. New jet cluster algorithms: Next-to-leading order QCD and hadronization corrections. Nuclear Physics, B370:310-334, 1992.

[42] B. Bambah et al. QCD generators. In G. Altarelli et al., editor, Z Physics at LEP, volume 3, pages 143-340. CERN, 1989.

[43] D. Buskulic et al. Properties of hadronic $\mathrm{Z}$ decays and test of QCD generators. Z. Phys. C, 55:209-234, 1992.

[44] B. Adeva et al. Studies of hadronic event structure and comparisons with QCD models at the $Z^{0}$ resonance. $Z$. Phys. $C, 55: 39-61,1992$.

[45] T. Sjöstrand. The lund montecarlo for jet fragmentation and $e^{+} e^{-}$physics - JETSET 6.2 . Comp. Phys. Comm., 39:347-407, 1986. 
[46] G. Altarelli and G. Parisi. Asymptotic freedom in parton language. Nuclear Physics B, 126:298-318, 1977.

[47] T. Hansl-Kozanecka. Experimental tests of QCD: DIS, $e^{+} e^{-}$annihilation, and hadron-hadron scattering. LPNHE-92-03, U. Paris, MIT, 1992.

[48] B. Andersson. The lund model. Nuclear Physics A, 461:513-520, 1987.

[49] B. Andersson et al. A general model for jet fragmentation. Z. Phys. C, 20:317-329, 1983.

[50] G. Marchesini et al. HERWIG 5.1 - a montecarlo event generator for simulating hard emission reactions with interfering gluons. Comput. Phys. Commun., 67:465-508, 1992.

[51] W. Bartel et al. Experimental studies on multi-jet production in $e^{+} e^{-}$annihilation at PETRA energies. Z. Phys. C, 33:23, 1986.

[52] W. Braunschweig et al. Analysis of multijet final states in $e^{+} e^{-}$annihilation. Physics Letters, B214:286-294, 1988.

[53] P. N. Burrows. Private communication, 1993.

[54] M. Breidenbach. SLC and SLD - experimental experience with a linear collider. SLAC-PUB6313, SLAC, 1993.

[55] M. L. Swartz. First measurement of the left-right $\mathrm{Z}$ cross section asymmetry in polarized $e^{+} e^{-}$ collisions at the SLC. SLAC-PUB-6034, SLAC, 1993.

[56] D. Schultz et al. Polarized source performance in 1992 for SLC-SLD. SLAC-PUB-6060, SLAC, 1993.

[57] R. H. Siemann. Introduction to the physics of particle accelerators. In T. Ferbel, editor, Techniques and Concepts of High-Energy Physics VI, pages 283-323. NATO, 1990.

[58] R. H. Siemann. Electron-positron colliders and other accelerator technologies. SLAC-PUB5955, Stanford, 1993.

[59] M. J. Fero. The compton polarimeter for SLC. SLAC-PUB-6026, MIT, 1992.

[60] G. Blaylock. The WISRD beam energy measurement. SLD Physics Note 22, SLAC, 1993.

[61] SLD Collaboration. First measurement of the left-right cross section asymmetry in $\mathrm{Z}$ boson production by $e^{+} e^{-}$collisions. Physical Review Letters, 70(17):2515, 1993.

[62] SLD Collaboration. The SLD design report. SLAC report 273, SLAC, 1984.

[63] C. D. Agnew et al. XXVI international conference on high energy physics. In T. Ferbel, editor, Dallas HEP, pages 1862-1866. Dallas, 1992.

[64] S. C. Berridge. Beam test of the SLD silicon-tungsten luminosity monitor. IEEE Trans. Nucl. Sci., 37:1191-1198, 1990.

[65] K. T. Pitts. Luminosity measurement for the 1992 run. SLD Physics Note 20, U. of Oregon, 1993.

[66] D. C. Williams. Private communication, 1993.

[67] R. Wigmans. Calorimetry in high energy physics. In T. Ferbel, editor, Techniques and Concepts of High-Energy Physics VI, pages 325-379. NATO, 1990.

[68] W. J. Willis et al. Liquid-argon ionization chambers as total-absorption detectors. Nuclear Instruments and Methods, 120:221-236, 1974.

[69] U. Micke et al. $\pi / e$ discrimination with a Pb-LAr calorimeter. Nuclear Instruments and Methods, 221:495-502, 1984.

[70] E. J. Wicklund. Inclusive Electron Production from Heavy Quarks with the TASSO Pb-LAr Calorimeters in $e^{+} e^{-}$Annihilation at PETRA. Phd Thesis, Wisconsin, 1984. 
[71] P. Schacht. Calibration of the $\mathrm{H} 1$ liquid argon calorimeter. Nuclear Instruments and Methods, A327:212-215, 1993.

[72] W. Braunschweig et al. Results from a test of a Pb-Fe liquid argon calorimeter. Preprint 89-022, DESY, 1989.

[73] R. Wigmans. On the energy resolution of uranium and other hadron calorimeters. Nuclear Instruments and Methods, A259:389, 1987.

[74] G. S. Abrams et al. Performance of the Pb/LAr shower counter system of the MARK II detector at SPEAR. IEEE Trans. on Nucl. Sci., NS-27(1):59-63, 1980.

[75] $\mathrm{D}$. Axen et al. The $\mathrm{Pb}-\mathrm{Ar}$ sampling calorimeter of the SLD detector. Nuclear Instruments and Methods, A328:472-494, 1993.

[76] E. N. Vella. SLD liquid argon calorimeter. SLAC-PUB 5953, SLD, 1992.

[77] S. Jadach et al. The monte carlo program KORALZ, version 3.8, for the lepton or quark pair production at LEP/SLC energies. Comput. Phys. Commun., 66:276-292, 1991.

[78] B.F.L. Ward et al. Montecarlo BHLUMI-2.01 for Bhabha scattering at low angles with YennieFrautschi-Suura exponentiation. Comp. Phys. Comm., 70:305-344, 1992.

[79] H. Aihara et al. Measurement of the photon structure function $F_{2}^{\gamma}\left(x, Q^{2}\right)$ in the region $0.2-$ $\mathrm{GeV}^{2}<Q^{2}<7-\mathrm{GeV}^{2}$. Z. Phys., C34:1, 1987.

[80] R. Brun et al. GEANT3 user's guide. CERN-DD/EE/84-1, CERN, 1989.

[81] G. Grindhammer et al. The fast simulation of electromagnetic and hadronic showers. Nuclear Instruments and Methods, A290:469, 1990.

[82] E. N. Vella. LAC charge collection, sampling, gain, and digitization. LAC Software Note 89-2, SLD, 1989.

[83] E. Bernardi et al. Performance of a compensating lead-scintillator hadronic calorimeter. $\mathrm{Nu}$ clear Instruments and Methods, A262:229, 1987.

[84] J.M. Yamartino. A Measurement of the $e^{+} e^{-}$Decay Width of the $Z^{0}$. Phd Thesis, MIT, 1994.

[85] W. B. Atwood. Private communication, 1992.

[86] T. Åkesson et al. Performance of the U/plastic scintillator calorimeter for the Helios experiment at CERN. Nuclear Instruments and Methods, A262:243, 1987.

[87] A. G. Frodesen et al. Probability and Statistics in Particle Physics. Universitetsforlaget, 1979.

[88] G. Feldman. Muon backgrounds at the SLC. Mark II internal memo., SLAC, 1988.

[89] S. González. Report on muon background montecarlo study. SLD Note 214, SLD, 1992.

[90] E. N. Vella et al. Tower thresholds for LAC total energy trigger. SLD Note 213, SLD, 1992.

[91] D. Decamp et al. Measurement of the charged particle multiplicity distribution in hadronic $\mathrm{Z}$ decays. Physics Letters, B273:181-192, 1991.

[92] B. Adeva et al. Measurement of the inclusive production of neutral pions and charged particles on the $Z^{0}$ resonance. Physics Letters, B259:199-208, 1991.

[93] K. T. Pitts. Private communication, 1993.

[94] R. Ben-David and H. Park. Background measurement for the $1992 A_{L R}$ analysis. SLD Physics Note 18, SLAC, 1993.

[95] P. N. Burrows et al. Estimate of combined triggering and selection efficiency for hadronic events. SLD Note 229, SLAC, 1993. 
[96] S. Catani et al. New clustering algorithm for multijet cross sections in $e^{+} e^{-}$annihilation. Physics Letters, B269:432-438, 1991.

[97] M. Z. Akrawy et al. A study of jet production rates and a test of QCD on the $Z^{0}$ resonance. Physics Letters, B235:389, 1990.

[98] V. Blobel. Unfolding methods in high-energy physics experiments. In 1984 CERN School of Computing, pages 88-127. CERN, 1984.

[99] F. James and M. Roos. MINUIT manual. CERN-D506, CERN, 1989.

[100] P. D. Acton et al. A global determination of $\alpha_{s}\left(M_{Z^{\circ}}\right)$ at LEP. Z. Phys. C, 55:1-24, 1992.

[101] D. H. Politzer. Stevenson's optimized perturbation theory applied to factorization and mass scheme dependence. Nuclear Physics B, 194:493-512, 1982.

[102] S. Bethke et al. Experimental investigation of the energy dependence of the strong coupling strength. Physics Letters, B213:235-241, 1988.

[103] K. Abe et al. Determination of the QCD scale parameter $\Lambda_{\overline{M S}}$ with QCD cascade on the basis of the next-to-leading logarithmic approximation. Physics Letters, B240:232-236, 1990.

[104] I. H. Park et al. Experimental evidence for the non-Abelian nature of QCD from a study of multijet events produced in $e^{+} e^{-}$annihilations. Physical Review Letters, B248:1713-1716, 1989.

[105] B. Adeva et al. Determination of $\alpha$, from jet multiplicities measured on the $Z^{0}$ resonance. Physics Letters, B248:464-472, 1990.

[106] M. Z. Akrawy et al. A study of the recombination scheme dependence of jet production rates and of $\alpha_{s}\left(M_{Z}\right)$ in hadronic $Z^{0}$ decays. Z. Phys. C, 49:375-384, 1991.

[107] S. Bethke et al. Studies of jet production rates in $e^{+} e^{-}$annihilation at $E_{c m}=29 \mathrm{GeV} . Z$. Phys. C, 43:325-330, 1989.

[108] F. Wilczek. QCD and asymptotic freedom: Perspectives and prospects. In P. M. Zerwas and H. A. Kastrup, editors, Proceedings, QCD: 20 Years Later, pages 17-38, 1993.

[109] D. Buskulic et al. Measurement of the strong coupling constant using $\tau$ decays. Physics Letters, B307:209-220, 1993.

[110] J. Alitti et al. Physics Letters, B263:563, 1991.

[111] S. Komamiya et al. Determination of $\alpha_{s}$ from a differential-jet-multiplicity distribution in $e^{+} e^{-}$collisions at $\sqrt{s}=29 \mathrm{GeV}$ and $91 \mathrm{GeV}$. Physical Review Letters, 64(9):987-990, 1990.

[112] K. Abe et al. A measurement of $\alpha_{s}$ from jet rates at the $Z^{0}$ resonance. Physical Review Letters, 71:2528-2532, 1993.

[113] S. Bethke and S. Catani. A summary of $\alpha_{s}$ measurements. CERN-TH-6484/92, CERN, 1992.

[114] D. Decamp et al. Evidence for the triple gluon vertex from measurements of the QCD color factors in Z decay into four jets. Physics Letters, B284:151-162, 1992.

[115] E. Farhi. Quantum chromodynamics test for jets. Physical Review Letters, 39(25):1587, 1977.

[116] L. Rosenson. Private communication, 1993. 